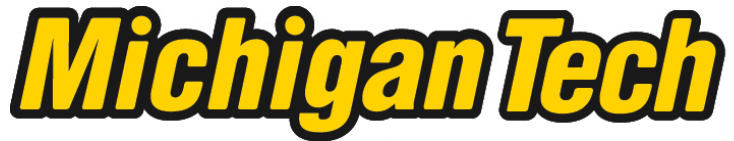 \\ Michigan Technological University Create the Future Digital Commons @ Michigan Tech
}

2015

SYNTHETIC OLIGODEOXYNUCLEOTIDE PURIFICATION VIA CATCHING BY POLYMERIZATION

Suntara Fueangfung

Michigan Technological University

Follow this and additional works at: https://digitalcommons.mtu.edu/etds

Part of the Chemistry Commons

Copyright 2015 Suntara Fueangfung

\section{Recommended Citation}

Fueangfung, Suntara, "SYNTHETIC OLIGODEOXYNUCLEOTIDE PURIFICATION VIA CATCHING BY POLYMERIZATION", Master's Thesis, Michigan Technological University, 2015.

https://doi.org/10.37099/mtu.dc.etds/900

Follow this and additional works at: https://digitalcommons.mtu.edu/etds

Part of the Chemistry Commons 


\title{
SYNTHETIC OLIGODEOXYNUCLEOTIDE PURIFICATION VIA CATCHING BY POLYMERIZATION
}

By

Suntara Fueangfung

\begin{abstract}
A DISSERTATION
Submitted in partial fulfillment of the requirements for the degree of

DOCTOR OF PHILOSOPHY

In Chemistry
\end{abstract}

MICHIGAN TECHNOLOGICAL UNIVERSITY

2015

(C) 2015 Suntara Fueangfung 
This dissertation has been approved in partial fulfillment of the requirements for the Degree of DOCTOR OF PHILOSOPHY in Chemistry.

Department of Chemistry

Dissertation Advisor: Dr. Shiyue Fang

Committee Member: Dr. Haiying Liu

Committee Member: Dr. Lanrong Bi

Committee Member: Dr. Claudio Mazzoleni

Department Chair: Dr. Cary Chabalowski 
To my parents and my sister 


\section{Table of Contents}

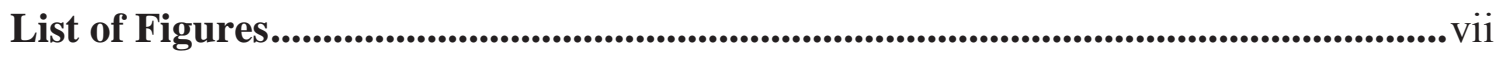

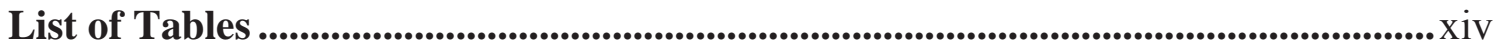

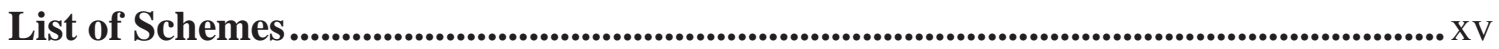

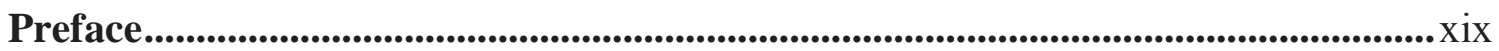

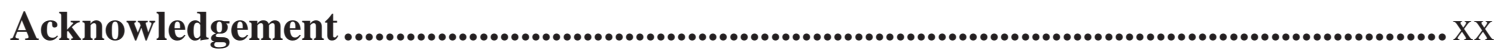

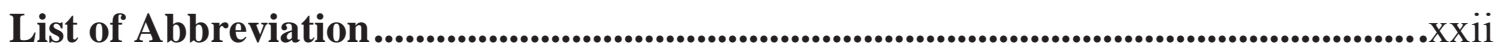

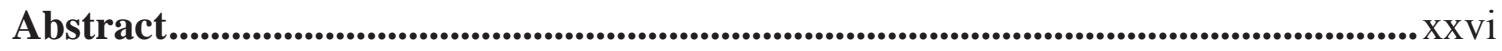

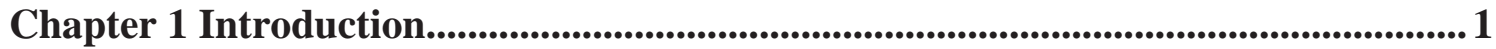

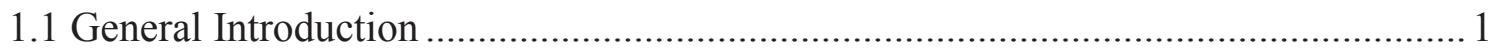

1.2 The New ODN Purification Technology ............................................................ 2

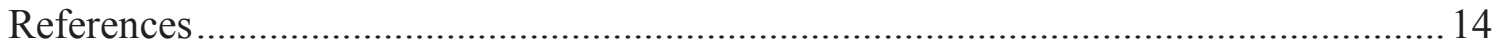

Chapter 2 History and Background ......................................................................... 16

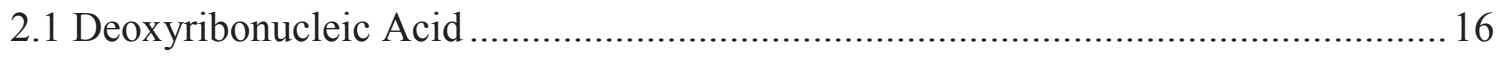

2.2 Oligodeoxynucleotide and Applications ....................................................... 21

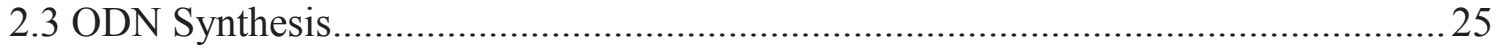

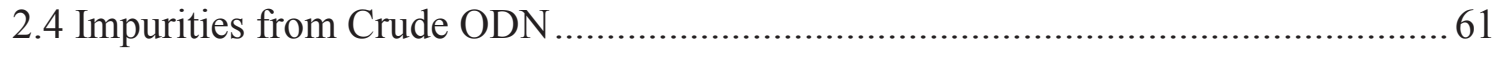

2.5 Current Methods for ODN Purification and Limitations ....................................... 64 


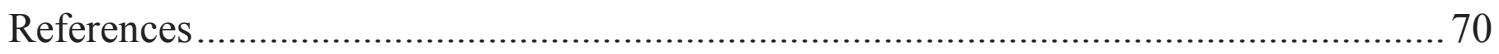

\section{Chapter 3 Scalable Synthetic Oligodeoxynucleotide Purification with Use of a}

Catching by Polymerization, Washing, and Releasing Approach............................... 76

Abstract

3.1 Introduction 78

3.2 Results and Discussion 78

3.3 Conclusions 87

3.4 Experimental Section 87

Acknowledgements. 106

References and Notes 107

Chapter 4 Synthetic Oligodeoxynucleotide Purification by Polymerization of Failure

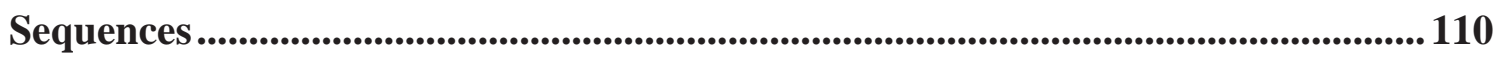

Abstract

4.1 Introduction

4.2 Results and Discussion

4.3 Conclusions 
Acknowledgements

References and Notes

Chapter 5 Synthetic 5'-Phosphorylated Oligodeoxynucleotide Purification through

Catching Full-length Sequences by Polymerization

Abstract

5.1 Introduction

5.2 Results and Discussion

5.3 Conclusions

5.4 Experimental Section

Acknowledgements

References

Chapter 6 Future Research Plan

6.1 User-friendly Cleavable Linker for ODN Purification by Catching Full-length

Sequence 162

6.2 Detailed Studies on ODN Purification by Capping Failure Sequences . 165

6.3 Synthetic Peptide Purification using Polymerization Approach. 166

Appendix A. Supporting Information for Chapter 3 168

Appendix B. Supporting Information for Chapter 4. 
Appendix C. Supporting Information for Chapter 5

Appendix D. Permission from Publishers................................................................................ 203

\section{List of Figures}

\section{Chapter 1 Introduction}

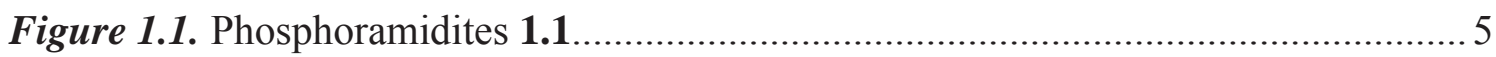

Figure 1.2. Phosphoramidites 1.2

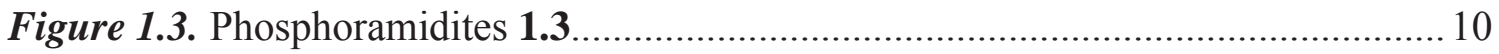

\section{Chapter 2 History and Background}

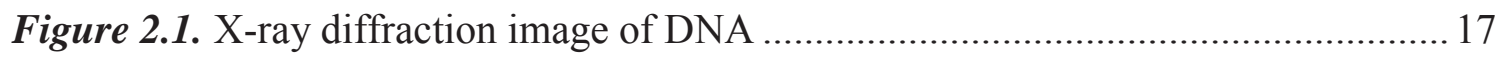

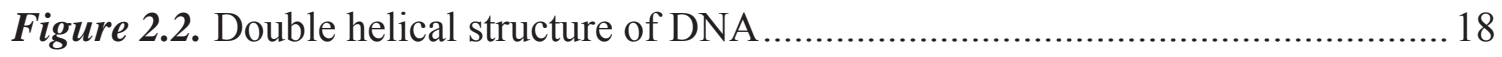

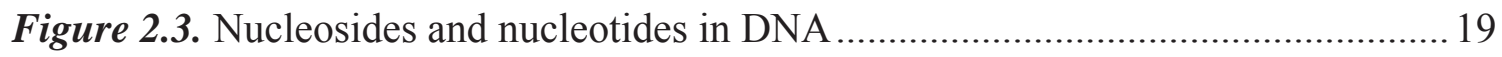

Figure 2.4. Hydrogen bonding for each base pair in double-stranded DNA ................. 20

Figure 2.5. (1) structure of Fomivirsen (5'-GCG TTT GCT CTT CTTCTT GCG-3'), (2) structure of Mipomersen (5'-G*-C*-C*-U*-C*-AGTCTG-d(5-methyl-C)-TT-d(5-

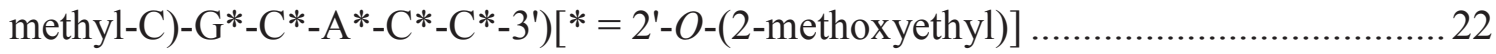


Figure 2.6. The new version of solid support attaching to 3 '-end of the initial nucleoside

Figure 2.7. Deoxynucleoside phosphoramidites 46

Figure 2.8. Pac-dA-CE phosphoramidite and $i$ Pr-Pac-dG-CE phosphoramidte for Ultramild ODN synthesis. 46

Figure 2.9. Example of deletion sequence (right). The $\mathrm{dG}$ at $8^{\text {th }}$ position was missing compared to the full-length sequence (left)

\section{Chapter 3 Scalable Synthetic Oligodeoxynucleotide Purification with Use of a} Catching by Polymerization, Washing, and Releasing Approach

Figure 3.1. RP HPLC profile of crude ODN.

Figure 3.2. RP HPLC profile of impurities including 3.7

Figure 3.3. RP HPLC profile of ODN 3.9 purified by polymerization, washing, and releasing approach

Figure 3.4. RP HPLC profiles: (a) co-injection of ODN 3.9 and control ODN; (b) control ODN .85

Figure 3.5. HPLC profile of recovered guanosine. .98

Figure 3.6. HPLC profile of authentic guanosine .98

Figure 3.7. HPLC profile of authentic 8-oxo-guanosine .99 
Figure 3.8. Co-injection HPLC profile of recovered guanosine and authentic guanosine

Figure 3.9. Co-injection HPLC profile of recovered guanosine and authentic 8-oxoguanosine 100

Figure 3.10. HPLC profile of recovered adenosine 101

Figure 3.11. HPLC profile of authentic adenosine 101

Figure 3.12. Co-injection HPLC profile of recovered adenosine and authentic adenosine

Figure 3.13. HPLC profile of recovered thymidine 102

Figure 3.14. HPLC profile of recovered cytidine 103

Figure 3.15. HPLC profile of authentic thymidine 103

Figure 3.16. HPLC profile of authentic cytidine 104

Figure 3.17. Co-injection HPLC profile of recovered thymidine and authentic thymidine 104

Figure 3.18. Co-injection HPLC profile of recovered cytidine and authentic cytidine ...... 105 
Chapter 4 Synthetic Oligodeoxynucleotide Purification by Polymerization of Failure Sequences

Figure 4.1. Structure of capping phosphoramidite 4.1................................................ 114

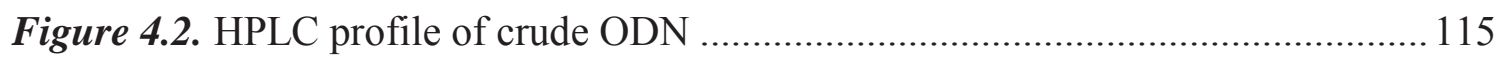

Figure 4.3. HPLC profile of pure ODN after polymerization....................................... 117

Figure 4.4. RP HPLC profiles of pure ODN 4.4 after (a) polymerization and desalting,

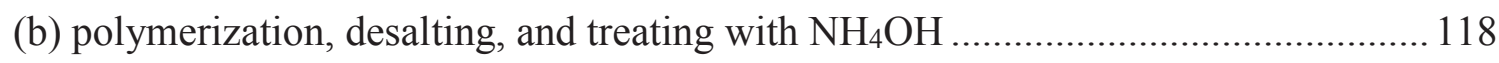

Figure 4.5. RP HPLC profiles of (a) co-injection of ODN 4.4 with control, (b) control

ODN

Figure 4.6. $\mathrm{RP}$ HPLC profiles of $\mathrm{ODN} 4.4$ after polymerization, $\mathrm{NH}_{4} \mathrm{OH}$, and $n-\mathrm{BuOH}$

precipitation

Chapter 5 Synthetic 5'-Phosphorylated Oligodeoxynucleotide Purification through Catching Full-length Sequences by Polymerization

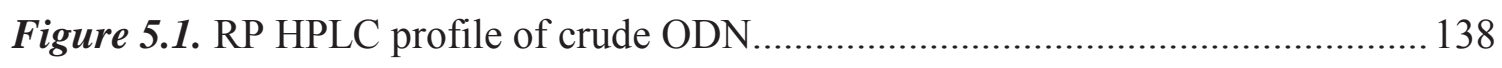

Figure 5.2. RP HPLC profile of impurities including failure sequences 5.5 ............... 142

Figure 5.3. RP HPLC profile of purified ODN 5.8 …………………….................. 142

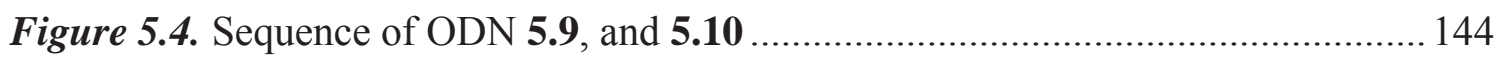


Figure 5.5. RP HPLC profiles: (a) crude 61-mer ODN 5.9; (b) purified 61-mer ODN 5.10

Figure 5.6. Sequence of ODN 5.11, and $\mathbf{5 . 1 2}$ 146

Figure 5.7. RP HPLC profiles: (a) crude 25-mer ODN 5.11; (b) purified 25-mer ODN

5.12 . 147

\section{Chapter 6 Future Research Plan}

Figure 6.1. Phosphoramidite 6.1 163

Figure 6.2. New polymerizable capping phosphoramidites. 165

Figure 6.3. Compound 6.9 167

Figure 6.4. Compound 6.10 167

\section{Appendix A. Supporting Information for Chapter 3}

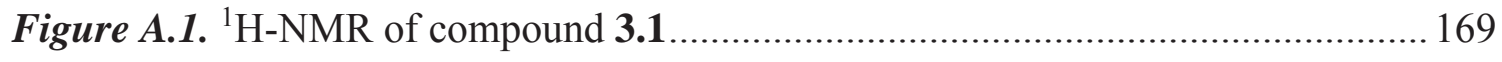

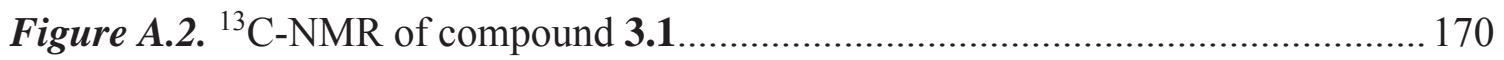

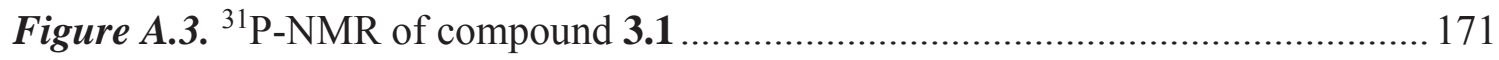

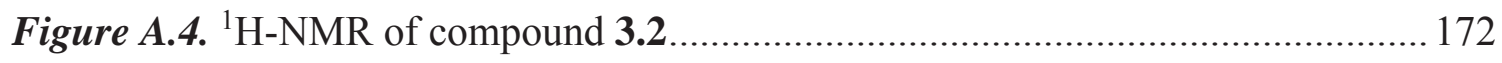

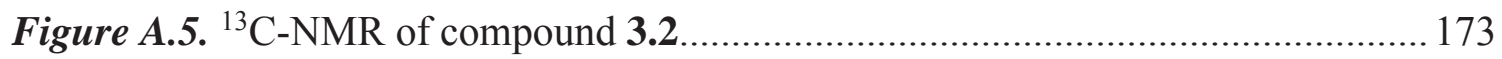


Figure A.6. ${ }^{1} \mathrm{H}-\mathrm{NMR}$ of compound 3.3

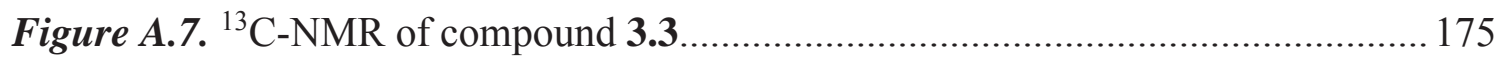

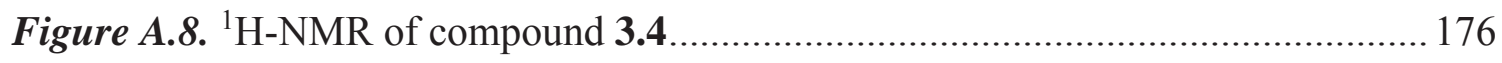

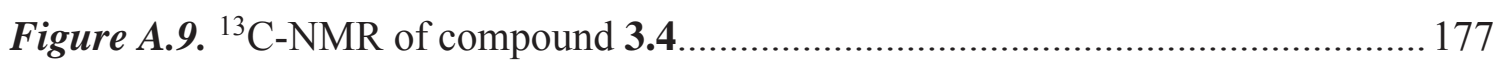

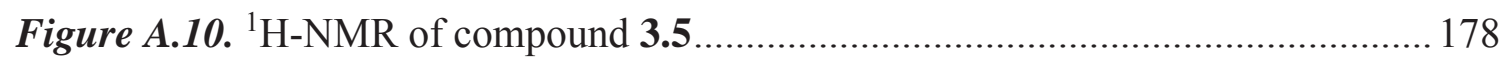

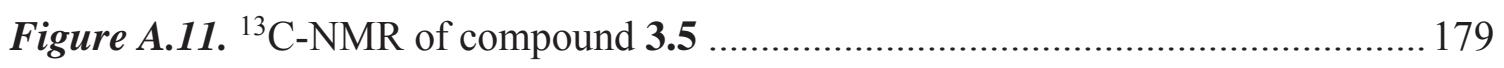

Figure A.12. MALDI-TOF mass spectrum of ODN 3.9 purified using the catching by

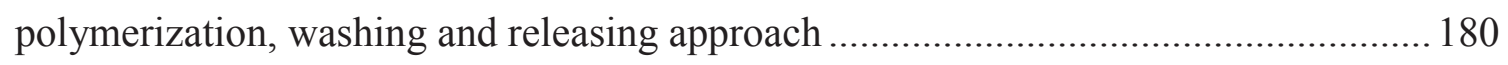

\section{Appendix B. Supporting Information for Chapter 4}

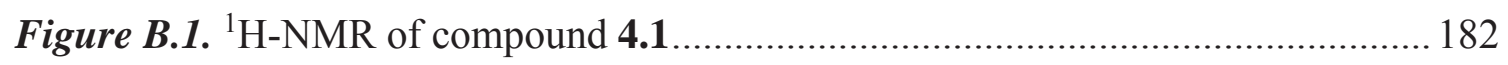

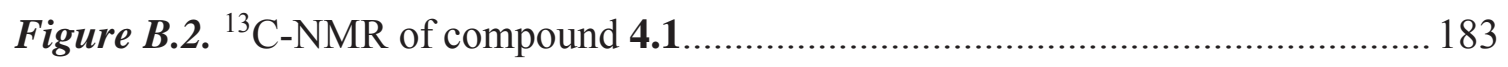

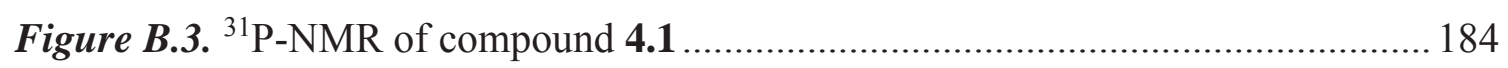

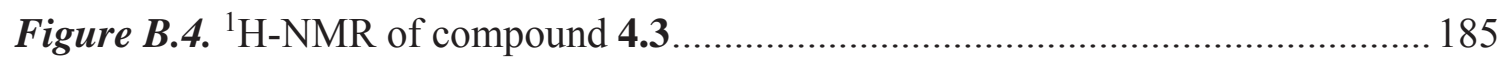

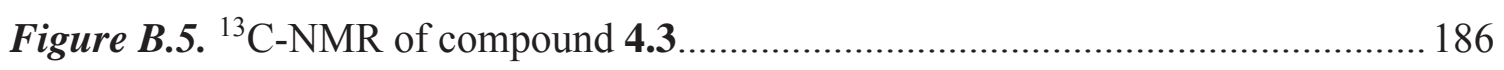

Figure B.6. MALDI-TOF mass spectrum of ODN 4.4 purified by polymerization of

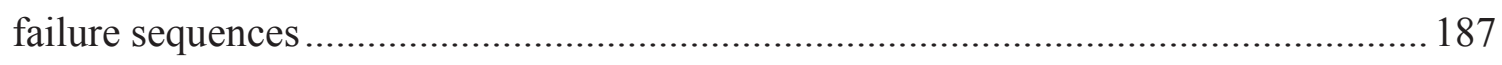

Figure B.7. HPLC profile of crude ODN with two times capping instead of four times

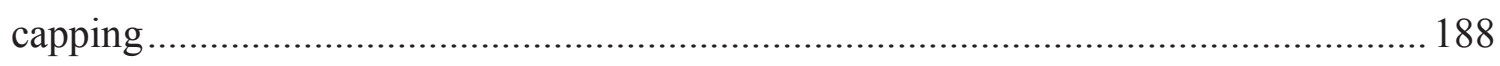




\section{Appendix C. Supporting Information for Chapter 5}

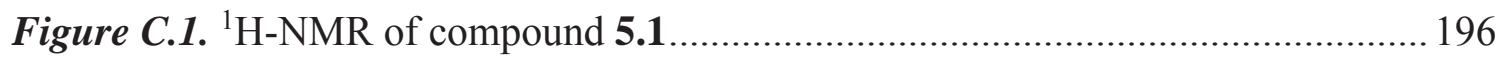

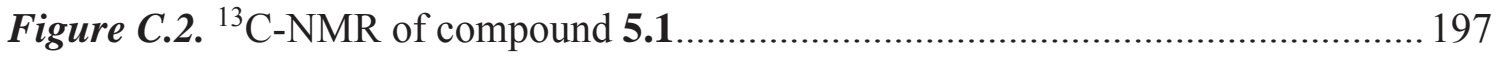

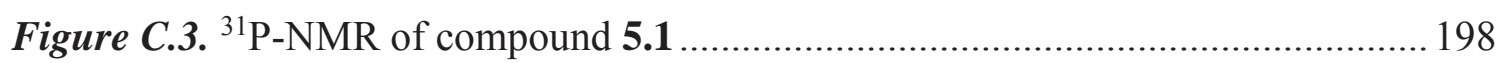

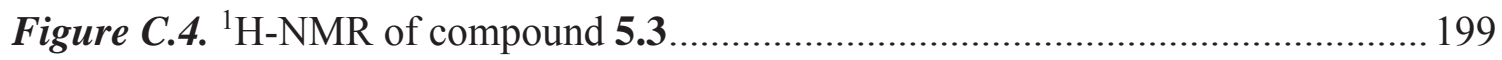

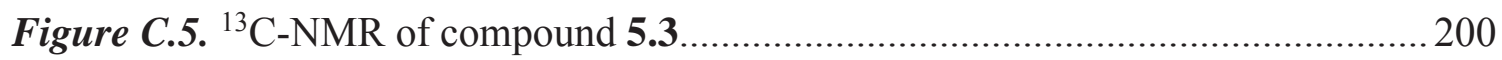

Figure C.6. MALDI-TOF mass spectrum of ODN 5.8 purified by polymerization of

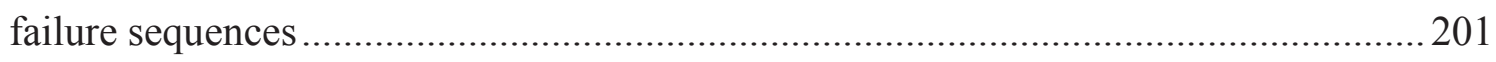

Figure C.7. MALDI-TOF mass spectrum of ODN 5.8 (Expanded) purified by

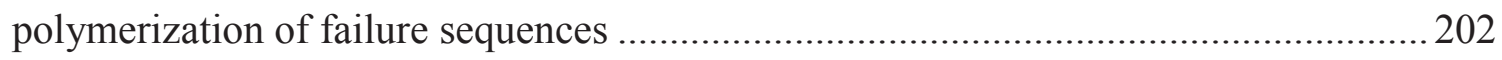




\section{List of Tables}

\section{Chapter 2 History and Background}

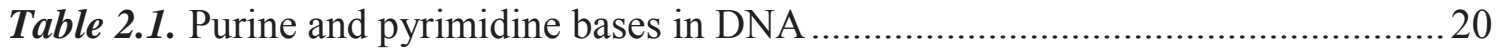

\section{Appendix B. Supporting Information for Chapter 4}

Table B.1. ODN synthetic cycle using polymerizable phosphoramidite 4.1 as capping

agent 


\section{List of Schemes}

\section{Chapter 1 Introduction}

Scheme 1.1. General design of the first method using catching full-length ODN by

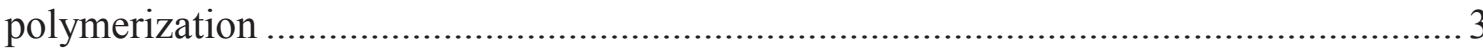

Scheme 1.2. General design of the second method using catching failure sequences by

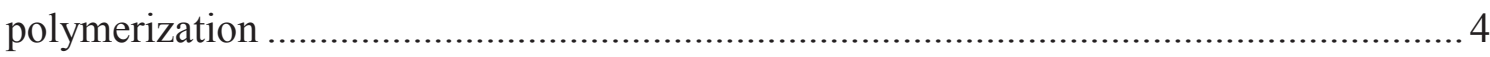

Scheme 1.3. ODN synthesis for catching full-length ODN ...................................... 6

Scheme 1.4. Catching full-length ODN by polymerization ...................................... 7

Scheme 1.5. Catching failure sequences by polymerization ..................................... 9

Scheme 1.6. 5'-Phosphorylated ODN synthesis for catching full-length ODN ............. 11

Scheme 1.7. Catching full-length5'-phosphorylated ODN by polymerization ............... 12

\section{Chapter 2 History and Background}

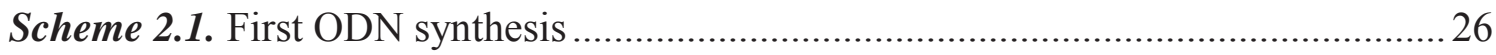

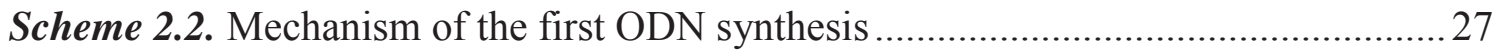

Scheme 2.3. ODN synthesis using phosphodiester method .......................................28

Scheme 2.4. Mechanism of ODN synthesis using phosphodiester method ...................29

Scheme 2.5. Branched ODN from phosphodiester synthesis ...................................... 30 
Scheme 2.6. Preparation of solid support using 5'-DMTr-protected dC...........

Scheme 2.7. ODN synthesis using phosphotriester method

Scheme 2.8. Mechanism of ODN synthesis using phosphotriester method. 35

Scheme 2.9. ODN synthesis using $H$-phosphonate method 36

Scheme 2.10. Mechanism of ODN synthesis using $H$-phosphonate method 38

Scheme 2.10 (Continued). Mechanism of ODN synthesis using $H$-phosphonate method

Scheme 2.11. Phosphonate acylation (Side reaction) of $H$-phosphonate compound .40

Scheme 2.12. Double acylation (Side reaction) of $H$-phosphonate group 40

Scheme 2.13. ODN synthesis using phosphite-triester approach 41

Scheme 2.14. Mechanism of phosphite-triester approach

Scheme 2.15. ODN synthesis using phosphoramidite approach 47

Scheme 2.16. Mechanism of detritylation .48

Scheme 2.17. Resonance structures of DMTr cation .49

Scheme 2.18. Mechanism of phosphoramidite activation and coupling . 50

Scheme 2.19. Mechanism of capping 52

Scheme 2.20. Mechanism of oxidation 54 
Scheme 2.21. Mechanism of cleavage, phosphate deprotection, and nucleobase

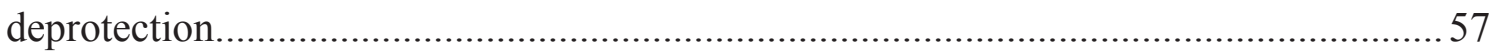

Scheme 2.22. Michael addition of adenine, cytidine, and guanine with acrylonitrile..... 58

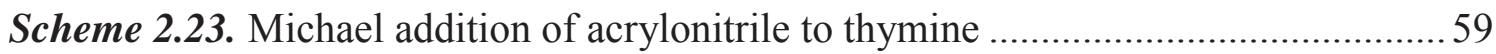

Scheme 2.24. Reversed Michael reaction in excess of ammonia ...................................60

Scheme 2.25. Mechanism of scavenging acrylonitrile in ammonia ............................60

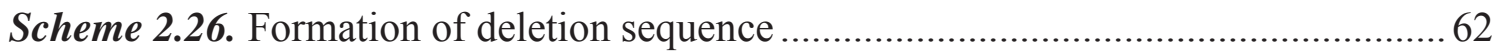

Scheme 2.27. Formation of addition sequence during coupling step ...........................63

\section{Chapter 3 Scalable Synthetic Oligodeoxynucleotide Purification with Use of a}

\section{Catching by Polymerization, Washing, and Releasing Approach}

Scheme 3.1. Synthesis of phosphoramidite 3.1 80

Scheme 3.2. Purification of ODN with use of the catching by polymerization, washing, and releasing approach

Chapter 4 Synthetic Oligodeoxynucleotide Purification by Polymerization of Failure Sequences

Scheme 4.1. Purification of ODN by polymerization of failure sequences. 116 
Chapter 5 Synthetic 5'-Phosphorylated Oligodeoxynucleotide Purification through Catching Full-length Sequences by Polymerization

Scheme 5.1. Synthesis of acrylation and phosphinylation phosphoramidite 5.1........... 137

Scheme 5.2. Purification of 5'-phosphorylated ODN through catching full-length

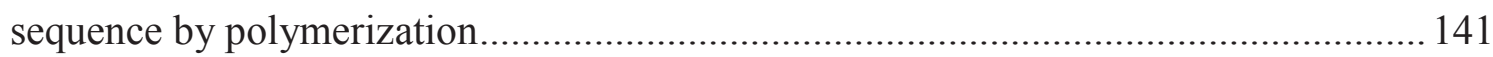

Chapter 6 Future Research Plan

Scheme 6.1. Proposed ODN purification using phosphoramidite 6.1 ........................... 164 


\section{Preface}

All contents of Chapter 1, Chapter 2, and Chapter 6 were written by Mr. Suntara Fueangfung and revised by Dr. Shiyue Fang.

All of research work in Chapter 3 was conducted and written by Mr. Suntara Fueangfung, with the exceptions of (i) testing the stability of nucleobases under radical acrylamide polymerization, which was performed by Dr. Shiyue Fang; (ii) catching fulllength ODN by polymerization was performed with the assistance of Dr. Shiyue Fang.

All of research work in Chapter 4 was conducted and written by Mr. Suntara Fueangfung, with the exceptions of (i) catching failure sequences by polymerization was performed with the assistance of Dr. Shiyue Fang; (ii) the synthesis of capping agent was performed with the assistance of Ms. Xi Lin. There are contributions of the other authors; Mr. Xiang Zhang helped maintaining instrument (HPLC) which is important for the analysis of the project; Dr. Wenpeng Mai did some early studies of this project; Dr. Lanrong Bi and Dr. Sarah A. Green helped writing proposal to purchase DNA/RNA synthesizer. Without the synthesizer, the project would not succeed.

All of research work in Chapter 5 was conducted and written by Mr. Suntara Fueangfung, with the exceptions of (i) catching full-length 5'-phosphorylated ODN by polymerization was performed by Dr. Yinan Yuan; (ii) the synthesis of compound (particularly first step of the synthesis) was assisted by Ms. Xi Lin; (iii) the preparation of the crude ODN samples prior to polymerization experiments was assisted by Mr. Durga Pokharel. All of research work was supervised by Dr. Shiyue Fang. 


\section{Acknowledgements}

I am tremendously thankful for everything which is invaluable for my $\mathrm{PhD}$ journey. First of all, I would like to express my greatest gratitude to my advisor and mentor Dr. Shiyue Fang for his support and encouragement through my PhD study. With patience, he has spent his valuable time not only giving valuable advices for my research, but also training me to be a competent and enthusiastic researcher. I have learned so much from him. His supervision is remarkably appreciated.

I would like to express my gratitude to the members of my dissertation committee: Dr. Haiying Liu, Dr. Lanrong Bi, and Dr. Claudio Mazzoleni for their time, support, and suggestion on my $\mathrm{PhD}$ dissertation and defense.

I would like thank deeply to my former and present group members: Dr. Yinan Yuan, Dr. Xiang Zhang, Dr. Zezhou Wang, Dr. Xi (Sissi) Lin, Dr. Mingcui Zhang, Dr. Bin Cao, Dr. Deepti Bansal, Durga Pokharel, Ashok Khanal, Shahien (Shawn) Shahsavari, Christopher Thomas, Nathanael Green, Lucia Li, and John Hausman. I am very appreciative for their help and supports. I wish them success in their career.

The grateful help from all staff in Department of Chemistry which includes Celine Grace, Denise Laux, Chalene Page, Magaret Dunstan, Dean Seppala, Don Wareham, Shane Crist, Jerry Lutz, and Lois Blau is greatly appreciated. I also would like to express my special thanks to Andrew Galerneau, Lorri Reilly, and Aparna Pandey who gave me the opportunity to fulfill my teaching experiences at Michigan Tech. 
I would like to express my gratitude to Dr. Pichai Sripaipan, MD and Dr. Rajanee Sripaipan, MD who have been helping me everything during my living in Houghton. I also would like to thank all Thai friends at Michigan Tech for their cheerfulness and unforgettable friendship.

I would like to thank my Michigan Tech friends: Dr. Nazmiye Yapici, Sasha Teymorian, Dr. Ee Lim Tan, Dr. Giri Vegesna, Dr. Natee Tangtrakarn, Dr. Madhana Sunder, Andrew Chapp, Srinivas Mandalapu, Jingtuo Zhang, Lawrence Wall, Nancy Byers Sprague, Dianne Sprague, and fellow graduate students in the department of Chemistry. They have supported me during my study. They continuously inspire me to have positive attitude and be a happy person.

I also would like to acknowledge The Royal Thai Government who gave me this great opportunity to study at Michigan Tech. This is one of the most wonderful experience in my life and I will never forget.

Last of all, I would like to thank my parents and my sister for their love, help, and unconditional supports throughout the years. Their encouragement has motivated me to have been through the hard time in my $\mathrm{PhD}$ study. I always love them and this dissertation is partly devoted to them. 


\section{List of Abbreviations}

$\AA$

Ac

ATRP

$\mathrm{Bz}$

CE

CPG

CSO

DBU

DCC

DCI

DIEA

DMF

$\mathrm{DMTr}$

DNA

dA

dATP
Angstrom

acetyl

atom transfer radical polymerization

benzoyl

2-cyanoethyl

controlled pore glass

(1S)-(+)-(10-camphorsulfonyl)-

oxaziridine

1,8-diazabicyclo[5.4.0]undec-7-ene

dicyclohexylcarbodiimide

4,5-dicyanoimidazole

$N, N$-diisopropylethylamine

$N, N$-dimethylformamide

4,4'-dimethoxytrityl

deoxyribonucleic acid

deoxyadenosine

deoxyadenosine triphosphate 


\begin{tabular}{|c|c|}
\hline $\mathrm{dC}$ & deoxycytidine \\
\hline $\mathrm{dCTP}$ & deoxycytidine triphosphate \\
\hline $\mathrm{dG}$ & deoxyguanosine \\
\hline dGTP & deoxyguanosine triphosphate \\
\hline dNTP & deoxynucleoside triphosphate \\
\hline $\mathrm{dT}$ & deoxythymidine \\
\hline dTTP & deoxythymidine triphosphate \\
\hline ddATP & dideoxyadenosine triphosphates \\
\hline ddCTP & dideoxycytidine triphosphates \\
\hline ddGTP & dideoxyguanosine triphosphates \\
\hline ddNTP & dideoxynucleoside triphosphates \\
\hline ddTTP & dideoxythymidine triphosphates \\
\hline eq & equivalent \\
\hline ESI & Electrospray Ionization \\
\hline FDA & Food and Drug Administration \\
\hline $\mathrm{g}$ & gram \\
\hline GC & Gas Chromatography \\
\hline $\mathrm{h}$ & hour(s) \\
\hline
\end{tabular}


HPLC

HRMS

$\mathrm{Hz}$

$i$-Bu

$i-\operatorname{Pr}$

IX

$J$

$\mathrm{K}_{\mathrm{a}}$

lcaa

[M]

M

MALDI

Min

$\mathrm{MHz}$

$\mathrm{mg}$

$\mathrm{mL}$
High Performance Liquid

Chromatography

High Resolution Mass Spectrometry

Hertz

isobutyl

isopropyl

ion-exchange

coupling constant

acid dissociation constant

long chain alkyl amino

Molecular mass

molar

Matrix-Assisted Laser

Desorption/Ionization

minute(s)

megahertz

milligram

milliliter 


\begin{tabular}{|c|c|}
\hline $\mathrm{mM}$ & millimolar \\
\hline mmol & millimole \\
\hline mRNA & messenger ribonucleic acid \\
\hline MS & Mass spectrometry \\
\hline MSNT & mesitylsulfonyl nitrotriazole \\
\hline MWCO & molecular weight cut-off \\
\hline$n-\mathrm{Bu}$ & butyl \\
\hline NMI & $N$-methylimidazole \\
\hline nmol & nanomole \\
\hline NMR & Nuclear Magnetic Resonance \\
\hline $\mathrm{ODN}(\mathrm{s})$ & Oligodeoxynucleotide(s) \\
\hline $\mathrm{ON}(\mathrm{s})$ & Oligonucleotide(s) \\
\hline$P$ & portion \\
\hline $\mathrm{Pac}$ & phenoxyacetyl \\
\hline PAGE & Polyacrylamide Gel Electrophoresis \\
\hline PCR & Polymerase Chain Reaction \\
\hline ppm & part per million \\
\hline RNA & ribonucleic acid \\
\hline
\end{tabular}




$\begin{array}{ll}\text { RP } & \text { reversed phase } \\ \mathrm{S}_{\mathrm{N}} & \text { bimolecular nucleophilic substitution } \\ \text { THF } & \text { tetrahydrofuran } \\ \text { TLC } & \text { thin layer chromatography } \\ \text { TMEDA } & N, N, N^{\prime}, N^{\prime}- \\ & \text { tetramethylethylenediamine } \\ \text { TOF } & \text { Time of flight } \\ \text { tRNA } & \text { transfer ribonucleic acid } \\ \mu \mathrm{L} & \text { microliter } \\ \mu \mathrm{mol} & \text { micromole }\end{array}$




\begin{abstract}
Large quantities of pure synthetic oligodeoxynucleotides (ODNs) are important for preclinical research, drug development, and biological studies. These ODNs are synthesized on an automated synthesizer. It is inevitable that the crude ODN product contains failure sequences which are not easily removed because they have the same properties as the full length ODNs. Current ODN purification methods such as polyacrylamide gel electrophoresis (PAGE), reversed-phase high performance liquid chromatography (RP HPLC), anion exchange HPLC, and affinity purification can remove those impurities. However, they are not suitable for large scale purification due to the expensive aspects associated with instrumentation, solvent demand, and high labor costs.
\end{abstract}

To solve these problems, two non-chromatographic ODN purification methods have been developed. In the first method, the full-length ODN was tagged with the phosphoramidite containing a methacrylamide group and a cleavable linker while the failure sequences were not. The full-length ODN was incorporated into a polymer through radical acrylamide polymerization whereas failure sequences and other impurities were removed by washing. Pure full-length ODN was obtained by cleaving it from the polymer. In the second method, the failure sequences were capped by a methacrylated phosphoramidite in each synthetic cycle. During purification, the failure sequences were separated from the full-length ODN by radical acrylamide polymerization. The full-length ODN was obtained via water extraction. For both methods, excellent purification yields were achieved and the purity of ODNs was very 
satisfactory. Thus, this new technology is expected to be beneficial for large scale ODN purification. 


\section{Chapter 1}

\section{Introduction}

\subsection{General Introduction}

Oligodeoxynucleotide (ODN) is an essential molecule that has high anticipation for a wide range of applications including therapeutic development, ${ }^{1}$ ODN drug production, ${ }^{2}$ and biological studies. ${ }^{3}$ For decades, ODN has been successfully made by automated synthesis using phosphoramidite chemistry and solid phase synthesis. After the synthesis, crude ODN is cleaved from the solid support containing full-length ODN and impurities (small organic molecules and failure sequences). To obtain pure ODN, the crude product has to be purified before the applications.

The most widely used methods for ODN purification are gel electrophoresis and high performance liquid chromatography (HPLC). Although these methods work well in academic laboratory, they have disadvantages. They are suitable for small scale but limited for large scale ODN purification due to expensive instrument, large volume of harmful solvents, and intensive work.

From the problems described above, many researchers created alternative methods for ODN purification. Those are affinity purification, such as biotin-avidin extraction ${ }^{4}$ and fluorous chromatography. ${ }^{5}$ However, the methods still suffer from several drawbacks including high budget, labor, and timing. They are still not suitable for high-throughput purification. 


\subsection{The new ODN purification technology}

To overcome the problems, we have developed new ODN purification technology. The principle of the new technology utilizes polymerization reaction to separate full-length ODN from impurities or remove impurities from full-length ODN. Therefore, it does not involve any types of chromatography. The purification is accomplished by simple operations, such as shaking, and filtration.

There are many polymerization reactions which can be used for ODN purification, such as atom transfer radical polymerization (ATRP), ${ }^{6}$ click polymerization, ${ }^{7}$ and DielsAlder polymerization. ${ }^{8}$ However, the polymerization reaction that we decided to use is a radical acrylamide polymerization. The reaction is exact same as preparation of polyacrylamide gel for electrophoresis. It is commonly used in biology and biochemistry research. In addition, the reaction involves low-cost materials. Therefore, this reaction is suitable for large scale ODN purification. We have designed two simple nonchromatographic methods which are presented as follows.

For the first method, the concept of ODN purification is illustrated in Scheme 1.1. During automated synthesis, the full-length ODN will be attached with the designed phosphoramidite containing a terminal deoxynucleotide, a polymerizable function, and a cleavable linker at 5'-end. However, the failure sequences will not. After the synthesis, purification is achieved by polymerization of full-length ODN. Only full-length ODN is incorporated into the polymer while the failure sequences are not. Then, the failure sequences and other impurities are removed by washing with water. Finally, the pure fulllength ODN will be released by cleaving from the gel. 

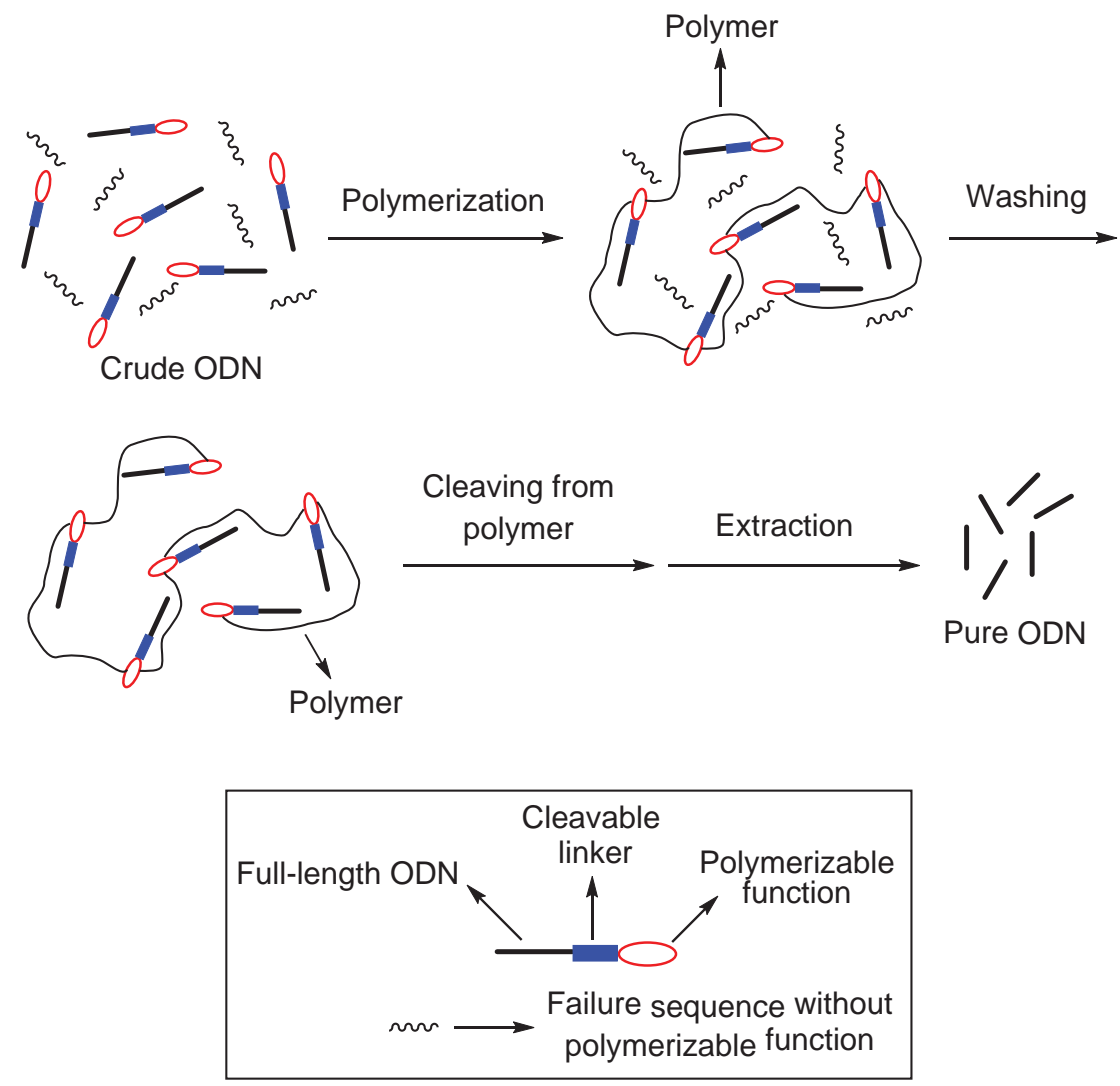

Scheme 1.1. General design of the first ODN purification method using catching fulllength ODN by polymerization 
The principle of the second method is to catch the failure sequences by polymerization. For each synthetic cycle, failure sequences are capped with the phosphoramidite consisting of polymerizable group when the full-length ODN is not. Only failure sequences are incorporated into the gel. Then, the pure full-length ODN are recovered by water extraction.
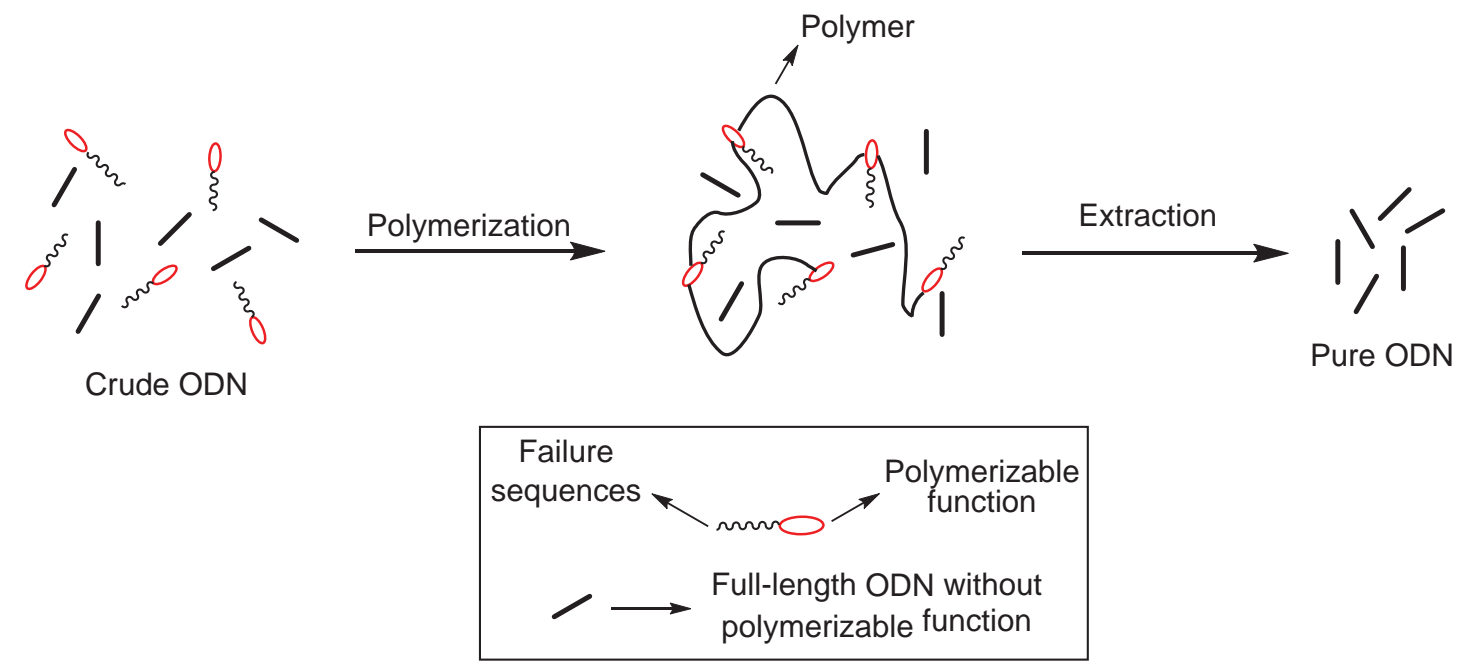

Scheme 1.2. General design of the second ODN purification method using catching failure sequences by polymerization

Two methods are complimentary to each other. For catching full-length ODN, it is suitable to purify long sequence ODN because it only requires the designed phosphoramidite for coupling at the last synthetic cycle. On the other hand, catching failure sequences is appropriate for purification of short sequence ODN since it is necessary to cap failure sequences with polymerizable group after each monomer addition. So, cleaving step is not required for the second method. 
According to both methods, we designed three methacrylated phosphoramidites for ODN synthesis. These compounds are vital molecules for ODN purification. They are stable under ODN synthesis conditions. The compound $\mathbf{1 . 1}$ is used for ODN purification by catching full-length ODN (The first method). The structure consists of the methacylamide group (polymerizable group) for polymerization, the diisopropyl silyl acetal group (cleavable linker), and thymidine as a terminal deoxynucleotide (Figure 1.1).

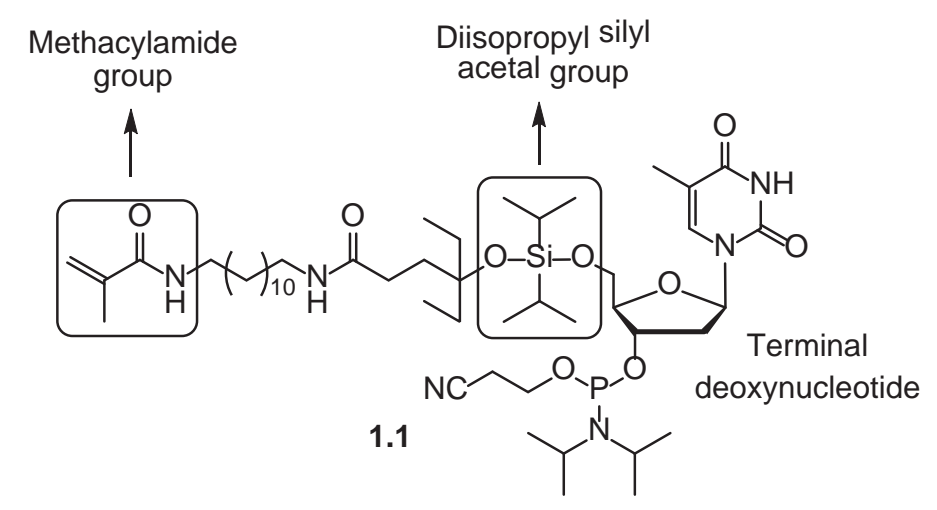

Figure 1.1. Phosphoramidite 1.1

The ODN synthesis using phosphoramidite $\mathbf{1 . 1}$ is shown in Scheme 1.3, the compound 1.1 will couple at $5^{\prime}-\mathrm{OH}$ of (n-1)-mer to get n-mer (1.4). The compound $\mathbf{1 . 1}$ will not be able to couple the failure sequences because the $5^{\prime}-\mathrm{OH}$ groups are capped with capping agent. After cleavage and deprotection, the crude mixture will contain $\mathbf{1 . 5}$, failure sequences, and small organic molecules. 

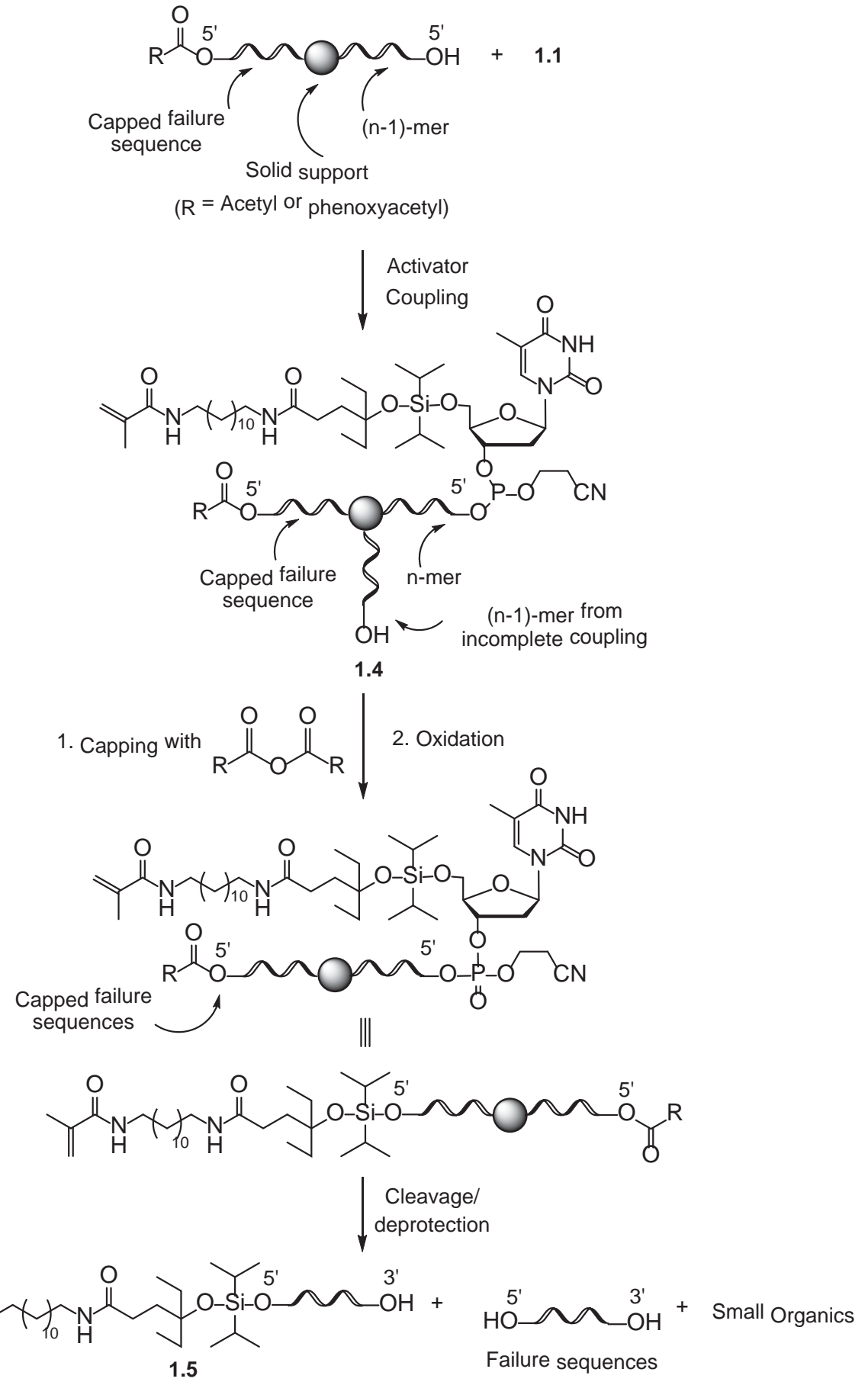

Scheme 1.3. ODN synthesis for catching full-length ODN 
As seen in Scheme 1.4, crude ODN will be polymerized by acrylamide radical polymerization. Only compound $\mathbf{1 . 5}$ which has methacrylamide group will be incorporated into the insoluble gel 1.6. Then, failure sequences and small organic molecules will be removed by washing with water. Cleaving the diisopropyl silyl acetal group of $\mathbf{1 . 6}$ will release pure full-length ODN 1.7.

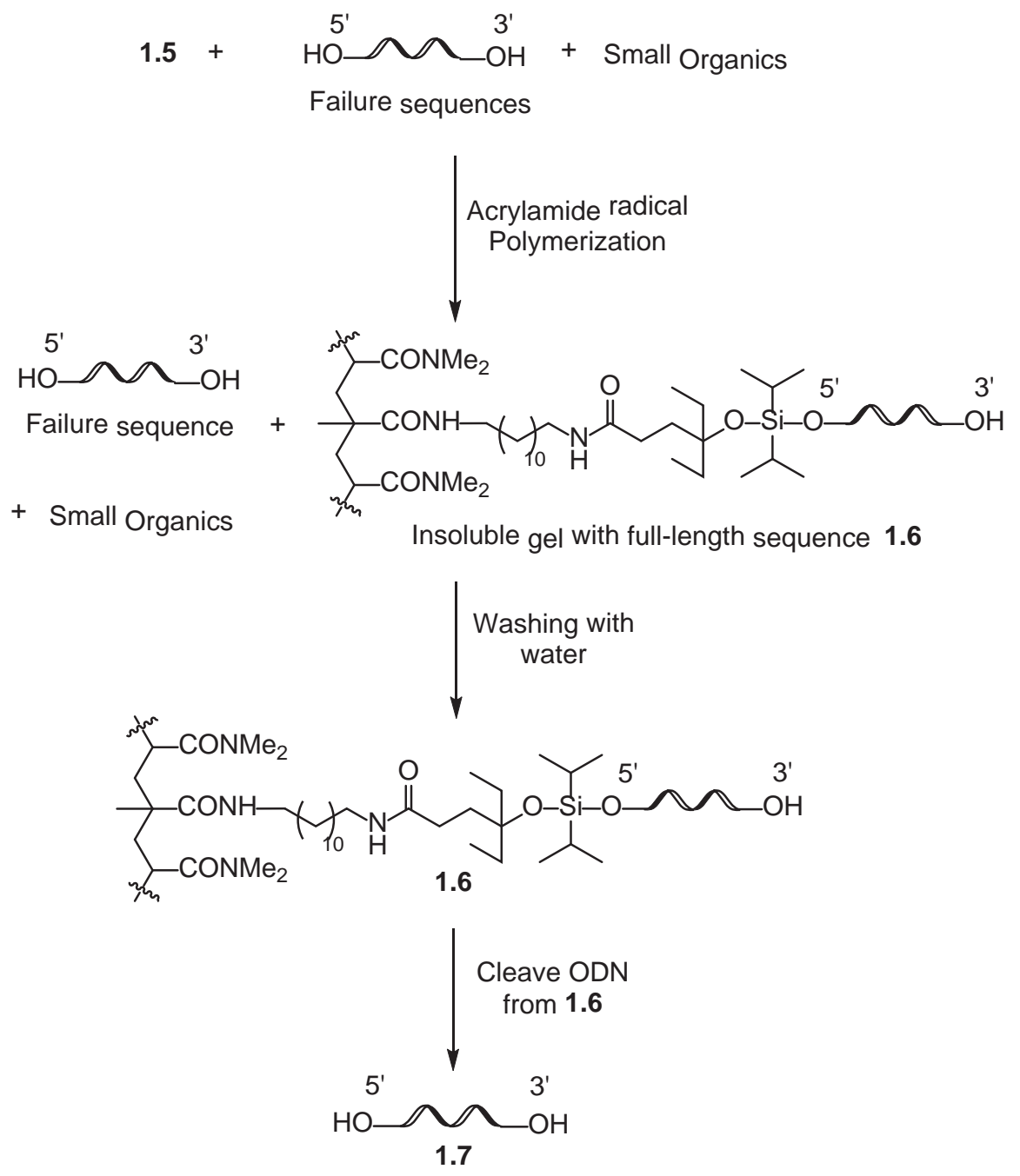

Scheme 1.4. Catching full-length ODN by polymerization 
The phosphoramidite $\mathbf{1 . 2}$ (Figure 1.2) containing only methacrylamide group is used as a capping agent to purify ODN by catching failure sequences (The second method). The method is illustrated in Scheme 1.5. Instead of normal capping agent, the failure sequences are capped with $\mathbf{1 . 2}$ to give 1.8. Since $5^{\prime}$-end of the full-length ODN is protected, it will not be capped by 1.2. After the synthesis, cleavage and deprotection will give crude mixture which comprises of failure sequences 1.9, full-length ODN 1.10, and small organic molecules.

During acrylamide radical polymerization, only $\mathbf{1 . 9}$ is polymerized into the gel to get insoluble gel (1.11) while the full-length ODN 1.10 is not. After that, water will be used to extract $\mathbf{1 . 1 0}$ from the gel. Then, $n-\mathrm{BuOH}$ precipitation will removes small organic molecules to give $\mathbf{1 . 1 0}$.

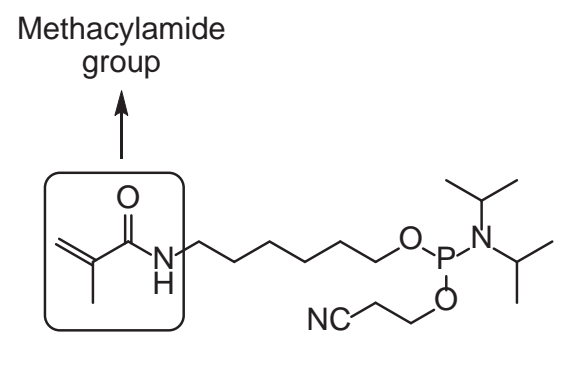

1.2

Figure 1.2. Phosphoramidite 1.2 

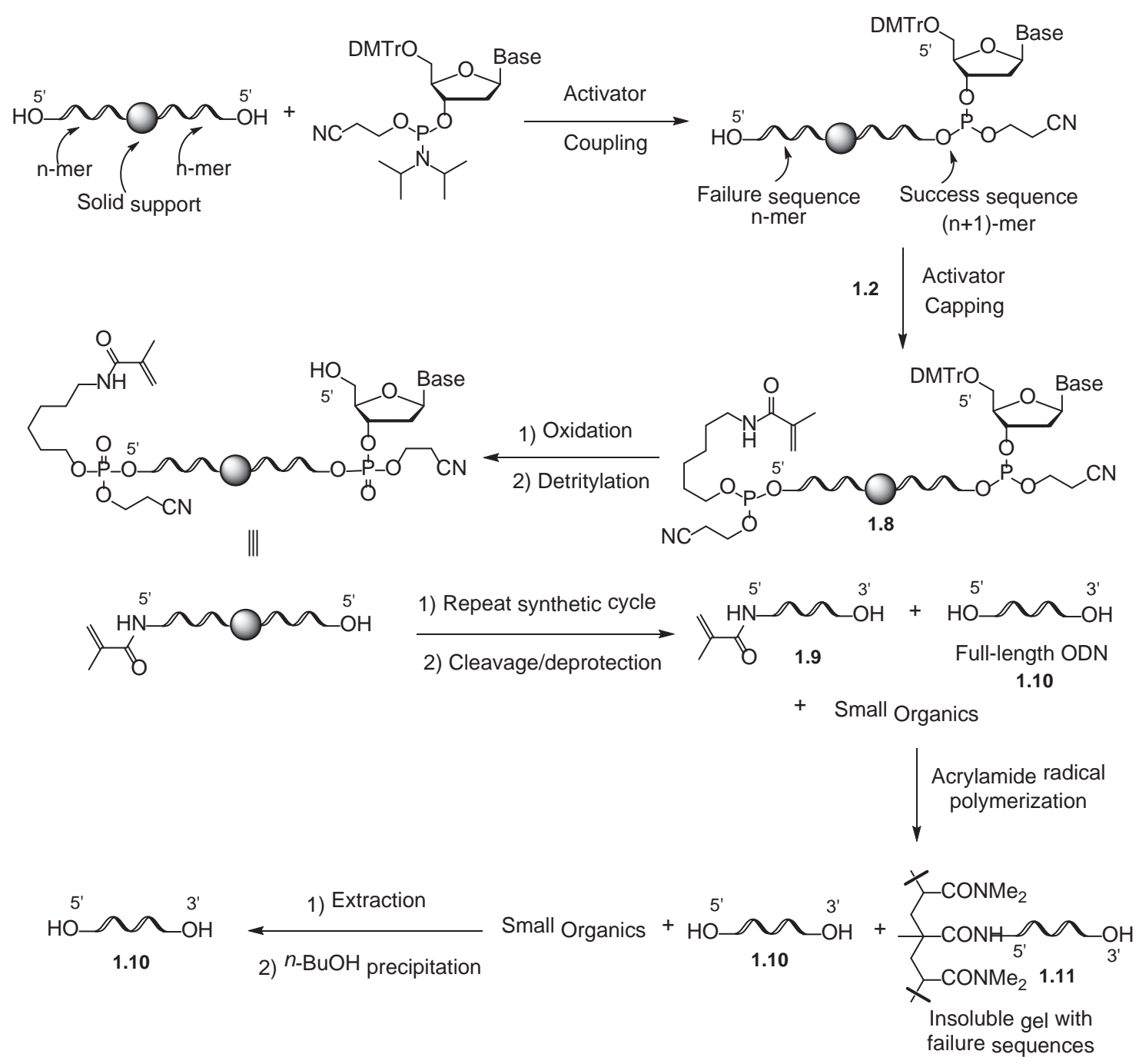

Scheme 1.5. Catching failure sequences by polymerization 
For the compound $\mathbf{1 . 3}$, the functional groups are similar to the compound $\mathbf{1 . 1}$ (The methacylamide group and the diisopropyl silyl acetal group). So, the method used for ODN purification is as same as the first ODN purification method. However, it has the bis(hydroxymethyl)malonate group which utilizes phosphate group to be linked at 5 '-end of ODN.

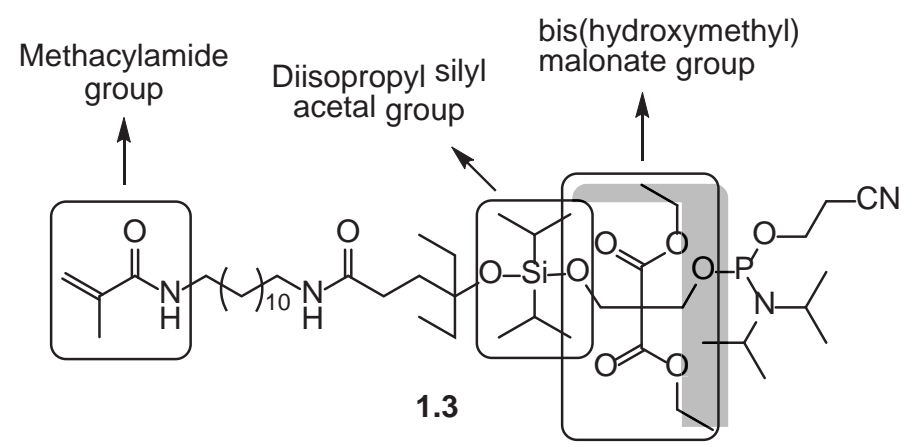

Figure 1.3. Phosphoramidite $\mathbf{1 . 3}$

The 5'-phosphorylated ODN synthesis using $\mathbf{1 . 3}$ is presented in Scheme 1.6. The compound 1.3 will couple with full-length ODN at $5^{\prime}$-end in the last synthetic cycle to get 1.12. On the other hand, the failure sequences will not be able to couple with $\mathbf{1 . 3}$ because the 5'-OH groups are already capped with capping agent. After cleavage and deprotection, the crude mixture will contain $\mathbf{1 . 1 3}$, failure sequences, and small organic molecules.

After the synthesis, crude ODN will be polymerized by polyacrylamide gel formation (Scheme 1.7). The ODN 1.13 will be polymerized into the insoluble gel 1.14. Then, water will be used to wash impurities. Next, cleaving the diisopropyl silyl acetal group will release ODN 1.15. Bis(hydroxymethyl)malonate on the phosphate group will be removed using ammonium hydroxide solution at $80{ }^{\circ} \mathrm{C}$. Lastly, $n$-BuOH precipitation will give pure $\mathbf{1 . 1 6}$ 

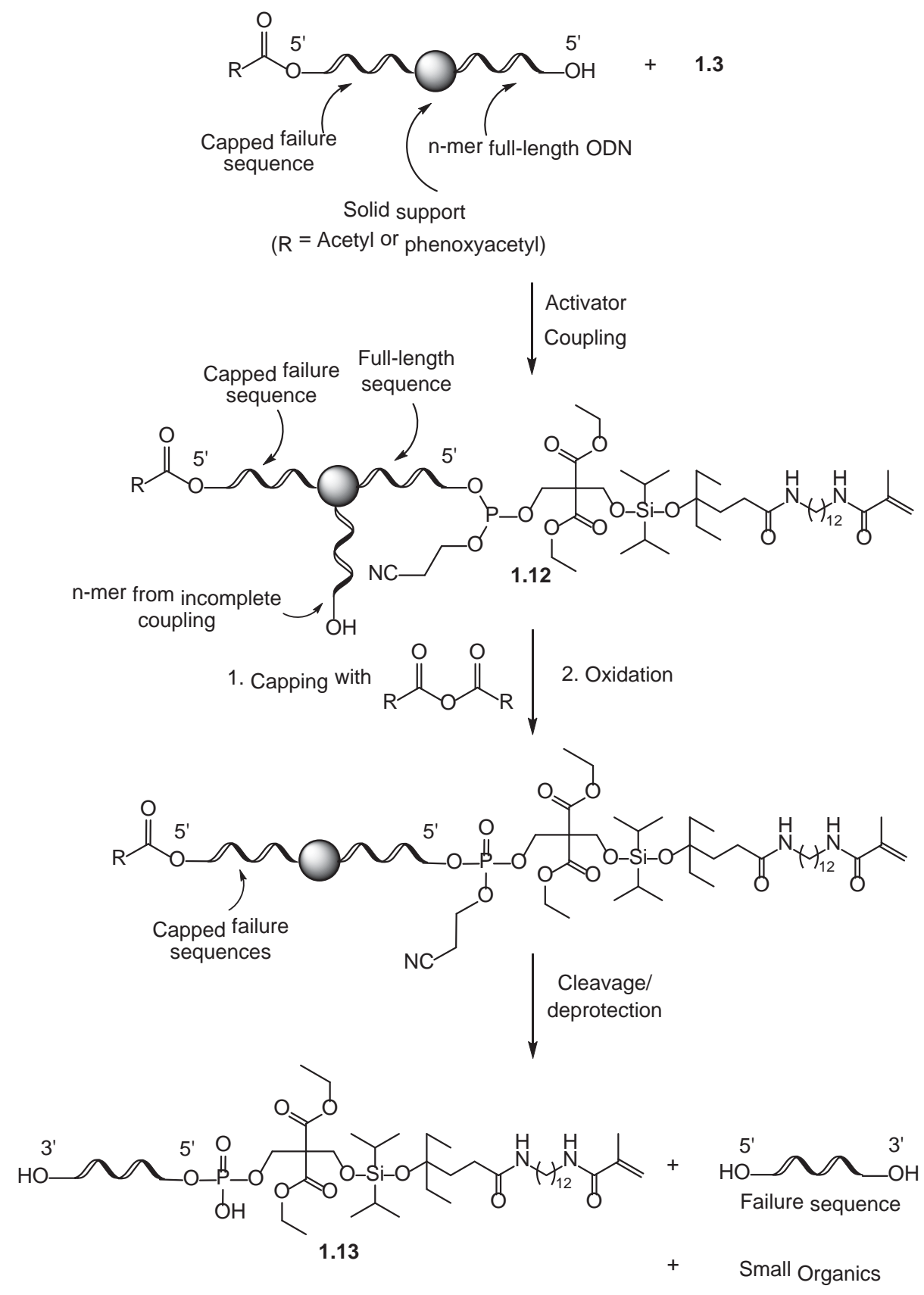

Scheme 1.6. 5'-Phosphorylated ODN synthesis for catching full-length ODN 


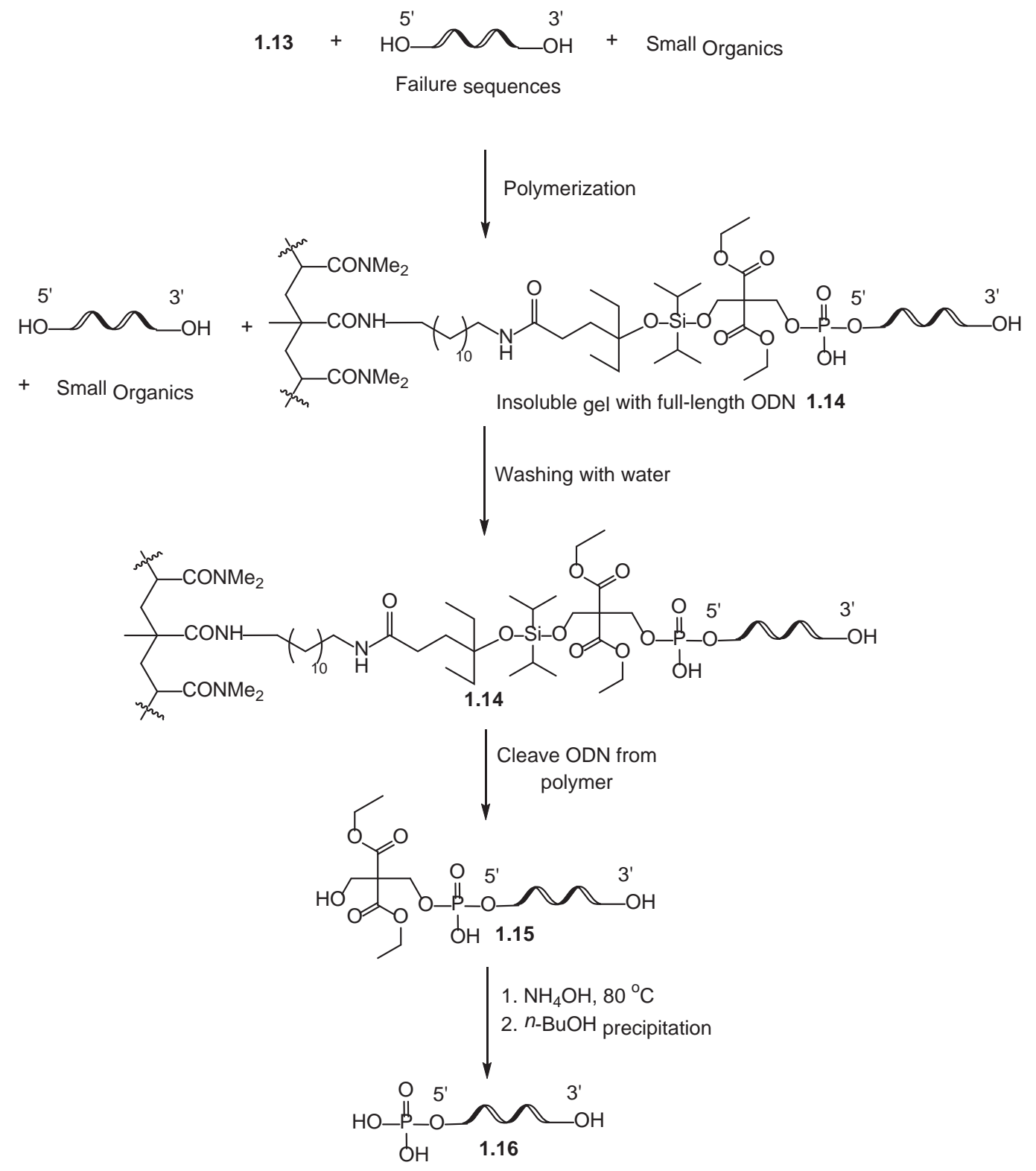

Scheme 1.7. Catching full-length 5 '-phosphorylated ODN by polymerization 
This dissertation focuses on our ODN purification technology. Background information about ODN including applications, synthesis, impurities during the synthesis, typical ODN purification methods, and ODN characterization is introduced in Chapter 2.

The specific task for the new ODN purification technology has been described in the subsequent chapters (Chapter 3-6). The synthesis of compound $\mathbf{1 . 1}$ and its purification method (Catching full-length ODN by polymerization) are reported in Chapter 3. The second purification method (Catching failure sequences by polymerization) using the capping agent 1.2 is explained in details in Chapter 4. Purification of 5'-phosphorylated ODN including the synthesis of phosphoramidite 1.3, long sequence ODN purification, and large-scale ODN purification using $\mathbf{1 . 3}$ is presented in Chapter 5. In addition, the future research plan with alternative acid-labile functional group for new phosphoramidite, further studies for catching failure sequences, and peptide purification is summarized in Chapter 6. 


\section{References}

1. Patil, S.; Rhodes, D.; Burgess, D., DNA-based therapeutics and DNA delivery systems: A comprehensive review. AAPS J 2005, 7 (1), E61-E77.

2. Thi Huyen, L.; Thi Thu Thuy, N.; Van Phuc, P.; Thi Minh Huyen, N.; Quang Huan, L., Using DNA nanotechnology to produce a drug delivery system. Advances in Natural Sciences: Nanoscience and Nanotechnology 2013, 4 (1), 015002.

3. (a) Wing, R.; Drew, H.; Takano, T.; Broka, C.; Tanaka, S.; Itakura, K.; Dickerson, R. E., Crystal structure analysis of a complete turn of B-DNA. Nature 1980, 287 (5784), 755-758; (b) Wang, A.; Quigley, G.; Kolpak, F.; van der Marel, G.; van Boom, J.; Rich, A., Left-handed double helical DNA: variations in the backbone conformation. Science 1981, 211 (4478), 171-176; (c) Shakked, Z.; Rabinovich, D.; Cruse, W. B. T.; Egert, E.; Kennard, O.; Sala, G.; Salisbury, S. A.; Viswamitra, M. A., Crystalline A-DNA: The XRay Analysis of the Fragment d(G-G-T-A-T-A-C-C). Proceedings of the Royal Society of London. Series B. Biological Sciences 1981, 213 (1193), 479-487.

4. Fang, S.; Bergstrom, D. E., Fluoride-cleavable biotinylation phosphoramidite for 5'-end-labeling and affinity purification of synthetic oligonucleotides. Nucleic Acids Research 2003, 31 (2), 708-715.

5. Pearson, W. H.; Berry, D. A.; Stoy, P.; Jung, K.-Y.; Sercel, A. D., Fluorous Affinity Purification of Oligonucleotides. The Journal of Organic Chemistry 2005, 70 (18), 71147122.

6. (a) Patten, T. E.; Matyjaszewski, K., Atom Transfer Radical Polymerization and the Synthesis of Polymeric Materials. Advanced Materials 1998, 10 (12), 901-915; (b) 
Matyjaszewski, K.; Xia, J., Atom Transfer Radical Polymerization. Chemical Reviews 2001, 101 (9), 2921-2990.

7. Qin, A.; Lam, J. W. Y.; Tang, B. Z., Click polymerization. Chemical Society Reviews 2010, 39 (7), 2522-2544.

8. (a) Stille, J. K.; Anyos, T., A novel Diels-Alder polymerization. Journal of Polymer Science Part A: General Papers 1964, 2 (3), 1487-1491; (b) Kumar, U.; Neenan, T. X., Diels-Alder Polymerization between Bis(cyclopentadienones) and Acetylenes. A Versatile Route to New Highly Aromatic Polymers. Macromolecules 1995, 28 (1), 124-130. 


\section{Chapter 2}

\section{History and Background}

\subsection{Deoxyribonucleic acid}

Deoxyribonucleic acid (DNA) is the molecule that contains genetic information for development and growth of living cells. DNA was discovered in 1869 by Friedrich Miescher. ${ }^{1}$ He reported that there was an unknown material isolated from cell's nuclei. The properties of the substance were not as same as proteins or any recognized molecules. For example, protease pepsin could not digest this compound. Elemental analysis showed the material contains carbon, nitrogen, oxygen, and hydrogen. However, it did not contain sulfur but significant amount of phosphorus. In addition, the compound was dissolved in basic solution and was precipitated in acidic or alkaline solutions. According to the isolation the material from the cell nuclei, he named DNA "nuclein". He and his coworkers, Felix Hoppe-Seyler and P. Plosz, published the manuscript in 1871. After the discovery, Miescher continued analyzing DNA from animal's cells and he found very interesting information. For instance, he was able to confirm that nuclein is in the form of phosphoric acid. Furthermore, he could estimate the atomic formula of nuclein in salmon sperm. Despite his success to obtain the analyses, Miescher never returned to find the conclusion of nuclein.

Since Miescher's discovery, some scientists were interested to investigate more information about DNA. In 1890s, Albrecht Kossel isolated four nucleobases: adenine, cytosine, guanine, and thymine which are DNA's key components. Later, Phoebus Levene was able to identify that each unit of DNA contains nucleobase and 2'-deoxyribose. In 
1953, James Watson and Francis Crick proposed three dimensional structure of DNA based on the X-ray diffraction image (Figure 2.1). ${ }^{2}$ The picture was taken by Rosalind Franklin and Maurice Wilkins. They found that there are two strands of DNA existing in the form of double helix (Figure 2.2).

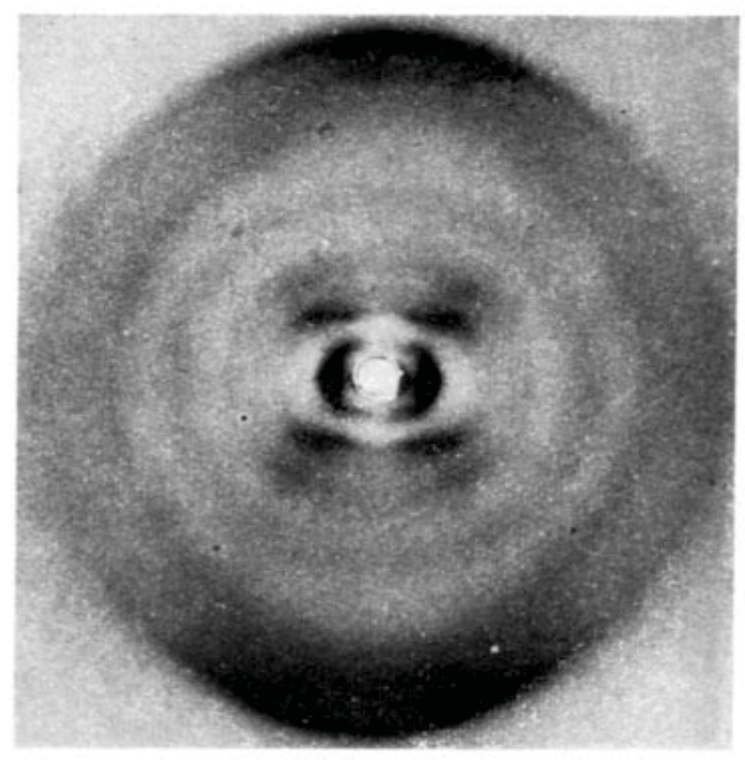

Figure 2.1. X-ray diffraction image of DNA [Reprinted by permission from Macmillan Publishers Ltd: Nature 1953, 171 (4356), 737-738, copyright 1953. See Appendix D. Permission from publishers.]

Double-stranded DNA consists of two deoxyribonucleic acid strands. Each strand has the backbone of deoxynucleotide. Each deoxynucleotide contains deoxynucleoside and phosphate group. The nucleoside comprises of 2'-deoxyribose (pentose sugar) and heterocyclic nucleobase (Figure 2.3). ${ }^{3}$ The nucleobase and deoxyribose are connected together with $\beta$-glycosidic bond. There are two types of nucleobases in DNA: purines and 
pyrimidines (Table 2.1). Purines are the heterocyclic compounds containing both being fused fived- and sixed-membered rings. They are adenine (A) and guanine (G).

Pyrimidines are sixed-membered ring heterocyclic compounds. They are cytosine (C) and thymine (T). Each deoxynucleotide has bonded with phosphate diester at 5' hydroxyl group to attach one unit and 3' hydroxyl group to attach the next unit. Both DNA strands have the specific sequences which are complementary to each other. They are held together by two weak forces: hydrogen bonding and nucleobase stacking interaction. Each nucleobase has the specific base pairing and number of hydrogen bonds. For example, adenine (A) and thymine (T) are formed with two hydrogen bonds, whereas cytosine (C) and guanine (G) are formed with three hydrogen bonds (Figure 2.4). ${ }^{4}$

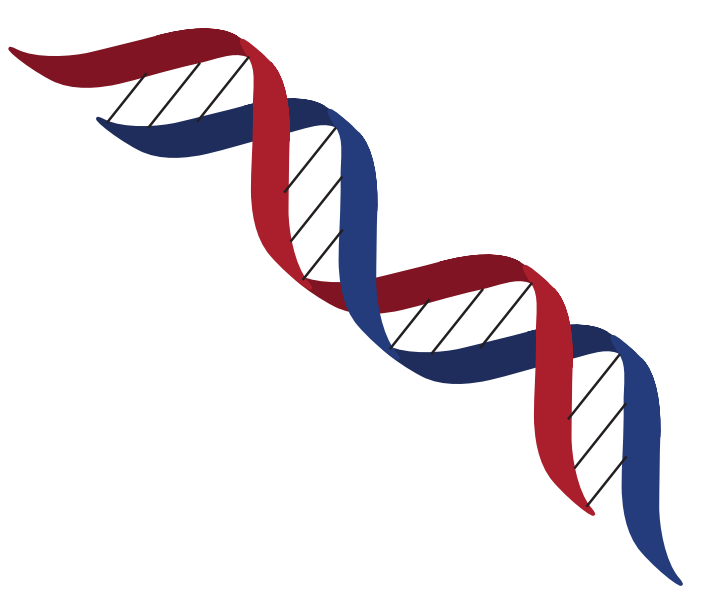

Figure 2.2. Double helical structure of DNA 


\section{Nucleosides}<smiles>Nc1ncnc2c1ncn2C1COCC(O)C1</smiles>

Adenosine<smiles>Nc1ccn(C2COC(CO)C(O)C2)c(=O)n1</smiles>

Cytosine<smiles>Nc1nc2c(ncn2C2COC(CO)C(O)C2)c(=O)[nH]1</smiles>

Guanosine<smiles>Cc1cn(C2COC(CO)C(O)C2)c(=O)[nH]c1=O</smiles>

Thymidine
Nucleotides

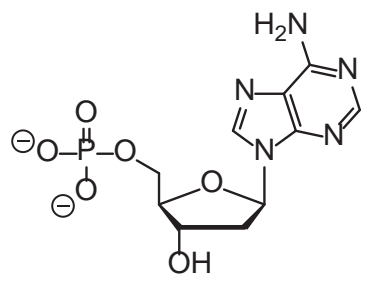

Adenosine-5'-monophoshate<smiles>Nc1nc2c(ncn2C2CC(O)C(COP(=O)([O-])[O-])O2)c(=O)[nH]1</smiles>

Cytosine-5'-monophoshate<smiles>Nc1ccn(C2CC(O)C(COP(=O)([O-])[O-])O2)c(=O)n1</smiles>

Guanosine-5'-monophoshate

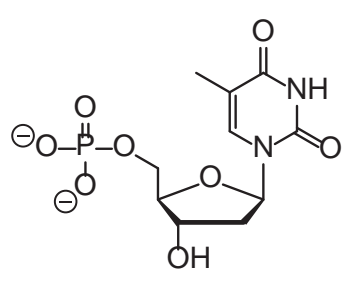

Thymidine-5'-monophoshate

Figure 2.3. Nucleosides and Nucleotides in DNA 


\begin{tabular}{|l|l|}
\hline Purine & Pyrimidine \\
\hline
\end{tabular}

Table 2.1. Purine and pyrimidine bases in DNA
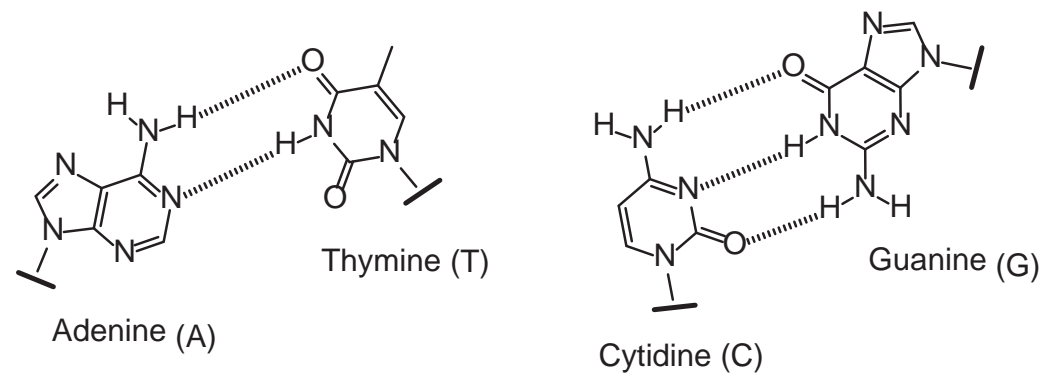

Figure 2.4. Hydrogen bonding for each base pair in double-stranded DNA 


\subsection{Oligodeoxynucleotide and applications}

Oligonudeoxycleotide (ODN) is short single-stranded DNA. It has been widely used in medical and scientific research. Examples include antisense ODN drug, DNA sequencing, Polymerase Chain Reaction (PCR), and synthetic biology for total gene synthesis.

\subsubsection{Antisense ODN}

The concept of antisense ODN is based on DNA-DNA or DNA-mRNA hybridization. The antisense sequence is designed and synthesized to selectively hybridize to the complementary strand of DNA or mRNA (It is also called sense sequence). ${ }^{5}$ After binding the target sequence, transcription or translation process will be terminated. This can suppress abnormal gene expression that can cause diseases. There are two ODN drugs on the market. Fomivirsen (Commercial name is Vitravene) is the first one that was approved by United States Food and Drug Administration (FDA) in 1998. Fomivirsen contains 21-mer oligodeoxynucleotides (Figure 2.5.1) that can be used to cure Cytomegalovirus retinitis (Inflammatory retina). ${ }^{6}$ The structure contains phosphothiorate diester bond instead of phosphate diester bond. So, the compound is resistant to degradation by nucleases. In addition, Mipomersen (Commercial name is Kynamro) is the other antisense ODN drug that FDA has been approved in 2013. This drug has a specific sequence to hybridize to messenger RNA of Apolipoprotein B. ${ }^{7}$ Therefore, it can reduce Familial hypercholesterolemia (Cholesterol in blood stream). 
The structure of Mipomersen contains 2'-deoxyribosenucleotides and 2'-Omethoxyethyl-modified ribonucleotides linked with phosphothiorate bonds (Figure 2.5.2). It's commercially available in a form of sodium phosphothiorate.

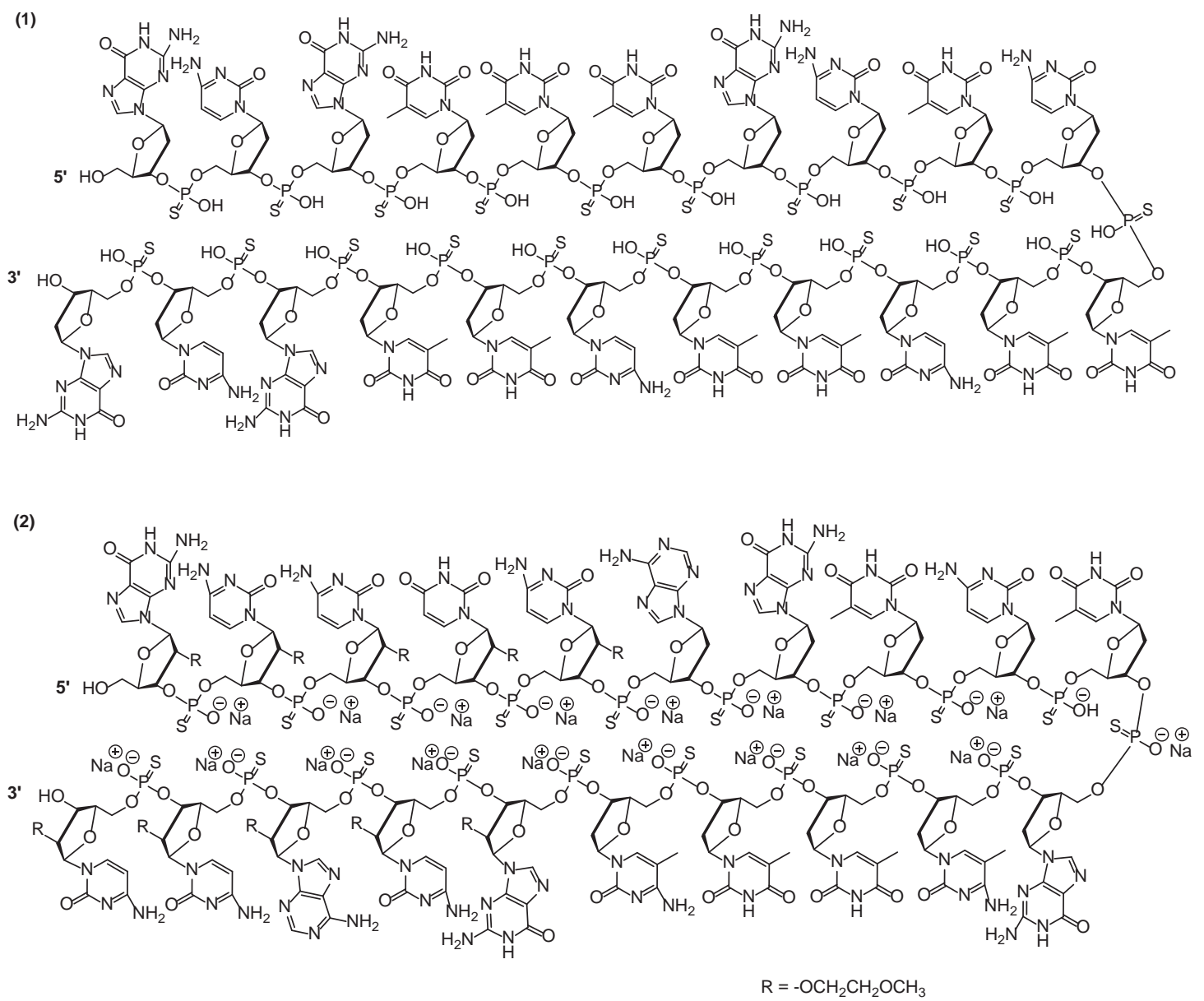

Figure 2.5.(1) structure of Fomivirsen (5'-GCG TTT GCT CTT CTTCTT GCG-3'), (2) structure of Mipomersen (5'-G*-C*-C*-U*-C*-AGTCTG-d(5-methyl-C)-TT-d(5methyl-C)-G*-C*-A*-C*-C*-3')[* = 2'-O-(2-methoxyethyl)] 


\subsubsection{DNA sequencing}

Another important application of ODN is DNA sequencing. DNA sequencing is to determine the order of deoxynucleotides in DNA sequence. One of the DNA sequencing methods that requires ODN is the Sanger method. ${ }^{8}$ It was created by Frederick Sanger and co-workers in 1977. The DNA sample is divided into four portions. Each portion contains ODN primers, DNA polymerase, and four standard deoxynucleosidetriphosphates (dNTPs) (dNTPs are dATP, dGTP, dCTP, and dTTP). However, only one of the modified dideoxynucleosidetriphosphates (ddNTP) (ddNTP is ddATP, ddGTP, ddCTP, or ddTTP) is added to each portion. These ddNTP are radioactively labeled or fluorescently labeled. The ODN primers hybridize to the target sequence. Then, DNA is elongated by coupling to dNTPs with polymerase catalysis. During elongation, ddNTP, which does not have 3'$\mathrm{OH}$ group, is also incorperated into DNA, so it ceases the elongation. Heat-initiated denaturation releases DNA fragments from target strand. Those fragments are separated by gel electrophoresis. With the gel image of the different fragments from each portion, the target sequence of DNA is determined.

\subsubsection{Polymerase chain reaction $(\mathrm{PCR})$}

PCR also has to use ODN as a primer. It is a commonly used technique that can amplify DNA by producing numerous copies of a specific DNA sequence. This technology had been established in 1983 by Kary Mullis. ${ }^{9}$ The concept of PCR is to synthesize a complementary DNA strand according to the template strand. First of all, double-stranded DNA is heated to separate the two template strands. After that, primers (short ODN sequences that are complementary to the $3^{\prime}$ segment of the template sequences) bind to each template sequence at $3^{\prime}$-end. The next process is called elongation. DNA polymerase 
synthesizes complementary DNA on the template sequence. Each new deoxynucleotide is added from $3^{\prime}$-end to $5^{\prime}$-end. Then, the process is terminated when the synthesis has reached to 5 -end. The process is usually repeated for multiple times. After PCR, a specific DNA sequence will be gathered in million to billion copies.

\subsubsection{Total gene synthesis}

Total gene synthesis requires thousands of ODNs. Gene synthesis is an important application for many areas of DNA technology, such as mutagenesis studies, gene expression, and gene engineering, etc. It is the method to make artificial genes using a combination of chemical reactions and biological technique. Since genes consists of very long double-stranded DNA, the synthesis needs to be partial ODN synthesis before assembling together. Firstly, sets of uniquely designed ODN segments are synthesized using solid phase synthesis with phosphoramidite chemistry. After ODNs are purified, each ODN segment is connected to each other using polymerase and precise annealing to get a

complete genes. ${ }^{10}$ Currently, some researchers use PCR to improve the method which lower the cost and errors during the synthesis. ${ }^{11}$

Furthermore, many ODN drug development projects are still underway. Large quantities of ODN have been highly demanded. As a result, ODN synthesis is essential for this research area as well. 


\subsection{ODN Synthesis}

Due to the high demand of ODN, synthesis of ODN has become important. In the history of ODN synthesis, many scientists were interested to develop ODN synthesis methods using chemical reactions. Some methods have been successful and people are still using them. However, some of them have drawbacks and people are no longer using those methods. The history of ODN synthesis is briefly summarized below.

\subsubsection{First ODN Synthesis Method}

Todd and Michelson published the first ODN synthesis method in $1955 .^{12}$ They reported the synthesis of dithymidyldeoxynucleotide using phosphorylchloridate method (Scheme 2.1). In the reaction, a phosphate linkage between two thymidines using 5'benzoyl-3'-phosphonyl thymidine (2.4) and 3'-benzoyl thymidine (2.5) in the presence of 2,6-lutidine was formed. The mechanism of the synthesis is illustrated in Scheme 2.2. First, 3'-hydroxyl group of $\mathbf{2 . 1}$ was phosphonated with benzyl phosphonic diphenyl phosphoric anhydride to get $H$-phosphonate 2.2. The compound 2.2 can be tautomerized to get hydroxyphosphite $\mathbf{2 . 3}$ and was then converted to $\mathbf{2 . 4}$ using $N$-chlorosuccinimide. Next, 5'hydroxyl group of $\mathbf{2 . 5}$ was coupled with the compound $\mathbf{2 . 4}$ to obtain $\mathbf{2 . 6}$ as a product. The synthesis appeared to be interesting. Nevertheless, there were some flaws in this method. In their report, the reaction between $\mathbf{2 . 4}$ and 2.5 was indeed slow. In addition, the phosphoryl chloride $\mathbf{2 . 4}$ was not stable since it could be hydrolyzed in the presence of moisture to get phosphoric acid. Therefore, 2.4 had to be generated in situ prior to coupling. 

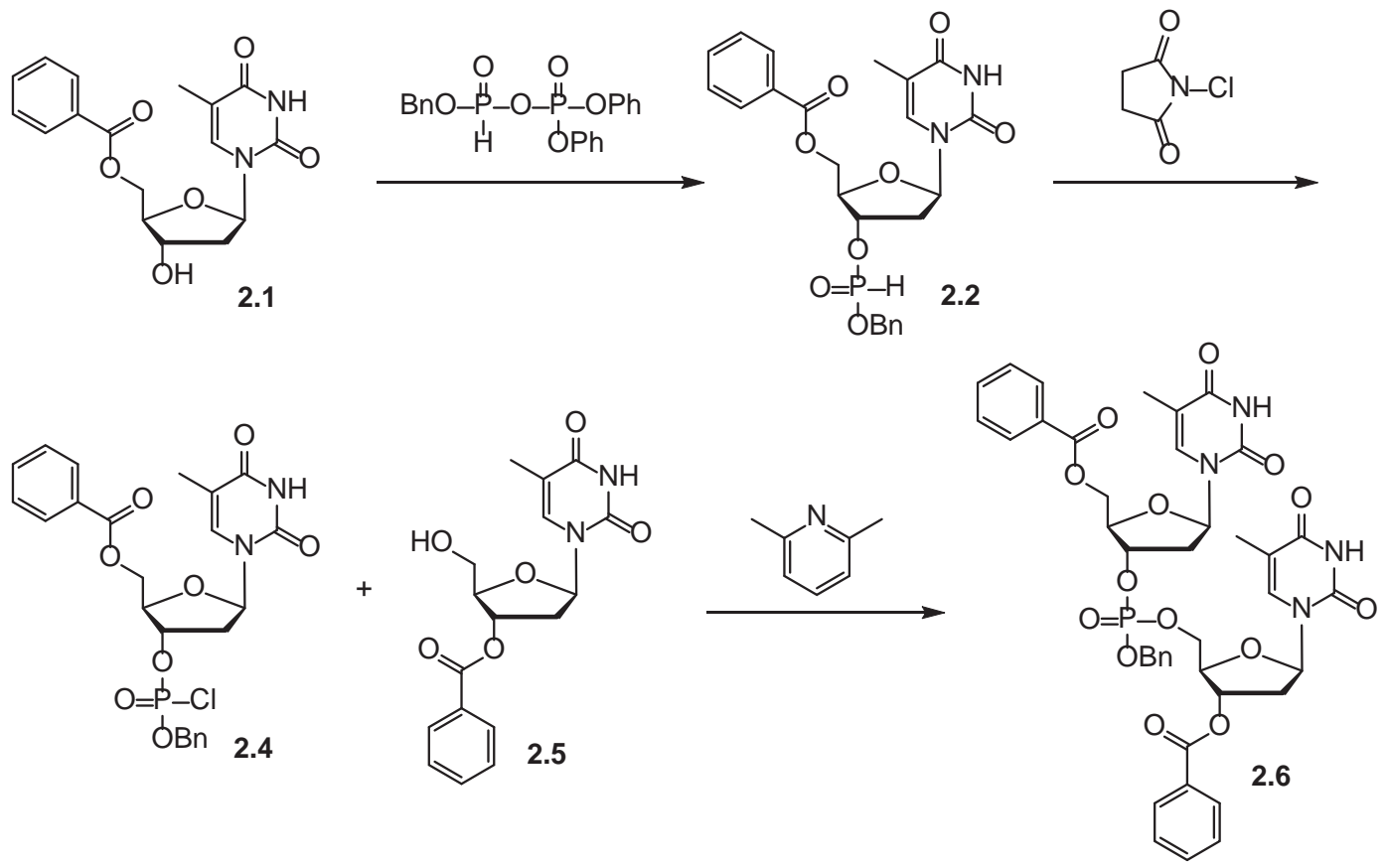

Scheme 2.1. First ODN Synthesis 

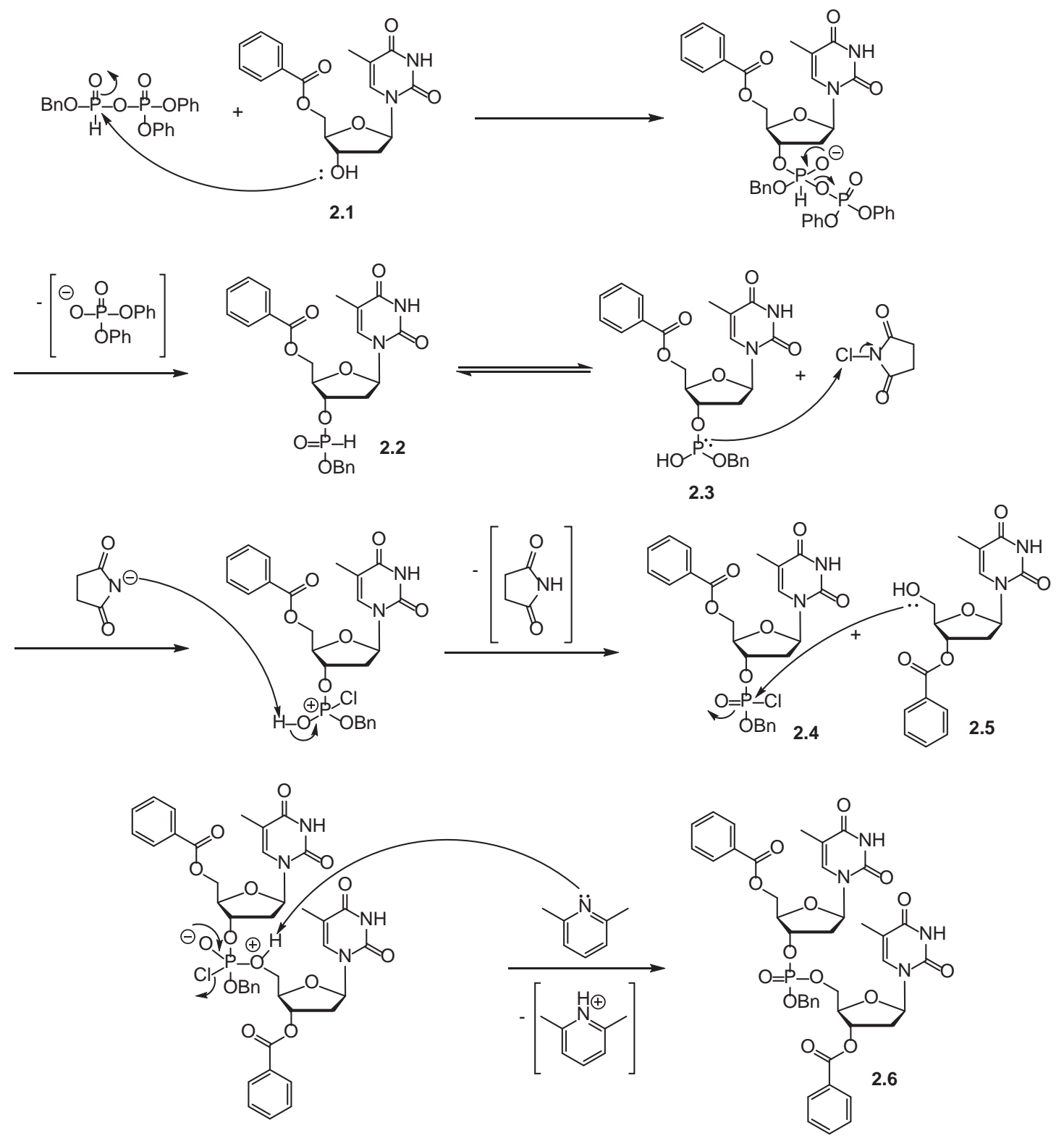

Scheme 2.2. Mechanism of the first ODN Synthesis 


\subsubsection{Phosphodiester Method}

In 1958, Khorana and his colleagues had developed the ODN synthesis after Todd and Michelson's work. They introduced the phosphodiester method to synthesize ODN instead of preparing the hydrolysable phosphochloridates. ${ }^{13}$ Later, they published the synthesis of 72-mer transfer ribonucleic acid (tRNA) using this method in 1970. The concept of the reaction is similar to a peptide coupling reaction. It is a condensation between reactants such as $5^{\prime}-O$-DMTr deoxynucleoside-3'-O-phosphate 2.7 and deoxynucleoside 2.9 using dicyclohexylcarbodiimide (DCC) as a coupling agent (Scheme 2.3).

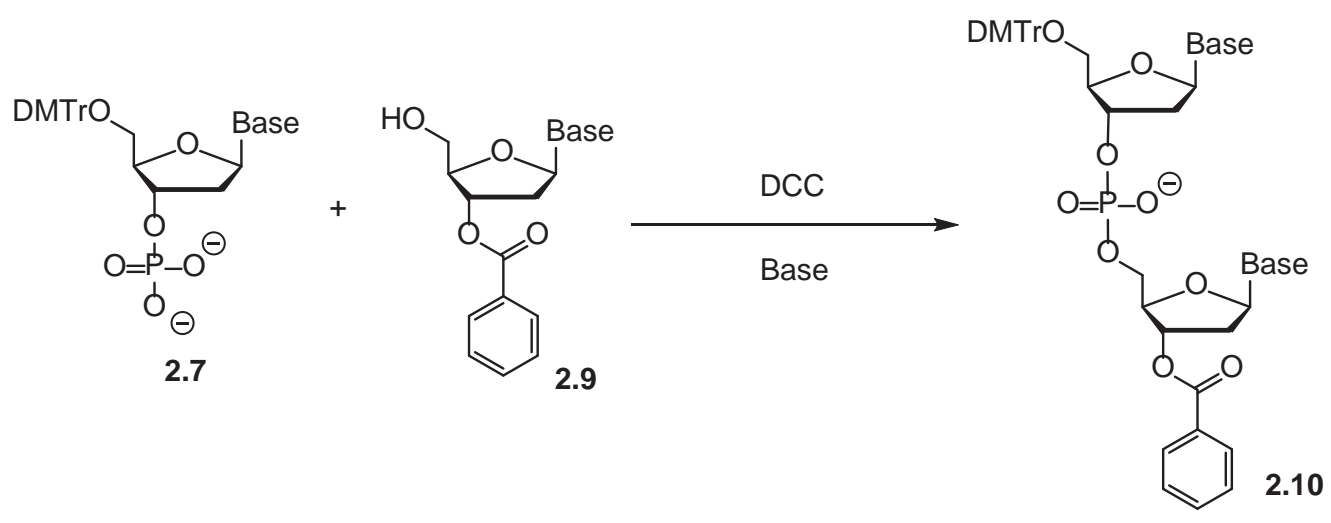

Scheme 2.3. ODN synthesis using phosphodiester method

The mechanism is shown in Scheme 2.4. The phosphate group of $\mathbf{2 . 7}$ is activated with DCC to get the intermediate 2.8. Then, the free 5'-hydroxyl group of $\mathbf{2 . 9}$ is coupled with $\mathbf{2 . 8}$ to give dideoxynucleoside phosphate $\mathbf{2 . 1 0}$ and 1,3-dicyclohexylurea (2.11) as a side product. 

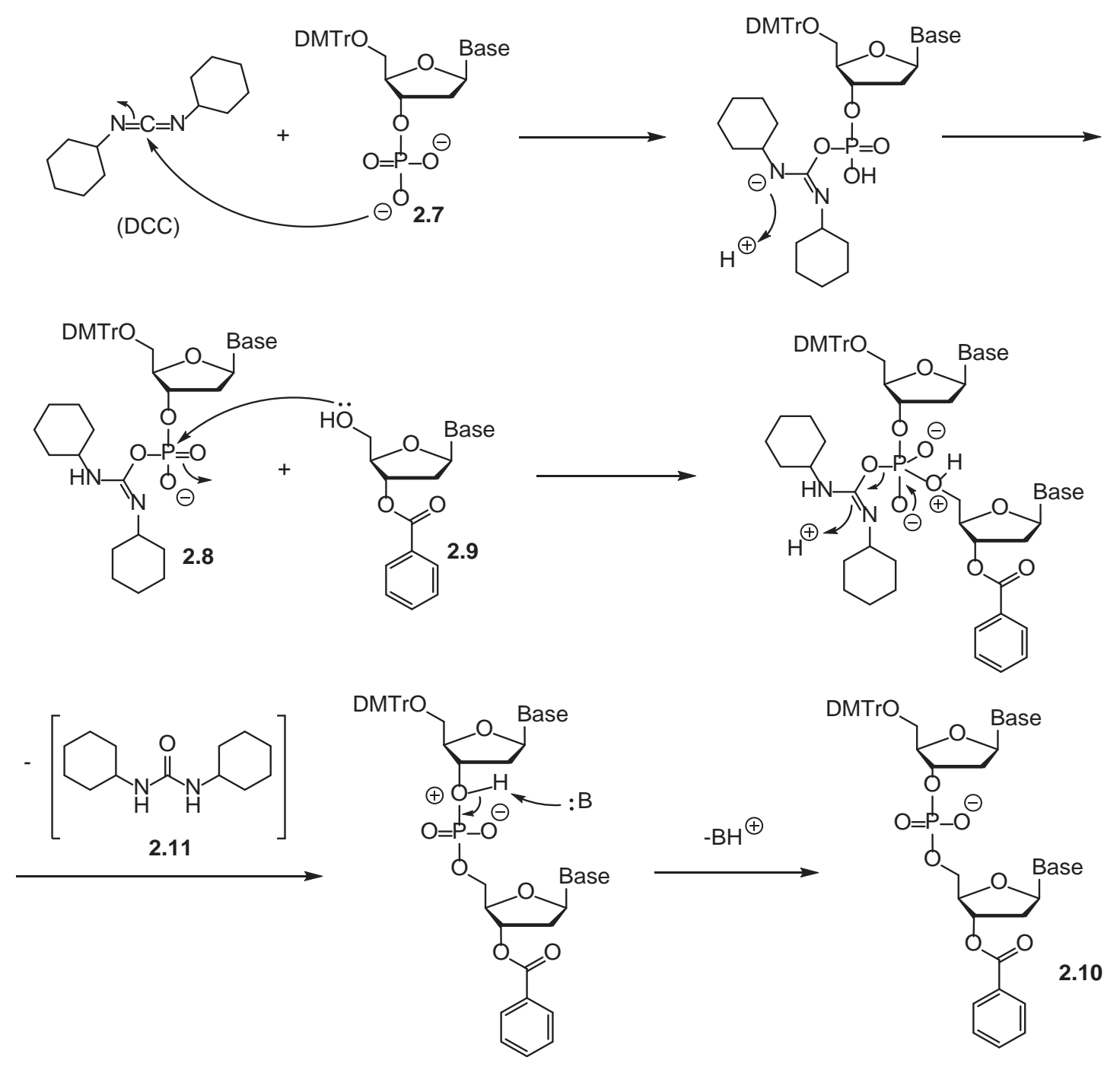

Scheme 2.4. Mechanism of ODN synthesis using phosphodiester method

Despite their success, Khorona and co-workers had found shortcomings. For instance, branched ODN was a major side-product during the synthesis. It was generated from the coupling between interdeoxynucleotide phosphate linkages and intermediate $\mathbf{2 . 8}$ (Scheme 2.5). Consequently, a laborious multi-step purification process was followed to remove branched contaminants after coupling. The other drawback was the long coupling 
time. They reported that coupling reaction between 2.8 and 2.9 took up to two days to get a good yield. That would be a serious problem when this method was used to synthesize long strand ODN.

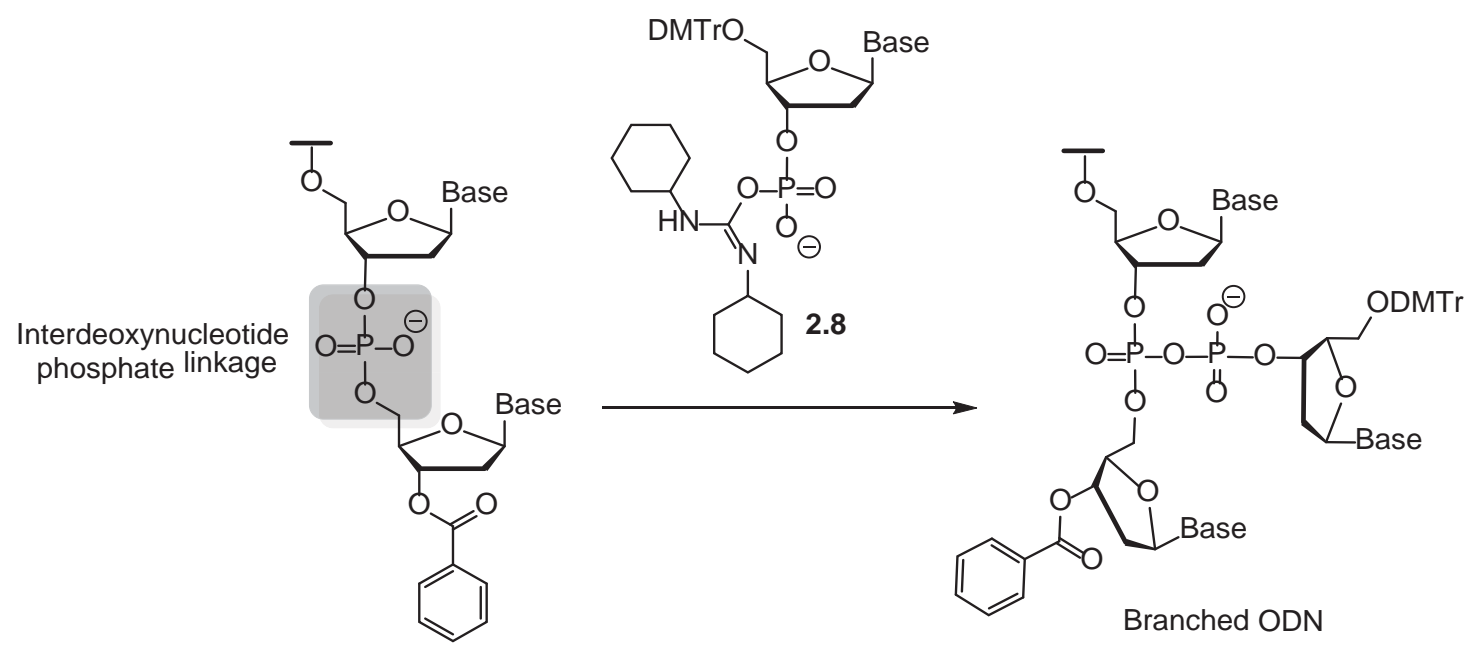

Scheme 2.5. Branched ODN from phosphodiester synthesis 


\subsubsection{Introduction of Solid Support for ODN Synthesis}

After successful peptide synthesis, solid-phase chemistry had been applied to the ODN synthesis. Robert Letsinger firstly introduced dimer and trimer ODN synthesis using solid support in 1963. In his paper, styrene-divinyl benzene polymer was used as a solid support to attach deoxycytidine (Scheme 2.6 ).${ }^{14}$ Since 3 '-hydroxyl group and $5^{\prime}$-hydroxyl group of $\mathbf{2 . 1 2}$ were protected with benzoyl group and DMTr group respectively, the amino group was coupled with $\mathbf{2 . 1 3}$ to give 2.14. ODN could be synthesized on this support by removal of DMTr group. Then, $5^{\prime}$-hydroxyl group would be ready for the next coupling with the other deoxynucleotide. Letsinger used the phosphotriester method for the synthesis. After the synthesis is complete, ODN was cleaved from the support with a strong base.

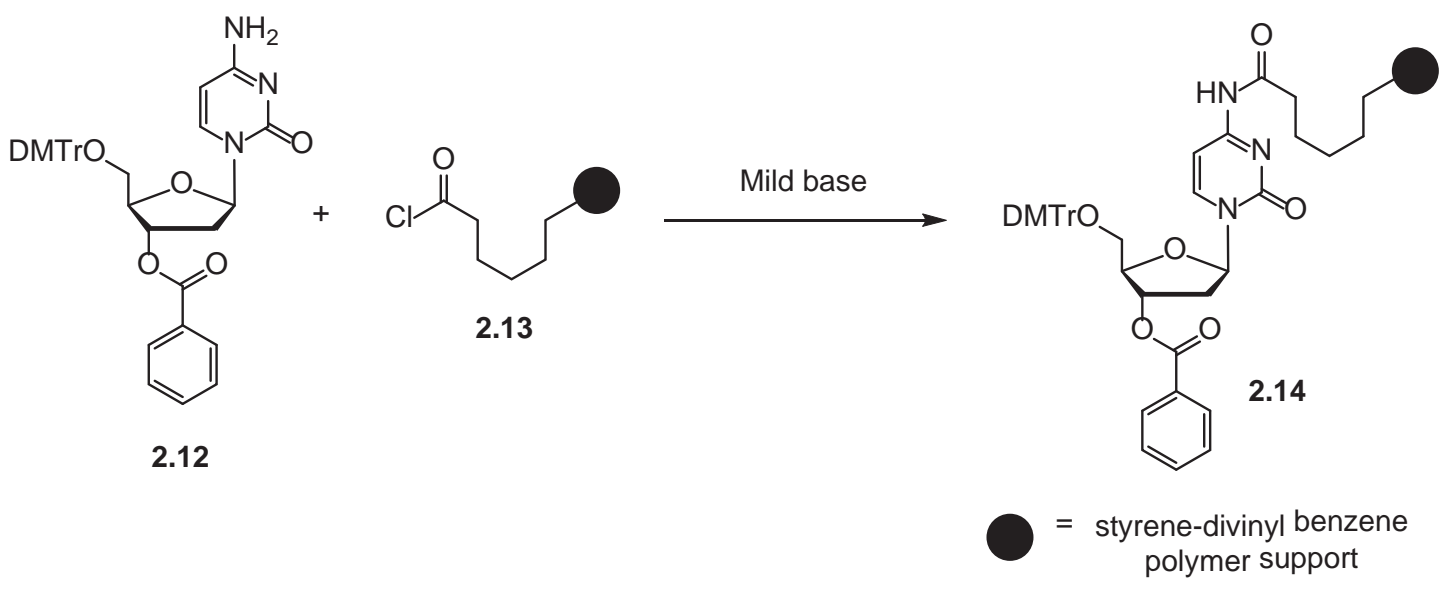

Scheme 2.6. Preparation of solid support using 5'-DMTr-protected dC. 
Later, Letsinger found the best way to attach the initial nucleoside to the solid support. Instead of attaching to the amino group of nucleobase, the solid support was linked to the 3 '-hydroxyl group of the nucleoside via an ester bond (Figure 2.6). Since the ester bond is more easily cleaved than the amide bond of the previous solid support (see 2.14), the new approach has been commonly used until nowadays. However, support swelling in organic solvent was still a main issue of the support.

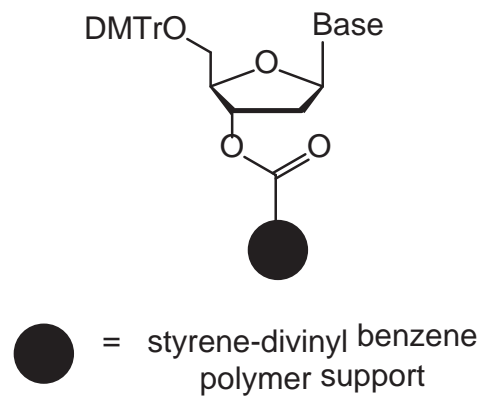

Figure 2.6. The new version of solid support attaching to 3 '-end of the initial nucleoside 


\subsubsection{Phosphotriester Method}

Phosphotriester method was published in 1969 by Letsinger and Ogilvie. ${ }^{15}$ The reaction is a coupling between reactants such as $5^{\prime}-O$-DMTr-deoxynucleoside $\mathbf{2 . 1 5}$ and deoxynucleoside-linked solid support $\mathbf{2 . 1 8}$ (Scheme 2.7). Mesitylsulfonyl nitrotriazole 2.16 (MSNT) was used as the coupling agent. The synthesis is similar to phosphodiester method, but the advantage is that the phosphate group $\mathbf{2 . 1 6}$ was protected by a 2 -cyanoethyl group. This avoided branched ODN which was generated in the previous version of phosphodiester method. In addition, 2-cyanoethyl group was simply removed with ammonium hydroxide $\left(\mathrm{NH}_{4} \mathrm{OH}\right)$.

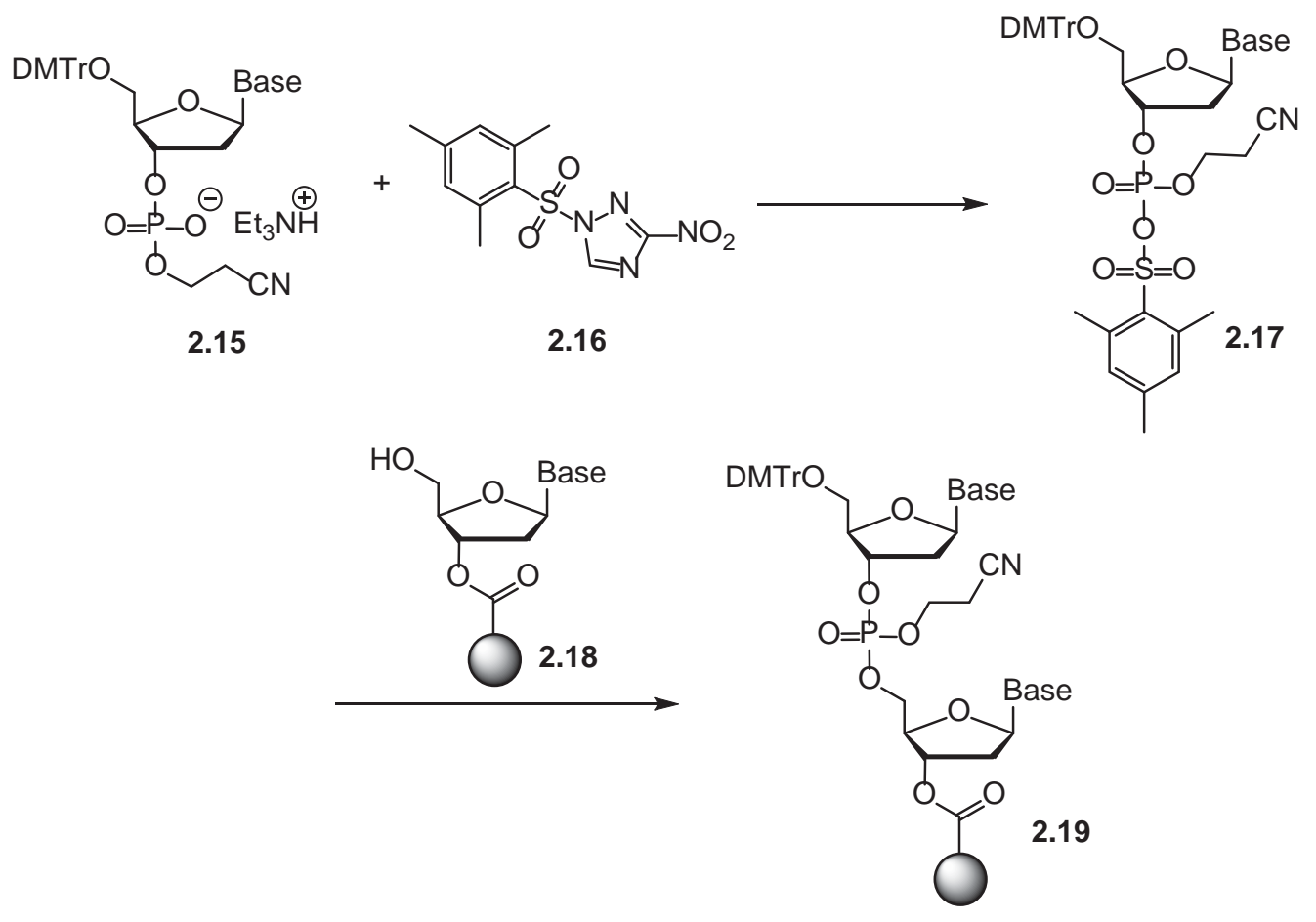

Scheme 2.7. ODN synthesis using phosphotriester method 
The mechanism of the synthesis is shown in Scheme 2.8. The phosphate group of 2.15 is reacted with $\mathbf{2 . 1 6}$ to get mixed anhydride $\mathbf{2 . 1 7}$. Then, $5^{\prime}$-hydroxyl group of $\mathbf{2 . 1 8}$ is coupled with 2.17 to obtain 2.19 as a product.

However, the major problem of this method was the coupling time. The coupling reaction acquired more than one and a half hour to complete. The other drawback is that the coupling is inefficient. It was reported that the average step-wise yield was low from the coupling step. Therefore, the method could not be used to synthesize ODN longer than 20-mer. 

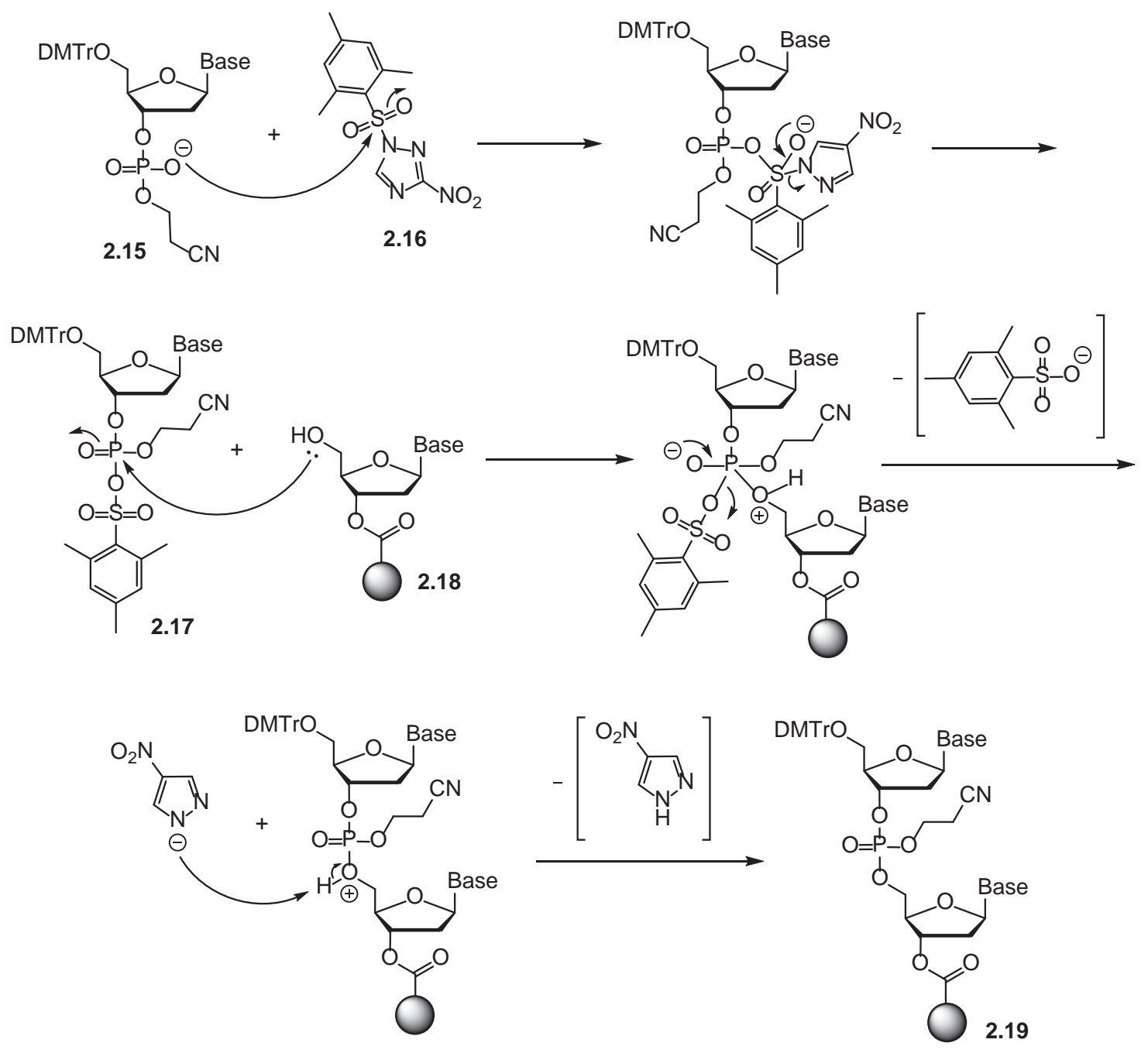

Scheme 2.8. Mechanism of ODN synthesis using phosphotriester method 


\subsection{H-Phosphonate Method}

Todd and Michelson's work on the first ODN synthesis motivated many researchers. Later, a new ODN synthetic method had been developed by two other research groups (Roger Stromberg's group and Mark Matteucci's group). ${ }^{16}$ This method used the $H$ Phosphonate chemistry. As shown in scheme 2.9, 3'- $H$-phosphonate-2'-deoxynucleoside (2.20) and 2.18 were used as starting materials to form the phosphonate interdeoxynucleotide linkage in 2.22. Pivaloyl chloride is a coupling agent to form phosphonate bond. The mechanism of the synthesis was presented in scheme 2.10.

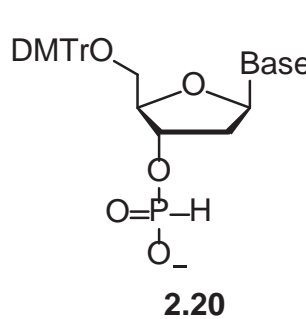

2.20

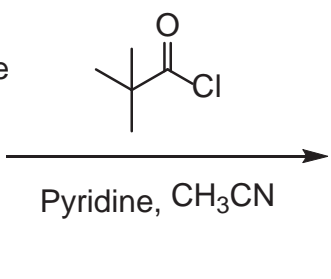

DMTrO

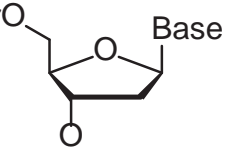

$\mathrm{O}=\mathrm{P}-\mathrm{H}$

○
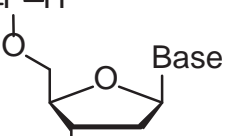

Y

2.22

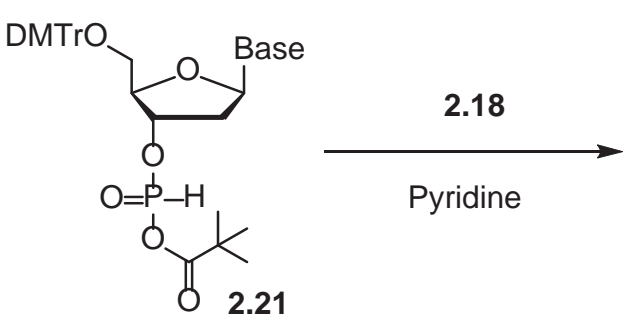

$\mathrm{I}_{2} / \mathrm{H}_{2} \mathrm{O}$

pyridine

DMTrO

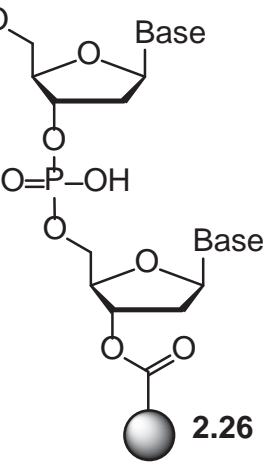

Scheme 2.9. ODN synthesis using $H$-phosphonate method 
According to the Scheme 2.10, the compound 2.20 was acylated with pivaloyl chloride to get the intermediate $\mathbf{2 . 2 1}$ in the form of mixed phosphonic carboxylic anhydride. After that, 5'-hydroxyl group of $\mathbf{2 . 1 8}$ was coupled with $\mathbf{2 . 2 1}$ to obtain the phosphonate 2.22. Then, $H$-phosphonate $\mathbf{2 . 2 2}$ in the form of phosphorus (V) is converted to reactive hydroxyphosphite $\mathbf{2 . 2 3}$ in the form of phosphorus (III) via tautomerization. Next, the lone-pair electrons of phosphorus react with iodine to get iodophosphonium 2.24. Proton absorption using pyridine retrieves iodophosphate 2.25. Finally, hydrolysis of $\mathbf{2 . 2 5}$ in the presence of water and pyridine gives deoxynucleotide $\mathbf{2 . 2 6}$.

This approach has a major problem. $H$-phosphonate activation can cause side reactions. ${ }^{17}$ For example, the reaction requires two to five equivalents of pivaloyl chloride to achieve the high yield of product. The excess amount of pivaloyl chloride can cause acylation of phosphonate $\mathbf{2 . 2 2}$ to get acylphosphonate $\mathbf{2 . 2 7}$ (Scheme 2.11). Also, compound $\mathbf{2 . 2 0}$ can be acylated twice to produce bis-acyl phosphate $\mathbf{2 . 2 8}$. Then, $\mathbf{2 . 2 8}$ can react with two equivalents of $\mathbf{2 . 1 8}$ to form phosphitetriester $\mathbf{2 . 2 9}$ (Scheme 2.12). That will lower yield of the product because their side products cannot convert to get ODN 2.26. 


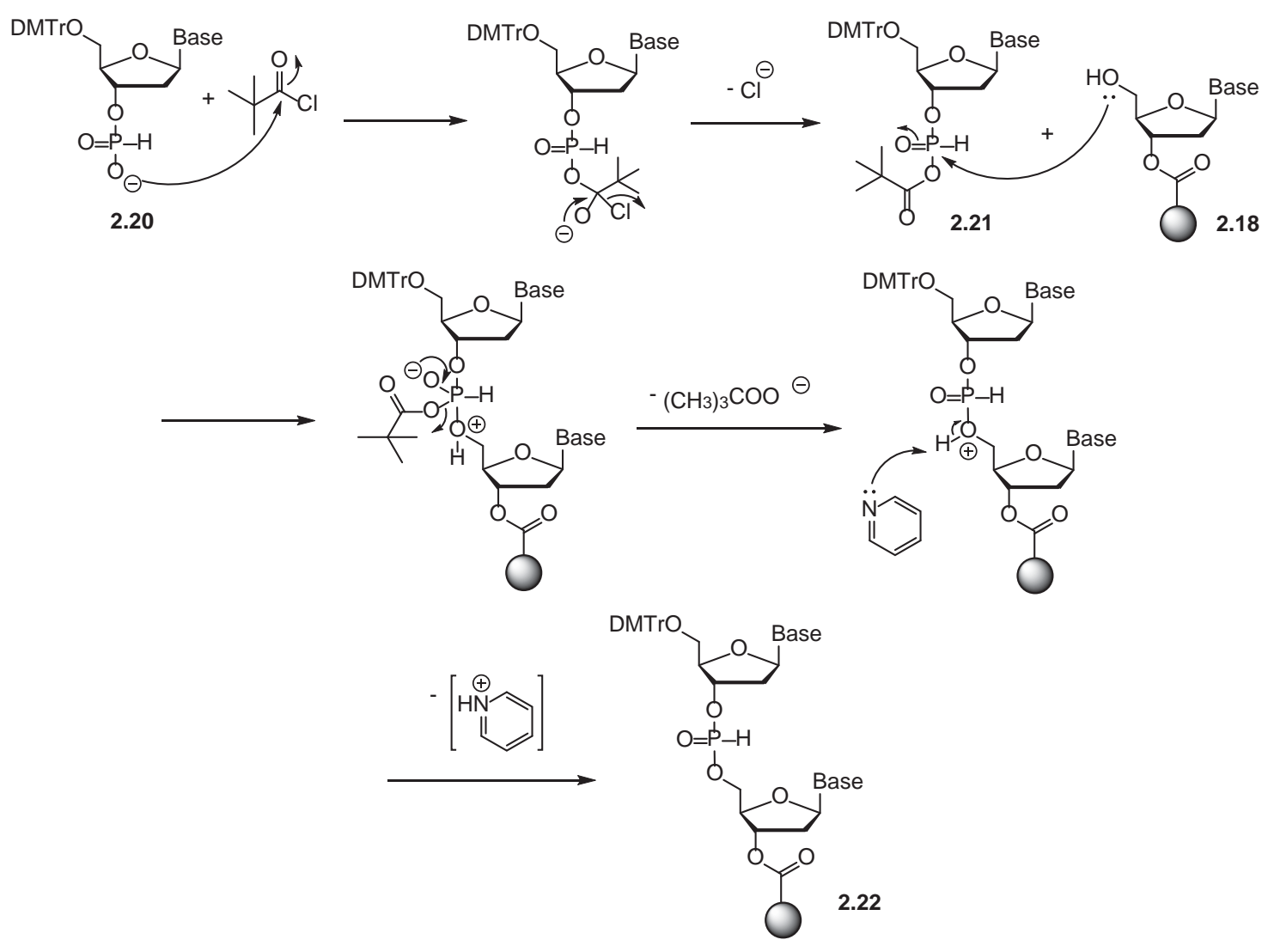

Scheme 2.10. Mechanism of ODN synthesis using $H$-phosphonate method 

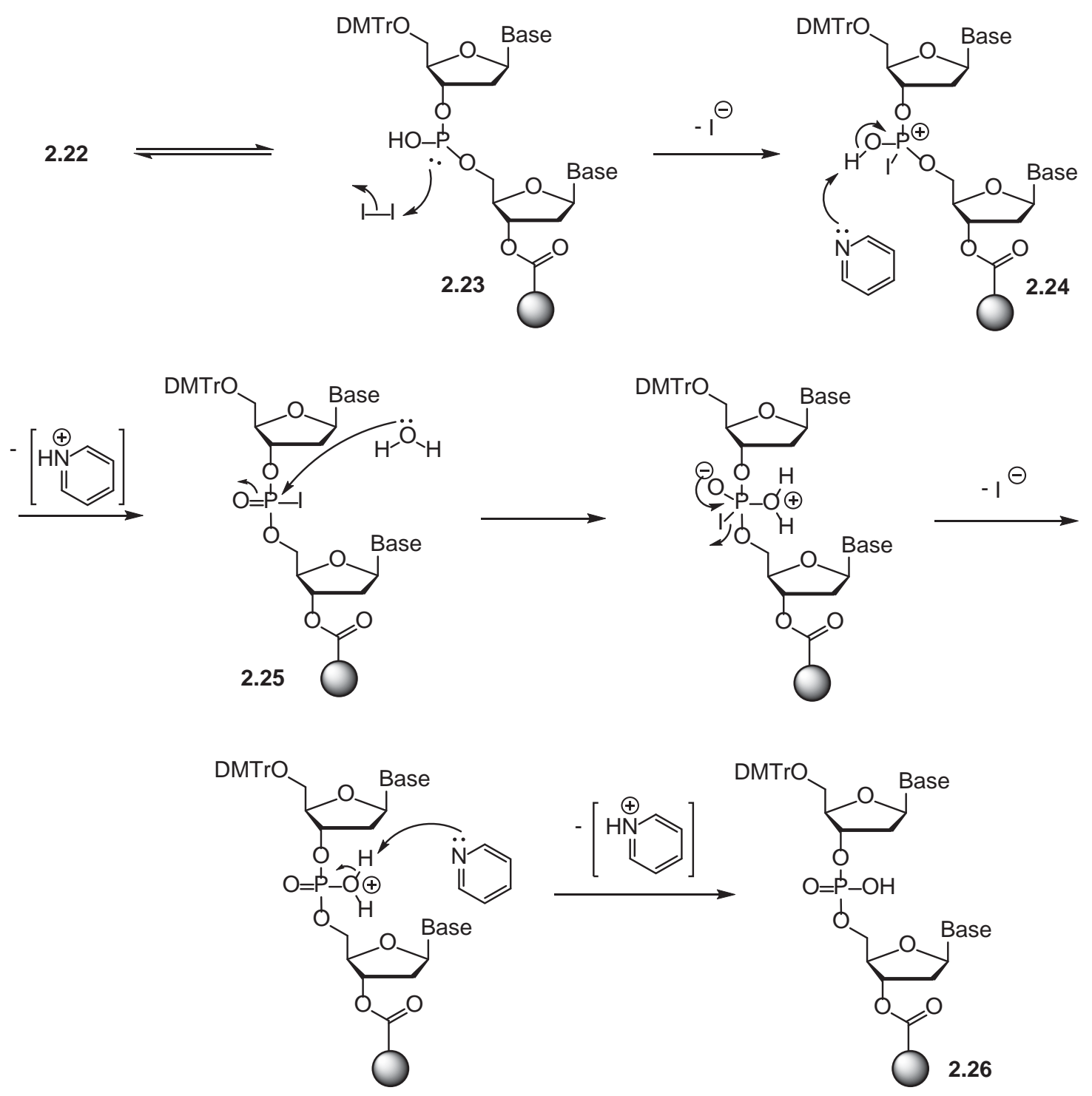

Scheme 2.10 (Continued). Mechanism of ODN synthesis using $H$-phosphonate method 


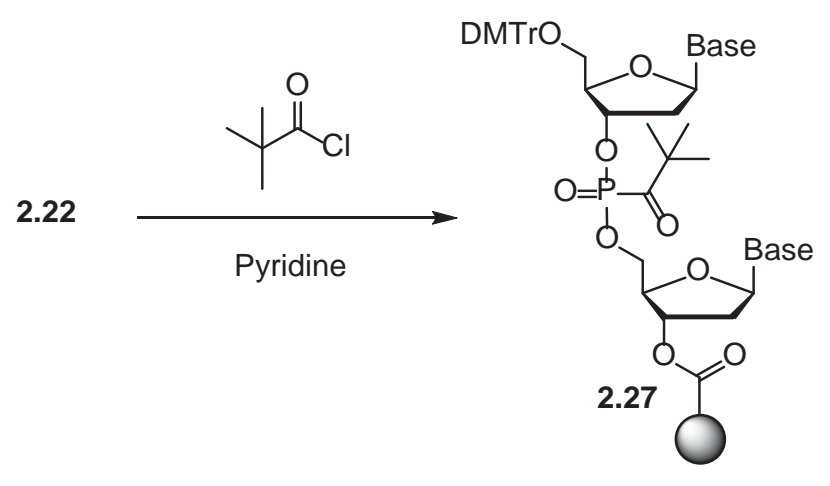

Scheme 2.11. Phosphonate acylation (side reaction) of $H$-phosphonate compound

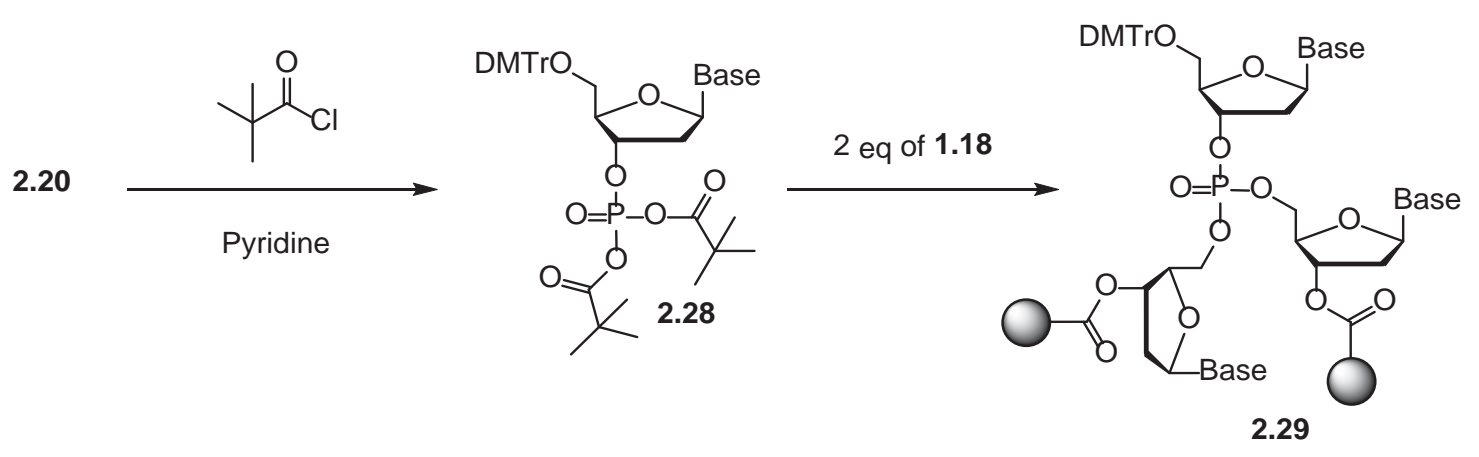

Scheme 2.12. Double acylation (side reaction) of $H$-phosphonate group 


\subsubsection{Phosphite-triester Method}

Since previous methods were suffering from side reactions and long coupling time, researcher tried to find ODN synthesis methods that are more effective than the former methods. In 1975, Letsinger and co-workers published the new ODN synthesis method called phosphite-triester method (Scheme 2.13). ${ }^{18}$ The concept of this reaction is using phosphorus (III) intermediate for coupling instead of phosphorus (V) intermediate. This is because phosphorus (III) compound has much higher reactivity than phosphorus (V) species. Therefore, the reaction time can be significantly reduced and the synthesis can be achieved in higher yields.

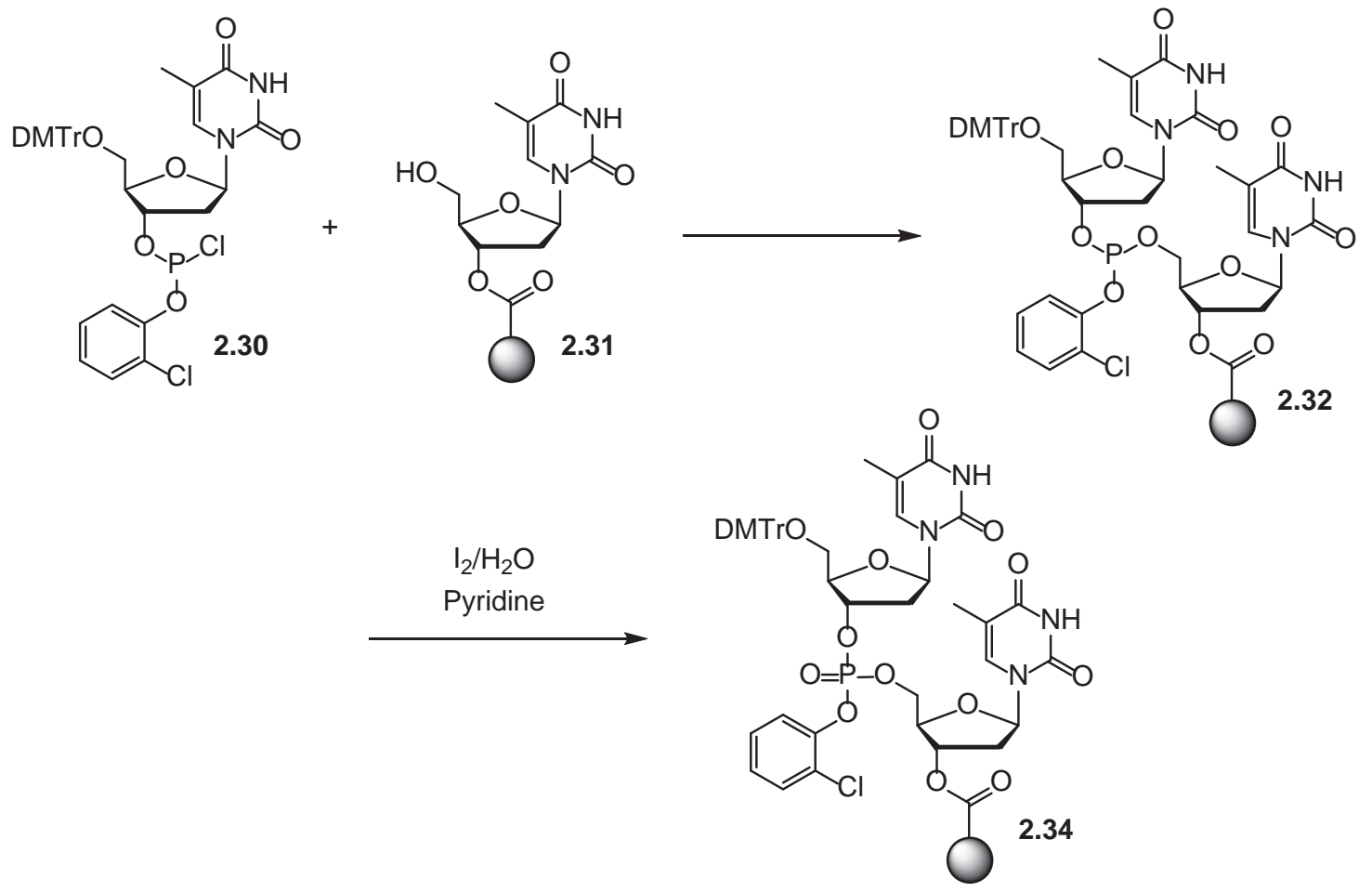

Scheme 2.13. ODN synthesis using phosphite-triester approach 
As illustrated in Scheme 2.14, 5'-hydroxyl group of 2.31 was reacted with phosphomonochloridite 2.30 via bimolecular nucleophilic substitution $\left(\mathrm{S}_{\mathrm{N}} 2\right)$. Then, pyridine absorbed proton to give $\mathbf{2 . 3 2}$. The phosphorus of intermediate $\mathbf{2 . 3 2}$ is oxidized by iodine to get iodophosphonium 2.33. Then, water is used to hydrolyze 2.33 in the presence of pyridine to give phosphate trimester $\mathbf{2 . 3 4}$ as a product.

The major problem of this method is that the phosphomonochloridite is very sensitive to air. The lone-paired electrons on phosphorus can quickly react with oxygen to form inactive phosphate triester. It can also be hydrolyzed by moisture in air. Thus, the intermediate has to be freshly prepared for ODN synthesis. 


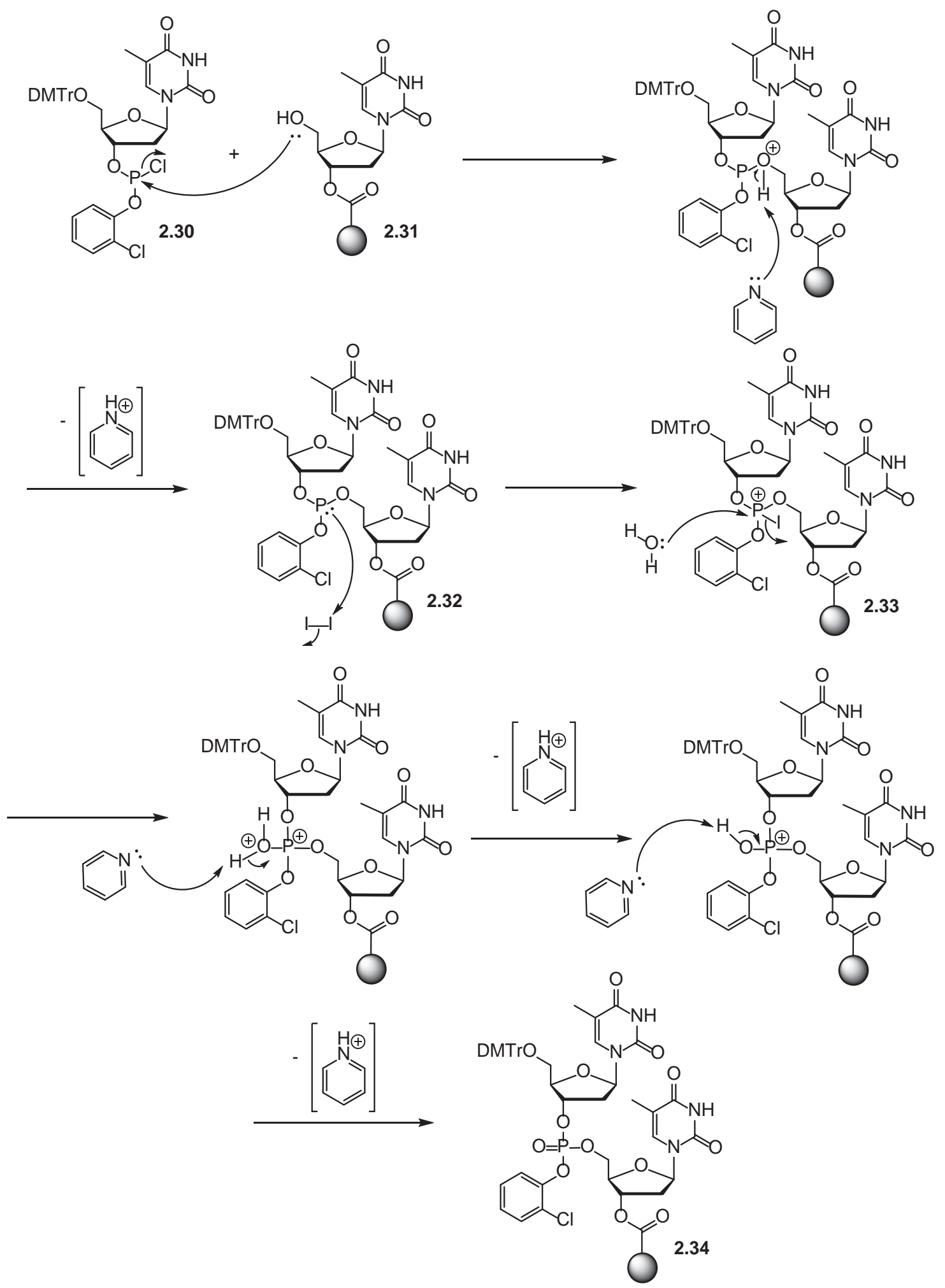

Scheme 2.14. Mechanism of phosphite-triester approach 


\subsubsection{Contemporary ODN synthesis}

By the time of early 1980s, there were two major problems for solid phase ODN synthesis. First, the organic polymer supports (see section 2.3.3) swells in organic solvents. That could block the flow of reagents and solvents when applying to the automated synthesis. Second, the nucleoside intermediates such as phosphonyl nucleoside (see section 2.3.1) and phosphomonochloridite (see section 2.3.6) were not stable. In early 1980s, a new approach of ODN synthesis, which successfully eliminated these problems, had been developed by Caruthers' research group. ${ }^{19}$ The support swelling problem was resolved by using controlled pore glass (CPG). The other problem was overcome by phosphoramidite chemistry. The concept of the reaction resembles phosphite-triester method. However, they changed the leaving group of phosphorus (III) compound from chloride to a diisopropyl amino group. 2-Cyanoethyl (CE) group was used to replace the $o$-chlorophenyl protecting group. This is very magnificent because phosphoramidites are stable in a form of solid. It can be prepared beforehand and stored properly until needed. As a result, this approach is convenient for researchers and it has become widely used for decades. ${ }^{20}$

There are four deoxynucleoside phosphoramidites for ODN synthesis: deoxyadenosine (dA) phosphoramidite, deoxyguanosine (dG) phosphoramidite, deoxycytidine (dC) phosphoramidite, deoxythymidine (dT) phosphoramidite (Figure 2.7). $5^{\prime}$-OH group of each phosphoramidite is also protected with $4,4^{\prime}$-dimethoxytrityl (DMTr) group. The exocyclic amino groups of $\mathrm{dA}, \mathrm{dC}$, and $\mathrm{dG}$ are protected to prevent side reactions during the coupling step. For stanadard ODN synthesis, the amino group of dA is protected by benzoyl $(\mathrm{Bz})$ group. The amino group of $\mathrm{dG}$ is protected by isobutyryl ( $i$ - 
$\mathrm{Bu}$ ) group. The amino group of $\mathrm{dC}$ is protected by acetyl (Ac) group. Because $\mathrm{dT}$ does not have exocyclic amino group, it does not need any protection. For ultramild ODN synthesis, $\mathrm{dC}$ phosphoramidite and $\mathrm{dT}$ phosphoramidite are the same as those used in the standard ODN synthesis. However, the amino groups of dA and dG are protected by phenoxyacetyl (Pac) group and 4-isopropyl-phenoxyacetyl (i-Pr-Pac) group respectively (Figure 2.8).

Later, Caruthers and Hood developed automated system for ODN synthesis. The system combined solid phase synthesis and phosphoramidite chemistry. During collaboration, they formed a company named Applied Biosystems, Incorporated (ABI). They also exploited the first phosphoramidite DNA synthesizer which benefits to ODN research nowadays. The concept of automated synthesis is a synthetic cycle. For each cycle, addition of phosphoramidite takes place at 5'-end of ODN strand. The synthesis continues until the chosen sequence is complete. ODN synthetic cycle is illustrated in Scheme 2.15. It contains four steps that are described in the next section. 


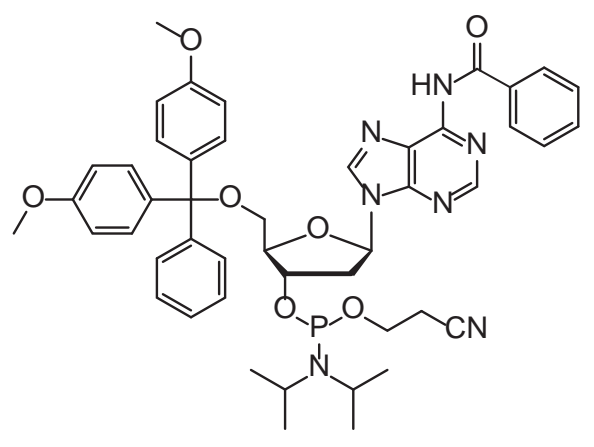

Bz-dA-CE phosphoramidite

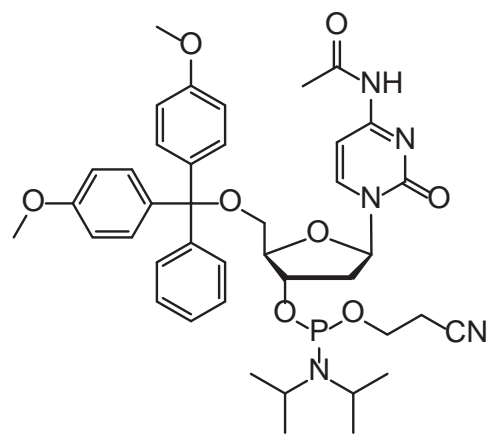

Ac-dC-CE phosphoramidite

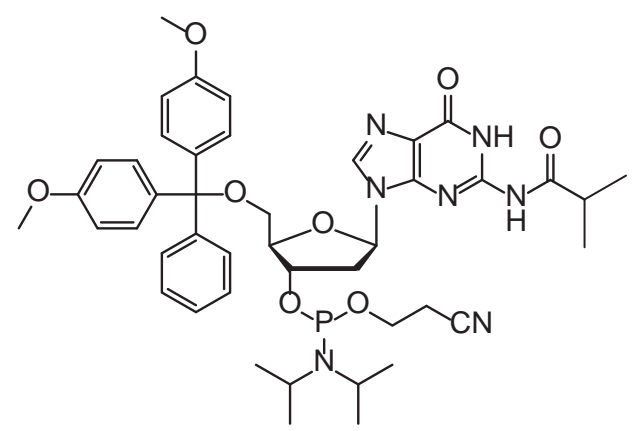

i-Bu-dG-CE phosphoramidite

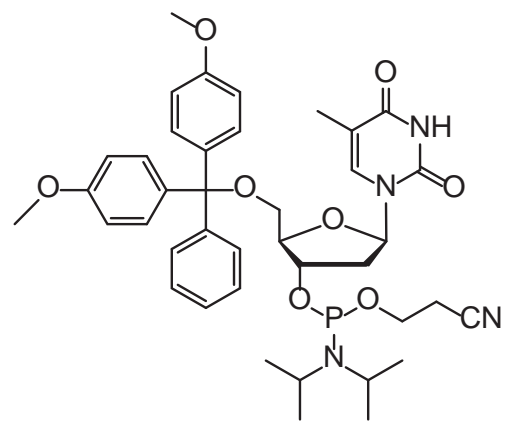

dT-CE phosphoramidite

Figure 2.7. Deoxynucleoside phosphoramidites

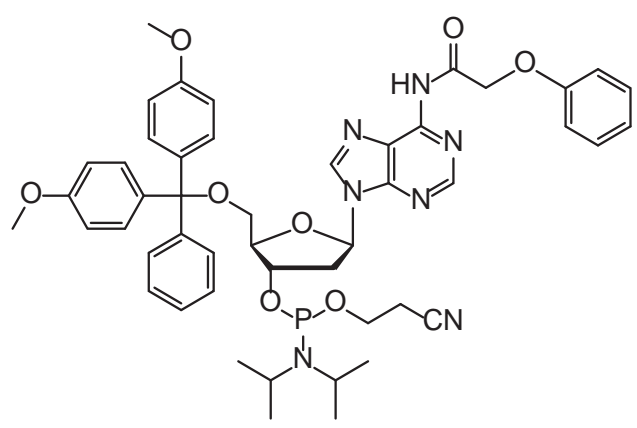

Pac-dA-CE phosphoramidite

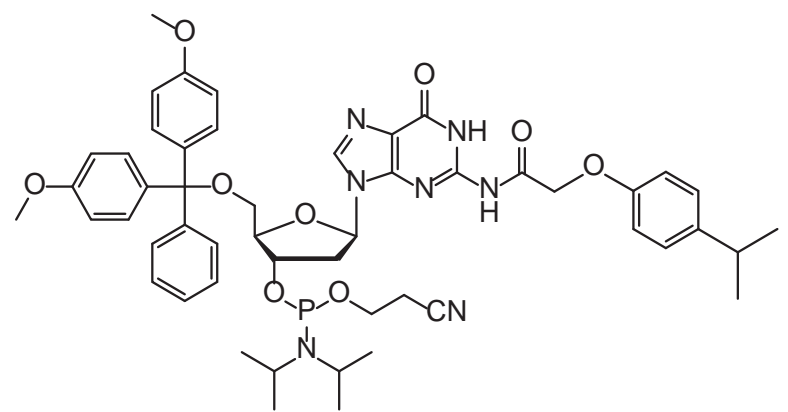

IPr-Pac-dG-CE phosphoramidite

Figure 2.8. Pac-dA-CE phosphoramidite and $i$ Pr-Pac-dG-CE phosphoramidte for Ultramild ODN synthesis 


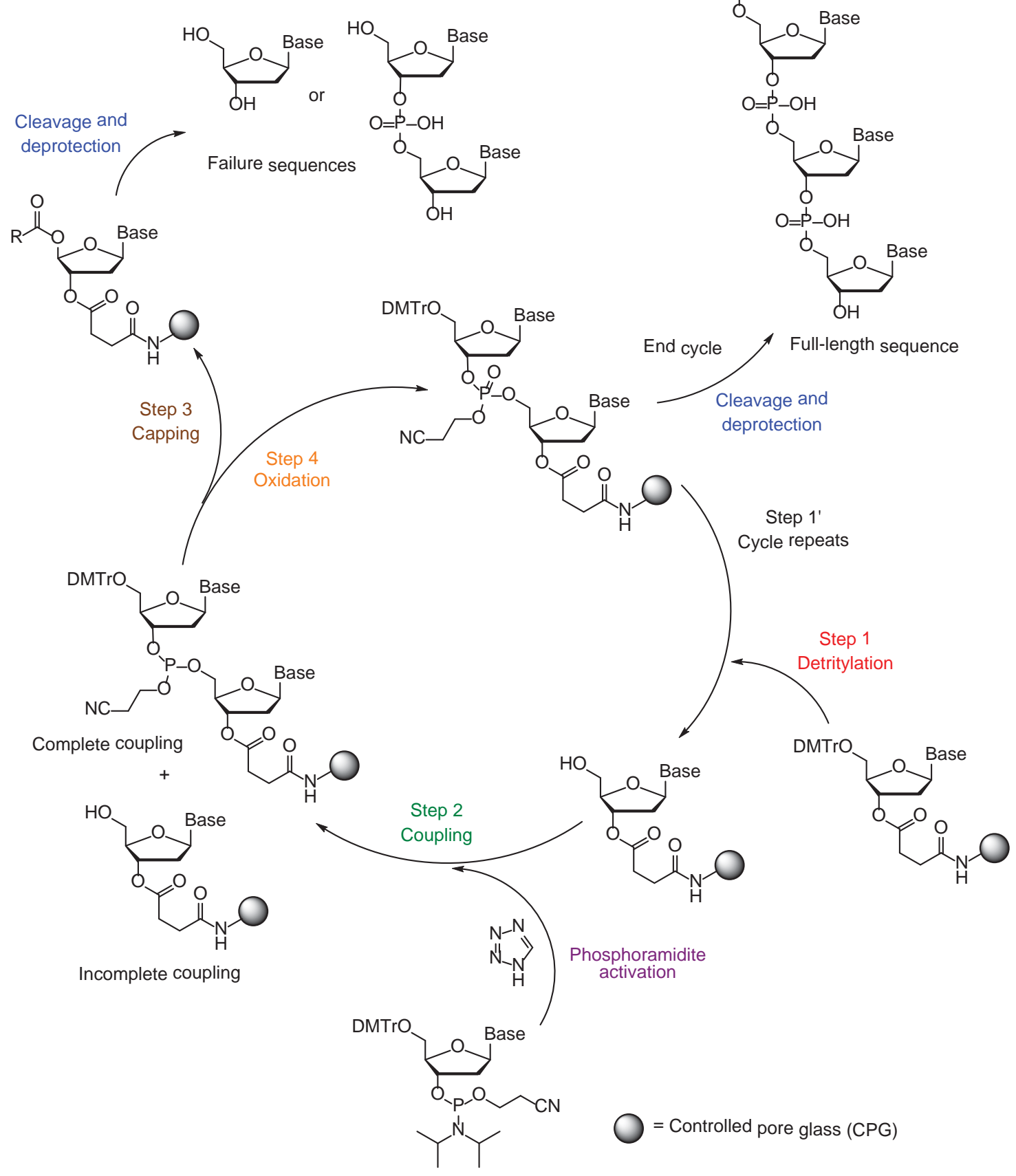

Scheme 2.15. ODN synthesis using phosphoramidite approach 


\subsubsection{ODN synthetic cycle}

\subsubsection{Detritylation}

4,4'-Dimethoxytrityl (DMTr) group, which protects 5'-hydroxyl group of the initial deoxynucleoside, is removed in this step. Therefore, the incoming phosphoramidite monomer can couple at $5^{\prime}$ end. DMTr group is acid-labile enough that can be cleaved using acidic solution (2-3 \%), such as dichloroacetic acid or trichloroacetic acid in dichloromethane. The mechanism of detritylation is shown in Scheme 2.16. Oxygen atom at $5^{\prime}$ position of $\mathbf{2 . 3 5}$ is protonated by the acid. Then, orange-colored DMTr cation (2.37) is released from the CPG-linked nucleoside to get 2.36. The cation is stable because it can form several resonance structures (Scheme 2.17).

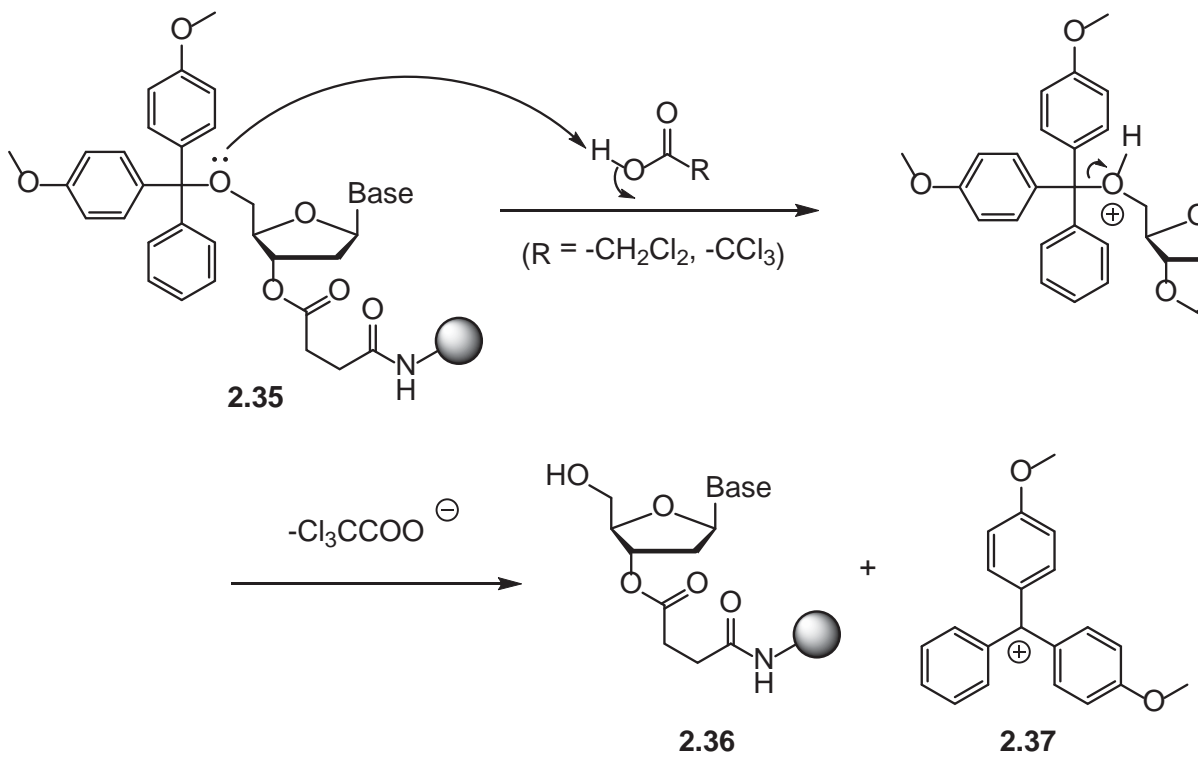

Scheme 2.16. Mechanism of detritylation 


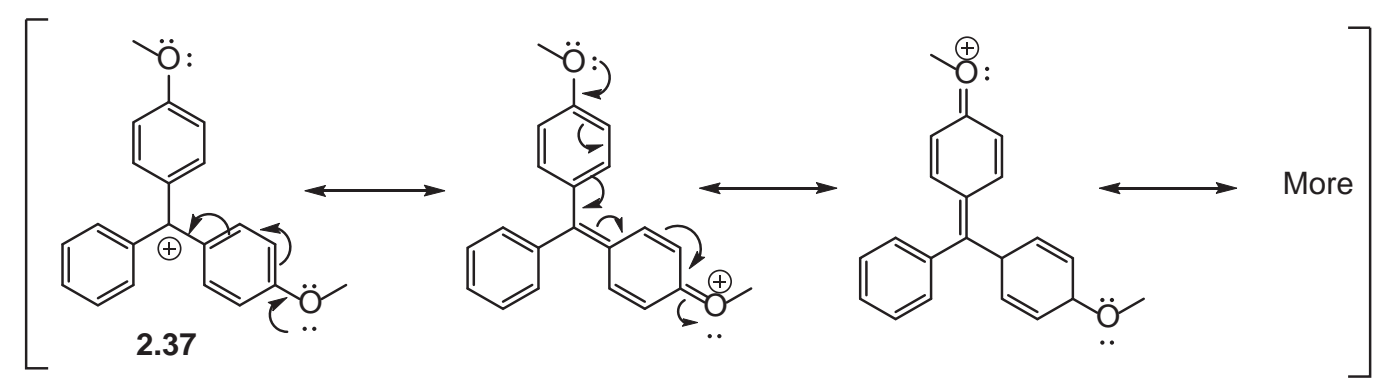

Scheme 2.17. Resonance structures of DMTr cation

\subsubsection{Coupling}

When detritylation is finished, the solution of phosphoramidite monomer is delivered. The phosphoramidite is activated by a weak acid catalyst such as $1 H$-tetrazole, 4,5-dicyanoimidazole (DCI), etc. Then, the activated phosphoramidite is coupled at 5'hydroxyl group of CPG-linked nucleoside. The most common weak acid used for activation is $1 H$-tetrazole. The $\mathrm{pK}_{\mathrm{a}}$ of $1 H$-tetrazole is 4.9 , which is adequately acidic to activate phosphoramidite but not acidic enough to deprotect DMTr group. However, some researchers considered $1 H$-tetrazole as an explosive compound. ${ }^{21}$ In addition, there were reports that $1 H$-tetrazole could cause premature detritylation, which results addition sequences, so replacing it with the other activating agent, such as 4,5-dicyanoimidazole (DCI) was pursued. ${ }^{22}$ Although DCI is less acidic than $1 H$-tetrazole $\left(\mathrm{pK}_{\mathrm{a}}=5.2\right)$, it can activate phosphoramidite efficiently. It is also less harmful than $1 H$-tetrazole for largescale ODN synthesis.

The mechanism of phosphoramidite activation and coupling is proposed in Scheme 2.18, the diisopropylamino group in $\mathbf{2 . 3 8}$ is protonated by $1 H$-tetrazole to generate a good leaving group of diisopropylammonium. Next, tetrazole anion is acted as a nucleophile to displace the diisopropylammonium to get $\mathbf{2 . 3 9}$ and diisopropylamine. Then, 
5' hydroxyl group of $\mathbf{2 . 3 6}$ attacks to the P(III) atom of $\mathbf{2 . 3 9}$ to form phosphite-triester $\mathbf{2 . 4 0}$. In the final step, the proton is absorbed by diisopropylamine to form $\mathbf{2 . 4 1}$ as a side product.

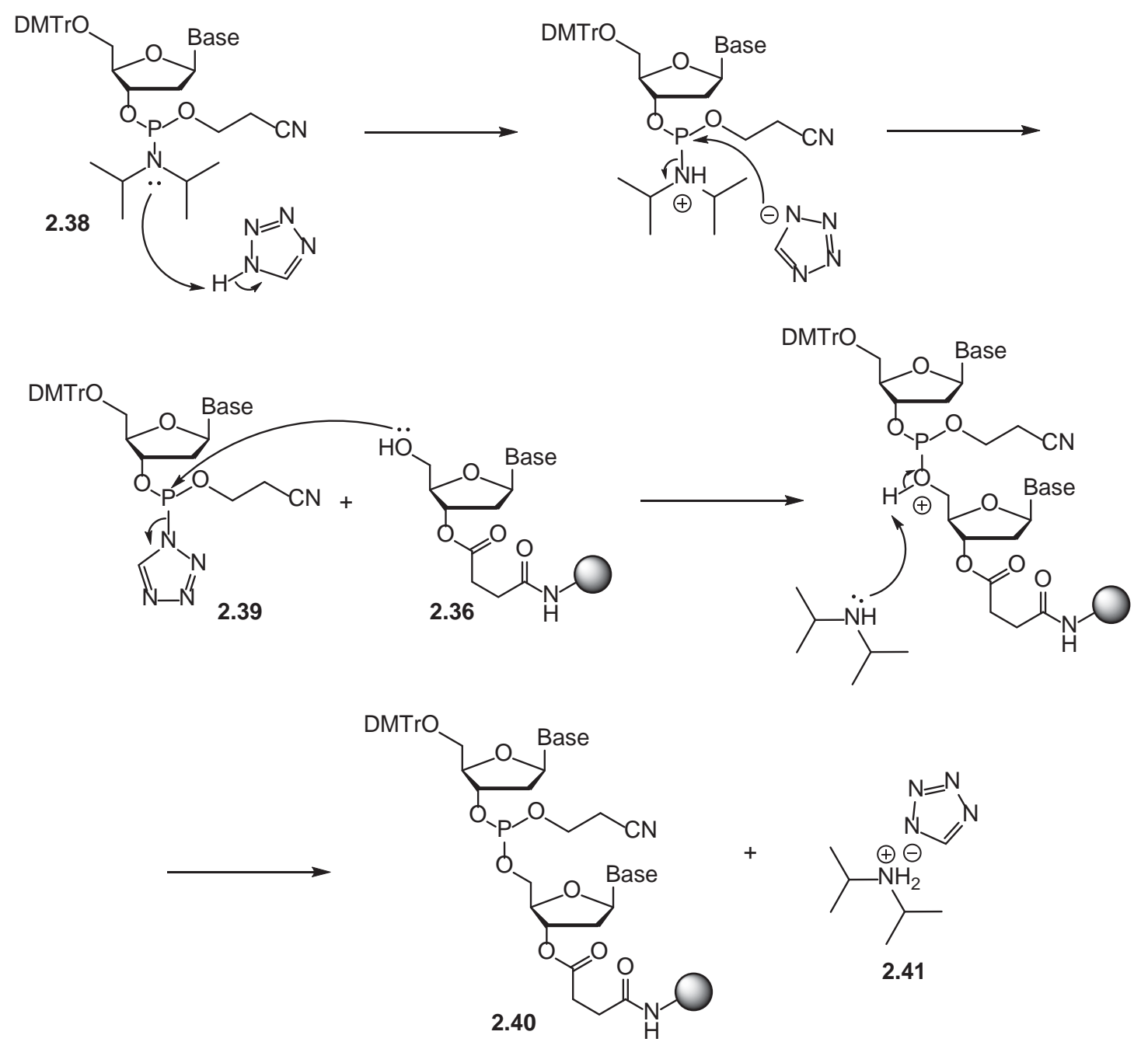

Scheme 2.18. Mechanism of phosphoramidite activation and coupling 


\subsubsection{Capping}

In spite of rapid coupling of phosphoramidite, it is inevitable that the reaction cannot be $100 \%$ complete. There are a few unreacted 5'-unreacted hydroxyl groups, which result from failed coupling. These can be available to react with the phosphoramidite monomer in the next cycle. This will generate deletion sequences that miss one nucleotide (Figure 2.9). If the ODN synthesis is left unchecked, deletion sequences accumulate from each synthetic cycle. The final ODN crude product would contain a complexed mixture of full-length sequence and deletion sequences. That would make purification very challenging.
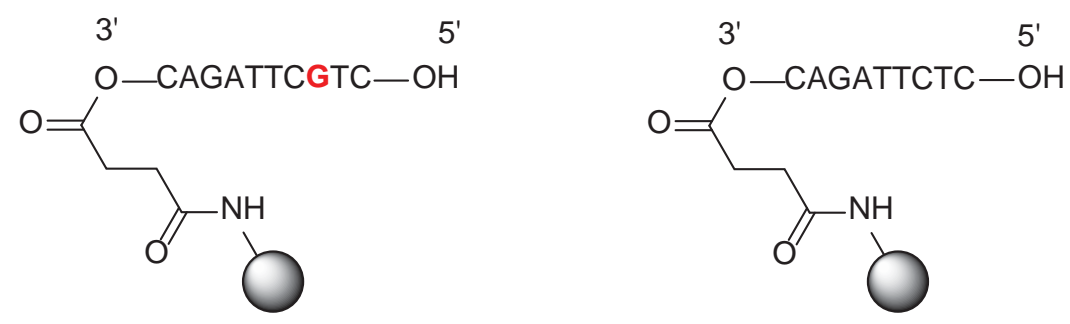

Figure 2.9. Example of deletion sequence (right). The $\mathrm{dG}$ at $8^{\text {th }}$ position was missing compared to the full-length sequence (left)

To solve the problem, 5'-unreacted hydroxyl groups are blocked using a capping agent to terminate the failure sequences. There are two standard capping solutions on DNA synthesizer. One is the mixture of acetic anhydride and small amount of pyridine in THF. The other is $\mathrm{N}$-methylimidazole (NMI) in THF. For Ultramild synthesis, phenoxyacetic anhydride is used instead of acetic anhydride because it avoids undesired side reactions. During capping, these two solutions are delivered to the synthesis column at the same time. 
The mechanism is shown in Scheme 2.19. 1-Methylimidazole is reacted with acid anhydride to get intermediate $\mathbf{2 . 4 2}$. Then, unreacted 5'-hydroxyl group of $\mathbf{2 . 3 6}$ is acylated with $\mathbf{2 . 4 2}$ to get capped ODN 2.43.

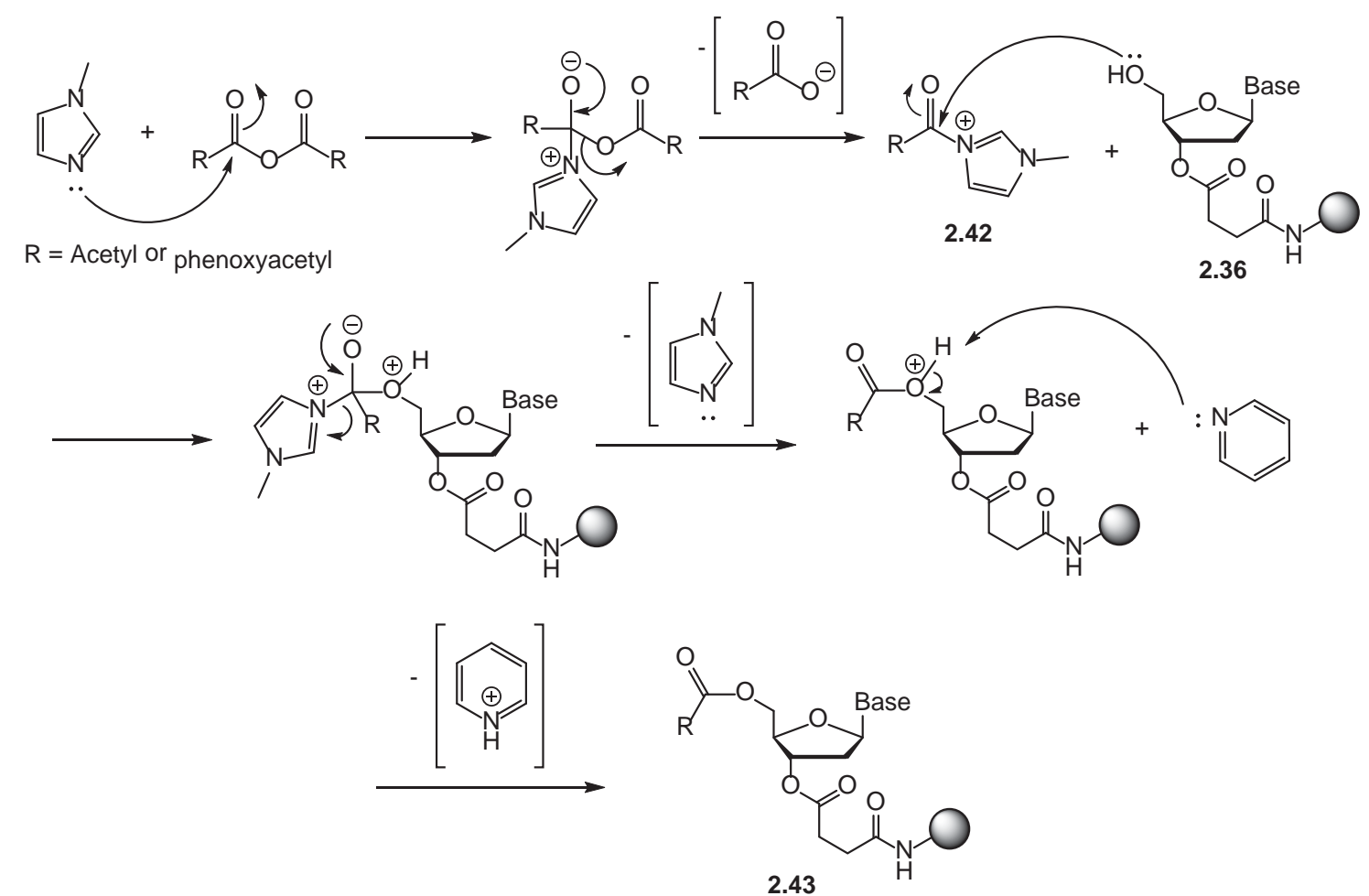

Scheme 2.19. Mechanism of capping 


\subsubsection{Oxidation}

The newly formed phosphite-triester bond, which contains phosphorus (III), is not stable to acid. So, it has to be converted to stable phosphorus (V) species by oxidation before detritylation of the next cycle. The standard oxidizing agent, which is commonly used, consists of iodine $\left(\mathrm{I}_{2}\right)$, water, and organic weak base (pyridine, 2,6-lutidine, or collidine) in THF. The mechanism is shown in Scheme 2.20. Phosphite-triester 2.40 is transformed to iodophosphonium $\mathbf{2 . 4 4}$ by iodine. Then, hydrolysis of 2.44 using water and proton removal using pyridine gives product $\mathbf{2 . 4 5}$. Since the next coupling reaction could be inhibited by water residue from the oxidizing agent, some researchers prefer to carry out the capping step after oxidation. Thereby, the solid support could be more likely to be dry before the next cycle.

Alternative oxidizing agents have been studied. For example, some researchers had developed non-aqueous oxidizing agents for anhydrous conditions. Examples include tertbutyl hydroperoxide $\left(t\right.$-BuOOH), cumene hydroperoxide, bis-trimethylsilyl peroxide, etc. ${ }^{23}$ However, these are not widely used because they are not stable. All of them must be

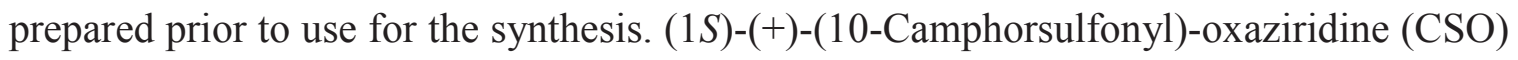
is the other commercially available non-aqueous oxidizer. It is very useful for synthesis of certain special ODNs which can be degraded by iodine. Examples include DNAphosphonoacetate, ${ }^{24}$ and ODN containing 7-deaza-deoxyguanosine. ${ }^{25}$ 


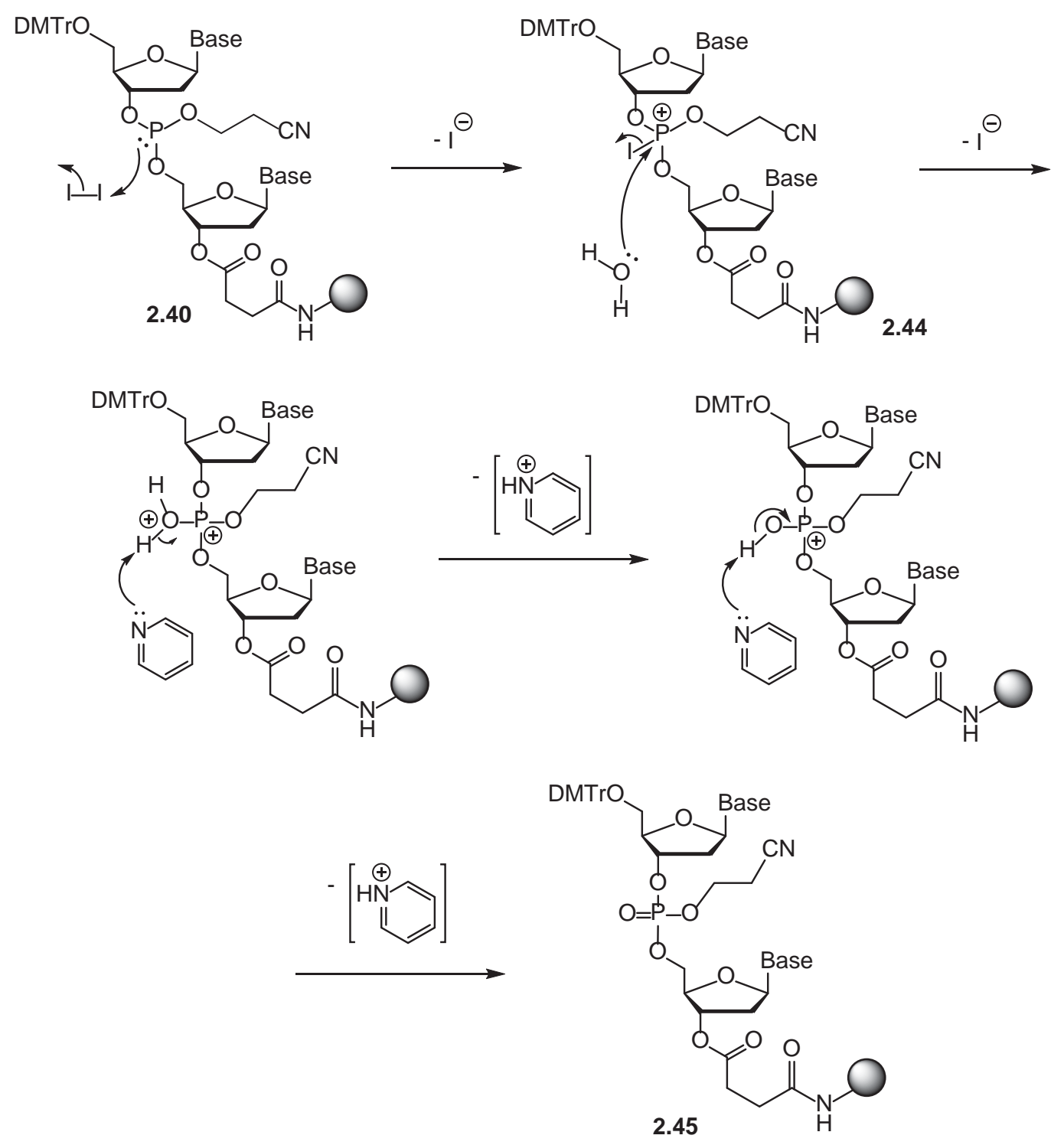

Scheme 2.20. Mechanism of oxidation 


\subsubsection{Cleavage and Deprotection}

The previous four steps are repeated as one cycle for adding each nucleoside phosphoramidite monomer until the ODN synthesis is complete. After that, ODN on the solid support, which is attached to CPG, is released from the solid support by linker cleavage. Also, all of the protecting groups on the nucleobases and phosphates are deprotected. These are achieved using concentrated ammonium hydroxide $\left(\mathrm{NH}_{4} \mathrm{OH}, 28 \%\right)$ solution. Ammonium hydroxide is the most widely used reagent for this step because it can cleave and deprotect ODN simultaneously. As seen in Scheme 2.21, mechanisms are described in the followings:

\subsubsection{Cleavage ODN from solid support}

The succinyl ester linker, which connects 3 '-end of synthetic ODN to CPG, is readily cleaved by aminolysis. In the Scheme, ammonia acts as a nucleophile to react the carbonyl group of succinyl ester. Then, the ODN is cleaved from the CPG. For automated synthesis, ammonium hydroxide solution is usually delivered to the CPG for four times. Each time, cleavage reaction is allowed to proceed for 15 minutes. Argon was used to flush the ODN solution to a glass vial. Manual cleavage of ODN can also be achieved by shaking the CPG with ammonium hydroxide solution. 


\subsubsection{Phosphate deprotection}

The 2-cyanoethyl groups, which protect phosphate groups during the entire synthesis are removed. As shown in Scheme 2.21, a proton on the $\alpha$-carbon next to cyanogroup is quickly deprotonated by ammonia. Then, $\beta$-elimination gives ODN in a form of ammonium phosphate and acrylonitrile as a by-product. In addition, 1,8diazabicyclo[5.4.0]undec-7-ene (DBU), which is a non-nucleophilic base, can also be used for deprotecting 2-cyanoethyl groups.

\subsubsection{Nucleobase deprotection}

Protecting groups on nucleobases are deprotected by simple amide aminolysis. For normal conditions, the nucleobase with standard protecting group is treated in ammonium hydroxide solution at $55^{\circ} \mathrm{C}$ for 8 to 15 hours. For Ultramild conditions, protecting groups can be removed in the same solution but room temperature for 1 to 2 hours. 

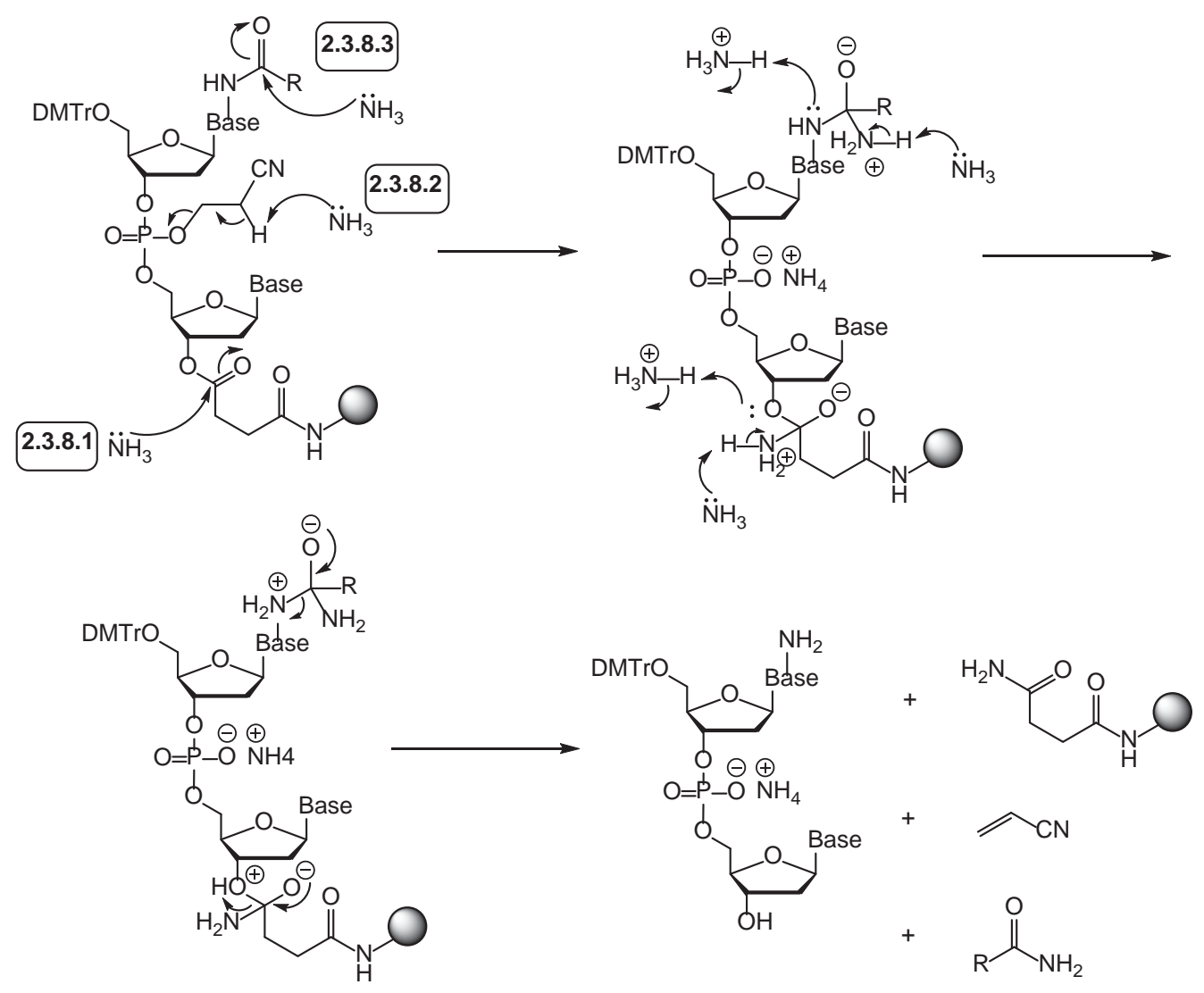

$\mathrm{R}=$ acetyl, benzoyl, isobutyryl, phenoxy acetyl, or 4-isopropyl-phenoxyacetyl

Scheme 2.21. Mechanism of cleavage, phosphate deprotection, and nucleobase deprotection 


\subsubsection{Quenching of by-product}

Acrylonitrile, which is generated from phosphate deprotection, is a Michael acceptor. It is reactive enough to react with nucleobases through Michael addition at room temperature. That causes undesired and modified ODN. As illustrated in Scheme 2.22, amino group of adenine, cytidine, and guanine can react with acrylonitrile to get 2cyanoethyl adduct. Michael addition of thymine needs ammonia to deprotonate the proton at N-3 position of thymine. Then, the nucleophile reacts acrylonitrile to get the thymine adduct (Scheme 2.23).
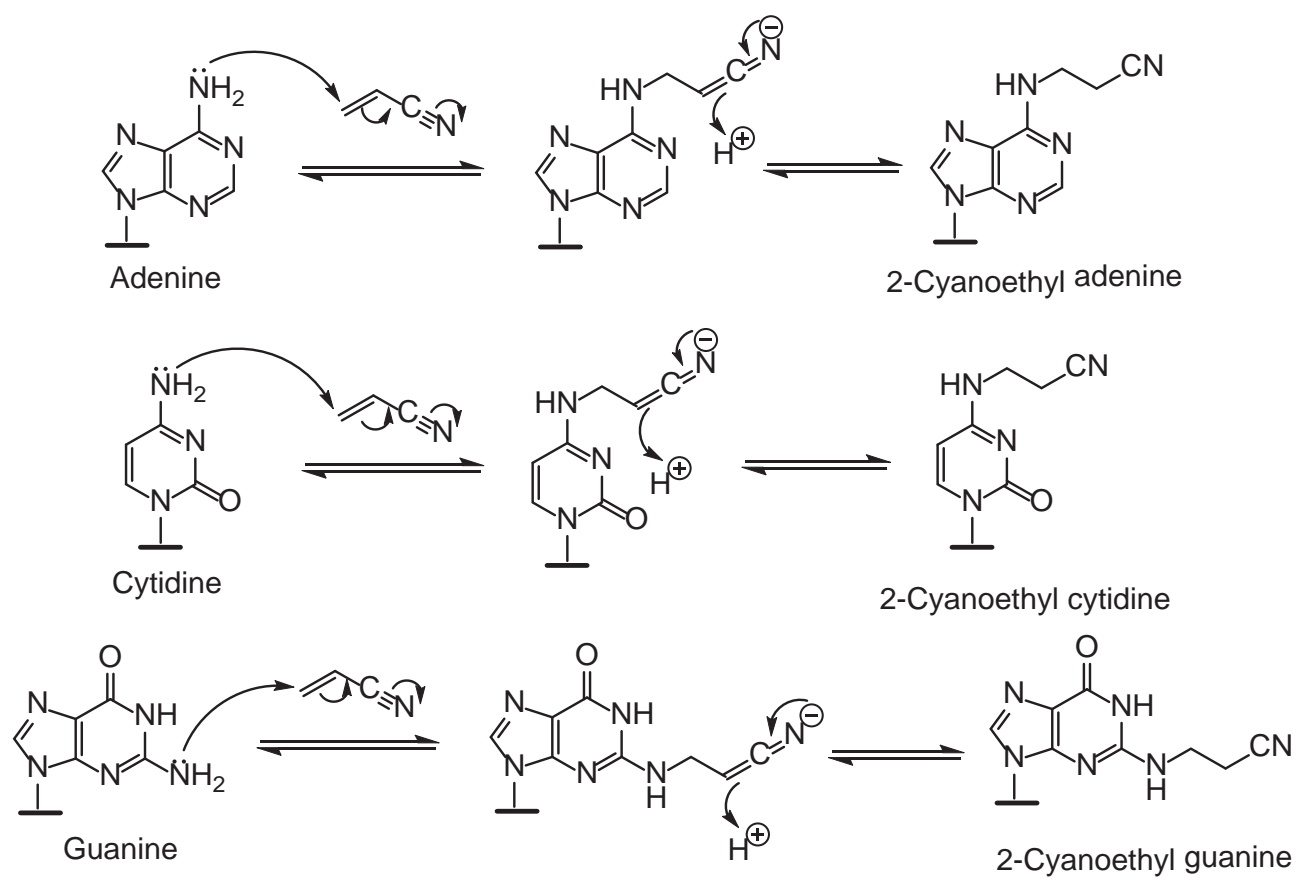

Scheme 2.22. Michael addition of adenine, cytidine, and guanine with acrylonitrile 

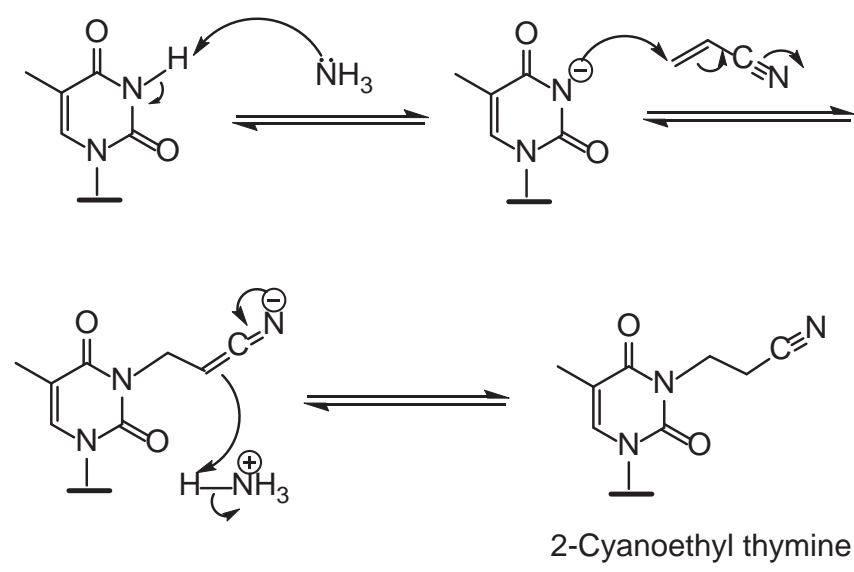

Scheme 2.23. Michael addition of acrylonitrile to thymine

Since Michael addition is a reversible reaction, un-modified nucleobases can be retrieved by $\mathrm{NH}_{4} \mathrm{OH}$. Excess ammonia will deprotonate the proton on the carbon which is next to the electron withdrawing cyano-group. Then, $\beta$-elimination would give unmodified nucleobases (Scheme 2.24). The excess ammonia also scavenges the remaining acrylonitrile to give 3-aminopropanenitrile which is no longer Michael acceptor (Scheme 2.25).

Some researchers adjusted the method to avoid Michael adducts. This was achieved by completing phosphate deprotection before cleavage and nucleobase deprotection. CPGbound ODN was treated with an organic weak base in an organic solvent, such as $10 \%$ diethylamine in acetonitrile, $10 \% \mathrm{DBU}$ in acetonitrile, or 1:1 triethylamine/acetonitrile. Phosphate groups are deprotected while ODN remains on the CPG. Acrylonitrile cannot react any nucleobases which are still protected. Thus, it will be washed away by the organic solvent. 

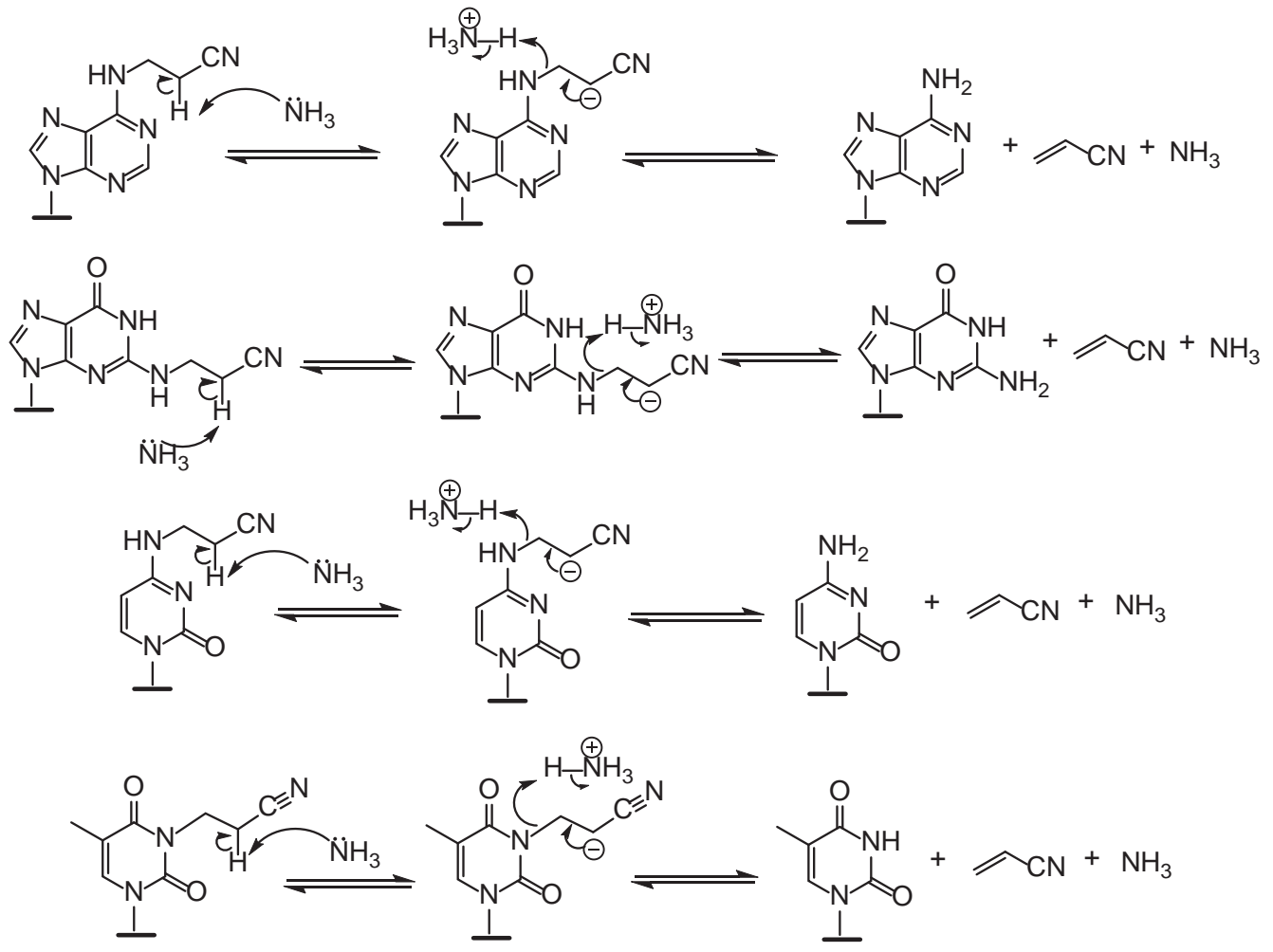

Scheme 2.24. Reversed Michael reaction in excess of ammonia

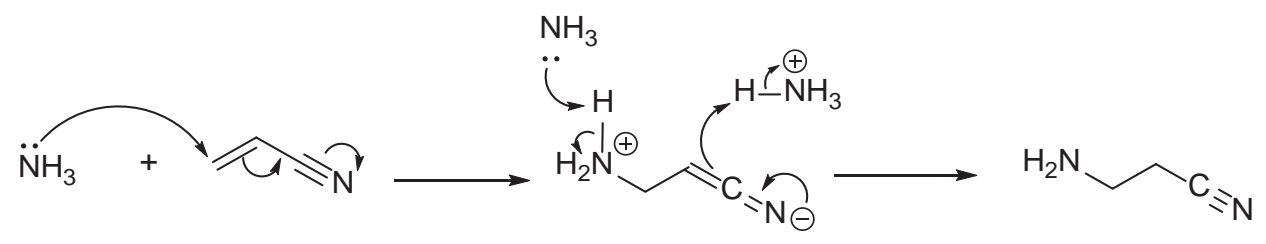

Scheme 2.25. Mechanism of scavenging acrylonitrile in ammonia 


\subsection{Impurities in Crude ODN}

After automated synthesis, final crude ODN contains the mixture of full-length ODN and other impurities. The amount of impurities and the yield of pure ODN depends on quantity of synthesis, quality of solvents and reagents, and cautiousness of operating automated synthesizer. For instance, 20-mer ODN synthesis typically contains $40-70 \%$ of full-length ODN and 30-60 \% of impurities. There are several types of impurities in crude ODN. ${ }^{26}$

\subsubsection{Small Organic Molecules}

Mostly, they are generated from removing the protecting groups. Examples include acetamide, benzylamide, isobutylamide, 3-aminopropanenitrile etc. Since they are neutral molecules, they can be easily removed by ethanol or $n-\mathrm{BuOH}$ precipitation ${ }^{27}$ from an aqueous buffer. ODN, which is negative charged in the buffer, is insoluble in ethanol or $n$ $\mathrm{BuOH}$. Then, ODN is precipitated. The small molecules are soluble and remaining in the supernatant. Besides precipitation, size-exclusion chromatography can be used to remove small organic molecules (See 2.5.1).

\subsubsection{Failure Sequences}

\subsubsection{Usual failure sequences}

They are generated from the coupling step due to incomplete coupling. The longer the strand of ODN is synthesized, the more failure sequences are produced. Those sequences will be capped with a capping agent, such as acetic anhydride, or phonoxyacetic anhydride (For Ultramild conditions) to stop elongation which cause deletion sequences. 
Because the properties of the failure sequences are similar as the full-length ODN, removing failure sequences are challenging.

\subsubsection{Unusual failure sequences}

\section{(1) Deletion sequences}

They can be produced from incomplete capping. The failure sequences without capping still have free 5'-OH groups. These hydroxyl groups can couple with the coming phosphoramidite monomer in the next synthesis cycles. Hence, the sequences will be missing a nucleotide (Scheme 2.26).

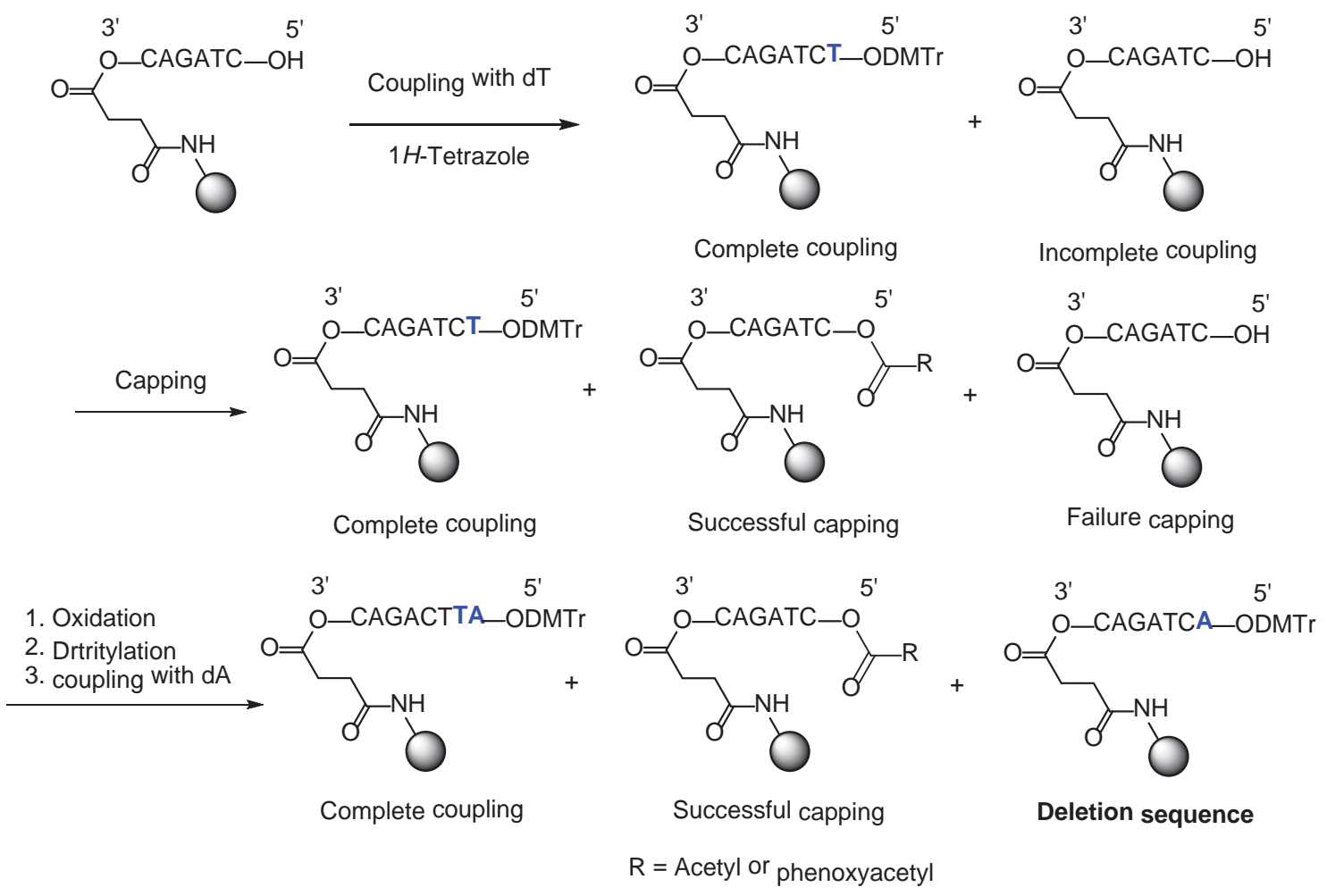

Scheme 2.26. Formation of deletion sequence 
(2) Addition sequences

Addition sequences are the sequences that contain one or more extra nucleobases. They are resulted from premature detritylation. So, the phosphoramidite monomer can be added to the ODN strand twice (Scheme 2.27).

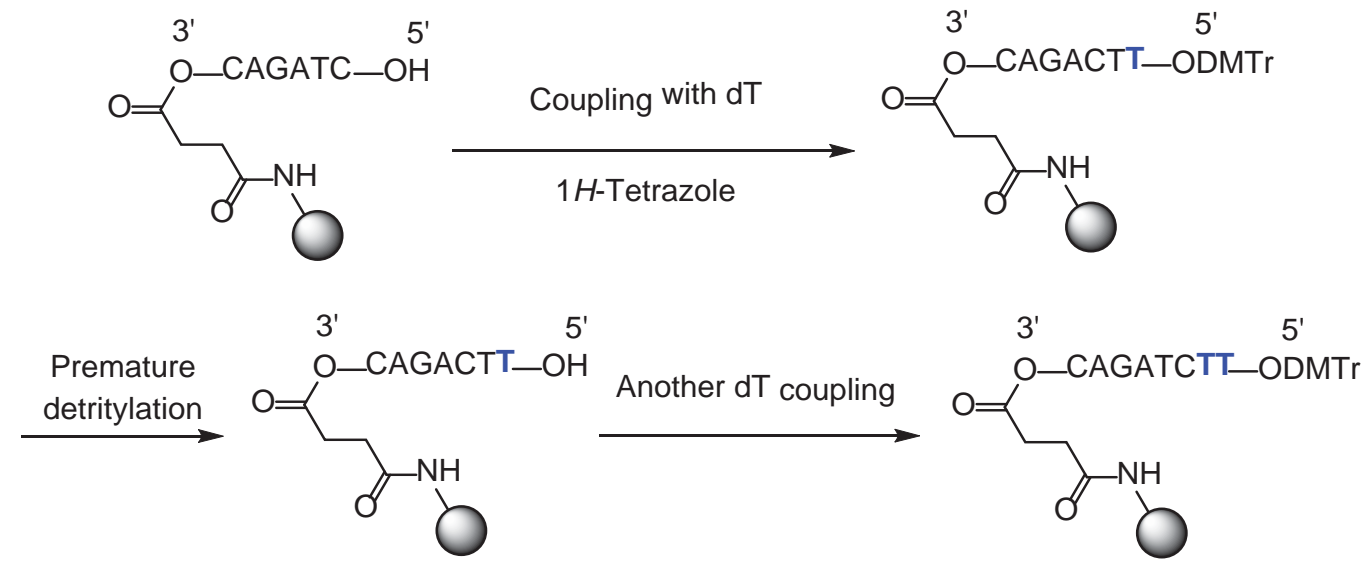

Scheme 2.27. Formation of addition sequence during coupling step

For deletion sequences and addition sequences, they are very difficult to remove. Luckily, they occur in very small amount in crude ODN because the conditions for contemporary ODN synthesis have been designed to prevent this type of impurities. Thus, the main task of ODN purification is focusing on removing failure sequences from the fulllength ODN. 


\subsection{Current Methods for ODN Purification and Limitations}

Purification is a major task for ODN production for many applications. Typically, there are several methods to purify crude ODN.

\subsubsection{Size-exclusion Chromatography}

The principle of size-exclusion chromatography is separation of molecules by size. The packing material in the column contains a lot of pores. When crude ODN solution is loaded, smaller molecules enter deeply in the pores. So, they are eluted out slowly. On the other hand, ODN cannot penetrate in those pores because it is a larger molecule. Thus, it is eluted quickly through the column.

Size-exclusion Chromatography works well for the separation of ODN from small organic molecules with a minimum volume of eluate. Nevertheless, the method has a problem. The method cannot separate impurities which their sizes are similar to the size of full-length ODN. In addition, the method gives a good resolution when doing small scale. Large scale purification using this chromatography can cause a poor resolution.

\subsubsection{Polyacrylamide Gel Electrophoresis (PAGE)}

PAGE has the best resolution to separate pure ODN from impurities. It is suitable to purify ODN containing more than 60 nucleobases. It can separate and retrieve pure ODN depending on charges on the molecules. The method requires polyacrylamide gel. Despite high purity of ODN, this method is mostly used for qualitative analysis ( $\mu \mathrm{g}-\mathrm{mg}$ ). For purification, only at very small scale is applicable. 


\subsubsection{High Performance Liquid Chromatography (HPLC)}

HPLC is the most popular method for quantitative and qualitative analysis of ODN. The separation using the method is based on the properties of ODN and column absorbents. There are two kinds of HPLC that are used to purify ODN: reverse-phase HPLC and ionexchange HPLC.

\subsubsection{Reverse-phase HPLC (RP-HPLC)}

The separation using RP-HPLC is based on the difference of hydrophobic interaction between absorbent and compound when the sample is eluted by a mobile phase. For absorbent, it is the modified silica containing hydrophobic long-chain alkyl group (eg. $-\mathrm{C}_{18} \mathrm{H}_{37}$ or $\left.-\mathrm{C}_{8} \mathrm{H}_{17}\right)$. There are two standard mobile phases required for elution. One is a

polar mobile phase (usually $0.1 \mathrm{M}$ triethyl ammonium acetate). The other is a non-polar mobile phase (usually $90-100 \%$ acetonitrile). The elution will typically be gradient beginning from $100 \%$ polar mobile phase to $100 \%$ non-polar mobile phase.

After automated synthesis, the 5'-end of full-length ODN contains DMTr group while impurities (failure sequences and small organic molecules) do not. Since DMTr group is hydrophobic, so the interaction of DMTr-on ODN with the absorbent is stronger than that of impurities during gradient elution. Therefore, impurities are eluted out before pure DMTr-on ODN is eluted. After separation, DMTr group on full-length ODN is removed by detritylation (usually $80 \%$ acetic acid) to give pure ODN. However, this method has some drawbacks. It requires large amount of solvent, expensive HPLC column and machine. Also, it is time-consuming to finish purification in the large batches. 


\subsubsection{Ion-exchange HPLC (IX-HPLC)}

IX-HPLC is the other HPLC method that can purify ODN. It is based on the charges interactions between ODN and the ions stationary phase. An additional drawback of this method is the eluent containing non-vaporizable salts. Consequently, the purified ODN needs to be desalted which requires another step before use.

\subsubsection{ODN Cartridge Purification}

The concept of cartridge purification is similar to RP-HPLC. However, pure ODN can be separated from impurities by gravity. So, it does not need high pressure and the HPLC instrument. The drawback of this method is that it is highly expensive when doing high-throughput purification of ODN due to large column, large volume of harmful solvents. Also, the method itself is very slow.

\subsubsection{Affinity Purification}

Affinity purification is the method that separate ODN from impurities by specific interaction. Examples are biotin-avidin affinity purification ${ }^{28}$ and fluorous chromatography. ${ }^{26,29}$

\subsubsection{Biotin-avidin affinity purification}

This method utilizes the interaction between biotin and avidin to purify ODN. During ODN synthesis, full-length ODN is tagged with biotin while the failure sequences are not. Therefore, only full-length ODN will bind with avidin beads in purification process. Failure sequences are washed away and pure full-length ODN is obtained from 
the beads. The problem of this method is that avidin coated beads are very expensive. So, large scale purification could be concerned.

\subsubsection{Fluorous chromatography}

The concept of fluorous chromatography is the same as the Biotin-avidin method. However, the separation is based on the interaction of fluorous-attached ODN and fluorinated absorbent. During automated synthesis, the full-length ODN is tagged with fluorousdimethoxytrityl group but the failure sequences are not. Then, crude ODN is loaded in the Fluoro-Pak column that contains fluorinated organic groups on polymeric resins. Since the full-length ODN (with fluorous tag) has stronger affinity with the absorbent than failure sequences, full-length ODN elutes slower and separate from failure sequences which elute earlier. Nonetheless, the method has disadvantages. It needs large column to scale up the purification which is high cost. In addition, the fluorous column is not reusable. 


\subsection{ODN Characterization}

After purification, ODN are characterized to confirm its identity. ODN is a large and non-volatile molecule. One way to prove the identity of ODN is molecular mass. There are two methods used for obtaining molecular mass of ODN.

\subsubsection{Matrixed Assisted Laser Desorption and Ionization Time-of-Flight (MALDI- TOF) Mass Spectroscopy}

MALDI-TOF is the technique that can be used to analyze mass of biomolecules. ${ }^{30}$ For ODNs, it can determine the molecular mass of sequences up to 50-mer. Sample is usually prepared by mixing the ODN and a matrix homogenously (eg. Dihydroxybenzoic acid (DHB), 3-hydroxypicolinic acid (HPA), $\alpha$-cyano-4-hydroxycinnamic acid (CHCA), Picolinic acid, etc.) in organic solvents. The mixture is put on a plate and the solvents are evaporated to give dried-droplet spot. The target is loaded into MALDI-TOF machine. After that, UV laser irradiates the sample spot. Matrix absorbs UV and transforms it to heat energy. Then, matrix heats quickly and leads ODN desorption. The protonized matrix gives protons to the ODN. Then, ODN becomes ions (Ionization). Since the energy from the laser is not direct to the ODN, the molecular mass can be found in a low fragmentation level. After that, the ions are analyzed by Time of flight (TOF) analyzer which determine molecular mass of ODN by ion flight time. 


\subsubsection{Electrospray Ionization (ESI) Mass Spectroscopy}

ESI is suitable for ODN containing up to 150 nucleobases. ${ }^{31}$ The ODN sample in a form of solution is sprayed and ionized to get charged droplets in the electrospray chamber. Next, the volatile organic solvent on droplets is desolvated to get ions. Afterward, ions with varied charges will travel to detector at different times. Mass to charge ratio of each ion is measured by detector. Then, the result will be shown on a mass spectrum. 


\section{References}

1. Dahm, R., Friedrich Miescher and the discovery of DNA. Developmental Biology 2005, 278 (2), 274-288.

2. Watson, J. D.; Crick, F. H. C., Molecular structure of nucleic acids: A structure for deoxyribose nucleic acid. Nature 1953, 171 (4356), 737-738.

3. Ghosh, A.; Bansal, M., A glossary of DNA structures from A to Z. Acta Crystallographica Section D 2003, 59 (4), 620-626.

4. Yakovchuk, P.; Protozanova, E.; Frank-Kamenetskii, M. D., Base-stacking and base-pairing contributions into thermal stability of the DNA double helix. Nucleic Acids Research 2006, 34 (2), 564-574.

5. (a) Dias, N.; Stein, C. A., Antisense Oligonucleotides: Basic Concepts and Mechanisms. Molecular Cancer Therapeutics 2002, 1 (5), 347-355; (b) RichardtPargmann, D.; Vollmer, J., Stimulation of the Immune System by Therapeutic Antisense Oligodeoxynucleotides and Small Interfering RNAs via Nucleic Acid Receptors. Annals of the New York Academy of Sciences 2009, 1175 (1), 40-54.

6. Mulamba, G. B.; Hu, A.; Azad, R. F.; Anderson, K. P.; Coen, D. M., Human Cytomegalovirus Mutant with Sequence-Dependent Resistance to the Phosphorothioate Oligonucleotide Fomivirsen (ISIS 2922). Antimicrobial Agents and Chemotherapy 1998, $42(4), 971-973$.

7. (a) Crooke, S. T.; Geary, R. S., Clinical pharmacological properties of mipomersen (Kynamro), a second generation antisense inhibitor of apolipoprotein B. British Journal of Clinical Pharmacology 2013, 76 (2), 269-276; (b) Harchaoui, K.; Akdim, F.; Stroes, E. G.; Trip, M.; Kastelein, J. P., Current and Future Pharmacologic Options for the Management 
of Patients Unable to Achieve Low-Density Lipoprotein-Cholesterol Goals with Statins. Am J Cardiovasc Drugs 2008, 8 (4), 233-242; (c) Merki, E.; Graham, M. J.; Mullick, A. E.; Miller, E. R.; Crooke, R. M.; Pitas, R. E.; Witztum, J. L.; Tsimikas, S., Antisense Oligonucleotide Directed to Human Apolipoprotein B-100 Reduces Lipoprotein(a) Levels and Oxidized Phospholipids on Human Apolipoprotein B-100 Particles in Lipoprotein(a) Transgenic Mice. Circulation 2008, 118 (7), 743-753; (d) Athyros, V. G.; Kakafika, A. I.; Tziomalos, K.; Karagiannis, A.; Mikhailidis, D. P., Antisense technology for the prevention or the treatment of cardiovascular disease: The next blockbuster? Expert Opinion on Investigational Drugs 2008, 17 (7), 969-972.

8. (a) Sanger, F.; Coulson, A. R., A rapid method for determining sequences in DNA by primed synthesis with DNA polymerase. Journal of Molecular Biology 1975, 94 (3), 441-448; (b) Sanger, F.; Nicklen, S.; Coulson, A. R., DNA sequencing with chainterminating inhibitors. Proceedings of the National Academy of Sciences 1977, 74 (12), 5463-5467.

9. Bartlett, J. S.; Stirling, D., A Short History of the Polymerase Chain Reaction. In PCR Protocols, Bartlett, J. S.; Stirling, D., Eds. Humana Press: 2003; Vol. 226, pp 3-6.

10. (a) Khorana, H. G.; Agarwal, K. L.; Büchi, H.; Caruthers, M. H.; Gupta, N. K.; Klbppe, K.; Kumar, A.; Ohtsuka, E.; RajBhandary, U. L.; van de Sande, J. H.; Sgaramella, V.; Tebao, T.; Weber, H.; Yamada, T., CIII. Total synthesis of the structural gene for an alanine transfer ribonucleic acid from yeast. Journal of Molecular Biology 1972, 72 (2), 209-217; (b) Itakura, K.; Hirose, T.; Crea, R.; Riggs, A.; Heyneker, H.; Bolivar, F.; Boyer, H., Expression in Escherichia coli of a chemically synthesized gene for the hormone somatostatin. Science 1977, 198 (4321), 1056-1063. 
11. (a) Young, L.; Dong, Q., Two-step total gene synthesis method. Nucleic Acids Research 2004, 32 (7), e59; (b) Chen, G.-Q.; Choi, I.; Ramachandran, B.; Gouaux, J. E., Total Gene Synthesis: Novel Single-Step and Convergent Strategies Applied to the Construction of a 779 Base Pair Bacteriorhodopsin Gene. Journal of the American Chemical Society 1994, 116 (19), 8799-8800.

12. Michelson, A. M.; Todd, A. R., Nucleotides part XXXII. Synthesis of a dithymidine dinucleotide containing a 3 [prime or minute]: 5 [prime or minute]-internucleotidic linkage. Journal of the Chemical Society (Resumed) 1955, 2632-2638.

13. Gilham, P. T.; Khorana, H. G., Studies on Polynucleotides. I. A New and General Method for the Chemical Synthesis of the C5"-C3" Internucleotidic Linkage. Syntheses of Deoxyribo-dinucleotides1. Journal of the American Chemical Society 1958, 80 (23), 62126222.

14. Letsinger, R. L.; Kornet, M. J., Popcorn Polymer as a Support in Multistep Syntheses. Journal of the American Chemical Society 1963, 85 (19), 3045-3046.

15. (a) Letsinger, R. L.; Ogilvie, K. K., Nucleotide chemistry. XIII. Synthesis of oligothymidylates via phosphotriester intermediates. Journal of the American Chemical Society 1969, 91 (12), 3350-3355; (b) Letsinger, R. L.; Ogilvie, K. K.; Miller, P. S., Nucleotide chemistry. XV. Developments in syntheses of oligodeoxyribonucleotides and their organic derivatives. Journal of the American Chemical Society 1969, 91 (12), 33603365.

16. (a) Garegg, P. J.; Lindh, I.; Regberg, T.; Stawinski, J.; Strömberg, R.; Henrichson, C., Nucleoside H-phosphonates. III. Chemical synthesis of oligodeoxyribonucleotides by the hydrogenphosphonate approach. Tetrahedron Letters 1986, 27 (34), 4051-4054; (b) 
Froehler, B. C.; Ng, P. G.; Matteucci, M. D., Synthesis of DNA via deoxynudeoside Hphosphonate Intermediates. Nucleic Acids Research 1986, 14 (13), 5399-5407.

17. Strömberg, R.; Stawinski, J., Synthesis of Oligodeoxyribo- and Oligoribonucleotides According to the H-Phosphonate Method. In Current Protocols in Nucleic Acid Chemistry, John Wiley \& Sons, Inc.: 2001.

18. Letsinger, R. L.; Lunsford, W. B., Synthesis of thymidine oligonucleotides by phosphite triester intermediates. Journal of the American Chemical Society 1976, 98 (12), 3655-3661.

19. Beaucage, S. L.; Caruthers, M. H., Synthetic Strategies and Parameters Involved in the Synthesis of Oligodeoxyribonucleotides According to the Phosphoramidite Method. In Current Protocols in Nucleic Acid Chemistry, John Wiley \& Sons, Inc.: 2001.

20. (a) Matteucci, M. D.; Caruthers, M. H., Synthesis of deoxyoligonucleotides on a polymer support. Journal of the American Chemical Society 1981, 103 (11), 3185-3191; (b) Beaucage, S. L.; Caruthers, M. H., Deoxynucleoside phosphoramidites-A new class of key intermediates for deoxypolynucleotide synthesis. Tetrahedron Letters 1981, 22 (20), 1859-1862; (c) McBride, L. J.; Caruthers, M. H., An investigation of several deoxynucleoside phosphoramidites useful for synthesizing deoxyoligonucleotides. Tetrahedron Letters 1983, 24 (3), 245-248.

21. Benson, F. R., The Chemistry of the Tetrazoles. Chemical Reviews 1947, 41 (1), 161.

22. Vargeese, C.; Carter, J.; Yegge, J.; Krivjansky, S.; Settle, A.; Kropp, E.; Peterson, K.; Pieken, W., Efficient activation of nucleoside phosphoramidites with 4,5- 
dicyanoimidazole during oligonucleotide synthesis. Nucleic Acids Research 1998, 26 (4), 1046-1050.

23. (a) Alul, R. H.; Singman, C. N.; Zhang, G.; Letsinger, R. L., Oxalyl-CPG: a labile support for synthesis of sensitive oligonucleotide derivatives. Nucleic Acids Research 1991, 19 (7), 1527-1532; (b) Hayakawa, Y.; Uchiyama, M.; Noyori, R., Nonaqueous oxidation of nucleoside phosphites to the phosphates. Tetrahedron Letters 1986, 27 (35), 4191-4194.

24. Dellinger, D. J.; Sheehan, D. M.; Christensen, N. K.; Lindberg, J. G.; Caruthers, M. H., Solid-Phase Chemical Synthesis of Phosphonoacetate and Thiophosphonoacetate Oligodeoxynucleotides. Journal of the American Chemical Society 2003, 125 (4), 940-950. 25. Ugi, I.; Jacob, P.; Landgraf, B.; Ruppf, C.; Lemmen, P.; Verfürth, U., Phosphite Oxidation and the Preparation of Five-Membered Cyclic Phosphorylating Reagents Via the Phosphites. Nucleosides and Nucleotides 1988, 7 (5-6), 605-608.

26. Pearson, W. H.; Berry, D. A.; Stoy, P.; Jung, K.-Y.; Sercel, A. D., Fluorous Affinity Purification of Oligonucleotides. The Journal of Organic Chemistry 2005, 70 (18), 71147122.

27. Sawadogo, M.; Dyke, M. W. V., A rapid method for the purification of deprotected oligodeoxynucleotides. Nucleic Acids Research 1991, 19 (3), 674.

28. (a) Fang, S.; Bergstrom, D. E., Fluoride-cleavable biotinylation phosphoramidite for 5'-end-labeling and affinity purification of synthetic oligonucleotides. Nucleic Acids Research 2003, 31 (2), 708-715; (b) Fang, S.; Bergstrom, D. E., Reversible 5'-end biotinylation and affinity purification of synthetic RNA. Tetrahedron Letters 2004, 45 (43), 7987-7990. 
29. Beller, C.; Bannwarth, W., Noncovalent Attachment of Nucleotides by Fluorous

Fluorous In

Synthetic DNA Fragments. Helvetica Chimica Acta 2005, 88 (1), 171-179.

30. Castleberry, C. M.; Chou, C.-W.; Limbach, P. A., Matrix-Assisted Laser Desorption/Ionization Time-of-Flight Mass Spectrometry of Oligonucleotides. In Current Protocols in Nucleic Acid Chemistry, John Wiley \& Sons, Inc.: 2001.

31. Castleberry, C. M.; Rodicio, L. P.; Limbach, P. A., Electrospray Ionization Mass Spectrometry of Oligonucleotides. In Current Protocols in Nucleic Acid Chemistry, John Wiley \& Sons, Inc.: 2001. 


\title{
Chapter 3
}

\section{Scalable Synthetic Oligodeoxynucleotide Purification with Use of a}

\section{Catching by Polymerization, Washing, and Releasing Approach *}

\author{
Shiyue Fang ${ }^{a}$ and Suntara Fueangfung ${ }^{a}$ \\ ${ }^{a}$ Department of Chemistry, Michigan Technological University, 1400 Townsend Drive, \\ Houghton, MI 49931-1295 USA \\ Email: shifang@mtu.edu
}

*The material contained in this chapter was reprinted with permission from Organic Letters 2010 , 12 (16), 3720-3723. Copyright 2010 American Chemical Society. See Appendix D. Permission from publishers. 


\begin{abstract}
Synthetic oligodeoxynucleotides are purified with use of a catching by polymerization, washing, and releasing approach. The method does not require any chromatography, and purification is achieved by simple operations such as shaking, washing, and extraction. It is therefore useful for large-scale purification of synthetic oligonucleotide drugs. In addition to purification of oligonucleotides, this catching by polymerization concept is expected to be equally useful for purification of other synthetic oligomers such as peptides and oligosaccharides.
\end{abstract}




\subsection{Introduction}

There is a high expectation on using synthetic oligonucleotides (ONs) as therapeutics to cure human diseases. ${ }^{1}$ Currently, one such agent is used clinically and more are on various stages of clinical trials. In addition, numerous projects with the goal of developing $\mathrm{ON}$ drugs through selective gene silencing are in progress. As a result, large quantities (kilograms to metric tons) of pure synthetic ONs are needed for preclinical research, clinical trials, and patient $u$ use $^{2}$ Due to the advancement of solid phase synthesis technology, large-scale ON synthesis has become possible. However, the crude ON contains various impurities, which must be removed for most applications. Current purification technologies include trityl-on reverse phase (RP) HPLC, anion exchange HPLC, polyacrylamide gel electrophoresis (PAGE), and various types of affinity chromatography. ${ }^{3}$ These methods are either unsuitable or highly expensive for large-scale purification, and ON purification has been considered as a bottleneck in antisense drug development. $^{2}$ In this communication, we report the use of an unprecedented concept; catching by polymerization; for the development of a simple method for ON purification. ${ }^{4}$ This method is easy to perform and uses inexpensive materials, and therefore is suitable for large-scale purification.

\subsection{Results and Discussion}

The major impurities in crude $\mathrm{ON}$ are the failure sequences generated in the coupling step in each synthetic cycle. For a typical 20-mer oligodeoxynucleotide (ODN) synthesis, the yield of full-length ODN is $40-70 \%$ depending on the scale of the synthesis,

and $30-60 \%$ of ODNs are failure sequences ${ }^{3 \mathrm{f}}$ During automated synthesis, the failure 
sequences are capped with acetic anhydride in each synthetic cycle to prevent them from coupling with phosphoramidite monomers in the next synthetic cycles. We reasoned that if a phosphoramidite that contains a reversibly linked polymerizable function is used in the last synthetic cycle, it will only be coupled to the 5'-end of the full-length ODN. As a result, after synthesis, cleavage, and deprotection, all full-length ODN will contain a polymerizable group while the failure sequences will not. The full-length sequence can then be incorporated into a polymer through a simple copolymerization process, and failure sequences and other impurities can be removed by simple washing. Pure ODN can then be obtained by cleaving from the polymer. If successful, this purification method does not need any chromatography and expensive solid phase affinity extraction materials, and purification can be achieved by simple operations such as shaking, washing, and extraction.

The polymerization reaction we chose for this process was the radical acrylamide polymerization reaction. This reaction uses inexpensive materials, and is widely used by biologists and biochemists for PAGE. The required phosphoramidite 3.1 that contains the polymerizable function (the methacrylamide) and the cleavable linker (the diisopropyl silyl acetal) was synthesized using the route shown in Scheme 3.1. The amino tertiary alcohol 3.3 was prepared by using 3.2 with a known procedure we reported previously. ${ }^{5}$ This compound was acrylated with methacryloyl chloride to give $\mathbf{3 . 4}$, which was coupled with thymidine by diisopropylsilyl bis(trifluoromethane sulfonate) to give 3.5. Phosphinylation of 3.5 with Beaucage's method provided phosphoramidite 3.1. ${ }^{3 \mathrm{a}}$ 


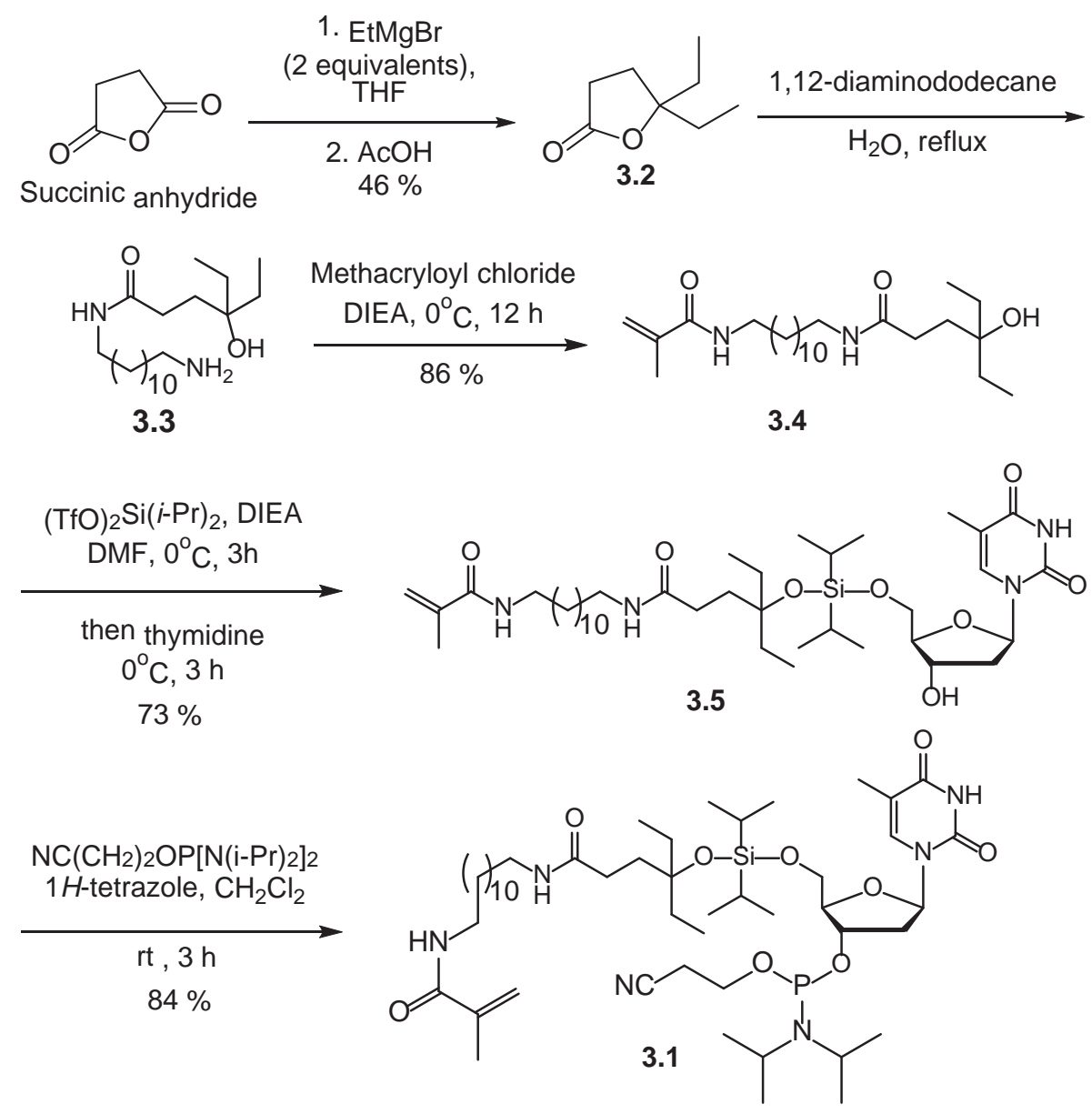

Scheme 3.1. Synthesis of Phosphoramidite 3.1

To prove the purification concept, the 20-mer ODN 3.6 (see Scheme 3.2) was synthesized on a $1 \mu \mathrm{mol}$ scale with standard phosphoramidite chemistry on CPG on a synthesizer. To perform the cleavage and deprotection with ammonium hydroxide at room temperature, the UltraMild DNA synthesis conditions were used (see in the experimental section). ${ }^{6}$ In the last synthetic cycle, phosphoramidite 3.1 was used to incorporate the last nucleotide and the polymerizable methacrylamide group. 
The ODN synthesis result was analyzed with RP HPLC (Figure 3.1). The fulllength sequence 3.6 appeared at $57 \mathrm{~min}$ due to the hydrophobic group at its 5 '-end. Most impurities including failure sequences 3.7 have a retention time between 15 and 20 min.

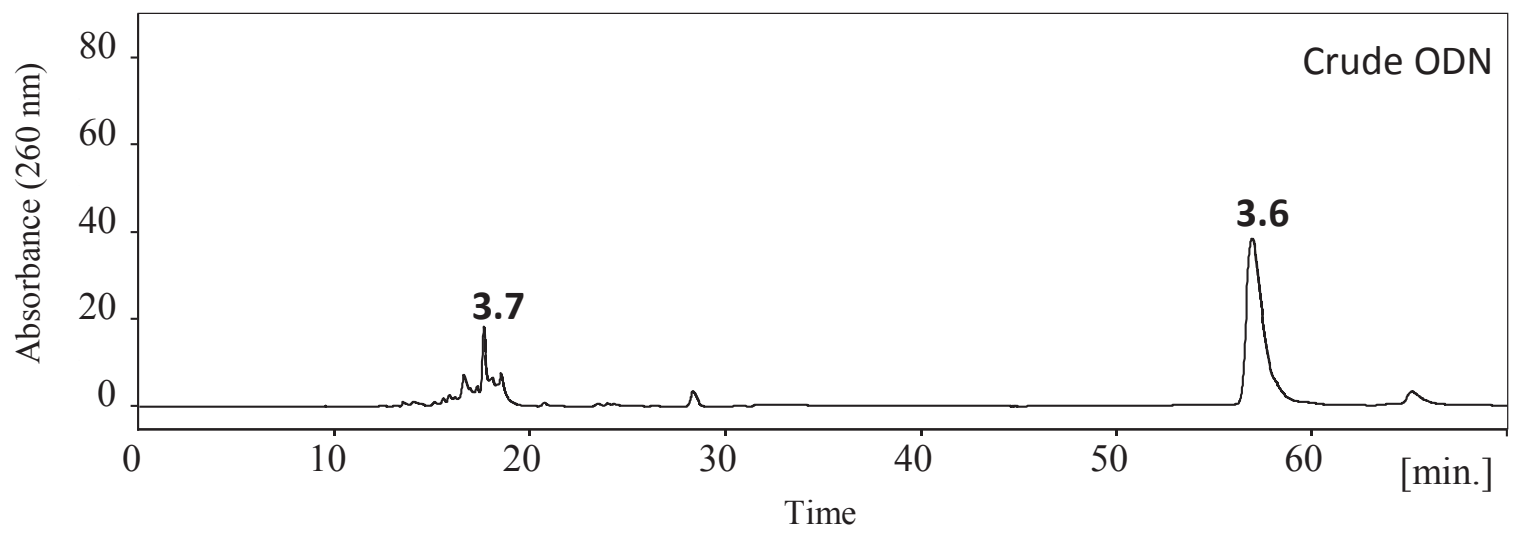

Figure 3.1. RP HPLC profile of crude ODN

For polymerization, the crude ODN ( 50 nmol) was dissolved in water. The polymerization monomer $\mathrm{N}, \mathrm{N}$-dimethylacrylamide and a small amount of cross-linking agent $N, N^{\prime}$-methylenebis(acrylamide) were added. The polymerization was initiated by ammonium persulfate and TMEDA (Scheme 3.2). The reaction is not very sensitive to air but it was performed under a nitrogen atmosphere. The polyacrylamide gel $\mathbf{3 . 8}$ was formed within $30 \mathrm{~min}$ at room temperature. To ensure completion of the reaction, the gel was allowed to stand for another $30 \mathrm{~min}$. At this stage, the full-length ODN $\mathbf{3 . 6}$ was incorporated into the polymer $\mathbf{3 . 8}$ while impurities including failure sequences $\mathbf{3 . 7}$ remained in solution. Water was added to the gel to extract the impurities. The extract was analyzed with RP HPLC. As shown in Figure 3.2, the full-length ODN 3.6 was completely incorporated into the gel. 


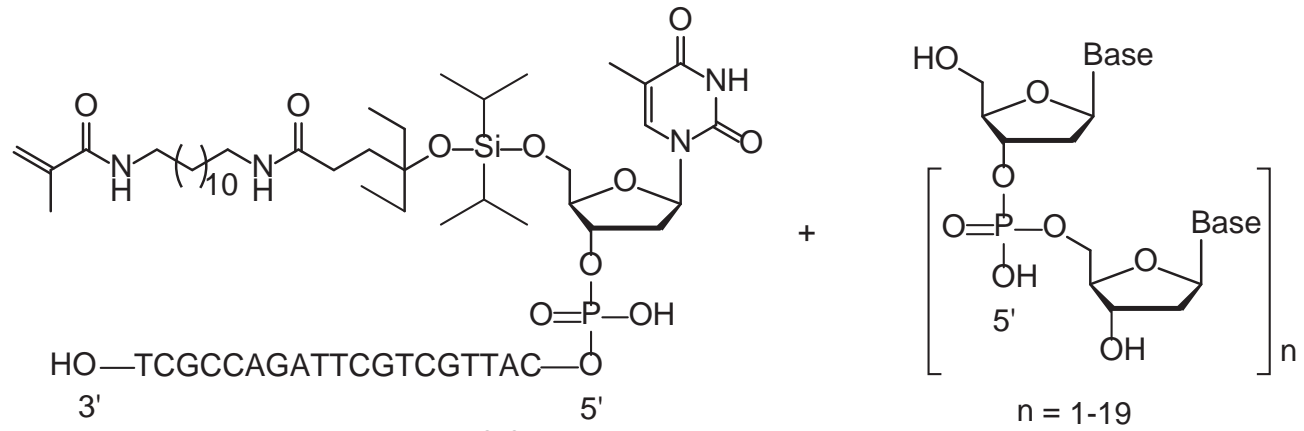

Full-length sequence $\mathbf{3 . 6}$

Failure sequences 3.7 $N, N$ '-methylenebis(acrylamide) $\left(\mathrm{NH}_{4}\right)_{2} \mathrm{~S}_{2} \mathrm{O}_{8}$, TMEDA, $\mathrm{H}_{2} \mathrm{O}$, rt, $1 \mathrm{~h}$

2. Wash with water to remove $\mathbf{3 . 7}$ and other impurities

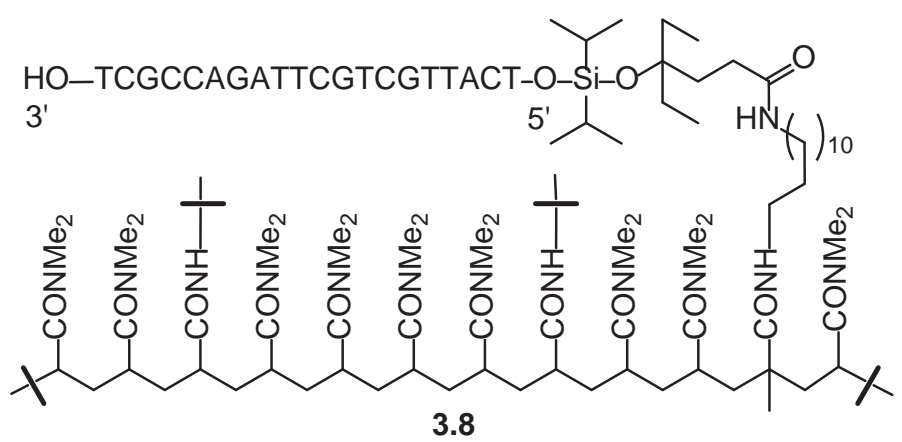

1. HF/pyridine, DMF, rt, 5 h

2. $\mathrm{Me}_{3} \mathrm{SiOMe}, \mathrm{rt}, 30 \mathrm{~min}$

HO_TCGCCAGATTCGTCGTTACT_OH 3.9

$3^{\prime}$

$5^{\prime}$

+ Polymer $+\mathrm{Me}_{3} \mathrm{SiF}+(i-\mathrm{Pr})_{2} \mathrm{SiF}_{2}$

Scheme 3.2. Purification of ODN with use of the catching by polymerization, washing, and releasing approach 


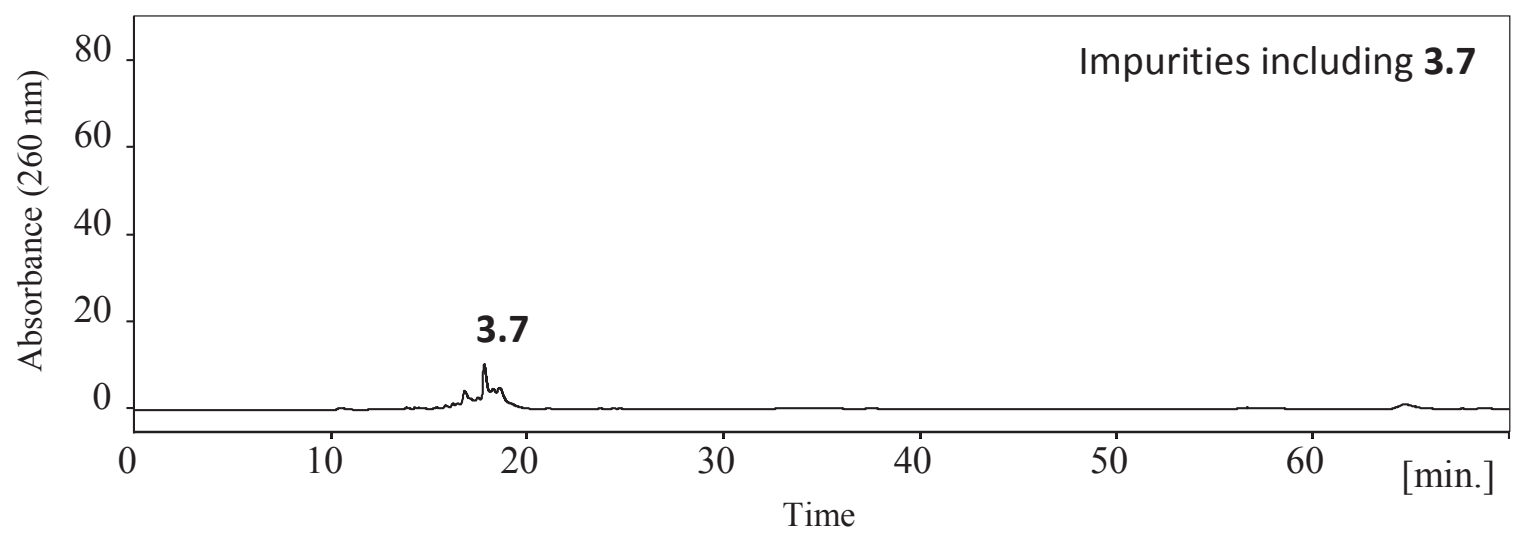

Figure 3.2. RP HPLC profile of impurities including $\mathbf{3 . 7}$

After drying the gel under vacuum thoroughly, the full-length unmodified ODN 3.9 was cleaved with HF-pyridine in DMF at room temperature. Excess HF was quenched with $\mathrm{Me}_{3} \mathrm{SiOMe}$ (Scheme 3.2). The supernatant was removed, and the gel was washed with water. The supernatant and water were combined. Volatiles, which included $\mathrm{Me}_{3} \mathrm{SiF},(i-$ $\operatorname{Pr})_{2} \mathrm{SiF}_{2}, \mathrm{MeOH}$, pyridine, DMF, and water, were evaporated, and the ODN was analyzed with RP HPLC. As shown in trace a (Figure 3.3), failure sequences (3.7) and other impurities were completely removed, and ODN 3.9 was pure. Due to its lower lipophilicity than 3.6, 3.9 has a retention time of $19 \mathrm{~min}$. The recovery yield for the purification process was estimated to be $72 \%$ by comparing the area of the peak in trace a (Figure 3.3) at 19 min with the area of the peak in Figure 3.1 at $57 \mathrm{~min}$. This yield is higher than those typically obtained with trityl-on and fluorous phase purification methods. We anticipated that the yield of this new technology could be further increased when the process is used for larger scale ODN purification. By comparing Figure 3.1, 3.2, and 3.3, we can also conclude that the diisopropyl silyl acetal linker is stable during ODN synthesis, cleave, deprotection, acrylamide gel formation, and extraction of failure sequences. 


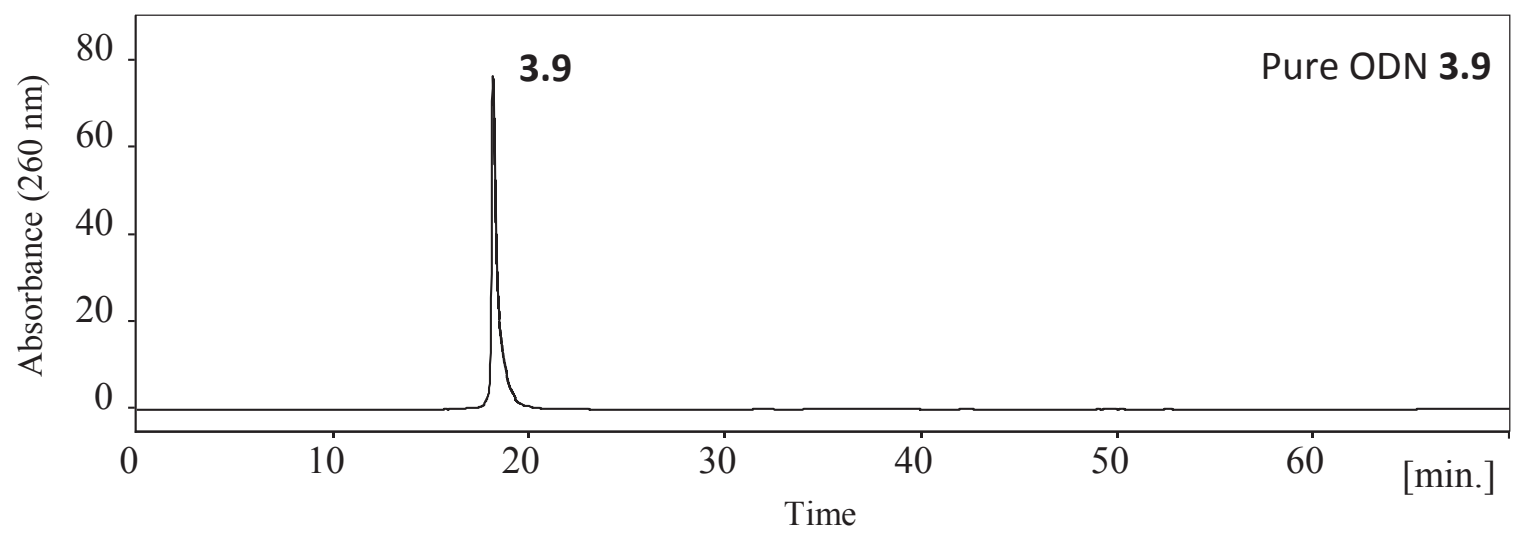

Figure 3.3. RP HPLC profile of ODN 3.9 purified by polymerization, washing, and releasing

The identity of ODN 3.9 was established by comparing with an authentic sample synthesized under standard conditions and purified with RP HPLC by a company (Figure 3.4, trace b). As shown in Figure 3.3 (trace a), a co-injection of $\mathbf{3 . 9}$ purified by using our technology with an authentic sample gave a single peak. The HPLC profile for the authentic sample is shown in trace b of Figure 3.4. MALDI-TOF analysis of $\mathbf{3 . 9}$ also gave correct molecular weight. Because damaged ODNs resulting from nucleobase modifications under radical polymerization conditions may not be able to be detected by HPLC and MALDITOF, the four nucleosides, adenosine, thymidine, guanosine, and cytidine, were subjected to the polymerization conditions, and then recovered from the polyacrylamide gel by extraction. HPLC analysis showed that these compounds are completely stable under the polymerization conditions (see in the experimental section). 

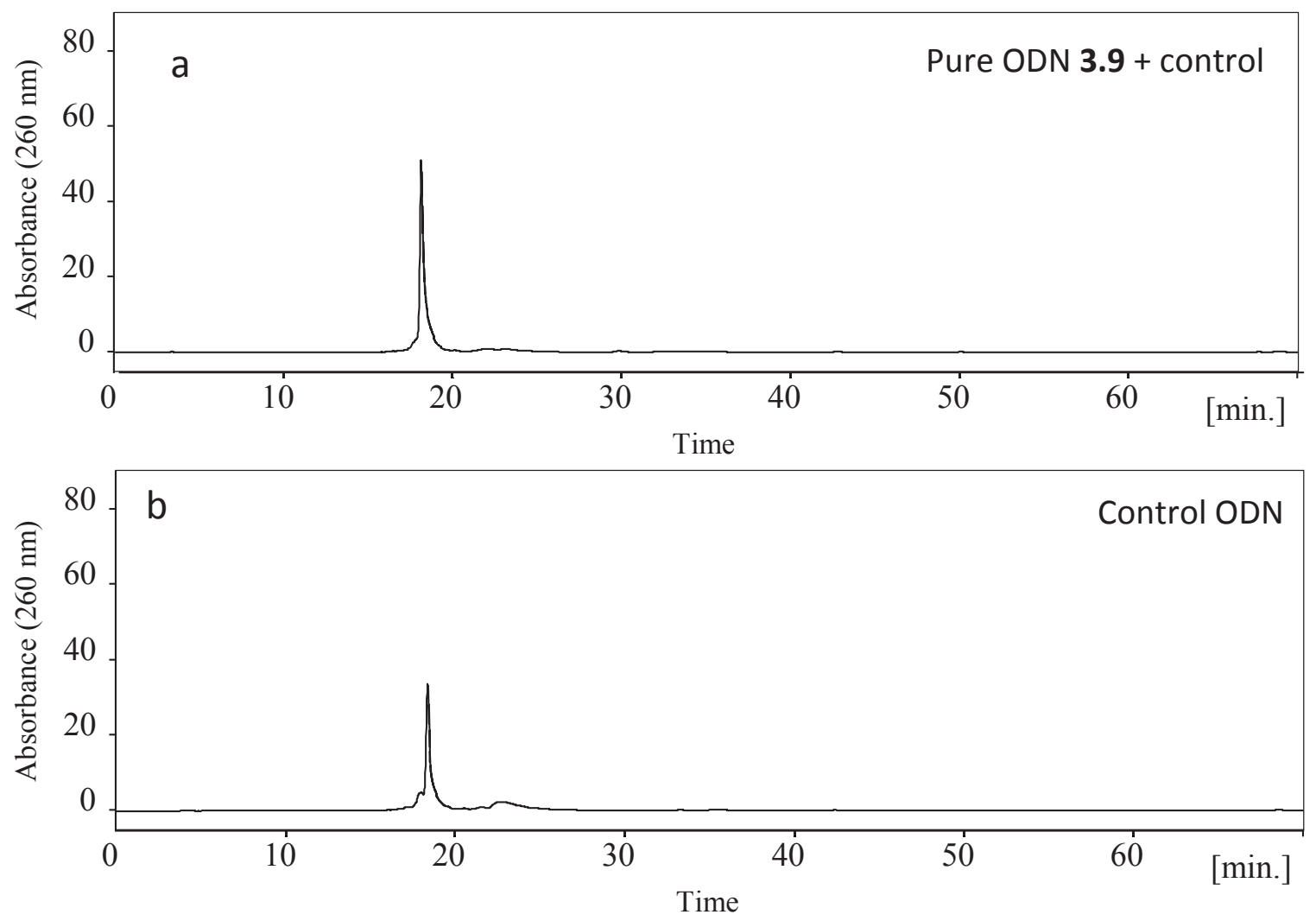

Figure 3.4. RP HPLC profiles: (a) co-injection of ODN 3.9 and control ODN; (b) control ODN

This new ON purification technology has significant advantages over known ones. The most widely used method for ON purification is trityl-on RP HPLC. Compared with this method and other methods that use more hydrophobic tags than $\mathrm{DMTr},{ }^{3 \mathrm{e}}$ our technique does not need any capital expenses including those for the highly expensive and consumable preparative columns. In addition, our technique only needs a minimum amount of organic solvents. The polymerization, removing failure sequences, and extraction of full-length ON are all carried out with water as the solvent. Furthermore, our method is expected to give better results for purification of ONs that are prone to adopt secondary structures and phosphorothioates that contain different diastereoisomers. These materials 
usually gave broad peaks in chromatography. ${ }^{7}$ In the literature, ONs have also been purified with fluorous affinity chromatography ${ }^{3 f, g}$ and biotin-avidin enabled affinity extraction. ${ }^{3 b-d}$ Compared with these methods, our technique does not need any expensive affinity materials such as fluorous affinity column and avidin coated beads. ON purification methods involving capping failure sequences with reagents that contain reactive groups such as a diene were also proposed. ${ }^{8}$ Using these methods, failure sequences could be removed by extraction with dienophile functionalized microspheres through a Diels-Alder reaction. Compared with these methods, our technology does not need any reactive group functionalized microspheres, which could be highly expensive. In addition, due to the intrinsically lower reaction rate of heterogeneous reactions than homogeneous reactions, and the fact that only groups on the surface of solids can participate in reactions and the majority of groups within the solids cannot, to efficiently remove failure sequences, the previously proposed methods require a large excess of solid phase extraction materials, which will prevent them from being used for large-scale ON purification. In contrast, our technology does not rely on any heterogeneous reactions for purification. In principle, the ratio of the polymerization monomer over $\mathrm{ON}$ can be kept to a minimum as long as the steric bulkiness of $\mathrm{ON}$ does not significantly slow down the polymerization. 


\subsection{Conclusion}

We have developed a new method for the purification of synthetic ON. This method is based on an unprecedented concept, which is catching by polymerization. ${ }^{4}$ With use of this method, purification was achieved by simple operations such as shaking, washing, and extraction; and the method does not require any expensive equipment and materials. As a result, it is suitable for large-scale purification. Application of this new concept for the purification of other synthetic oligomers such as RNA, ${ }^{3 \mathrm{~b}}$ modified ON, peptides, ${ }^{9}$ and oligosaccharides ${ }^{10}$ is in progress.

\subsection{Experimental Section}

\section{General Experimental}

All reactions were performed in oven-dried glassware under a nitrogen atmosphere using standard Schlenk techniques. Reagents and solvents available from commercial sources were used as received unless otherwise noted. Toluene, benzene and THF were distilled from $\mathrm{Na}$ /benzophenone ketyl. 1,2-dichloroethane, 1,4-dioxane, $\mathrm{CH}_{2} \mathrm{Cl}_{2}$ and pyridine were distilled over $\mathrm{CaH}_{2}$. Thin layer chromatography (TLC) was performed using Sigma-Aldrich TLC plates, silica gel 60F-254 over glass support, $0.25 \mu \mathrm{m}$ thickness. Flash column chromatography was performed using Selecto Scientific silica gel, particle size 32$63 \mu \mathrm{m}$. Melting points were determined using a MEL-TEMP ${ }^{\circledR}$ melting point apparatus and are uncorrected. GC-MS were measured on GCMS-QP5050A, Shimadzu; column, DB5MS, $0.25 \mu \mathrm{m}$ thickness, $0.25 \mathrm{~mm}$ diameter, $25 \mathrm{~m}$ length; MS, positive EI. ${ }^{1} \mathrm{H},{ }^{13} \mathrm{C}$ and ${ }^{31} \mathrm{P}$ NMR spectra were measured on a Varian UNITY INOVA spectrometer at 400, 100 and $162 \mathrm{MHz}$, respectively; chemical shifts $(\delta)$ were reported in reference to solvent peaks 
(residue $\mathrm{CHCl}_{3}$ at $\delta 7.24 \mathrm{ppm}$ for ${ }^{1} \mathrm{H}$ and $\mathrm{CDCl}_{3}$ at $\delta 77.00 \mathrm{ppm}$ for ${ }^{13} \mathrm{C}$; residue $\mathrm{CHD}_{2} \mathrm{OD}$ at $\delta 3.31$ ppm for ${ }^{1} \mathrm{H}$ and $\mathrm{CD}_{3} \mathrm{OD}$ at $\delta 49.00$ ppm for $\left.{ }^{13} \mathrm{C}\right)$ and $\mathrm{H}_{3} \mathrm{PO}_{4}\left(\right.$ at $\delta 0.00 \mathrm{ppm}$ for $\left.{ }^{31} \mathrm{P}\right)$. High-resolution mass spectra were obtained on a Finnigan Mat 95XL spectrometer. MALDI-TOF mass spectra were obtained on a Shimadzu Biotech Axima CFRplus spectrometer. ODNs were synthesized on an ABI 394 solid phase synthesizer. HPLC was performed on a JASCO LC-2000Plus System, Pump PU-2089Plus Quaternary Gradient Pump, Detector UV-2075Plus. C-18 reverse phase analytical column (100Å, 25034.6 $\mathrm{mm}$ ). Solvent A: $0.1 \mathrm{M}$ triethylammonium acetate, 5\% acetonitrile; solvent B: 90\% acetonitrile; all profiles were generated by detection of absorbance of DNA at $260 \mathrm{~nm}$ using the linear gradient solvent system: solvent B (0-45\%) in solvent A over 60 min at a flow rate of $0.5 \mathrm{~mL} / \mathrm{min}$. Aqueous $\mathrm{NH}_{4} \mathrm{OH}(\sim 29 \%)$, HF-pyridine (HF, 70\%; pyridine, $\sim 30 \%$ ) and $\mathrm{Me}_{3} \mathrm{SiOMe}$ were purchased from Aldrich Inc. THF/pyridine/ $\mathrm{Pac}_{2} \mathrm{O}$, succinic ester linked DMTr-dT-lcaa-CPG (pore size $1000 \AA \quad$ ) and 5'-DMTr, 2cyanoethylphosphoramidites acetyl-dC, Pac-dA, 4-isopropyl-Pac-dG and dT were purchased from Glen Research, Inc.

\section{Synthesis of compound 3.2.}

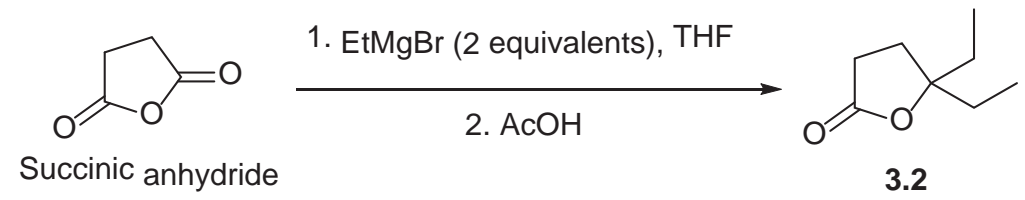

Succinic anhydride (15.0 g, $150 \mathrm{mmol}, 1.0$ eq.) was added in a round bottomed flask, and the flask was flushed with nitrogen. THF $(500 \mathrm{~mL})$ was added via syringe. The solution was cooled to $0{ }^{\circ} \mathrm{C}$. Methyl magnesium bromide in ether (3.0 M, $100 \mathrm{~mL}, 300$ 
mmol, 2.0 eq.) was added via syringe slowly. The reaction mixture was warmed to $\mathrm{rt}$ gradually, and then heated to $50{ }^{\circ} \mathrm{C}$ for $12 \mathrm{~h}$. A yellow solution was formed. After cooling the reaction mixture to $\mathrm{rt}, \mathrm{AcOH}$ was added until $\mathrm{pH}=4$, and stirring was continued for an additional $12 \mathrm{~h}$. Water $(75 \mathrm{~mL})$ was added, and THF and ether were removed under reduced pressure. The green suspension was extracted with $\mathrm{CH}_{2} \mathrm{Cl}_{2}(60 \mathrm{~mL} \times 5)$, and the organic phase was dried over anhydrous $\mathrm{Na}_{2} \mathrm{SO}_{4}$ and concentrated. Purification by vacuum distillation gave $3.2(9.7 \mathrm{~g}, 46 \%)$ as brown oil. This compound has been synthesized using several different methods in the literature. ${ }^{11-13}$

\section{Synthesis of compound 3.3.}

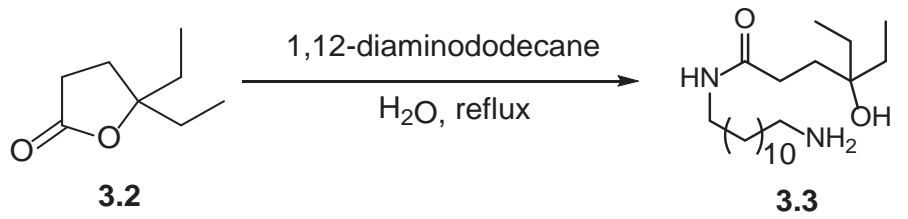

A procedure reported previously by us was followed. ${ }^{3 \mathrm{c}, 5}$ Compound $3.2(2.7 \mathrm{~g}, 19.1$ mmol, 1.0 eq.), 1,12- diaminododecane (9.6 g, $47.7 \mathrm{mmol}, 2.5$ eq.), and water (8.0 mL) were combined. The solution was refluxed under a nitrogen atmosphere overnight. A yellow solution was formed. After cooling to $\mathrm{rt}$, water was removed under reduced pressure. The yellow oily residue was purified by flash column chromatography $\left(\mathrm{SiO}_{2}\right.$, $\left.\mathrm{Et}_{2} \mathrm{O} / \mathrm{CH}_{3} \mathrm{OH} / \mathrm{CH}_{3} \mathrm{CN} / \mathrm{Et}_{3} \mathrm{~N}=5: 2: 2: 1\right)$ to give 3.3 as a light yellow solid $(2.0 \mathrm{~g}, 30 \%)$. $R_{f}=0.5\left(\mathrm{Et}_{2} \mathrm{O} / \mathrm{CH}_{3} \mathrm{OH} / \mathrm{CH}_{3} \mathrm{CN} / \mathrm{Et}_{3} \mathrm{~N}=5: 2: 2: 1\right) ;{ }^{1} \mathrm{H} \mathrm{NMR}\left(400 \mathrm{MHz}, \mathrm{CDCl}_{3}\right) \delta 5.67$ (br s, 1H), 3.23-3.18 (m, 2H), 2.67 (t, 2H, J=7.2 Hz), 2.25 (t, 2H, J=7.2 Hz), 2.02 (br s, $3 \mathrm{H}), 1.74(\mathrm{t}, 2 \mathrm{H}, J=7.2 \mathrm{~Hz}), 1.50-1.40(\mathrm{~m}, 8 \mathrm{H}), 1.33-1.20(\mathrm{~m}, 16 \mathrm{H}), 0.85(\mathrm{t}, 6 \mathrm{H}, J=7.2$ 
$\mathrm{Hz}) ;{ }^{13} \mathrm{C}$ NMR $\left(100 \mathrm{MHz}, \mathrm{CDCl}_{3}\right) \delta 174.2,73.9,42.2,39.9,33.7,31.0,30.9,29.7,29.6$, 29.7, 29.4, 27.1, 27.0, 8.1; HRMS (ESI, [M + H] $]^{+}$) $/ 2$ calculated for $\mathrm{C}_{20} \mathrm{H}_{42} \mathrm{~N}_{2} \mathrm{O}_{2} 343.3325$, found 343.3321 .

\section{Synthesis of compound 3.4.}

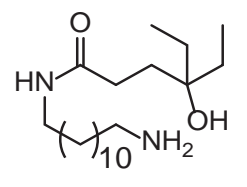

3.3

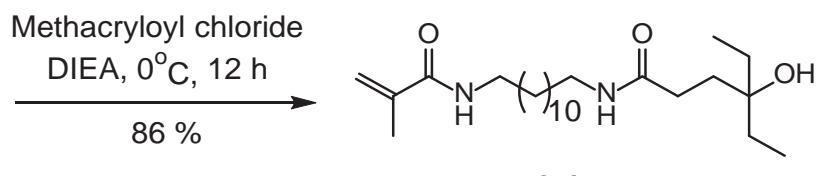

3.4

To a round bottom flask under a nitrogen atmosphere was added diisopropylethylamine ( $750 \mu \mathrm{L}, 4.31 \mathrm{mmol}, 2.5$ eq.) $\mathbf{3 . 3}$ ( $0.6 \mathrm{~g}, 1.75 \mathrm{mmol}, 1.0$ eq.), and $\mathrm{CH}_{2} \mathrm{Cl}_{2}(120 \mathrm{~mL})$. The solution was cooled to $0{ }^{\circ} \mathrm{C}$. Methacrolyl chloride (171 $\mu \mathrm{L}, 1.75$ mmol, 1.0 eq.) was added via a syringe. The flask was then detached from the nitrogen atmosphere and connected to air via a Drierite tube. The reaction mixture was stirred at rt overnight. The content were then transferred into a separation funnel, and were washed with water $(50 \mathrm{~mL})$, which was extracted with $\mathrm{CH}_{2} \mathrm{Cl}_{2}(50 \mathrm{~mL} \times 2)$. The combined organic phase was dried over anhydrous $\mathrm{Na}_{2} \mathrm{SO}_{4}$. Volatiles were then removed under reduced pressure. The crude product was purified by flash column chromatography $\left(\mathrm{SiO}_{2}\right.$, $\mathrm{CH}_{2} \mathrm{Cl}_{2} / \mathrm{CH}_{3} \mathrm{OH}=97$ : 3) giving 3.4 as a white solid $(0.6 \mathrm{~g}, 86 \%) . R_{f}=0.7\left(\mathrm{CH}_{2} \mathrm{Cl}_{2} /\right.$ $\left.\mathrm{CH}_{3} \mathrm{OH}=9: 1\right) ;{ }^{1} \mathrm{H}$ NMR $\left(400 \mathrm{MHz}, \mathrm{CDCl}_{3}\right) \delta 5.85($ br s, $2 \mathrm{H}), 5.63-5.62(\mathrm{~m}, 1 \mathrm{H}), 5.28-$ $5.27(\mathrm{~m}, 1 \mathrm{H}), 3.26(\mathrm{dt}, 2 \mathrm{H}, J=8.4,6.0 \mathrm{~Hz}), 3.18(\mathrm{dt}, 2 \mathrm{H}, J=6.8,6.0 \mathrm{~Hz}), 2.52(\mathrm{br} \mathrm{s}, 1 \mathrm{H})$, $2.25(\mathrm{t}, 2 \mathrm{H}, J=7.2 \mathrm{~Hz}), 1.93(\mathrm{dd}, 3 \mathrm{H}, J=1.2,0.8 \mathrm{~Hz}), 1.73(\mathrm{t}, 2 \mathrm{H}, J=7.2 \mathrm{~Hz}), 1.52-1.41$ (m, 8H), 1.31-1.20 (m, 16H), $0.83(\mathrm{t}, 6 \mathrm{H}, J=7.6 \mathrm{~Hz}) ;{ }^{13} \mathrm{C} \mathrm{NMR}\left(100 \mathrm{MHz}, \mathrm{CDCl}_{3}\right) \delta$ 
174.2, 168.6, 140.5, 119.3, 73.9, 39.9, 33.6, 31.1, 30.9, 29.7, 29.6, 29.4, 27.1, 18.9, 8.1;

HRMS (ESI, $[\mathrm{M}+\mathrm{H}]^{+}$) $\mathrm{m} / z$ calculated for $\mathrm{C}_{24} \mathrm{H}_{46} \mathrm{~N}_{2} \mathrm{O}_{3} 411.3587$, found 411.3582 .

\section{Synthesis of compound 3.5.}

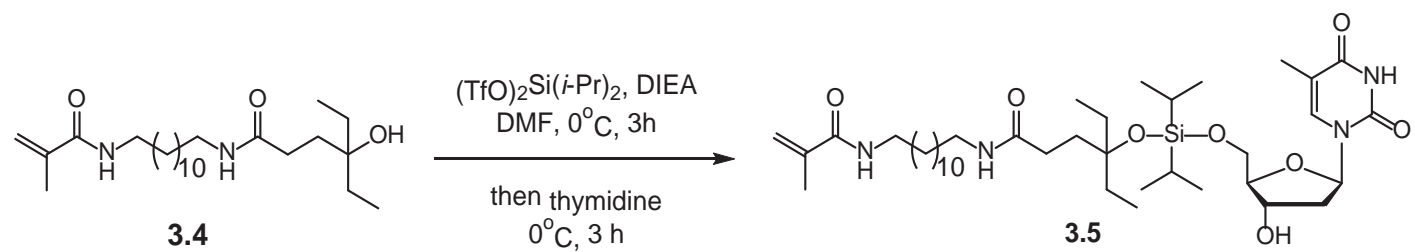

Compound 3.4 (274 mg, $1.0 \mathrm{mmol}, 1.0 \mathrm{eq}$ ) in a 2-necked round bottomed flask was dried under vacuum. The flask was then refilled with nitrogen. The vacuum-nitrogen cycle was repeated for two additional times. Dry DMF ( $5 \mathrm{~mL})$ and diisopropylethylamine (350 $\mu \mathrm{L}, 3.0 \mathrm{mmol}, 3.0 \mathrm{eq}$ ) were added via a syringes. After cooling to $0^{\circ} \mathrm{C}$, diisopropyl silyl bis(trifluoromethane sulfonate) $(196 \mu \mathrm{L}, 1.0 \mathrm{mmol}, 1.0 \mathrm{eq})$ was added via a syringe in one portion. The solution was stirred at $0^{\circ} \mathrm{C}$ for $1 \mathrm{~h}$ and $\mathrm{rt}$ for $2 \mathrm{~h}$. The reaction mixture was then cooled to $0^{\circ} \mathrm{C}$ again. A solution of thymidine $(162 \mathrm{mg}, 1.0 \mathrm{mmol}, 1.0 \mathrm{eq})$ in dry DMF $(1.5 \mathrm{~mL})$ were added via a cannula. After stirring at $0^{\circ} \mathrm{C}$ for $3 \mathrm{~h}$, DMF was removed under vacuum. To the residue, ice-cooled EtOAc $(60 \mathrm{~mL})$ and $\mathrm{NaHCO}_{3}$ solution $(5 \%, 50 \mathrm{~mL})$ were added, and the phases separated. The aqueous phase was further extracted with icecooled EtOAc $\left(0^{\circ} \mathrm{C}, 50 \mathrm{~mL} \times 4\right)$. The organic phase was dried over anhydrous $\mathrm{Na}_{2} \mathrm{SO}_{4}$. EtOAc was removed under reduced pressure to give the crude product as a yellow oil, which was purified by flash column chromatography $\left(\mathrm{SiO}_{2}, \mathrm{CH}_{2} \mathrm{Cl}_{2} / \mathrm{CH}_{3} \mathrm{OH}\right.$ from $100: 0$ to $95: 5)$ giving 3.5 as a white foam (370 mg, $73 \%)$. $R_{f}=0.6\left(\mathrm{CH}_{2} \mathrm{Cl}_{2} / \mathrm{CH}_{3} \mathrm{OH}=9: 1\right.$,); ${ }^{1} \mathrm{H}$ NMR (400 MHz, CD 30 OD) $\delta 7.92$ (br s, $\left.1 \mathrm{H}\right), 7.82$ (br t, $1 \mathrm{H}, J=5.2 \mathrm{~Hz}$ ), 7.49 (s, 1H), 
$6.24(\mathrm{dd}, 1 \mathrm{H}, J=7.2,3.2 \mathrm{~Hz}), 5.64(\mathrm{~s}, 1 \mathrm{H}), 5.32(\mathrm{~s}, 1 \mathrm{H}), 4.42(\mathrm{dt}, 1 \mathrm{H}, J=6.4,2.8 \mathrm{~Hz})$ 4.02-3.91 (m, 3H), 3.24-3.16 (m, 2H), 3.16-3.09 (m, 2H), 2.29-2.13 (m, 4H), 1.91 (dd, 3H, $J=1.6,0.8 \mathrm{~Hz}), 1.86(\mathrm{~d}, 3 \mathrm{H}, J=1.2 \mathrm{~Hz}), 1.86-1.81(\mathrm{~m}, 2 \mathrm{H}), 1.66-1.40(\mathrm{~m}, 8 \mathrm{H}), 1.36-1.24$ (m, 16H), 1.12-1.00 (m, 14H), 0.90 (t, 6H, $J=7.2 \mathrm{~Hz}) ;{ }^{13} \mathrm{C} \mathrm{NMR}\left(100 \mathrm{MHz}, \mathrm{CD}_{3} \mathrm{OD}\right) \delta$ 174.6, 170.0, 165.0, 151.0, 140.3, 136.2, 118.9, 110.3, 87.3, 84.8, 79.0, 70.7, 62.9, 39.8, $39.5,39.2,34.7,31.61,31.57,30.7,29.5,29.3,26.8,17.7,17.42,17.36,17.32,17.29,13.8$, 13.7, 11.5, 7.7; HRMS (ESI, $[\mathrm{M}+\mathrm{H}]^{+}$) calculated for $\mathrm{C}_{40} \mathrm{H}_{72} \mathrm{~N}_{4} \mathrm{O}_{8} \mathrm{Si}$ 765.5198, found 765.5186.

\section{Synthesis of compound 3.1.}

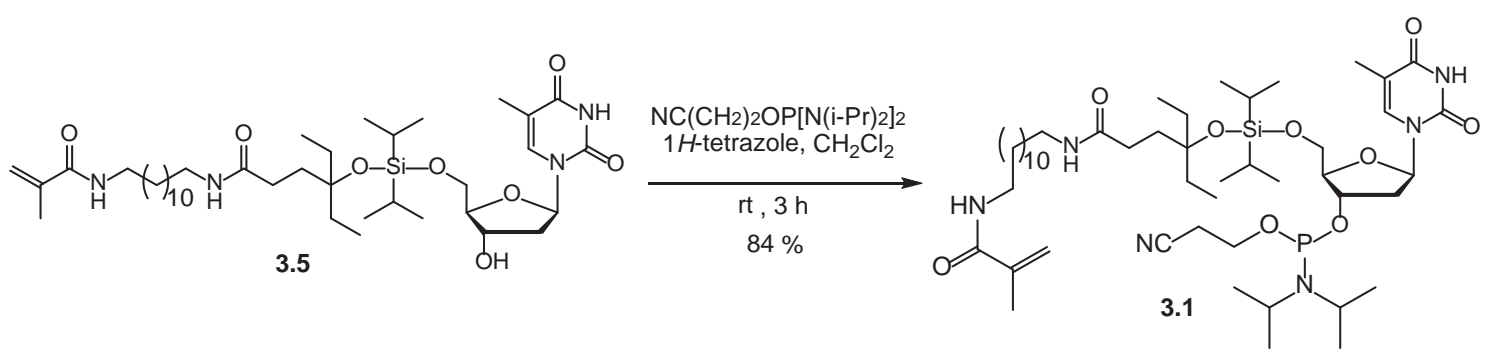

A round-bottomed flask containing $\mathbf{3 . 5}(150 \mathrm{mg}, 0.20 \mathrm{mmol}, 1.0 \mathrm{eq}$.) was flushed with nitrogen. Dry $\mathrm{CH}_{2} \mathrm{Cl}_{2}(6 \mathrm{~mL})$ and 2-cyanoethyl- $N, N, N^{\prime}, N^{\prime}$ - tetraisopropyl phosphoramidite ( $68 \mu \mathrm{L}, 0.22 \mathrm{mmol}, 1.1$ eq.) were added via a syringe sequentially. To the resulting solution was added the solution of $1 \mathrm{H}$-tetrazole in $\mathrm{CH}_{3} \mathrm{CN}(0.45 \mathrm{M}, 480 \mu \mathrm{L}, 0.22$ mmol, 1.1 eq.) in three portions over a period of $1 \mathrm{~h}$. After stirring at $\mathrm{rt}$ for another $2 \mathrm{~h}$, the reaction mixture was concentrated under reduced pressure. The residue was purified by flash chromatography (hexanes $/ \mathrm{CH}_{2} \mathrm{Cl}_{2} / \mathrm{Et}_{3} \mathrm{~N}=1: 2: 0.3$ ), giving 3.1 as a white foam (158 $\mathrm{mg}, 84 \%) . R_{f}=0.5$ (hexanes/ $\left.\mathrm{CH}_{2} \mathrm{Cl}_{2} / \mathrm{Et}_{3} \mathrm{~N}=1: 2: 0.3\right) ;{ }^{1} \mathrm{H} \mathrm{NMR}\left(400 \mathrm{MHz}, \mathrm{CDCl}_{3}\right) \delta$ 
$7.37(\mathrm{~s}, 0.7 \mathrm{H}), 7.32(\mathrm{~s}, 0.3 \mathrm{H}), 6.29(\mathrm{dd}, 1 \mathrm{H}, J=8.0,6.4 \mathrm{~Hz}), 5.63(\mathrm{~s}, 1 \mathrm{H}), 5.27(\mathrm{q}, 1 \mathrm{H}, J=$ $1.2 \mathrm{~Hz}), 4.61-4.52(\mathrm{~m}, 1 \mathrm{H}), 4.18-3.52(\mathrm{~m}, 7 \mathrm{H}), 3.32-3.21(\mathrm{~m}, 2 \mathrm{H}), 3.21-3.10(\mathrm{~m}, 2 \mathrm{H}), 2.67-$ $2.56(\mathrm{~m}, 2 \mathrm{H}), 2.50-2.34(\mathrm{~m}, 1 \mathrm{H}), 2.24-2.14(\mathrm{~m}, 2 \mathrm{H}), 2.13-2.02(\mathrm{~m}, 1 \mathrm{H}), 1.95-1.91(\mathrm{~m}, 3 \mathrm{H})$ $1.87(\mathrm{~s}, 3 \mathrm{H}), 1.87-1.79(\mathrm{~m}, 2 \mathrm{H}), 1.64-1.38(\mathrm{~m}, 8 \mathrm{H}), 1.30-1.10(\mathrm{~m}, 28 \mathrm{H}), 1.10-0.95(\mathrm{~m}, 14 \mathrm{H})$, $0.85(\mathrm{t}, 6 \mathrm{H}, J=7.2 \mathrm{~Hz}) ;{ }^{13} \mathrm{C} \mathrm{NMR}\left(100 \mathrm{MHz}, \mathrm{CDCl}_{3}\right) \delta 173.1,168.6,163.8,150.4,140.5$, $135.5,119.2,117.8,111.2,86.7,86.3(\mathrm{~d}, J=6.1 \mathrm{~Hz}), 84.8,84.6,79.3,73.6(\mathrm{~d}, J=18.3$ Hz), 62.8, $58.3(\mathrm{~d}, J=18.0 \mathrm{~Hz}), 43.5(\mathrm{~d}, J=12.2 \mathrm{~Hz}), 39.9,39.7,34.7,34.6,32.0,31.9$, $31.2,29.8,29.75,29.67,29.46,29.43,27.1,24.84,24.77,24.74,24.6,20.6,18.9,18.29$, $18.26,18.13,18.06,14.1,14.0,13.7,12.5,8.7 ;{ }^{31} \mathrm{P} \mathrm{NMR}\left(160 \mathrm{MHz}, \mathrm{CDCl}_{3}\right) \delta 150.1,149.8$

\section{Synthesis of ODN 3.6}

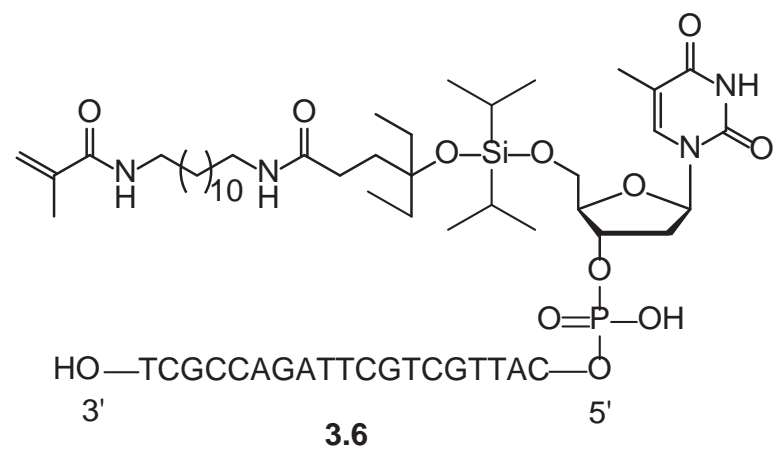

ODN 3.6 was synthesized on an ABI 394 solid phase synthesizer using standard phosphoramidite chemistry under UltraMild conditions on controlled pore glass $(\mathrm{CPG}$, pore size $1000 \AA$ ) on a $1 \mu \mathrm{mol}$ scale. The succinyl ester linkage was used to anchor the ON to CPG. The phosphoramidite monomers used were Pac-dA-CE, Ac-dC-CE, $i$-Pr-Pac-dGCE and dT-CE. Manufacture recommended synthetic cycles were adopted. In the last synthetic cycle, phosphoramidite 3.1 (in acetonitrile, $0.1 \mathrm{M}$, which is two times of normal 
phosphoramidite concentration) was coupled to $5^{\prime}$-end of the $\mathrm{ON}$ for $5 \mathrm{~min}$. Detritylation was not performed in the last synthetic cycle. Cleavage and deprotection were carried out on the synthesizer with concentrated $\mathrm{NH}_{4} \mathrm{OH}(900 \mathrm{~min} \times 4)$ at $\mathrm{rt}$. The solution was distributed equally into 20 Eppendorf tubes $(1.5 \mathrm{~mL})$, and dried in a SpeedVac, separately (each portion contained $\left.\sim 50 \mathrm{nmol} \mathrm{ON}, P_{1}-P_{20}\right)$. $P_{1}$ was dissolved in $80 \mu \mathrm{L}$ water, $20 \mu \mathrm{L}$ $(\sim 12.5 \mathrm{nmol})$ was injected into RP HPLC to generate the profile (Figure 3.1).

\section{Catching by polymerization-synthesis of ODN-polyacrylamide conjugate 3.8}

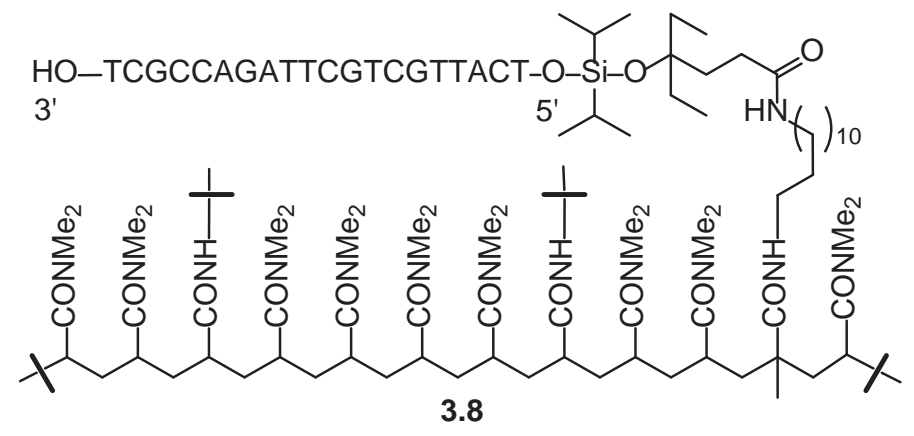

The crude ON 3.6 $\left(P_{2}\right)$ was transferred into a $25 \mathrm{~mL}$ 2-necked round bottomed flask by dissolving into water $(100 \mu \mathrm{L}, 50 \mu \mathrm{L} \times 3)$. To the flask was also added aqueous dimethylacrylamide solution $\quad\left(250 \mu \mathrm{L} ; \quad N, N\right.$-dimethylacrylamide $1.69 \mathrm{M}, \quad N, N^{\prime}$ methylenebis(acrylamide) $16.9 \mathrm{mM}$; the solution could be stored at $-20{ }^{\circ} \mathrm{C}$ in dark for 1 month). The flask was flushed with nitrogen for 2 min with gentle stirring. $\left(\mathrm{NH}_{4}\right) \mathrm{S}_{2} \mathrm{O}_{8}$ $(10 \%, 5 \mu \mathrm{L})$ and $N, N, N^{\prime}, N^{\prime}$-tetramethylethylenediamine (TMEDA, $\left.5 \mu \mathrm{L}\right)$ were added sequentially via pipettes under positive nitrogen pressure. The solution was stirred gently under nitrogen at rt. A gel, which was the ON-polyacrylamide conjugate 3.8, was formed 
within $30 \mathrm{~min}$, which was allowed to stand for another $30 \mathrm{~min}$ to ensure completion of polymerization (Scheme 3.2).

\section{Washing-removal of failure sequences 3.7 and other impurities}

The gel $\mathbf{3 . 8}$ in the 2-necked round bottomed flask was broken into smaller pieces with a glass rod. Water $(5 \mathrm{~mL})$ was added via a pipette. The content was gently stirred overnight (gentle shaking may be better) at rt. The supernatant, which contains failure sequences 3.7 and other impurities, was removed using a pipette (on large scale, filtration should be more convenient). To analyze the efficiency of the catching by polymerization

process, the supernatant was concentrated to $\sim 1 \mathrm{~mL}$ and desalted using a D-Salt ${ }^{\mathrm{TM}}$ dextran desalting column (5K MWCO). After concentration to dryness in a SpeedVac concentrator, $80 \mu \mathrm{L}$ water was added, $20 \mu \mathrm{L}$ was injected into RP HPLC to generate the profile (Figure 3.2). The gel was further rinsed with water $(3 \mathrm{~mL} / 1 \mathrm{~h} \times 3)$ to ensure complete removal of impurities.

\section{Releasing-cleavage of full-length ODN 3.9 from polymer}

\section{3' HO-TCGCCAGATTCGTCGTTAC-OH 5' (3.9)}

The gel was transferred into a $1.5 \mathrm{~mL}$ Eppendorf tube and dried under vacuum overnight. To the tube was added dry DMF $(1 \mathrm{~mL})$ and HF-pyridine $(30 \mu \mathrm{L})$, the tube was vortexed shortly, and then was allowed to stand for $5 \mathrm{~h}$ at rt. At this stage, ODN 3.9 was cleaved from polymer (Scheme 3.2). The supernatant was transferred into another 
Eppendorf tube. To the gel was added $\mathrm{Me}_{3} \mathrm{SiOMe}(300 \mu \mathrm{L})$. After standing at $\mathrm{rt}$ for $30 \mathrm{~min}$, $\mathrm{Me}_{3} \mathrm{SiOMe}$ was combined with the supernatant, and mixture was stand at $\mathrm{rt}$ for a minimum of $15 \mathrm{~min}$. The gel was transferred into a round bottomed flask and water $(5 \mathrm{~mL})$ was added. The mixture was stirred gently at $\mathrm{rt}$ for $12 \mathrm{~h}$. The supernatant was removed via a pipette. The gel was further extracted with water $(3 \mathrm{~mL} / 2 \mathrm{~h} \times 2)$. All supernatants (DMF, $\mathrm{Me}_{3} \mathrm{SiOMe}$ and water) were combined and dried to dryness. The residue was dissolved in $80 \mu \mathrm{L}$ water, $20 \mu \mathrm{L}$ was injected into RP HPLC to generate the profile in Figure 3.3. The recover yield for the purification process was estimated to be $72 \%$ by comparing the area of the peak in Figure 3.3 at 19 min with the area of the peak in Figure 3.1 at 57 min.

\section{Identification of ODN 3.9}

The authentic ODN of $\mathbf{3 . 9}$ was synthesized using standard phosphoramidite chemistry on a $1 \mu \mathrm{mol}$ scale and purified with trityl-on RP HPLC at The Midland Certified Reagent Company, Inc. (Midland, TX, USA). MALDI-TOF spectrum of the authentic ODN was also obtained at the company showing correct molecular weight: calculated for $[\mathrm{M}-\mathrm{H}]^{-} 6057$, found 6060. At Michigan Tech, the authentic sample was divided equally into 20 portions (each portion contains $\sim 50 \mathrm{nmol} \mathrm{ODN}$ assuming the yield for the synthesis and purification was $100 \%$ ). One portion was dissolved in $80 \mu \mathrm{L}$ water, $20 \mu \mathrm{L}$ was injected into RP HPLC to generate trace b (Figure 3.4). To compare ODN 3.9 synthesized in our lab and purified using our catching by polymerization, washing and releasing technique with the authentic sample, $10 \mu \mathrm{L}$ of the solution used to generate trace in Figure 3.3 and $10 \mu \mathrm{L}$ of the solution 
used to generate trace b in Figure 3.4 were mixed, and injected into RP HPLC to generate trace a (Figure 3.4). A single peak was observed showing the two were identical. ODN 3.9 was also characterized with MALDI-TOF: calculated for $[\mathrm{M}-\mathrm{H}]^{-} 6057$, found: 6057 . In addition, two other peaks were also observed in the mass spectrum: calculated for $[\mathrm{M}-2 \mathrm{H}$ - adenine $]^{-}$5922, found: 5925; and calculated for $[\mathrm{M}-2 \mathrm{H}]^{2-} 3028$, found: $3030 .{ }^{14}$ The spectrum is attached in the MALDI-TOF mass spectrum of ODN 3.9 purified using the catching by polymerization, washing and releasing approach section of appendix A.

\section{Testing the stability of nucleobases under radical acrylamide polymerization}

\section{conditions}

$5 \mathrm{mg}$ of guanosine $(\mathrm{dG})$ was charged into a 2-necked round bottomed flask. Water $(250 \mu \mathrm{L})$ was added. The material could not dissolve, so DMF $(250 \mu \mathrm{L})$ was added. After gentle heating with a heat gun, a solution was formed. After cooling to rt,

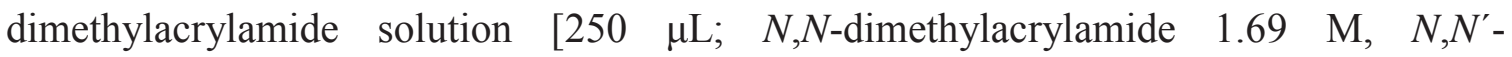
methylenebis(acrylamide) $16.9 \mathrm{mM}$ ] was added. The flask was flushed with nitrogen for $\sim 3$ min. $\left(\mathrm{NH}_{4}\right)_{2} \mathrm{~S}_{2} \mathrm{O}_{8}$ solution $(10 \%, 5 \mu \mathrm{L})$ and TMEDA $(5 \mu \mathrm{L})$ were added, and the mixture was gently stirred at $\mathrm{rt}$. The polymerization was allowed to proceed for $1 \mathrm{~h}$. Water $(5 \mathrm{~mL})$ was added to extract dG (rt, $3 \mathrm{~h}$, gentle stirring). The extract was removed. The gel was further extracted with DMF (rt, 6 h, gentle stirring, $2 \mathrm{~mL} \times 2$ ). All extracts were combined and evaporated to dryness in a SpeedVac concentrator. The residue was dissolved in 15 mL buffer A, $20 \mu \mathrm{L}$ were injected into HPLC (eluting conditions described in the general experimental section were used) to generate HPLC profile Recovered $d G$ (Figure 3.5). 


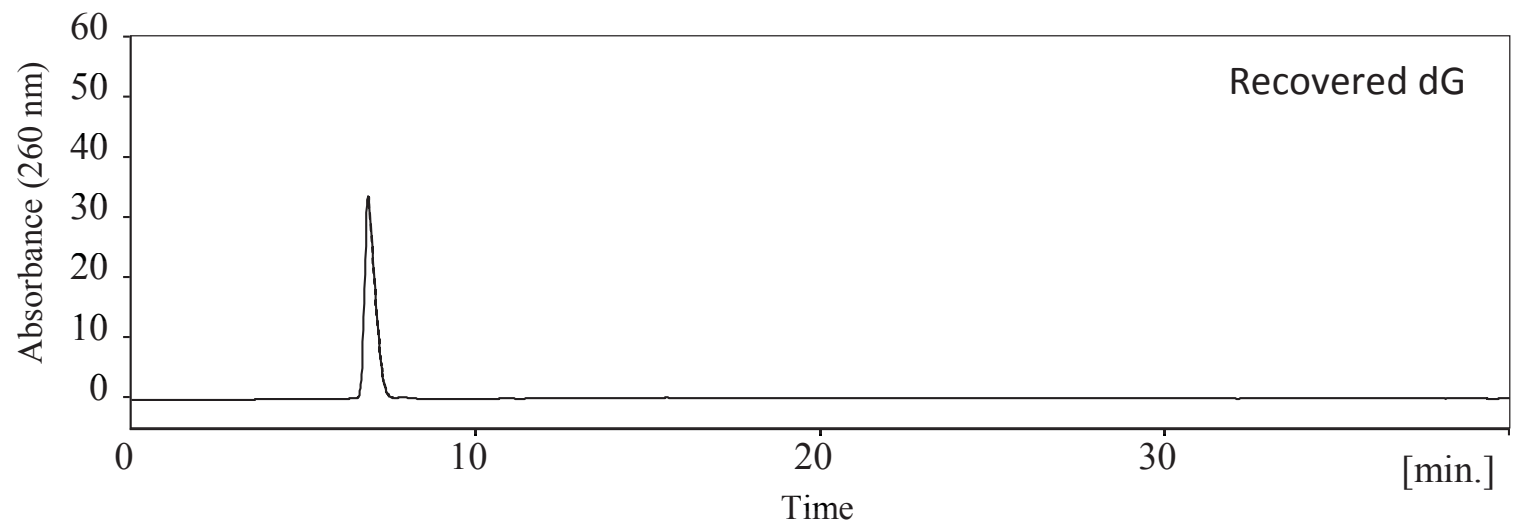

Figure 3.5. HPLC profile of recovered guanosine

The authentic dG $(2.5 \mathrm{mg})$ was dissolved in $5 \mathrm{~mL}$ buffer A, $20 \mu \mathrm{L}$ were injected into HPLC to generate HPLC profile Authentic $d G$ (Figure 3.6).

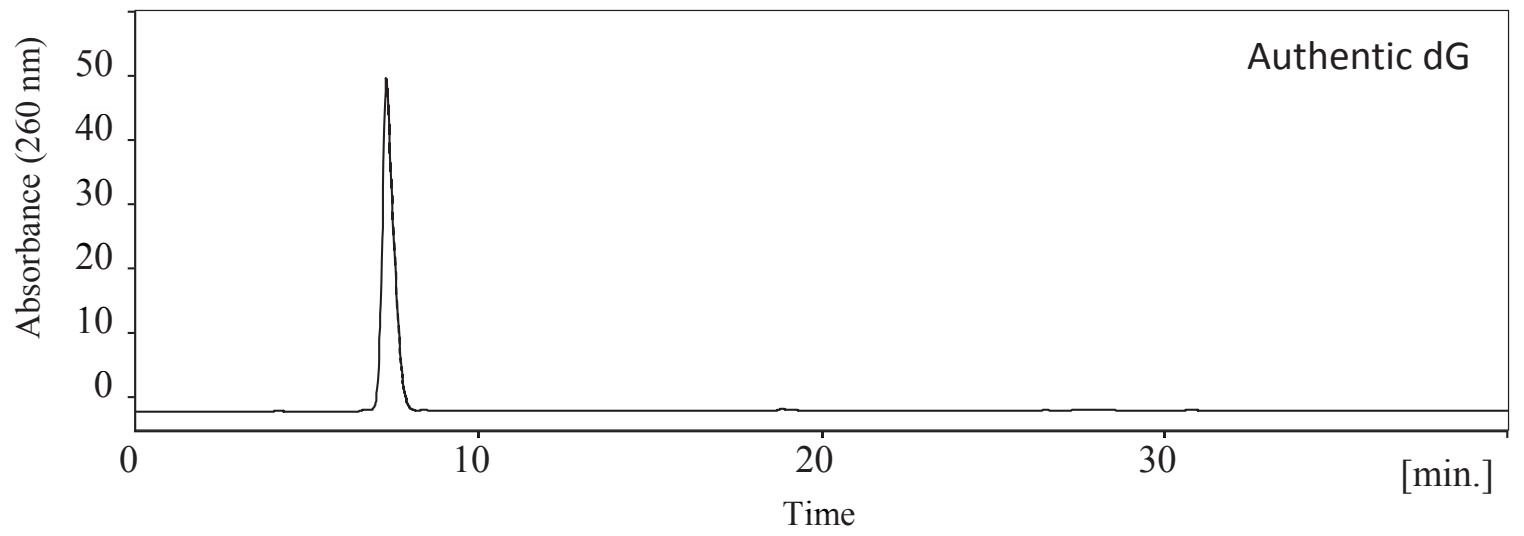

Figure 3.6. HPLC profile of authentic guanosine

Commercially available 8-oxo-guanosine $(1 \mathrm{mg})$ was dissolved in $8 \mathrm{~mL}$ buffer A, $20 \mu \mathrm{L}$ were injected into HPLC to generate HPLC profile Authentic 8-oxo-dG (Figure 3.7). 


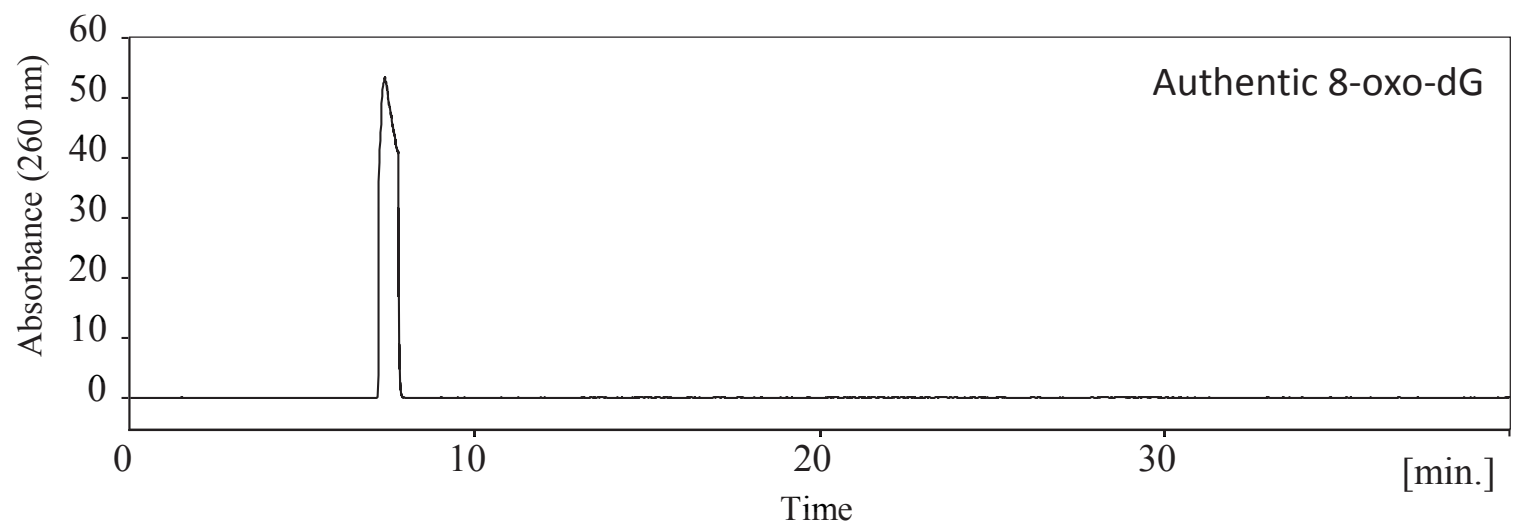

Figure 3.7. HPLC profile of authentic 8-oxo-guanosine

The co-injection HPLC profile of recovered guanosine and authentic guanosine was generated using $10 \mu \mathrm{L}$ of each solution. A single peak was observed, which indicated that they are identical (Figure 3.8).

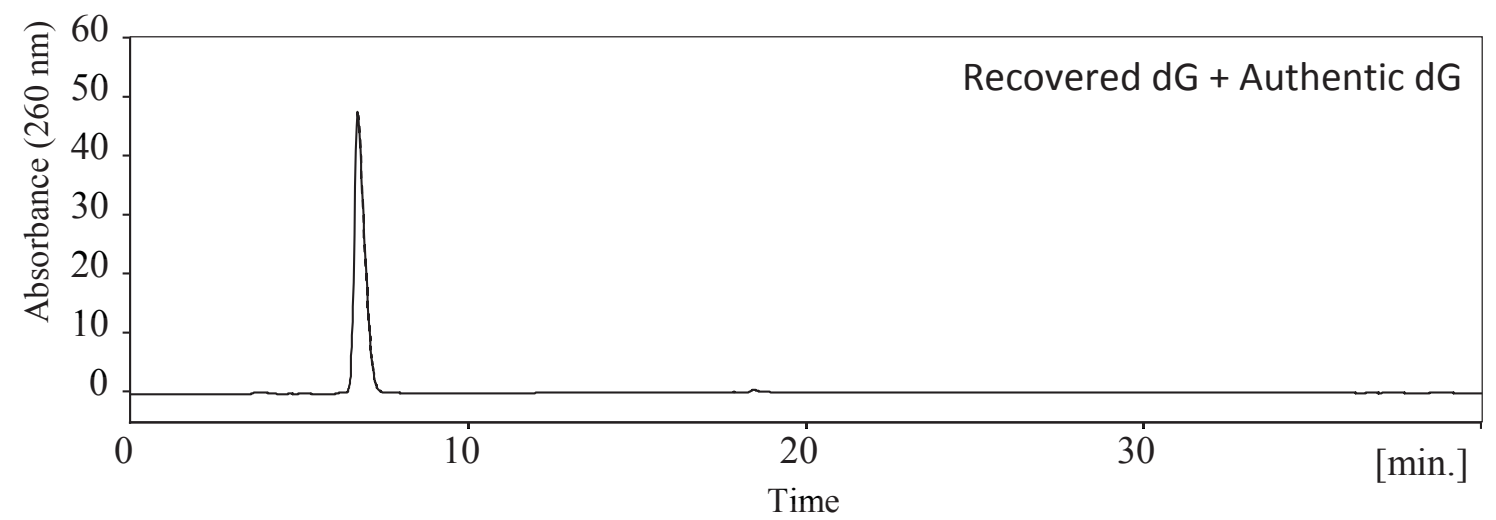

Figure 3.8. Co-injection HPLC profile of recovered guanosine and authentic guanosine

The co-injection HPLC profile of recovered guanosine and authentic 8-oxoguanosine was also generated using $10 \mu \mathrm{L}$ of each solution. Two peaks were observed, 
which indicated that the recovered guanosine was not oxidized to 8-oxo-guanosine (Figure 3.9).

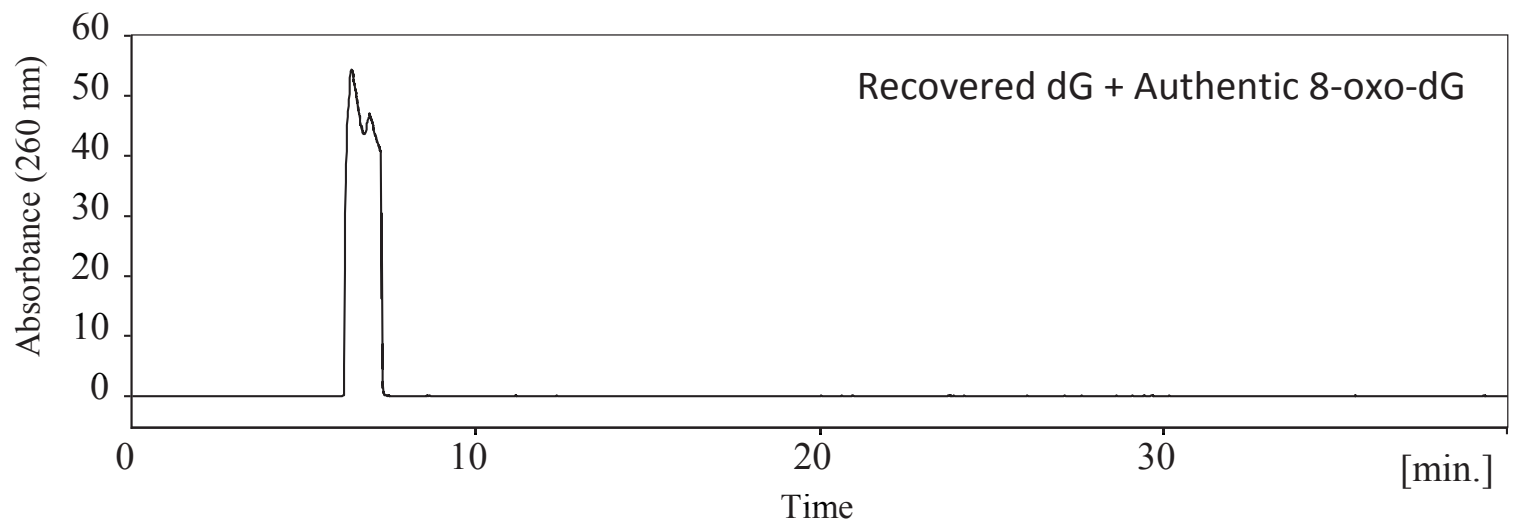

Figure 3.9. Co-injection HPLC profile of recovered guanosine and authentic 8-oxoguanosine

The other three nucleosides, adenosine (dA), thymidine (dT) and cytidine (dC), are more soluble in water, and the procedures for testing their stability under radical conditions were slightly different from that for $\mathrm{dG}$. The one for $\mathrm{dA}$ is described. Adenosine $(5.0 \mathrm{mg})$ was charged into a 2-necked round bottomed flask. A solution was formed after stirring with gentle heating with heat gun. To the solution, a dimethylacrylamide solution $[250 \mu \mathrm{L}$; $N, N$-dimethylacrylamide $1.69 \mathrm{M}, N, N^{\prime}$ - methylenebis(acrylamide) $\left.16.9 \mathrm{mM}\right]$ was added. The flask was flushed with nitrogen for $\sim 3$ min. $\left(\mathrm{NH}_{4}\right)_{2} \mathrm{~S}_{2} \mathrm{O}_{8}$ solution $(10 \%, 5 \mu \mathrm{L})$ and TMEDA $(5 \mu \mathrm{L})$ were added, and the mixture was gently stirred at $\mathrm{rt}$. The polymerization was allowed to proceed for $1 \mathrm{~h}$. Water $(2 \mathrm{~mL})$ was added to extract $\mathrm{dA}$ ( $\mathrm{rt}, 12 \mathrm{~h}$, gentle stirring). The extract was removed. The gel was further extracted with water ( $\mathrm{rt}, 3 \mathrm{~h}$, gentle stirring, $2 \mathrm{~mL} \times 2$ ). All extracts were combined and evaporated to dryness in a SpeedVac concentrator. The residue was dissolved in $10 \mathrm{~mL}$ water, $20 \mu \mathrm{L}$ were injected into HPLC 
(eluting conditions described in the general experimental section were used) to generate HPLC profile Recovered $d A$ (Figure 3.10). A single peak was observed.

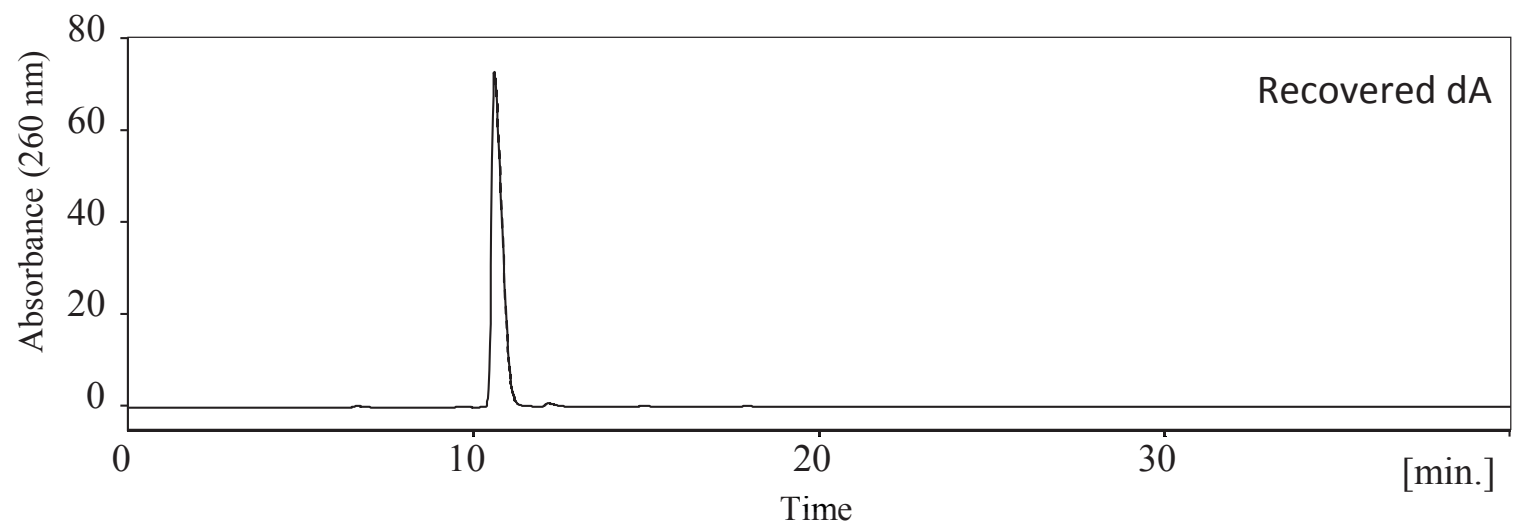

Figure 3.10. HPLC profile of recovered adenosine

The authentic dA $(5.0 \mathrm{mg})$ was dissolved in $10 \mathrm{~mL}$ water, $20 \mu \mathrm{L}$ were injected into HPLC to generate HPLC profile Authentic $d A$ (Figure 3.11).

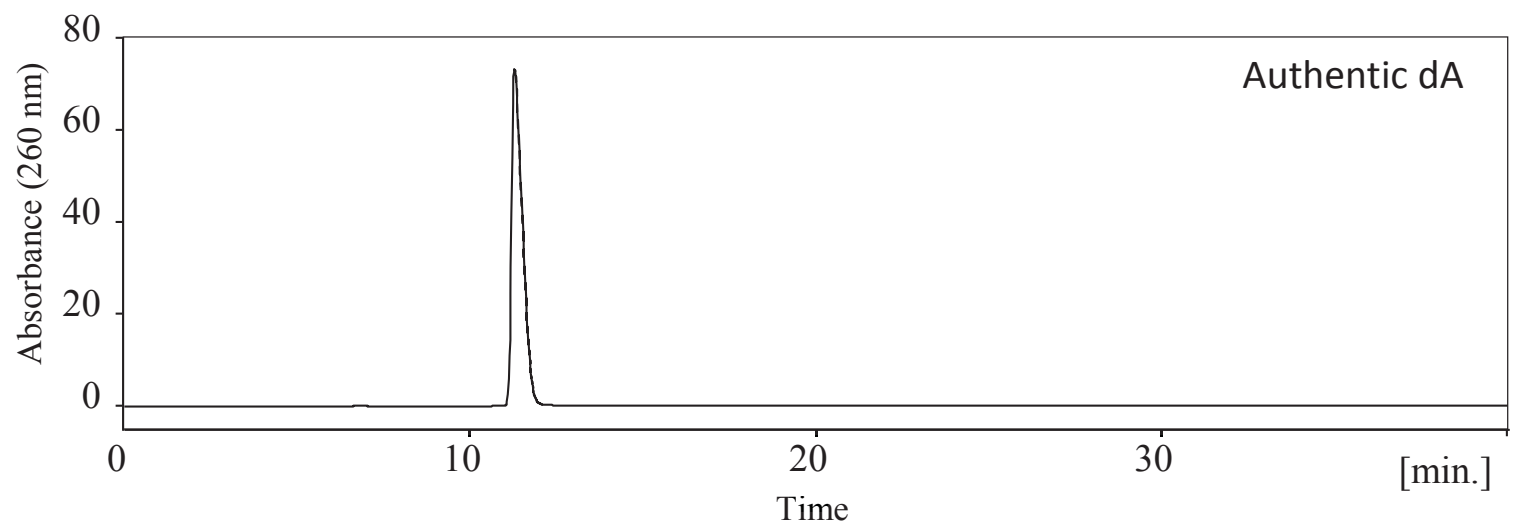

Figure 3.11. HPLC profile of authentic adenosine 
The co-injection HPLC profile Recovered $d A+$ Authentic $d A$ (Figure 3.12) was generated using $10 \mu \mathrm{L}$ of each solution. A single peak was observed, which indicated that they are identical.

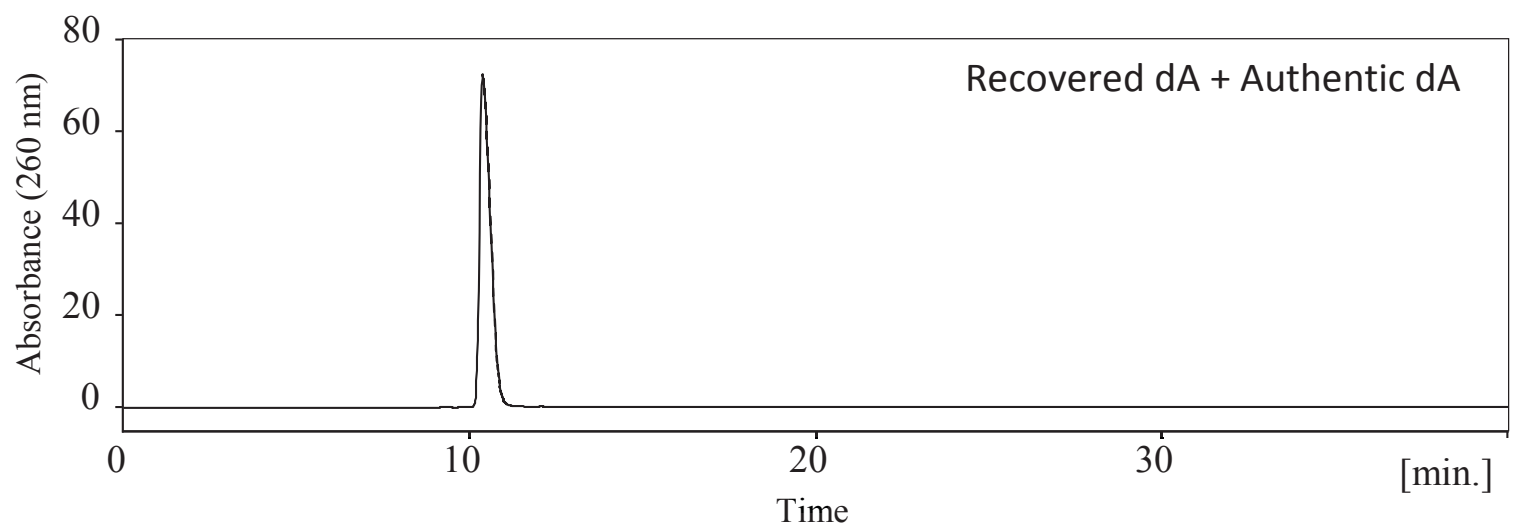

Figure 3.12. Co-injection HPLC profile of recovered adenosine and authentic adenosine

The nucleosides thymidine $(\mathrm{dT})$ and cytidine $(\mathrm{dC})$ were subjected into the same radical polymerization conditions, respectively. The recovered nucleosides were dissolved in $5 \mathrm{~mL}$ water, $20 \mu \mathrm{L}$ were injected into HPLC to generate profiles Recovered $d T$ (Figure 3.13) and Recovered $d C$ (Figure 3.14), respectively.

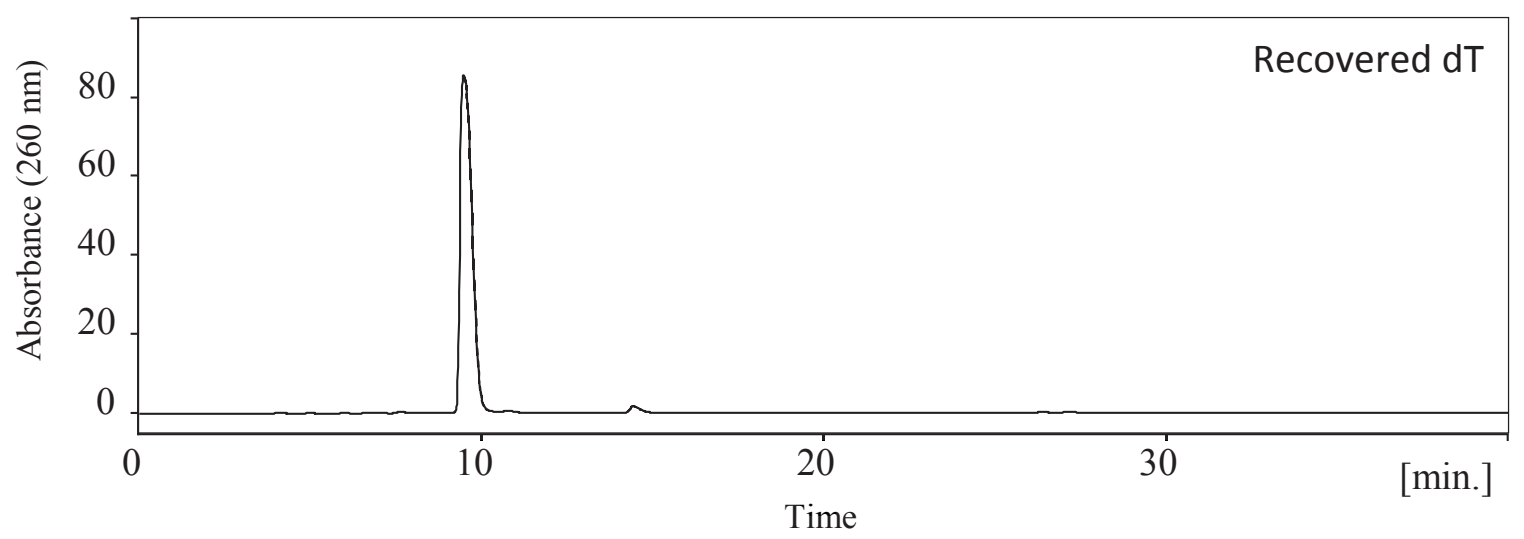

Figure 3.13. HPLC profile of recovered thymidine 


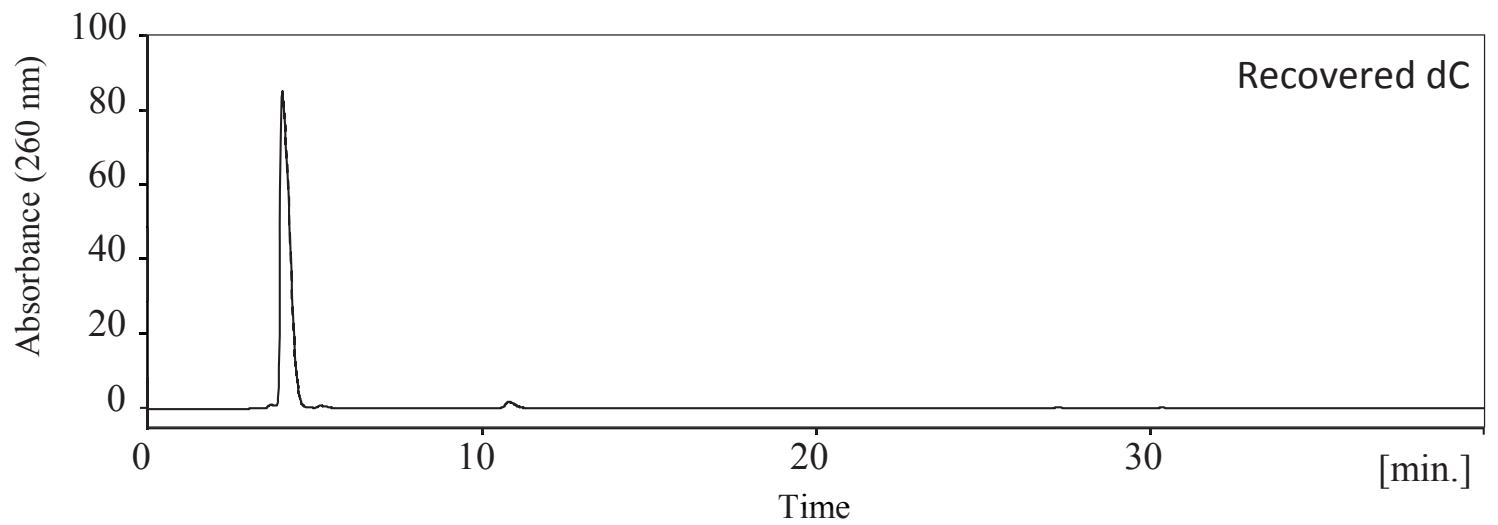

Figure 3.14. HPLC profile of recovered cytidine

The authentic $\mathrm{dT}(5.0 \mathrm{mg})$ and authentic $\mathrm{dC}(5.0 \mathrm{mg})$ were dissolved in $5 \mathrm{~mL}$ water, $20 \mu \mathrm{L}$ were injected into HPLC to generate profiles Authentic $d T$ (Figure 3.15) and Authentic dC (Figure 3.16), respectively.

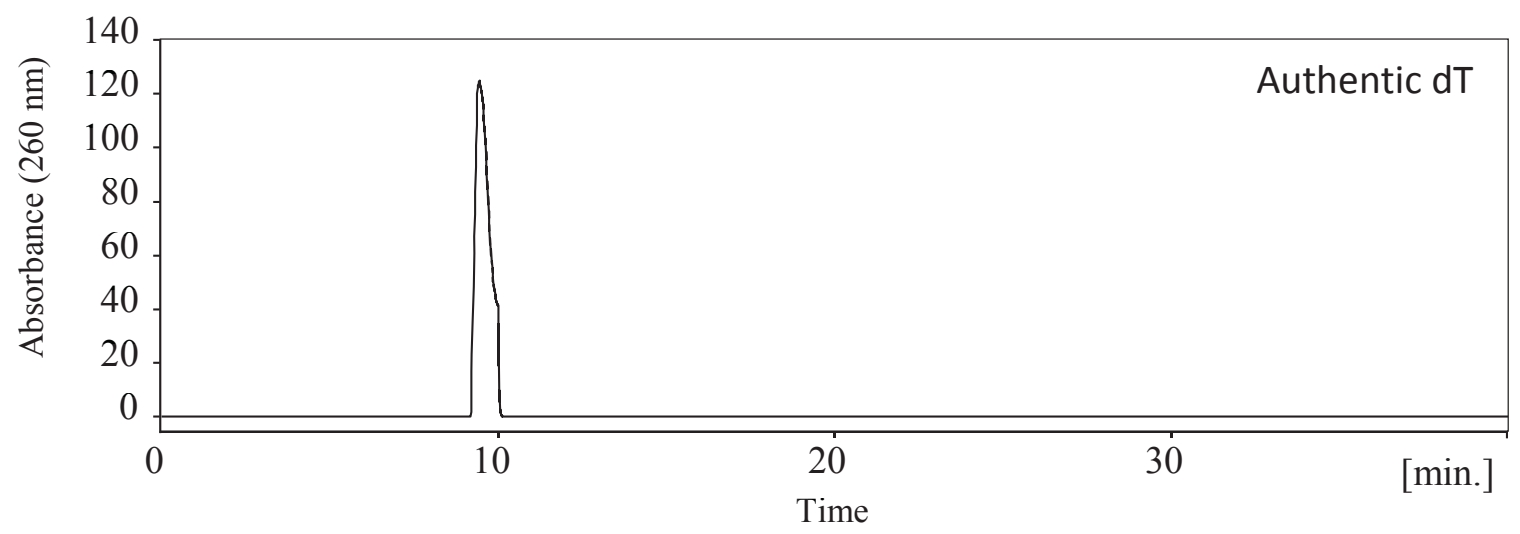

Figure 3.15. HPLC profile of authentic thymidine 


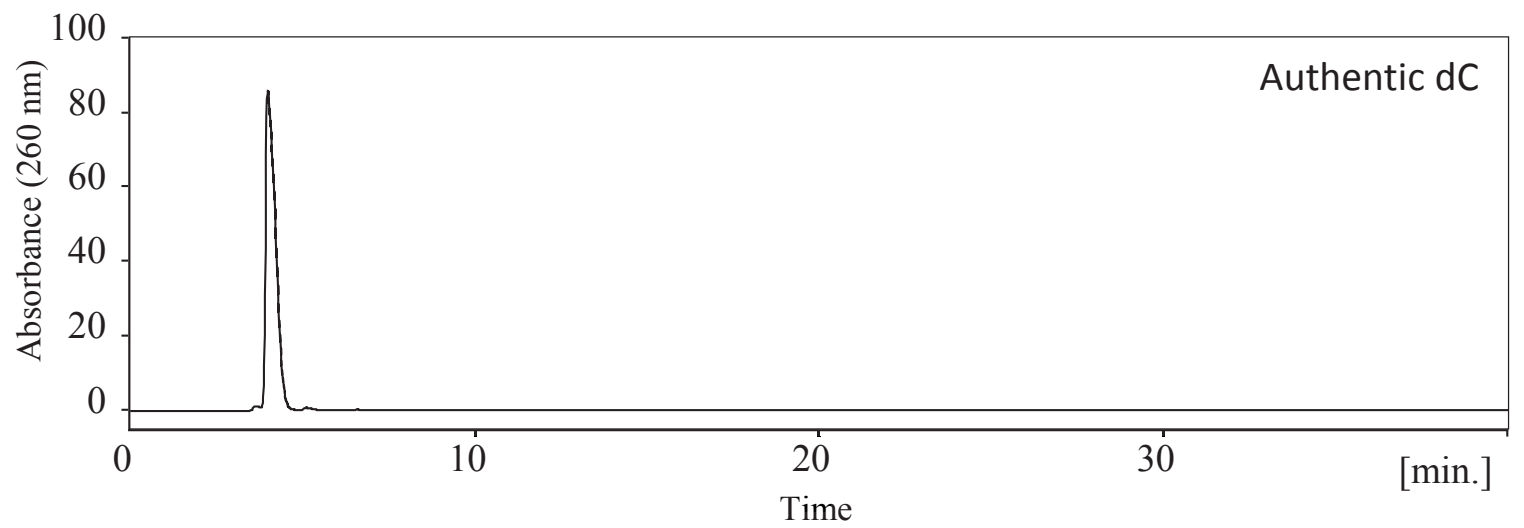

Figure 3.16. HPLC profile of authentic cytidine

The co-injection HPLC profiles Recovered $d T+$ Authentic $d T$ (Figure 3.17) and Recovered $d C+$ Authentic $d C$ (Figure 3.18) were generated using $10 \mu \mathrm{L}$ of each solution. Single peaks were observed, which indicated that the nucleosides were stable under the radical polymerization conditions.

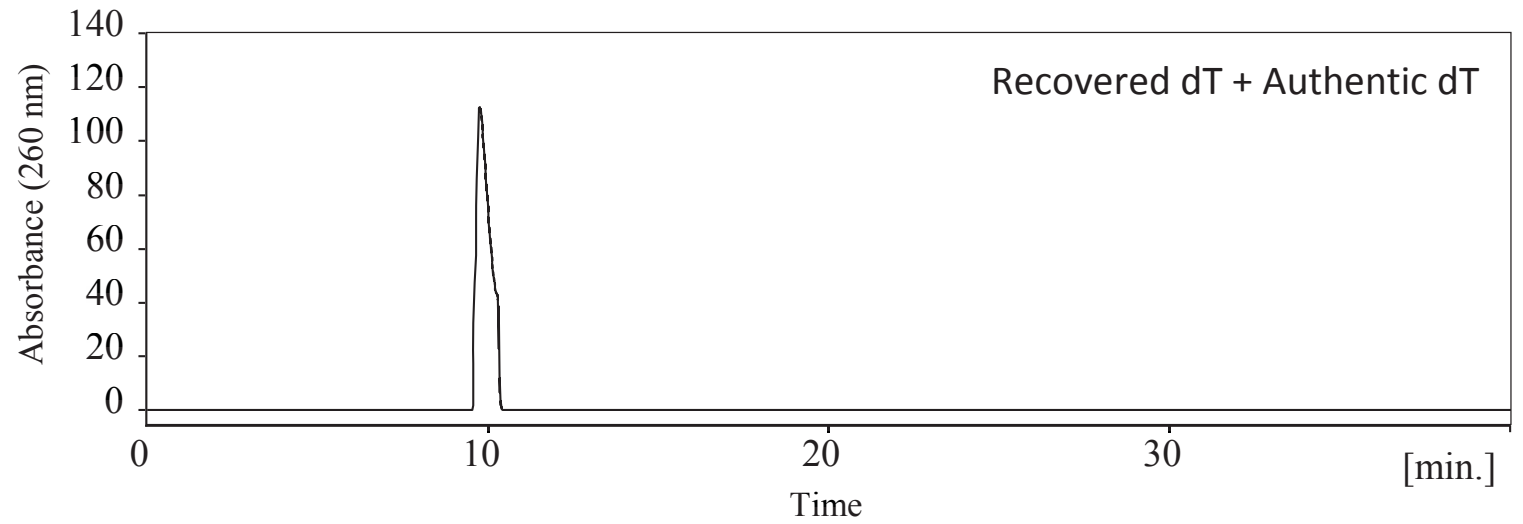

Figure 3.17. Co-injection HPLC profile of recovered thymidine and authentic thymidine 


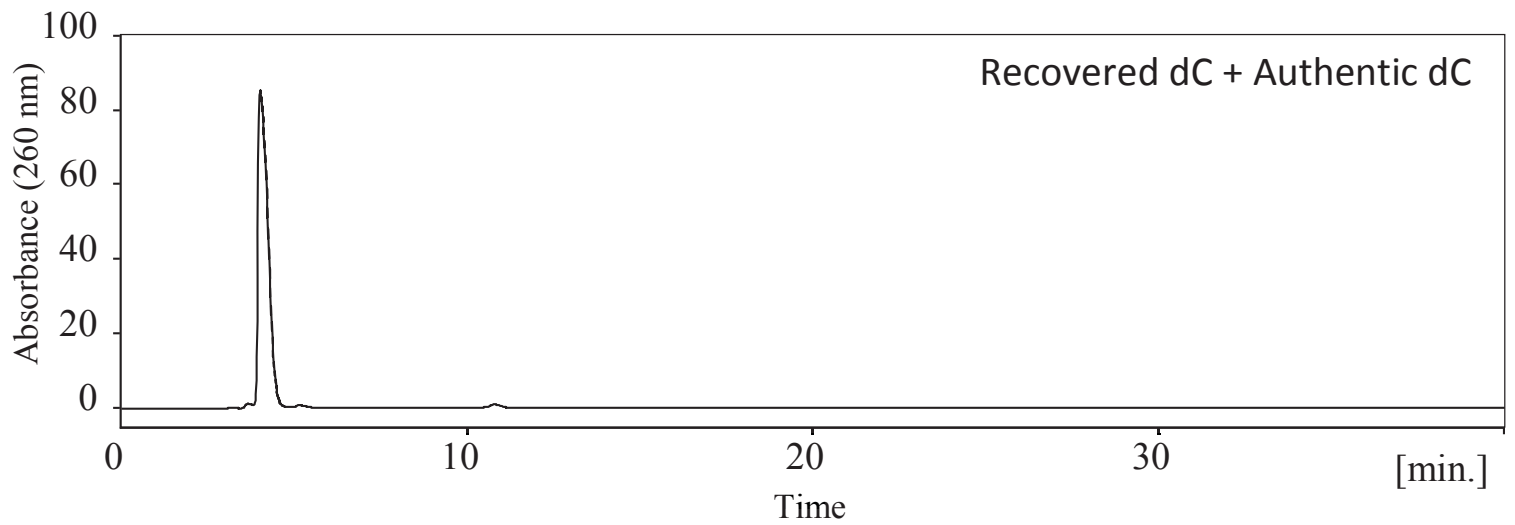

Figure 3.18. Co-injection HPLC profile of recovered cytidine and authentic cytidine 


\section{Acknowledgments}

Financial support from the U.S. NSF (CHE-0647129), Michigan Universities Commercialization Initiative, MTU Chemistry Department, MTU Biotech Research Center, and The Royal Thai Government Scholarship (S.F.); the assistance from P. P. Rao (preliminary synthetic studies of 3.1), Mr. Jerry L. Lutz (NMR), Mr. Shane Crist (computation), and Mr. Dean W. Seppala (electronics); and an NSF equipment grant (CHE9512445) are all gratefully acknowledged. 


\section{References and Notes}

1. (a) Hau, P.; Jachimczak, P.; Bogdahn, U., Treatment of malignant gliomas with TGF- $\beta 2$ antisense oligonucleotides. Expert Review of Anticancer Therapy 2009, 9 (11), 1663-1674; (b) Séguin, R. M.; Ferrari, N., Emerging oligonucleotide therapies for asthma and chronic obstructive pulmonary disease. Expert Opinion on Investigational Drugs 2009, 18 (10), 1505-1517; (c) Richardt-Pargmann, D.; Vollmer, J., Stimulation of the Immune System by Therapeutic Antisense Oligodeoxynucleotides and Small Interfering RNAs via Nucleic Acid Receptors. Annals of the New York Academy of Sciences 2009, 1175 (1), 4054; (d) Akhtar, S., Oral delivery of siRNA and antisense oligonucleotides. Journal of Drug Targeting 2009, 17 (7), 491-495; (e) Zhao, X.; Pan, F.; Holt, C. M.; Lewis, A. L.; Lu, J. R., Controlled delivery of antisense oligonucleotides: a brief review of current strategies. Expert Opinion on Drug Delivery 2009, 6 (7), 673-686.

2. Schulte, M.; Lühring, N.; Keil, A.; Sanghvi, Y. S., Purification of DMT-On Oligonucleotide by Simulated Moving-Bed (SMB) Chromatography. Organic Process Research \& Development 2005, 9 (2), 212-215.

3. Examples: (a) Beaucage, S. L.; Bergstrom, D. E.; Glick, G. D.; Jones, R. A. Current Protocols in Nucleic Acid Chemistry, ed. John Wiley \& Sons, New York 2000; (b) Fang, S.; Bergstrom, D. E., Reversible 5'-end biotinylation and affinity purification of synthetic RNA. Tetrahedron Letters 2004, 45 (43), 7987-7990; (c) Fang, S.; Bergstrom, D. E., Fluoride-cleavable biotinylation phosphoramidite for 5'-end-labeling and affinity purification of synthetic oligonucleotides. Nucleic Acids Research 2003, 31 (2), 708-715; (d) Fang, S.; Bergstrom, D. E., Reversible Biotinylation Phosphoramidite for 5'-EndLabeling, Phosphorylation, and Affinity Purification of Synthetic Oligonucleotides. 
Bioconjugate Chemistry 2002, 14 (1), 80-85; (e) Sproat, B. S.; Rupp, T.; Menhardt, N.; Keane, D.; Beijer, B., Fast and simple purification of chemically modified hammerhead ribozymes using a lipophilic capture tag. Nucleic Acids Research 1999, 27 (8), 1950-1955; (f) Pearson, W. H.; Berry, D. A.; Stoy, P.; Jung, K.-Y.; Sercel, A. D., Fluorous Affinity Purification of Oligonucleotides. The Journal of Organic Chemistry 2005, 70 (18), 71147122; (g) Beller, C.; Bannwarth, W., Noncovalent Attachment of Nucleotides by FluorousFluorous Interactions: Application to a Simple Purification Principle for Synthetic DNA Fragments. Helvetica Chimica Acta 2005, 88 (1), 171-179.

4. $\quad$ Fang, S. U.S. Patent pending US20080081902.

5. Tang, W.; Fang, S., Mono-acylation of symmetric diamines in the presence of water. Tetrahedron Letters 2008, 49 (41), 6003-6006.

6. The diisopropyl silyl acetal linker can also survive normal ODN cleavage and deprotection conditions such as $\mathrm{NH}_{4} \mathrm{OH}(29 \%) / \mathrm{H}_{2} \mathrm{NMe}(40 \%)(1: 1), 65{ }^{\circ} \mathrm{C}, 30 \mathrm{~min}$. When these conditions are used, the 2-cyanoethyl phosphoramidites benzoyl-dA, isobuyryl-dG, acetyl-dC, and dT can be used for the synthesis. See ref $3 \mathrm{c}$.

7. Sanghvi, Y. S.; Schulte, M., Therapeutic oligonucleotides: the state-of-the-art in purification technologies. Current Opinion in Drug Discovery and Development 2004, 7, 765-776.

8. Pieken, W.; Wolter, A.; Leuck, M. U.S. Patent US20030195351 A1, 2003.

9. Montanari, V.; Kumar, K., Just Add Water: A New Fluorous Capping Reagent for Facile Purification of Peptides Synthesized on the Solid Phase. Journal of the American Chemical Society 2004, 126 (31), 9528-9529. 
10. Palmacci, E. R.; Hewitt, M. C.; Seeberger, P. H., “Cap-Tag”-Novel Methods for the Rapid Purification of Oligosaccharides Prepared by Automated Solid-Phase Synthesis. Angewandte Chemie International Edition 2001, 40 (23), 4433-4437.

11. Yus, M.; Torregrosa, R.; Pastor, I., Masked $\omega$-Lithio Ester Enolates: Synthetic Applications. Molecules 2004, 9 (5), 330-348.

12. Hölemann, A.; Reissig, H.-U., Synthesis of New Furan Derivatives and 4Hydroxy Aldehydes from 4-Hydroxy 1-Enol Ethers. Synthesis 2004, 2004 (12), 1963 1970.

13. Pastor, I. M.; Yus, M., Masked $\beta$-, $\gamma$ - and $\delta$-lithium ester enolates: useful reagents in organic synthesis. Tetrahedron Letters 2001, 42 (6), 1029-1032.

14. Pieles, U.; Zürcher, W.; Schär, M.; Moser, H., Matrix-assisted laser desorption ionization time-of-flight mass spectrometry: a powerful tool for the mass and sequence analysis of natural and modified oligonucleotides. Nucleic Acids Research 1993, 21 (14), 3191-3196. 


\title{
Chapter 4
}

\section{Synthetic Oligodeoxynucleotide Purification by Polymerization}

\author{
of Failure Sequences*
}

Shiyue Fang, ${ }^{a}$ Suntara Fueangfung, ${ }^{a}$ Xi Lin, ${ }^{a}$ Xiang Zhang, ${ }^{a}$ Wenpeng Mai, ${ }^{a}$ Lanrong $\mathrm{Bi}^{a}{ }^{a}$ and Sarah A. Green ${ }^{a}$

${ }^{a}$ Department of Chemistry, Michigan Technological University, 1400 Townsend Drive, Houghton, MI 49931-1295 USA

Email: shifang@mtu.edu

*This material contained in this chapter was previously published in Chemical Communications, 2011, 47 (4), 1345-1347 -- Reproduced by permission of The Royal Society of Chemistry. See Appendix D. Permission from publishers. 


\begin{abstract}
Synthetic oligodeoxynucleotide is purified by capping failure sequences with an acrylated phosphoramidite followed by polymerization and product extraction. The method is suitable for large scale oligonucleotide drug purification.
\end{abstract}




\subsection{Introduction}

For most applications of synthetic oligonucleotides (ONs) such as in PCR, only submicromolar quantities are needed. These amounts of ONs can be synthesized using automated solid phase synthesis and purified with polyacrylamide gel electrophoresis or HPLC with reasonable costs. ${ }^{1}$ In recent years, recognition of the ability of ONs to selectively silence gene expression, and thus potentially treat a wide range of human diseases, demands quantities of pure ONs at the kilogram to metric ton scales. ${ }^{2}$ Synthesis at this level is now possible using solid phase synthesis and phosphoramiditechemistry. ${ }^{3}$ Besides the desired full-length sequence, the crude ON contains impurities, which include truncated failure sequences generated in each synthetic cycle due to incomplete coupling, small molecules resulting from protecting groups, and minute quantities of deletion sequences from incomplete detritylation and incomplete capping, and addition sequences from premature detritylation before and during coupling.

For a typical synthesis of a 20-mer oligodeoxynucleotide (ODN) on a controlled pore glass (CPG), the failure sequences constitute $30-60 \%$ of the total ODN content depending on the scale of synthesis. ${ }^{4}$ Because these impurities have the same physical properties as the full-length ODN, they are difficult to remove. The most powerful polyacrylamide gel electrophoresis (PAGE) can efficiently separate them, but this method cannot be scaled up. HPLC methods, especially trityl-on reverse phase (RP) and anion exchange HPLC, have been adapted to purify large scale ONs, which removes impurities including failure sequences. ${ }^{5}$ However, these methods require expensive instrument and preparative column. In addition, large volumes of solvent are needed, which must be 
subsequently evaporated. As a result, the method is very expensive for large scale purification.

Several other methods have also been developed for the removal of failure sequences including biotin-avidin enabled affinity extraction, ${ }^{6}$ fluorous affinity extraction,, 4 hydrophobic RP chromatography, ${ }^{8}$ and reaction-based solid phase extraction. ${ }^{9}$ All these methods are still expensive for large scale purification as we discussed in a previous paper. $^{10}$

Unlike the removal of failure sequences, the separation of small molecule contaminants can be easily achieved by known methods such as simple $n$-BuOH precipitation. ${ }^{11}$ Other ON-based impurities such as deletion and addition sequences are the most challenging to remove. Fortunately, they only exist in minute quantities. For large scale $\mathrm{ON}$ drug production, the best option is to adjust synthesis conditions to keep them at an acceptable level.

\subsection{Results and Discussion}

For separation of full-length ODN from failure sequences, we recently reported a method involving using a reversibly acrylated phosphoramidite to couple to the 50 -end of full-length sequence. Because the failure sequences were all capped with $\mathrm{Ac}_{2} \mathrm{O}$, only fulllength sequence had a polymerizable acrylamide group. The crude ODN was subjected to polymerization. The full-sequence was incorporated into a polymer, and the failure sequences were removed by washing. Cleaving the full-length ODN from the polymer gave a pure product. ${ }^{10}$ In this communication, we report an even more convenient method for ODN purification, which involves capping failure sequences with an acrylated 
phosphoramidite and incorporating them into a polymer. Using this method, full-length ODN can be simply extracted with water.

The acrylated phosphoramidite 4.1 (Figure 4.1) for this application was synthesized in two steps from 6-amino-1-hexanol with only one flash column chromatography purification. To prove the new purification concept, the 20-mer $4.4(0.2 \mathrm{mmol})$ was synthesized on CPG on a standard synthesizer. In the synthetic cycle (see appendix B), the steps for $\mathrm{Ac}_{2} \mathrm{O}$ capping in a standard cycle were removed. Instead, capping was achieved in added steps using a $0.2 \mathrm{M}$ acetonitrile solution of 4.1. This solution was placed on the synthesizer in a bottle typically employed for additional phosphoramidites besides the ones for natural nucleotides. The tetrazole solution $(0.45 \mathrm{M})$ used to activate 4.1 was from the same bottle used for the coupling steps. To ensure complete capping, $\mathbf{4 . 1}$ and the activator were delivered to the synthesis column four times instead of two times normally used in coupling. Because the failure sequences may locate at sites difficult for reagents to reach in $\mathrm{CPG}$, the oxidation of the newly formed phosphitetriesters was carried out three times. In the last cycle, detritylation was performed. Cleavage and deprotection were carried out with concentrated $\mathrm{NH}_{4} \mathrm{OH}$.

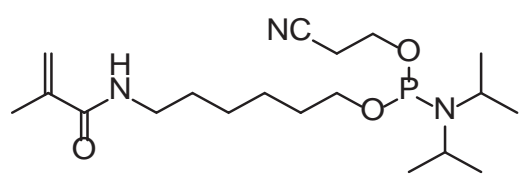

Figure 4.1. Structure of capping phosphoramidite 4.1 
The crude ODN was analyzed with RP HPLC. As shown in Figure 4.2, the fulllength sequence appeared at $\sim 19$ min. Normally, when $\mathrm{Ac}_{2} \mathrm{O}$ is used for capping, failure sequences have a shorter retention time than the full-length sequence. Here, due to the relatively more hydrophobic tag on $\mathbf{4 . 5}$, the order is reversed; and the failure sequences appeared at $\sim 20$ minutes. The small peaks at around 10, 28, and 56 min were probably due to small molecules from protecting groups. The tiny peaks, which could be hardly seen in a at the left of the full-length ODN, might be resulted from un-capped failure sequences, deletion sequences, or damaged sequences. ${ }^{12}$ According to our experience, from the relative intensity of peaks of the full-length ODN and the failure sequences, the modification of the synthetic cycle did not affect the efficiency of ODN synthesis.

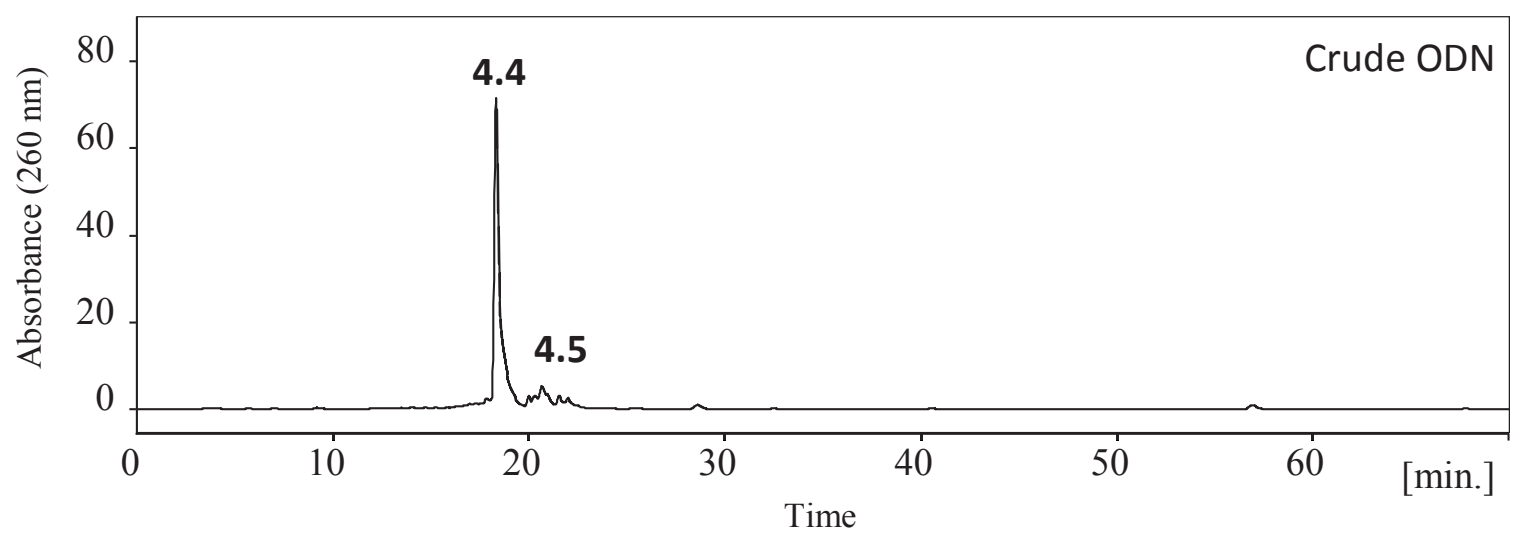

Figure 4.2. HPLC profile of crude ODN

The purification process is shown in Scheme 4.1. The crude ODN ( $\sim 50 \mathrm{nmol})$, which mainly contains the full-length sequence $\mathbf{4 . 4}$, failure sequences $\mathbf{4 . 5}$, and small molecules (not shown), was dissolved in $250 \mathrm{~mL}$ water. A solution of $\mathrm{N}, \mathrm{N}$ dimethylacrylamide and $N, N^{\prime}$-methylenebis(acrylamide) $(250 \mathrm{~mL})$ were added. The 
polymerization was initiated with $\left(\mathrm{NH}_{4}\right)_{2} \mathrm{~S}_{2} \mathrm{O}_{8}$ and TMEDA, and allowed to proceed at $\mathrm{rt}$ for $1 \mathrm{~h}$. The failure sequences 4.5 were incorporated into polymer 4.6. The full-length 4.4 and small molecules remained in the polymer matrix. Water was then added; and $\mathbf{4 . 4}$ and small molecules were extracted. The extract was analyzed with HPLC (Figure 4.3). To our surprise, four peaks instead of one at $\sim 19$ min appeared besides the weaker peaks from small molecules.

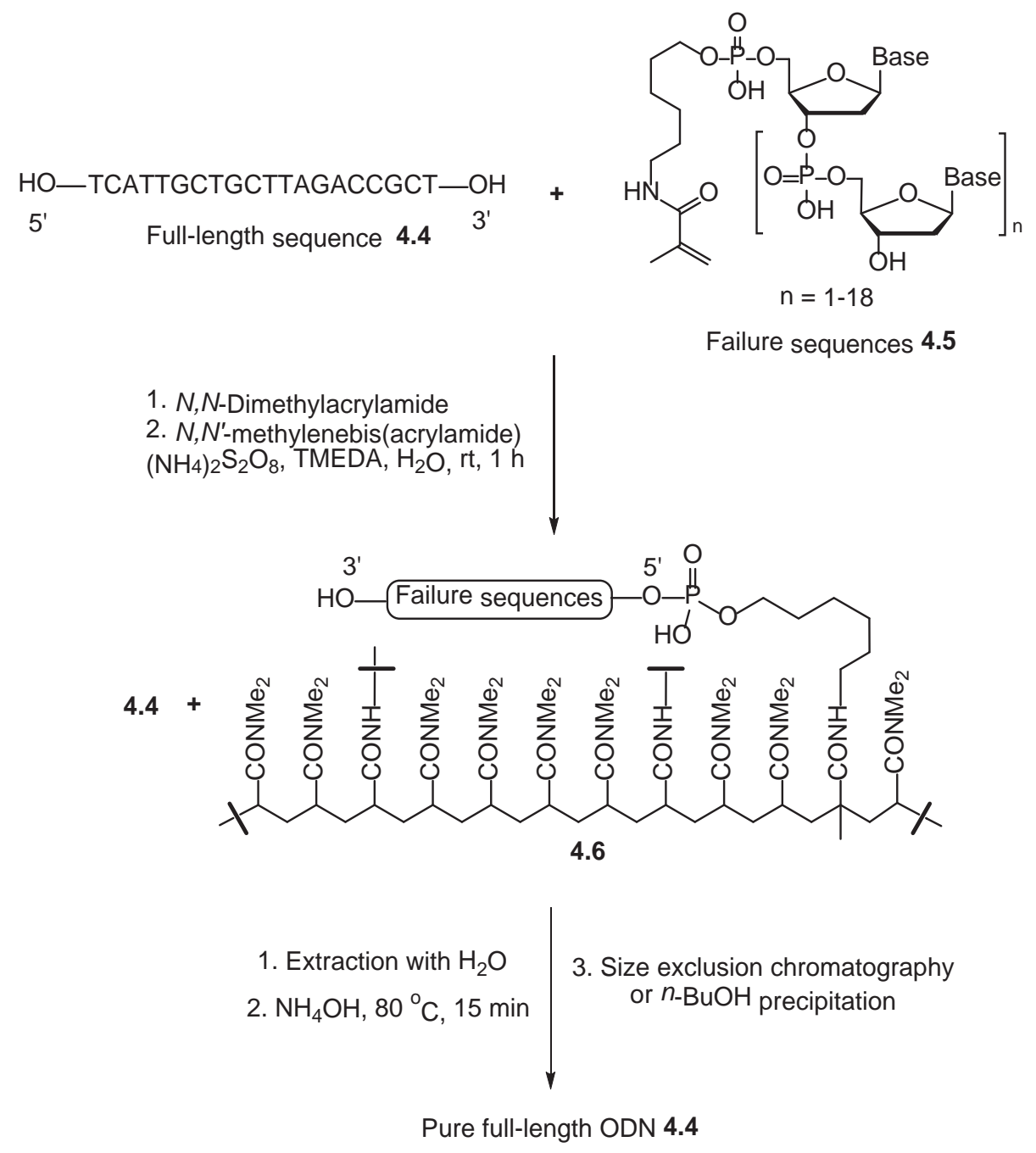

Scheme 4.1. Purification of ODN by polymerization of failure sequences 


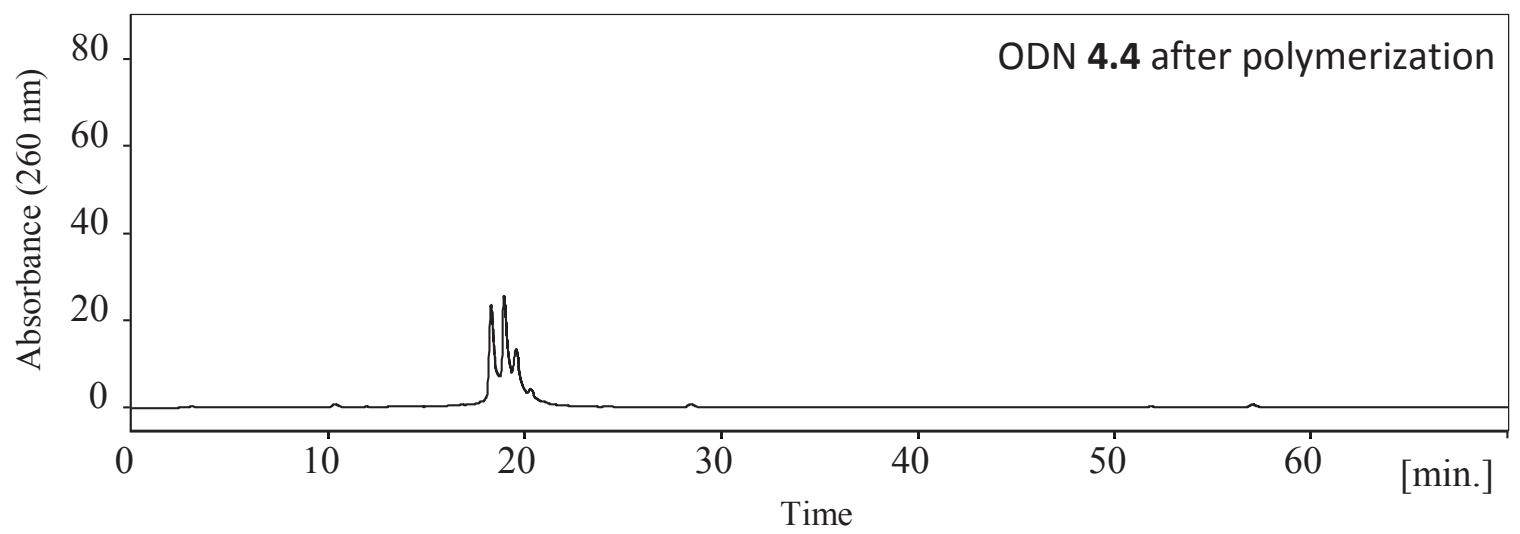

Figure 4.3. HPLC profile of pure ODN after polymerization

From our previous studies, ${ }^{10}$ we knew that ODN is stable under the radical polymerization conditions. We repeated the experiments for three times, and the HPLC profiles from all trials were identical. One possibility is that the nucleophilic sites of the full-length ODN reacted with acrylamide during polymerization through conjugate addition. ${ }^{13}$ If this is true, the adduct should not be stable and the reaction may be reversed to give unmodified ODN. Based on this hypothesis, the experiment was continued as planned. The extract was passed through a D-Salt dextran column (5 K MWCO), and analyzed with HPLC (Figure 4.4, trace a). As expected, the small molecules were removed. In addition, the four ODN peaks at $\sim 19$ min merged into three, which indeed supported our hypothesis of conjugate addition. To completely reverse the reaction, the ODN was heated in concentrated $\mathrm{NH}_{4} \mathrm{OH}$ at $80{ }^{\circ} \mathrm{C}$ for $15 \mathrm{~min},{ }^{14}$ and analyzed with HPLC. As shown in trace b (Figure 4.4), the remaining three peaks merged into one, and the ODN was pure. 

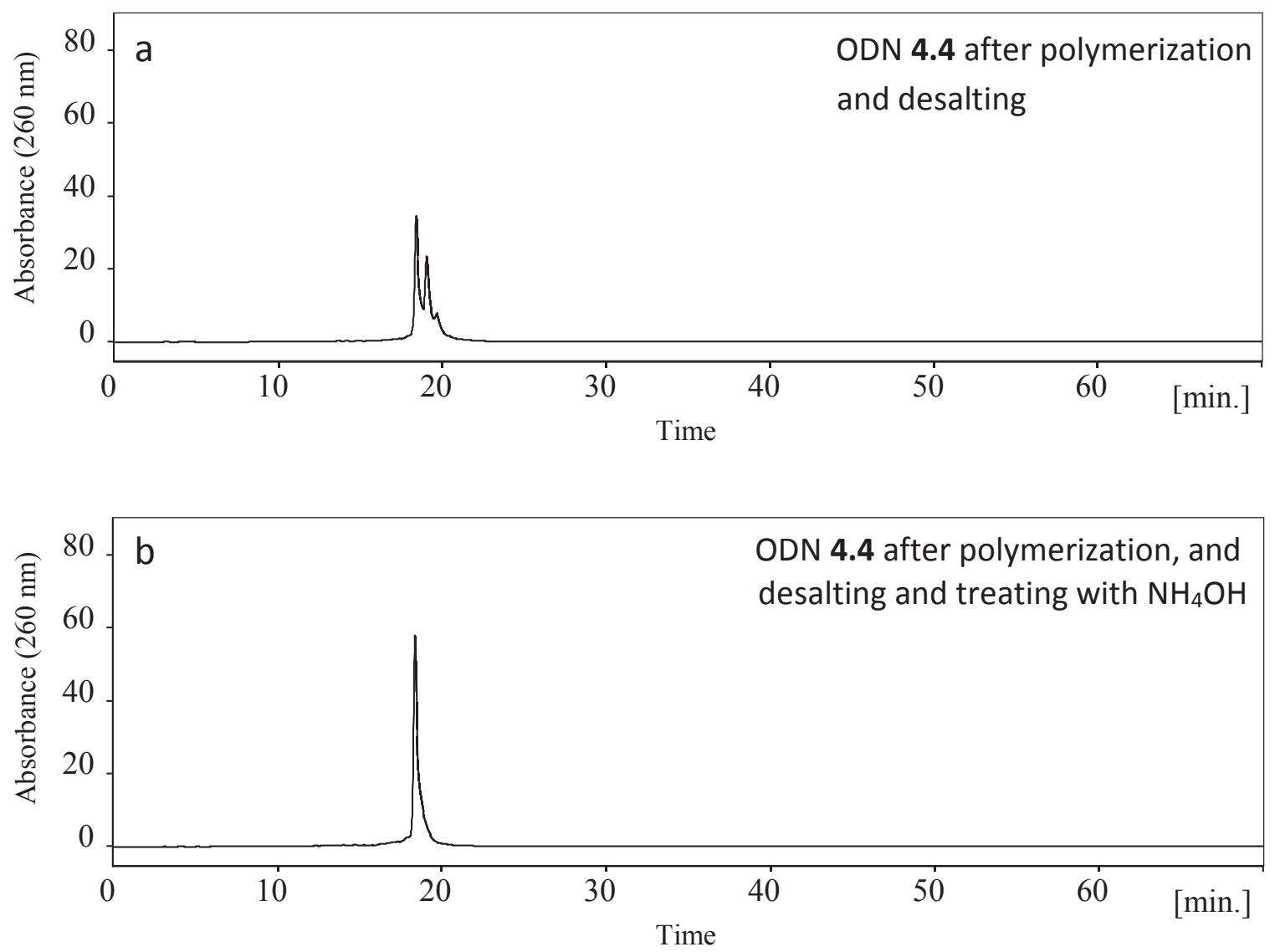

Figure 4.4. RP HPLC profiles of pure ODN 4.4 after (a) polymerization and desalting, (b) polymerization, desalting, and treating with $\mathrm{NH}_{4} \mathrm{OH}$ 
The identity of 4.4 was confirmed by co-injection with authentic sample. As shown in trace a (Figure 4.5), a single peak was observed. The trace for control (Figure 4.5, trace b) is also shown. MALDI-TOF analysis of $\mathbf{4 . 4}$ gave the correct molecular weight. The recovery yield of the purification process was determined to be $83 \%$ by comparing the area of the peak in trace $b$ of Figure 4.4 with the peak area in Figure 4.2 at $~ 19$ min.
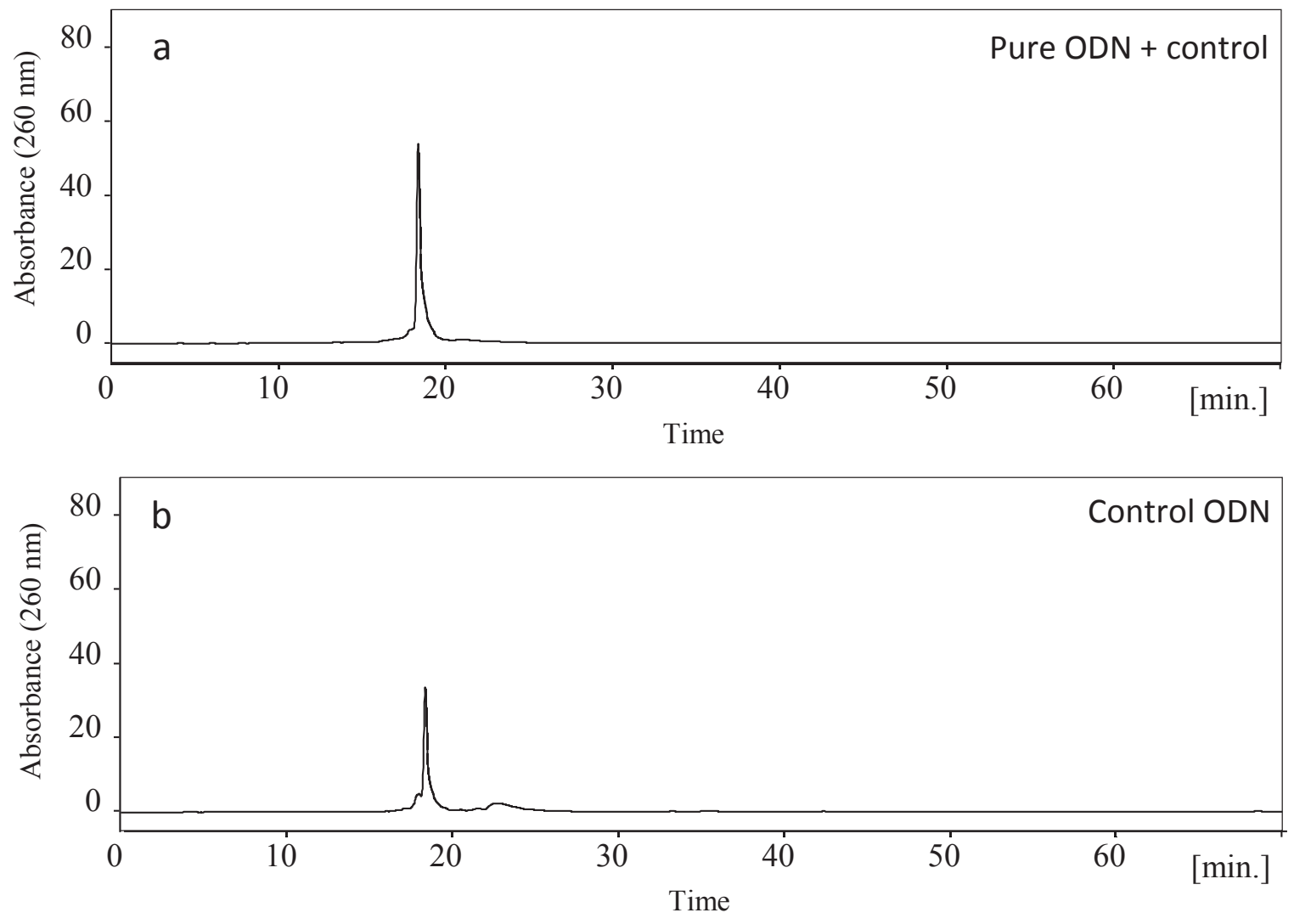

Figure 4.5. RP HPLC profiles of (a) co-injection of ODN 4.4 with control, (b) control ODN 
There are several known methods for removing small molecules from ODN including size exclusion chromatography, dialysis, and $\mathrm{EtOH}, 2-\mathrm{PrOH}$ or $n-\mathrm{BuOH}$ precipitation. ${ }^{11}$ Size exclusion chromatography, which we used in our initial studies, is easy to perform on small scales, but expensive on large scales. The $n-\mathrm{BuOH}$ precipitation method is simple, has high recovery yield, and can be easily scaled up. Therefore, we repeated the entire purification process including polymerization, extraction, and treating with concentrated $\mathrm{NH}_{4} \mathrm{OH}$. However, size exclusion chromatography was omitted. Instead, after treating with concentrated $\mathrm{NH}_{4} \mathrm{OH}, n-\mathrm{BuOH}$ was added. The ODN precipitated, while the small molecules remained in the supernatant. HPLC analysis showed that the ODN was pure (Figure 4.6). ${ }^{15}$ The recovery yield of the procedure was determined to be $85 \%$ by comparing the peak area in Figure 4.6 with the peak area in Figure 4.2 at $\sim 19$ min.

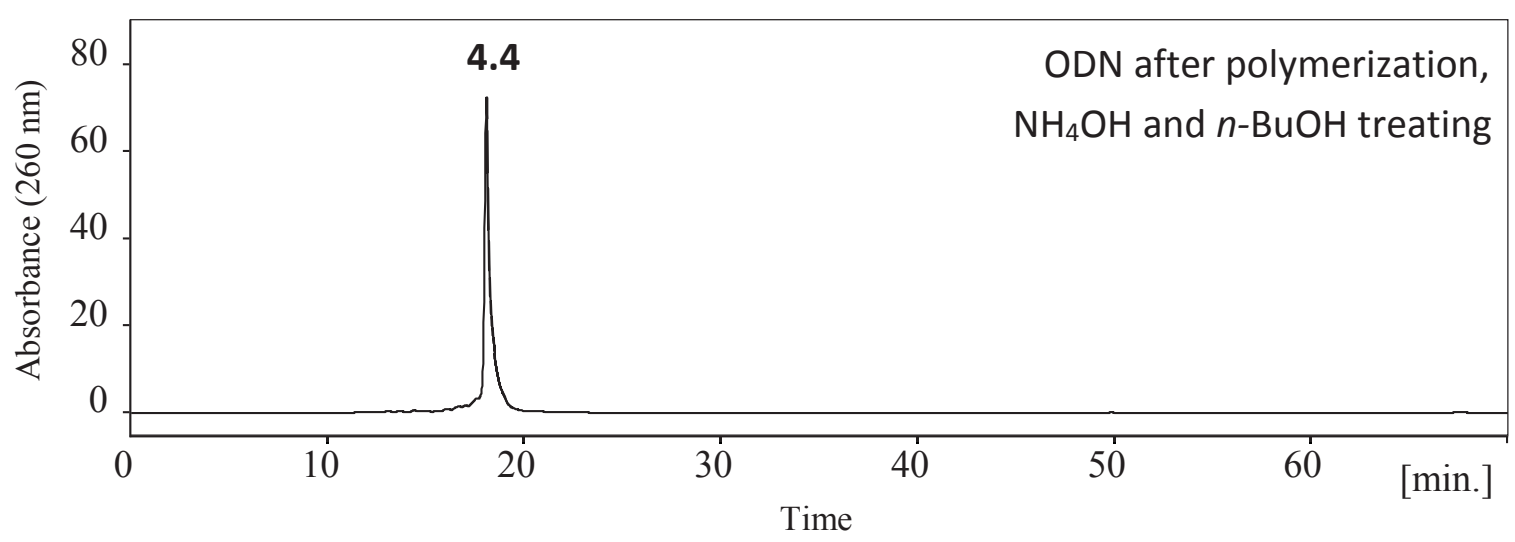

Figure 4.6. RP HPLC profiles of ODN 4.4 after polymerization, $\mathrm{NH}_{4} \mathrm{OH}$, and $n-\mathrm{BuOH}$ precipitation 
Phosphoramidite 4.1 is stable under nitrogen at $\sim 20^{\circ} \mathrm{C}$ for at least three weeks. We also put its $0.2 \mathrm{M}$ acetonitrile solution on DNA synthesizer for one week, no reduction of the capping efficiency was observed. Based on this information, 4.1 or its modified version could be made commercially available. Once this is realized, ODN purification, on large and small scales, can be simply achieved through the following steps: (1) synthesize ODN on a standard synthesizer using 4.1 as the capping agent. (2) Add reagents to polymerize failure sequences. (3) Extract full-length sequence by water. (4) Treat with concentrated $\mathrm{NH}_{4} \mathrm{OH}$ briefly. (5) Add $n$-BuOH to precipitate pure ODN. This purification method does not need any chromatography, which requires expensive instrument and large volumes of solvent (solvent to ODN mass ratio can be as high as $10^{5}$ ). Instead, purification is achieved by simple operations such as shaking and extraction. Using this method, the waste to product mass ratio (defined as the weight of polymerization reagents divided by that of ODN) in the purification procedure can be less than $10^{2}$. As a result, the method is suitable for large scale ODN drug purification.

The current ODN purification method and the method we reported earlier complement each other. In the earlier method, ${ }^{10}$ purification is achieved by polymerization of full-length sequence followed by washing, cleavage and extraction. That method only requires the polymerizable phosphoramidite in the last synthetic cycle, and the failure sequences are capped with less expensive $\mathrm{Ac}_{2} \mathrm{O}$. In addition, impurities such as the small molecules from protecting groups can also be removed in the washing step. Therefore that method is suitable for purification of long ODN (longer than 25-mer). For the current method, although the polymerizable phosphoramidite is needed in each synthetic cycle and impurities other than failure sequences have to be removed by $n$-BuOH precipitation, it has 
significant advantages. The polymerizable phosphoramidite is far less expensive, and there is no need to cleave ODN from polymer. These two features make the current method far more convenient and less expensive to execute. Therefore, this method is highly suitable for purification of short ODN (shorter than 25-mer) on any scale.

\subsection{Conclusion}

We have developed a new method for ODN purification. This method does not need any chromatography or expensive reagents, and purification is achieved by simple operations such as shaking and extraction. Therefore, it is suitable for purification of large scale ON drugs. Studies on extending this method to purify other synthetic oligomers such as peptides are underway.

\subsection{Experimental Section}

\section{General experimental}

ODNs were synthesized on a standard ABI 394 solid phase synthesizer. MALDITOF mass spectra were obtained on a Shimadzu Biotech Axima CFRplus spectrometer. HPLC was performed on a JASCO LC-2000Plus System: pump, PU-2089Plus Quaternary Gradient; detector UV-2075Plus. A C-18 reverse phase analytical column (5 $\mu \mathrm{m}$ diameter, $100 \AA, 250 \times 3.20 \mathrm{~mm}$ ) was used. Solvent A: $0.1 \mathrm{M}$ triethylammonium acetate, $5 \%$ acetonitrile. Solvent B: 90\% acetonitrile. All profiles were generated by detection of absorbance of ODN at $260 \mathrm{~nm}$ using the linear gradient solvent system: solvent B ( $0 \%$ 45\%) in solvent A over 60 min followed by solvent B (45\%-100\%) in solvent A over 20 min at a flow rate of $0.5 \mathrm{~mL} / \mathrm{min}$. Succinic ester linked DMTr-dT-lcaa-CPG (pore size 
$1000 \AA$ ) and 5'-DMTr, 2-cyanoethyl phosphoramidites acetyl-dC, Pac-dA, 4-isopropylPac-dG and dT were purchased from Glen Research, Inc. DSalt TM dextran desalting column (5K MWCO) was purchased from Pierce Biotechnology, Inc.

\section{Synthesis of phosphoramidite 4.1}
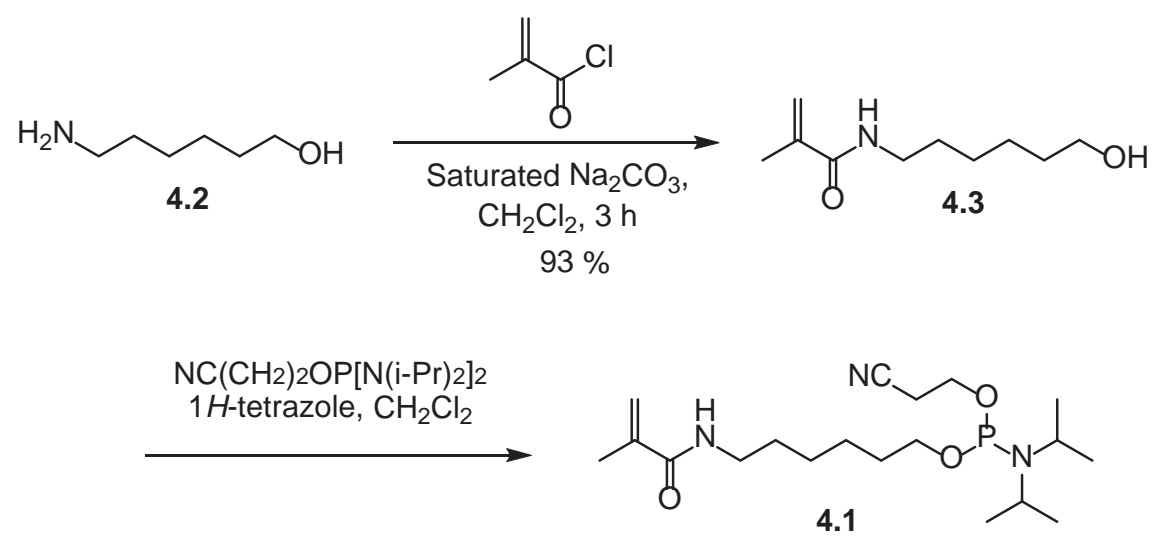

Compound 4.1 is known. ${ }^{16}$ We used a different method to prepare 4.3 , and only this step is described here. Compound $4.2(5.0 \mathrm{~g}, 42.7 \mathrm{mmol})$, saturated $\mathrm{Na}_{2} \mathrm{CO}_{3}$ solution (50 $\mathrm{mL})$ and $\mathrm{CH}_{2} \mathrm{Cl}_{2}(150 \mathrm{~mL})$ were charged into a round bottom flask, and cooled to $0{ }^{\circ} \mathrm{C}$. To the solution was added the solution of methacryloyl chloride (4.46 g, $42.7 \mathrm{mmol})$ in $\mathrm{CH}_{2} \mathrm{Cl}_{2}$ $(50 \mathrm{~mL})$ dropwise with efficient stirring via an addition funnel. After addition, the mixture was stirred at $\mathrm{rt}$ for $3 \mathrm{~h}$, and then transferred into a separation funnel. The organic layer was separated. The aqueous phase was extracted with $\mathrm{CH}_{2} \mathrm{Cl}_{2}$ for three times. The combined organic phase was dried over anhydrous $\mathrm{Na}_{2} \mathrm{SO}_{4}$, and filtered. The solution was concentrated to give a sticky liquid (not completely dry). Ether was added until white solids appeared, which was re-dissolved by adding $\mathrm{CH}_{2} \mathrm{Cl}_{2}$. To make the solution more dilute, hexanes and more $\mathrm{CH}_{2} \mathrm{Cl}_{2}$ were added. The solution was then put in a freezer $\left(-20^{\circ} \mathrm{C}\right)$ for 
$12 \mathrm{~h}$. Compound 4.3 was obtained as a colorless crystal: $7.33 \mathrm{~g}, 93 \%$ yield. If the compound melts at $\mathrm{rt}$, a second crystallization from $\mathrm{CH}_{2} \mathrm{Cl}_{2} /$ hexanes may be desirable.

\section{Synthesis of ODN 4.4}

\section{3' HO-TCGCCAGATTCGTCGTTAC-OH 5' (4.4)}

ODN 4.4 was synthesized on a standard ABI 394 solid phase synthesizer using standard phosphoramidite chemistry under UltraMild conditions on controlled pore glass (CPG, pore size $1000 \AA$ ) on a $0.2 \mu \mathrm{mol}$ scale. The succinyl ester linkage was used to anchor the ODN to CPG. The phosphoramidite monomers used were Pac-dA-CE, Ac-dC-CE, $i$ Pr-Pac-dGCEanddT-CE. A $0.2 \mathrm{M}$ solution of the polymerizable capping phosphoramidite 4.1 in acetonitrile was placed on the 5th bottle position, which is normally used for incorporating an additional base into ODN. The two bottles normally used to supply $\mathrm{Ac}_{2} \mathrm{O}$ capping reagents were empty. The synthesis was accomplished using the synthetic cycle shown in appendix B. In this synthesis, the capping failure sequences step was achieved using the polymerizable phosphoramidite 4.1 with $1 H$-tetrazole as the activator. The activator was from the same bottle that provided $1 H$-tetrazole for the coupling steps. To ensure complete capping, $\mathbf{4 . 1}$ and tetrazole were delivered to the synthesis column four times instead of two times normally used for standard nucleobase coupling (we found that capping two times were not enough for complete capping under the conditions we used. This can be seen in Figure B.7 of Appendix B), where the small peaks before the major full-length peak are un-capped failure sequences). Between each delivery, a waiting time of 15 seconds was applied. The oxidation of the phosphitetriesters between the capping agent and failure sequences was carried out for three times. In the last synthetic cycle, the 
DMTr group was removed. Cleavage and deprotection were carried out on the synthesizer with concentrated $\mathrm{NH}_{4} \mathrm{OH}(900 \mathrm{~min} \times 4)$ at rt. The $\mathrm{ODN}$ solution was distributed equally into four Eppendorf tubes $(1.5 \mathrm{~mL}$ ), and dried in a SpeedVac, separately (each portion contained $\sim 50 \mathrm{nmol}$ ODN, $P 1-P 4)$. $P 1$ was dissolved in $150 \mu \mathrm{L}$ water, $20 \mu \mathrm{L}(\sim 6.67 \mathrm{nmol})$ was injected into RP HPLC to generate trace in Figure 4.2. The full-length sequence appeared at $\sim 19$ minutes. The failure sequences were at $\sim 20$ minutes. The small peaks at around 10,28 , and 56 minutes were probably due to the small molecules from protecting groups. The very small peaks, which could be hardly seen in Figure 4.2 at the left of the full-length sequence, might be resulted from un-capped failure sequences, deletion sequences, or damaged sequences.

\section{Polymerization of failure sequences}

The remaining $130 \mu \mathrm{L}$ solution of $P 1$ was transferred into a 2-necked round bottom flask. The Eppendorf tube was washed with water $(50 \mu \mathrm{L} \times 3)$; the washes were also placed into the same flask. To the flask was added the pre-formed polymerization solution [250 $\mu \mathrm{L} ; N, N$-dimethylacrylamide $1.69 \mathrm{M}, N, N^{\prime}$-methylenebis(acrylamide) $16.9 \mathrm{mM}$; the solution could be stored at $-20^{\circ} \mathrm{C}$ in dark for 1 month]. The flask was flushed with nitrogen for 2 min with gentle stirring. $\left(\mathrm{NH}_{4}\right) \mathrm{S}_{2} \mathrm{O}_{8} \quad(10 \%, \quad 5 \quad \mu \mathrm{L})$ and $N, N, N^{\prime}, N^{\prime}-$ tetramethylethylenediamine (TMEDA, $5 \mu \mathrm{L}$ ) were added sequentially via pipettes under positive nitrogen pressure. The solution was stirred gently under nitrogen at rt. A gel, which was the ODN failure sequences-polyacrylamide conjugate 4.6, was formed within 30 min. The gel was allowed to stand for another 30 min to ensure completion of polymerization. 


\section{Extraction of full-length sequence}

To the gel, which was broken into several pieces, was added water $(200 \mu \mathrm{L})$. The mixture was stirred gently (shaking should be better) at $\mathrm{rt}$ for $3 \mathrm{~h}$. The supernatant was transferred into an Eppendorf tube. The gel was further extracted with water for two times (200 $\mu \mathrm{L}, \mathrm{rt}, 12 \mathrm{~h} ; 200 \mu \mathrm{L}, \mathrm{rt}, 3 \mathrm{~h})$. The supernatants were combined and evaporated into dryness. The ODN was dissolved into $130 \mu \mathrm{L}$ water; $20 \mu \mathrm{L}$ was injected into HPLC to generate trace in Figure 4.3. As shown, the failure sequences were removed, but the fulllength sequence appeared as four peaks. The small peaks resulted from small molecules from protecting groups also remained as expected.

\section{Size exclusion chromatography to remove small molecules}

A $10 \mathrm{~mL}$ D-Salt ${ }^{\mathrm{TM}}$ dextran desalting column (5K MWCO) was used. The column was first washed with water $(20 \mathrm{~mL})$. The remaining $110 \mu \mathrm{L}$ solution of ODN 4.4 was loaded to the top of the column. The Eppendorf tube was washed with water $(100 \mu \mathrm{L} \times 3)$, and the washes were also loaded to the column. The column was washed with $1.59 \mathrm{~mL}$ water. This first $2 \mathrm{~mL}$ eluent did not contain any ODN. The elution was continued and the next $5 \mathrm{~mL}$ was collected and evaporated to dryness. The residue was dissolved in $110 \mu \mathrm{L}$ water, $20 \mu \mathrm{L}$ was injected into HPLC to generate trace a, Figure 4.4. As shown, the small molecules from protecting groups were removed. The four peaks at around 19 min were merged into three peaks. The desalting column was recovered by washing with water (20 $\mathrm{mL}$ ), and was stored in $0.02 \% \mathrm{NaN}_{3}$ solution. 


\section{Treating with concentrated $\mathrm{NH}_{4} \mathrm{OH}$}

The remaining $90 \mu \mathrm{L}$ solution of ODN 4.4 was evaporated to dryness in an Eppendorf tube. Concentrated $\mathrm{NH}_{4} \mathrm{OH}(300 \mu \mathrm{L})$ was added. The solution was heated to 80 ${ }^{\circ} \mathrm{C}$ for 15 min in a sand bath. After evaporation to dryness, the residue was dissolved into

$90 \mu \mathrm{L}$ water, $20 \mu \mathrm{L}$ was injected into HPLC to generate trace b, Figure 4.4. As shown, only one peak is observed, and the ODN is pure. The recovery yield of the purification process (polymerization, extraction, size exclusion chromatography and $\mathrm{NH}_{4} \mathrm{OH}$ treatment) was determined to be $83 \%$ by comparing the area of the peak in trace b, Figure 4.4 with the area of the peak in Figure 4.1 at $\sim 19$ min.

\section{Identification of ODN 4.4}

The authentic ODN of $\mathbf{4 . 4}$ was synthesized using standard phosphoramidite chemistry on a $1 \mu \mathrm{mol}$ scale and purified with trityl-on RP HPLC at The Midland Certified Reagent Company, Inc. (Midland, TX, USA). MALDI-TOF spectrum of the authentic ODN was also obtained at the company showing correct molecular weight: calculated for $[\mathrm{M}-\mathrm{H}]^{-}$6057, found 6060. At Michigan Tech, the authentic sample was divided equally into 10 portions (each portion contains $\sim 100 \mathrm{nmol}$ ODN assuming the yield for the synthesis and purification was $100 \%$ ). One portion was dissolved in $150 \mu \mathrm{L}$ water, $20 \mu \mathrm{L}$ was injected into RP HPLC to generate trace a (Figure 4.5). To compare ODN 4.4 synthesized in our lab and purified using our catching failure sequences by polymerization technique with the authentic sample, $10 \mu \mathrm{L}$ of the solution used to generate trace a (Figure 4.5) and $10 \mu \mathrm{L}$ of the solution used to generate trace b (Figure 4.4) were mixed, and injected into RP HPLC to generate trace b (Figure 4.5). A single peak was observed showing the 
two were identical. ODN 4.4 was also characterized with MALDI-TOF: calcd for $[\mathrm{M}-\mathrm{H}]^{-}$ 6057, found: 6057 (See appendix B).

\section{ODN purification by polymerization of failure sequences and $n-\mathrm{BuOH}$ precipitation.}

The ODN 4.4 (P2, $50 \mathrm{nmol})$ was dissolved into $150 \mu \mathrm{L}$ water, $20 \mu \mathrm{L}$ was injected into HPLC, which gave a trace exactly the same as Figure 4.1. The remaining $130 \mu \mathrm{L}$ ODN solution was subjected into the procedure of polymerization of failure sequences and extraction of full-length sequence as described above. The size exclusion chromatography step was not performed. To the full-length sequence, which contained ODNs and small organic molecules from protecting groups, was added concentrated $\mathrm{NH}_{4} \mathrm{OH}(100 \mu \mathrm{L})$. The solution was vortexed shortly and then heated to $80^{\circ} \mathrm{C}$ for $30 \mathrm{~min}$. This converted the modified ODNs to the un-modified one. After cooling to $\mathrm{rt}, n$-BuOH $(1 \mathrm{~mL})$ was added. The mixture was vortexed for $30 \mathrm{sec}$ and then centrifuged at $14.5 \mathrm{~K}$ for $5 \mathrm{~min}$. The supernatant was removed. The residue was re-dissolved into $50 \mu \mathrm{L}$ water, $500 \mu \mathrm{L} n$-BuOH was added. Votexed and centrifuged again, and the supernatant was removed. This removed the small organic molecules resulted from deprotection. The ODN was dissolved in $130 \mu \mathrm{L}$ water, $20 \mu \mathrm{L}$ was injected into HPLC to generate Figure 4.6. As shown, the ODN is pure. The recovery yield of the procedure (polymerization, extraction, $\mathrm{NH}_{4} \mathrm{OH}$ treatment and $n$ - $\mathrm{BuOH}$ precipitation) was determined to be $85 \%$ by comparing the area of the peak in Figure 4.6 with the peak area in Figure 4.1 at $\sim 19$ min. 


\section{Acknowledgements}

Financial support from US NSF (CHE-0647129), Michigan Universities Commercialization Initiative, MTU Chemistry Department and Biotech Research Center, and The Royal Thai Government Scholarship (S. F.); the assistance from Mr. Jerry L. Lutz (NMR), Mr. Shane Crist (computation), and Mr. Dean W. Seppala (electronics) are gratefully acknowledged.

[Fang, S.; Fueangfung, S.; Lin, X.; Zhang, X.; Mai, W.; Bi, L.; Green, S. A., Synthetic oligodeoxynucleotide purification by polymerization of failure sequences. Chemical Communications 2011, 47 (4), 1345-1347.] - Reproduced by permission of The Royal Society of Chemistry

http://pubs.rsc.org/en/content/articlelanding/2011/cc/c0cc04374e\#!divAbstract 


\section{References and Notes}

1. Beaucage, S. L.; Bergstrom, D. E.; Glick, G. D.; Jones, R. A. In Current Protocols in Nucleic Acid Chemistry, John Wiley \& Sons, Inc.: 2000.

2. (a) Beaucage, S. L. Solid-phase synthesis of siRNA oligonucleotides. Current Opinion on Drug Discovery Development 2008, 11, 203-216; (b) Schulte, M.; Luhring, N.; Keil, A.; Sanghvi, Y.S. Purification of DMT-On Oligonucleotide by Simulated Moving-Bed (SMB) Chromatography. Organic Process Research Development 2005, 9, $212-215$.

3. (a) Aitken, S.; Anderson, E., OligoPrep PVA Support for Oligonucleotide Synthesis in Columns on a Scale up to $10 \mu \mathrm{mol}$. Nucleosides, Nucleotides and Nucleic Acids 2007, 26 (8-9), 931-934; (b) Sanghvi, Y. S.; Guo, Z.; Pfundheller, H. M.; Converso, A., Improved Process for the Preparation of Nucleosidic Phosphoramidites Using a Safer and Cheaper Activator. Organic Process Research \& Development 2000, 4 (3), 175-181.

4. Pearson, W. H.; Berry, D. A.; Stoy, P.; Jung, K.-Y.; Sercel, A. D., Fluorous Affinity Purification of Oligonucleotides. The Journal of Organic Chemistry 2005, 70 (18), 71147122.

5. Deshmukh, R. R.; Miller, J. E.; De Leon, P.; Leitch, W. E.; Cole, D. L.; Sanghvi, Y. S., Process Development for Purification of Therapeutic Antisense Oligonucleotides by Anion-Exchange Chromatography. Organic Process Research Development 2000, 4, 205213.

6. (a) Fang, S.; Bergstrom, D. E., Reversible 5'-end biotinylation and affinity purification of synthetic RNA. Tetrahedron Letters 2004, 45 (43), 7987-7990; (b) Fang, 
S.; Bergstrom, D. E., Fluoride-cleavable biotinylation phosphoramidite for 5'-end-labeling and affinity purification of synthetic oligonucleotides. Nucleic Acids Research 2003, 31 (2), 708-715; (c) Fang, S.; Bergstrom, D. E., Reversible Biotinylation Phosphoramidite for 5'-End-Labeling, Phosphorylation, and Affinity Purification of Synthetic Oligonucleotides. Bioconjugate Chemistry 2002, 14 (1), 80-85

7. Beller, C.; Bannwarth, W., Noncovalent Attachment of Nucleotides by FluorousFluorous Interactions: Application to a Simple Purification Principle for Synthetic DNA Fragments. Helvetica Chimica Acta 2005, 88 (1), 171-179.

8. Sproat, B. S.; Rupp, T.; Menhardt, N.; Keane, D.; Beijer, B., Fast and simple purification of chemically modified hammerhead ribozymes using a lipophilic capture tag. Nucleic Acids Research 1999, 27 (8), 1950-1955

9. Pieken, W.; Wolter, A.; Leuck, M., Methods for the integrated synthesis and purification of oligonucleotides. U.S. Patent US20030195351 A1: 2003.

10. Fang, S.; Fueangfung, S., Scalable Synthetic Oligodeoxynucleotide Purification with Use of a Catching by Polymerization, Washing, and Releasing Approach. Organic Letters 2010, 12 (16), 3720-3723.

11. Sawadogo, M.; Dyke, M. W. V., A rapid method for the purification of deprotected oligodeoxynucleotides. Nucleic Acids Research 1991, 19 (3), 674.

12. LeProust, E. M.; Peck, B. J.; Spirin, K.; McCuen, H. B.; Moore, B.; Namsaraev, E.; Caruthers, M. H., Synthesis of high-quality libraries of long (150-mer) oligonucleotides by a novel depurination controlled process. Nucleic Acids Research 2010, 38 (8), 25222540. 
13. In our previous studies, conjugate addition of failure sequences to $N, N^{\prime}$ dimethylacrylamide was not observed. One possibility is that the reaction requires the ODN to adopt higher order structures, which can only be assumed by the full-length sequence.

14. Standing at $\mathrm{rt}$ overnight could not completely reverse the reaction.

15. The very small peaks before that of the full-length sequence, which could hardly be seen, could not be removed using this technique.

16. Zhu, Z.; Wu, C.; Liu, H.; Zou, Y.; Zhang, X.; Kang, H.; Yang, C. J.; Tan, W., An Aptamer Cross-Linked Hydrogel as a Colorimetric Platform for Visual Detection. Angewandte Chemie International Edition 2010, 49 (6), 1052-1056. 


\section{Chapter 5}

\section{Synthetic 5'-Phosphorylated Oligodeoxynucleotide Purification through \\ Catching Full-length Sequences by Polymerization*}

Yinan Yuan, ${ }^{a b}$ Suntara Fueangfung, ${ }^{a}$ Xi Lin, ${ }^{a}$ DurgaPokharel, ${ }^{a}$ and Shiyue Fang ${ }^{a}$

${ }^{a}$ Department of Chemistry, Michigan Technological University, 1400 Townsend Drive, Houghton, Michigan 49931, USA

${ }^{b}$ School of Forest Resources and Environmental Science, Michigan Technological University, 1400 Townsend Drive, Houghton, Michigan 49931, USA

Email: shifang@mtu.edu

\footnotetext{
"This material contained in this chapter was previously published in RSC Advances, 2012, 2, 2803-2808 -Reproduced by permission of The Royal Society of Chemistry. See Appendix D. Permission from publishers.
} 


\begin{abstract}
The readily scalable catching by polymerization purification technology has been further advanced to purify $5^{\prime}$-phosphorylated synthetic oligodeoxynucleotides (ODNs). The new technology utilizes a phosphoramidite that contains a fluoride-cleavable diisopropylsilyl acetal linker and a polymerizable methacrylamide group, and is capable of phosphorylation of ODN. For purification, the phosphoramidite was coupled to the $5^{\prime}$-end of full-length ODN on a synthesizer. Because failure sequences were capped in each synthetic cycle, only the full-length sequences were phosphinylated and acrylated. After cleavage and deprotection, the crude ODN was subjected to polymerization under typical acrylamide gel formation conditions. The full-length ODN was incorporated into polymer. The failure sequences and other impurities were simply removed by washing with water. Pure full-length ODN that contained a $5^{\prime}$-phosphate group was cleaved from the polymer with HF-pyridine. Reversed-phase (RP) HPLC showed that the ODN was pure, and the recovery yield was higher than that of typical preparative HPLC purification.
\end{abstract}




\subsection{Introduction}

Synthetic oligonucleotides (ONs) including ODNs and oligoribonucleotides and their unnatural analogs have found wide applications in molecular biology and other scientific areas. ${ }^{1-8}$ These ONs are synthesized on an automated synthesizer using protected nucleoside phosphoramidite monomers. ${ }^{9}$ Typically, the first monomer is anchored to a solid support via its 3 '-OH group through a cleavable linker. Phosphoramidite monomers are coupled to the $5^{\prime}-\mathrm{OH}$ in a stepwise fashion. Each synthetic cycle consists of detritylation, coupling, capping and oxidation steps, which finishes the addition of one nucleoside. Excess reagents and side products are washed away after each step while the nascent ON remains on the solid support. Normally, the coupling step is highly efficient, but generation of truncated failure sequences is unavoidable. This problem is more serious for long ONs (more than 50-mer) and large-scale ON syntheses. ${ }^{10}$ To ease purification, the failure sequences are capped with reagents such as acetic anhydride so that they do not participate in reactions in the subsequent synthetic cycles. After synthesis, the ONs are cleaved from the solid support and fully deprotected. The crude ON, which mainly contains full-length $\mathrm{ON}$, failure sequences and small organic molecule impurities resulting from deprotection, is then purified with various methods. The small organic molecules are easy to remove because they have very different physical properties from ONs. However, the separation of full-length sequence from the failure ones is difficult due to their identical physical properties. Currently used methods for $\mathrm{ON}$ purification include gel electrophoresis, RP HPLC, anion exchange HPLC, RP cartridge extraction, fluorous affinity extraction, ${ }^{10-11}$ biotin-avidin enabled affinity purification, ${ }^{12-14}$ and other

techniques. ${ }^{15}$ All these methods have limitations as we discussed in a previous paper. ${ }^{16}$ 
Briefly, gel electrophoresis can only be used to purify minute quantities of ON. HPLC methods need expensive instrument, consume large volumes of buffer, are labor intensive, and are expensive to scale up. RP cartridge extraction gives less pure ON. Fluorous affinity extraction and biotin-avidin enabled affinity purification require solid phase extraction materials, which are normally not reusable. To solve the ON purification problem, we recently developed two methods that utilized the concept of catching by polymerization for ODN purification. ${ }^{16-17}$ In one method, the failure sequences were capped with an acrylated phosphoramidite in each synthetic cycle. After synthesis, purification was achieved by simply polymerizing the failure sequences. ${ }^{17}$ In the second method, the full-length sequences were coupled to an acrylated phosphoramidite. After synthesis, purification was achieved by polymerization of full-length sequences, washing away the impurities and cleaving pure ODN from polymer. ${ }^{16}$ In this chapter, we report our results on using the catching by polymerization technology to purify synthetic ODNs that contain a $5^{\prime}$ phosphate group.

\subsection{Results and Discussion}

In some biological applications such as ligation of synthetic ODNs for gene synthesis, ${ }^{18}$ cloning, ${ }^{19}$ mutagenesis, ${ }^{20}$ and ligation chain reaction, ${ }^{21}$ a phosphate group at the $5^{\prime}$-end of ODN is required. This group can be installed via enzyme-catalyzed phosphorylation. ${ }^{19}$ A more convenient approach is to attach the group on an automated DNA synthesizer. Several reagents have been commercialized for the purpose. ${ }^{22-23}$ One example is the phosphoramidite of 4,4'-dimethoxytrityl (DMTr)-protected bis(hydroxymethyl)malonate. ${ }^{23}$ The DMTr group in this reagent serves as a hydrophobic 
handle for RP HPLC purification after synthesis. At the end of purification, the group is removed and the phosphate group at the $5^{\prime}$-end of ODN is released under basic conditions. In this paper, we combine this chemical phosphorylation technology with our newly developed catching by polymerization ODN purification technology to develop a method for purification of $5^{\prime}$-phosphorylated ODN. The reagent we designed for this ODN phosphorylation and purification

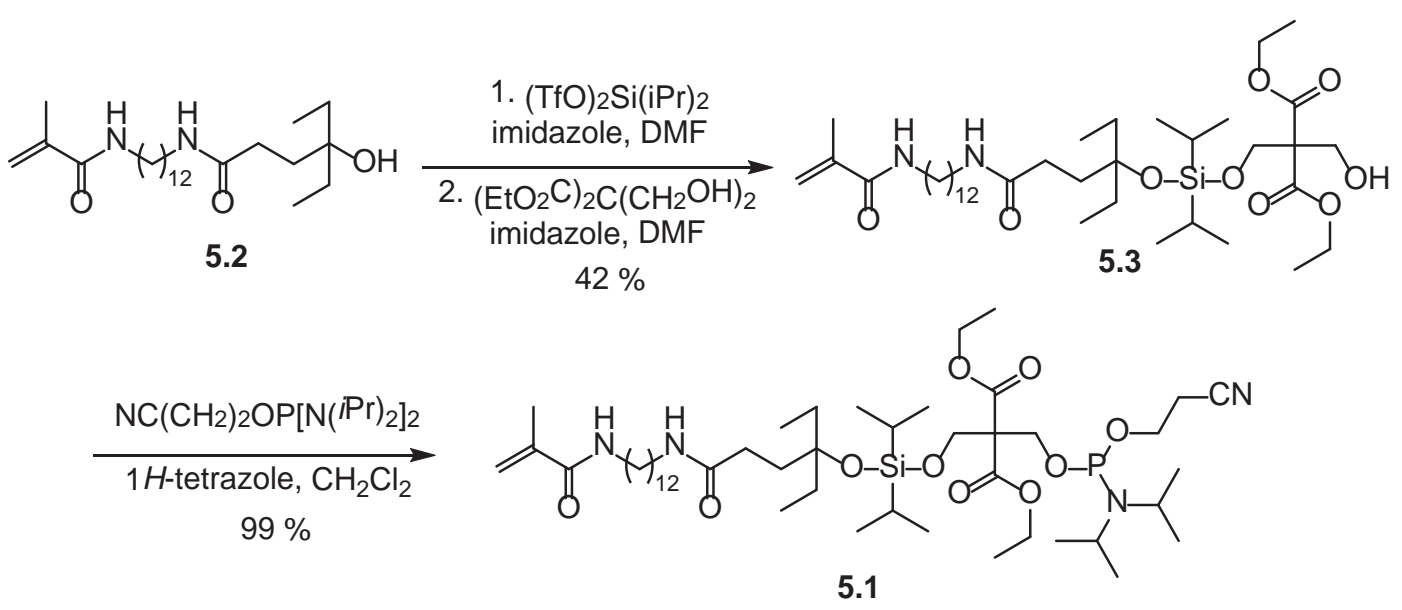

Scheme 5.1. Synthesis of acylation and phosphinylation phosphoramidite $\mathbf{5 . 1}$

application is phosphoramidite 5.1. Its synthesis is shown in Scheme 5.1. The easily accessible and known methacryloyl tertiary alcohol $\mathbf{5 . 2}^{16}$ was coupled to diethyl bis(hydroxymethyl) malonate with dichlorodiisopropylsilane to give 5.3. The alcohol $\mathbf{5 . 3}$ was then phosphinylated to give the target phosphoramidite 5.1. To demonstrate the usefulness of the phosphorylation and purification technique, the acrylated ODN 5.4 (see Scheme 5.2) was synthesized on a DNA synthesizer. Typical UltraMild ODN synthesis conditions, which use the more base-labile phenoxyacetyl protecting groups and allow deprotection with concentrated ammonium hydroxide at room temperature (see 
experimental section for details), were used. At the end of the synthesis, the phosphoramidite 5.1 was coupled to the $5^{\prime}$-end of the ODN for $5 \mathrm{~min}$. Oxidation and capping in this cycle were carried out under normal conditions. The cleavage and deprotection were carried out on the synthesizer. The crude ODN contained the full-length sequence 5.4, and impurities, which included the failure sequences $\mathbf{5 . 5}$ and small molecules from protecting groups. Because the failure sequences were capped with phenoxyacetic anhydride $\left(\mathrm{Pac}_{2} \mathrm{O}\right)$ in each synthetic cycle, they did not react with the phosphoramidite 5.1, and did not contain a methacrylamide group. Only the full-length sequences were acrylated. The crude ODN was analyzed with RP HPLC. As shown in Figure 5.1, the failure sequences $\mathbf{5 . 5}$ appeared at around $19 \mathrm{~min}$. Due to the relatively more hydrophobic tag at the $5^{\prime}$-end of 5.4, the full-length ODN had an unusually long retention time of 62 min. The small peaks appearing at 31 and 59 min may be attributed to small molecules from protecting groups.

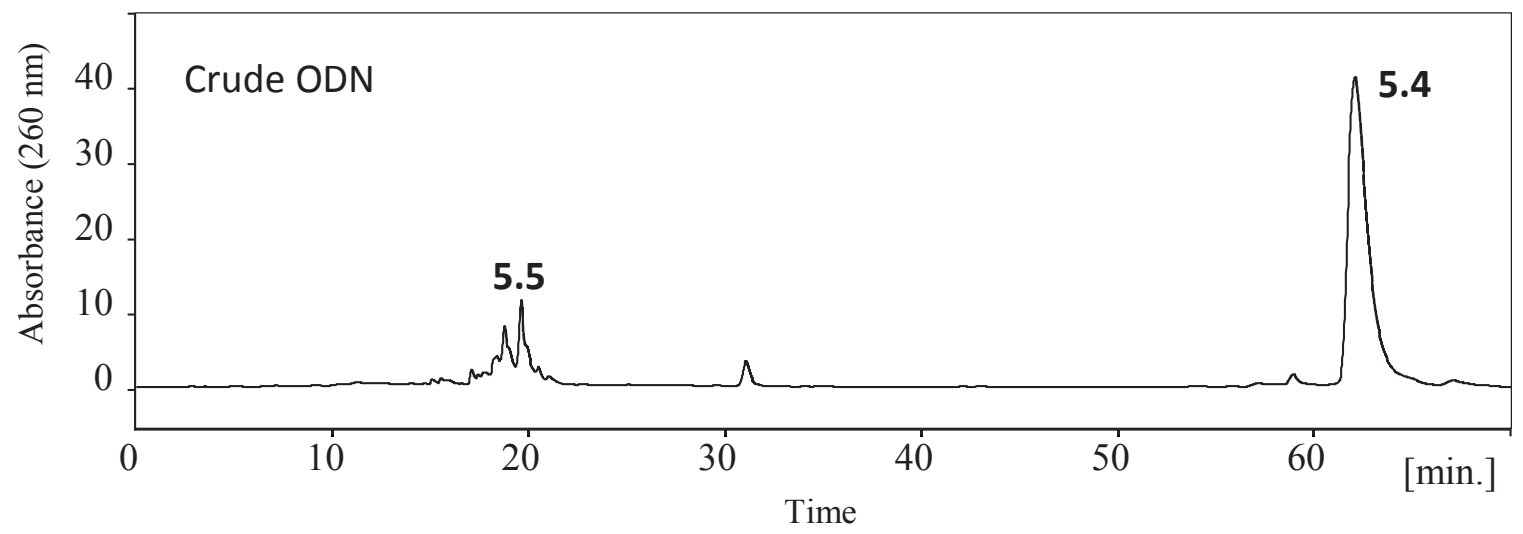

Figure 5.1. RP HPLC profile of crude ODN 
The crude ODN was subjected to polymerization under typical polyacrylamide gel formation conditions (Scheme 5.2). Specifically, $N, N$-dimethylacrylamide was used as the monomer, a small amount of $N, N^{\prime}$-methylenebis(acrylamide) was used as the cross-linker, water was used as the solvent, and the polymerization was conveniently initiated with ammonium persulfate and $N, N, N^{\prime}, N^{\prime}$-tetramethylethylenediamine (TMEDA). The reaction could tolerate air, but was conducted under nitrogen to minimize radical termination reactions by oxygen. The reaction was completed within $30 \mathrm{~min}$ at room temperature. To ensure that all monomers and cross-linkers were incorporated into the polymer, the reaction mixture was allowed to stand under nitrogen at room temperature for another $30 \mathrm{~min}$. The acrylated full-length sequence $\mathbf{5 . 4}$ was incorporated into the polymer to give the ODNpolymer conjugate 5.6 (Scheme 5.2). The failure sequences $\mathbf{5 . 5}$ and the impurities resulting from protecting groups remained in the polymer matrix, which were conveniently removed by extraction with water. The extracts were analyzed with RP HPLC to give a trace in Figure 5.2. Compared with the trace in Figure 5.1, the trace in Figure 4.2 did not have a peak at $62 \mathrm{~min}$, which indicated that the full-length sequence was incorporated into polymer 5.6 efficiently.

The ODN-polymer conjugate 5.6 was dried under vacuum. The intermediate ODN 5.7 was cleaved from polymer with HF-pyridine complex in DMF. Excess HF was quenched with $\mathrm{Me}_{3} \mathrm{SiOMe}$. The supernatant, which contained the ODN 5.7, excess $\mathrm{Me}_{3} \mathrm{SiOMe}, \mathrm{Me}_{3} \mathrm{SiF},(\mathrm{iPr})_{2} \mathrm{SiF}_{2}, \mathrm{MeOH}$, and the solvent $\mathrm{DMF}$, was removed. The polymer was washed with water. The supernatant and the washes were combined and evaporated to dryness leaving only the ODN 5.7. To remove the tag on the $5^{\prime}$-phosphate group, to $\mathbf{5 . 7}$ was added concentrated ammonium hydroxide solution and heated to $80{ }^{\circ} \mathrm{C}$ for $30 \mathrm{~min}$. 
Pure 5'-phosphorylated ODN 5.8 was obtained conveniently by direct $n$-BuOH precipitation from the solution. As shown in Figure 5.3, only one peak with a retention time of 19 min was observed. The recovery yield of the purification process was estimated to be $55 \%$ by comparing the area of the peak at 19 min in Figure 5.3 with the area of the peak at $62 \mathrm{~min}$ in Figure 5.1. The identity of the ODN was confirmed by MALDI-TOF MS analysis. 


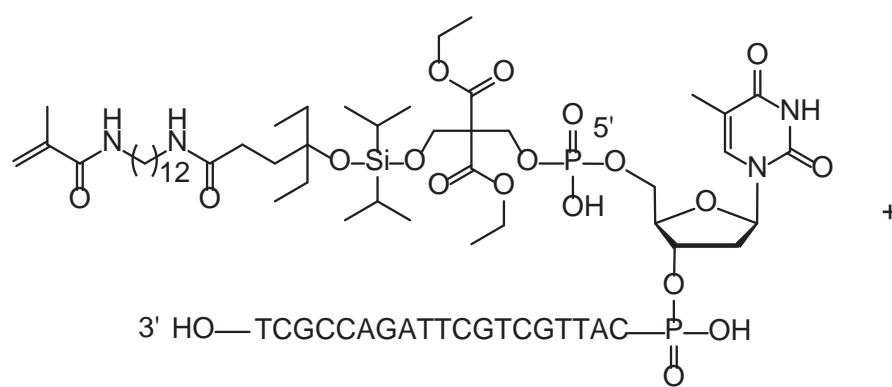

Full-length sequence 5.4

thylacrylamide $N, N$ '-methylenebis(acrylamide) $\left(\mathrm{NH}_{4}\right)_{2} \mathrm{~S}_{2} \mathrm{O}_{8}$, TMEDA, $\mathrm{H}_{2} \mathrm{O}$, rt, $1 \mathrm{~h}$

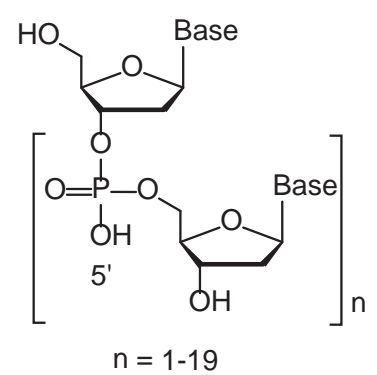

Failure sequences $\mathbf{5 . 5}$

2. Wash with water to remove $\mathbf{5 . 5}$ and other impurities

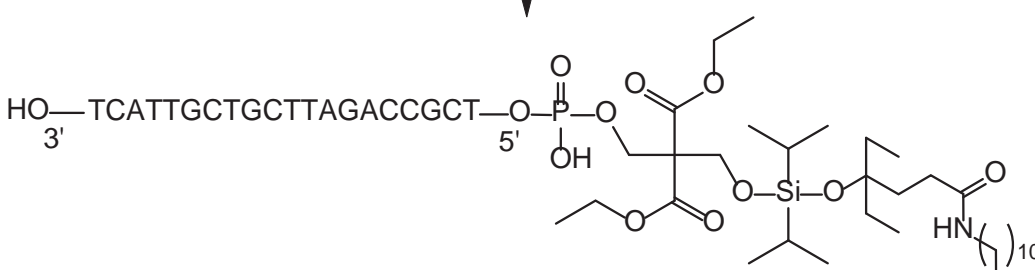

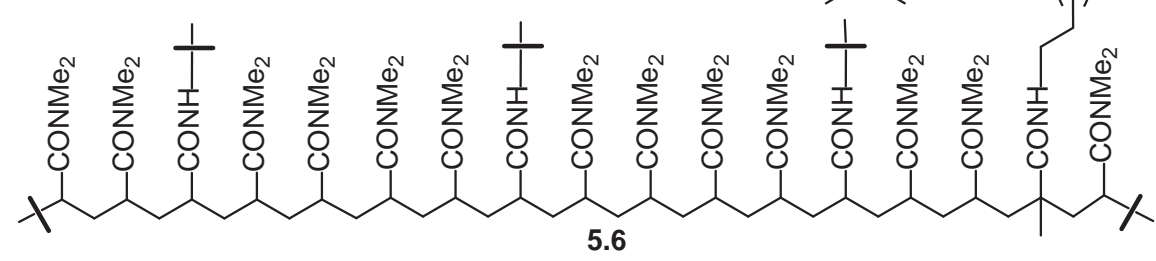

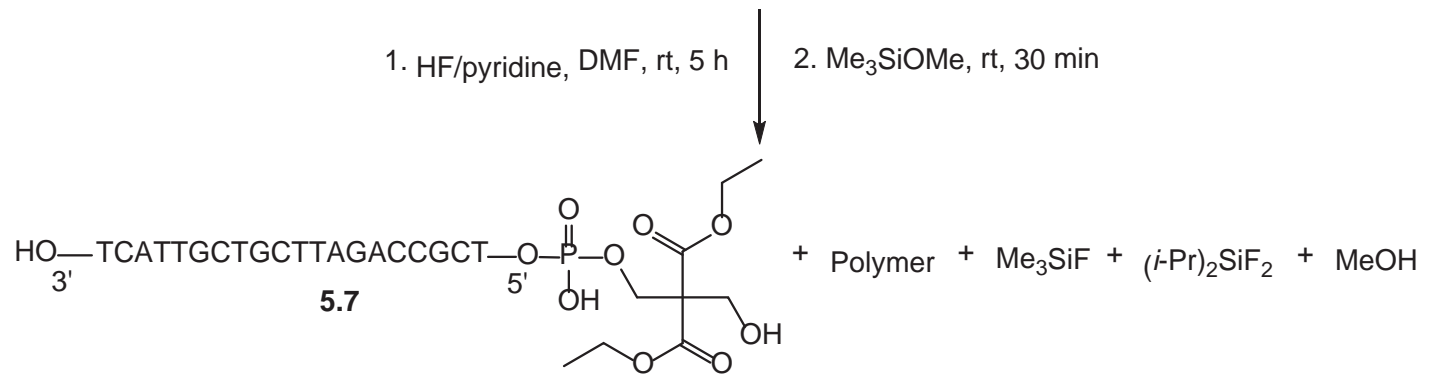

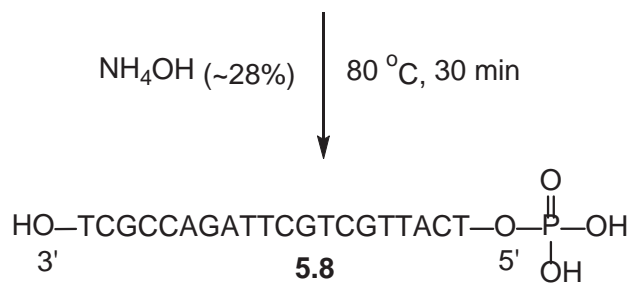

Scheme 5.2. Purification of 5'-phosphorylated ODN through catching full-length sequence by polymerization 


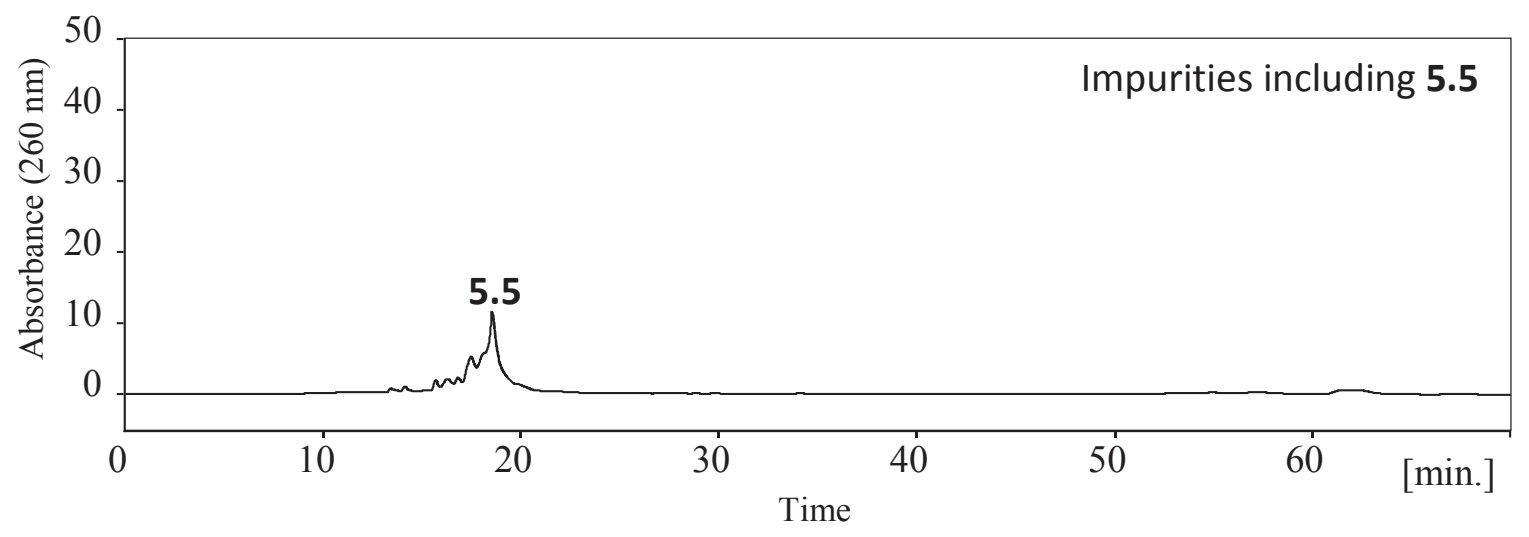

Figure 5.2. RP HPLC profile of impurities including failure sequences $\mathbf{5 . 5}$

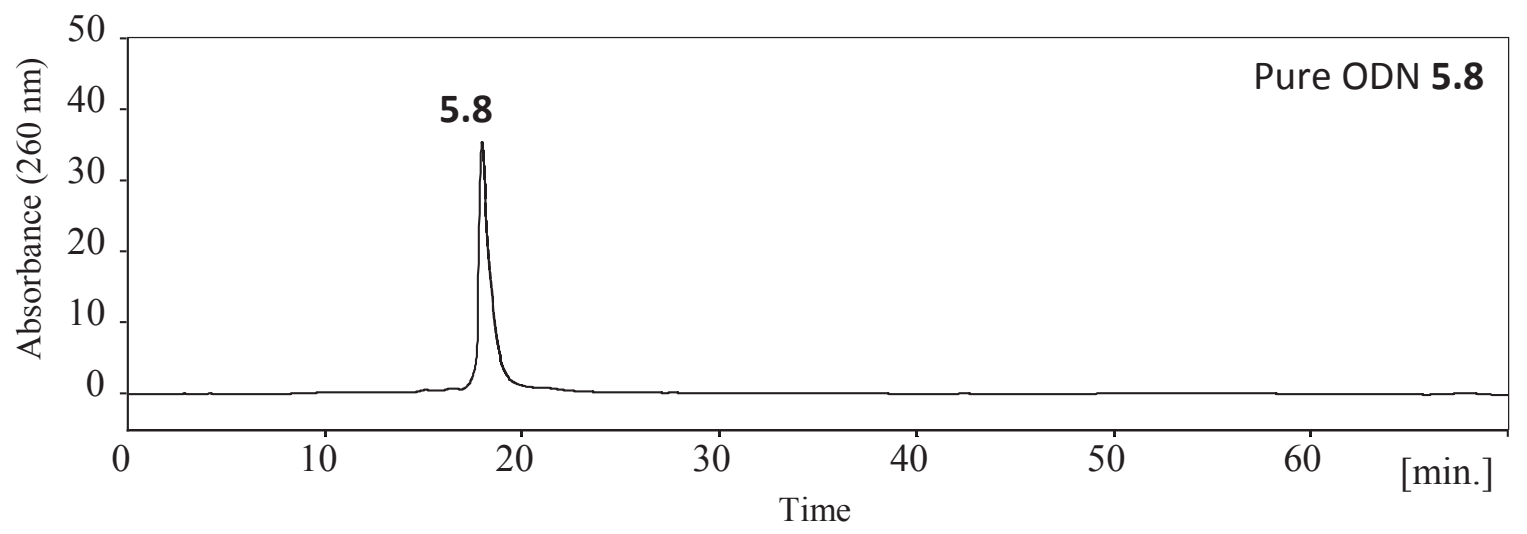

Figure 5.3. RP HPLC profile of purified ODN $\mathbf{5 . 8}$ 
To further demonstrate the usefulness of this chemical phosphorylation and purification technology, the 61-mer ODN 5.9 (Figure 5.4), which is a portion of HIV protease gene, was synthesized and phosphinylated with phosphoramidite 5.1. To increase the yield of the synthesis, controlled pore glass (CPG) with a pore size of $2000 \AA$ was used. Before starting the automated synthesis, the CPG was manually capped with $\mathrm{Pac}_{2} \mathrm{O}$ for 20 min on the synthesizer. In addition, in the synthetic cycle, a 25 -second waiting step was added after each delivery of coupling reagents (phosphoramidite and $1 \mathrm{H}$-tetrazole) to the synthesis column. The capping step was performed two times. Following each capping step, a 50-second waiting step was added. Except for these modifications, the same synthesis procedure for ODN 5.4 was followed. The crude 5.9 was analyzed with RPHPLC (trace a, Figure 5.5), and purified with the catching by polymerization procedure as described for purification of 5.4. The purified 61-mer ODN $\mathbf{5 . 1 0}$ was analyzed with RPHPLC to give trace $\mathrm{b}$ (Figure 5.5). The recovering yield was estimated to be $65 \%$ by comparing the area of the peak at $20 \mathrm{~min}$ in the trace $\mathrm{b}$ with the peak area at $54 \mathrm{~min}$ in trace a (Figure 5.5). 


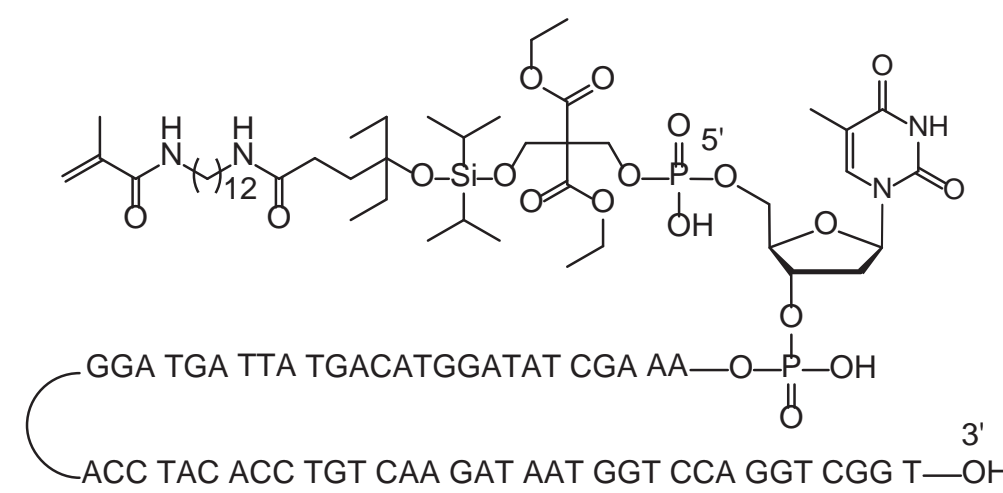

5.9

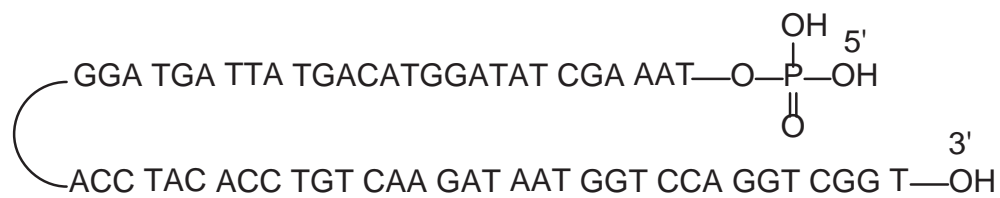

5.10

Figure 5.4. Sequence of ODN 5.9, and $\mathbf{5 . 1 0}$ 

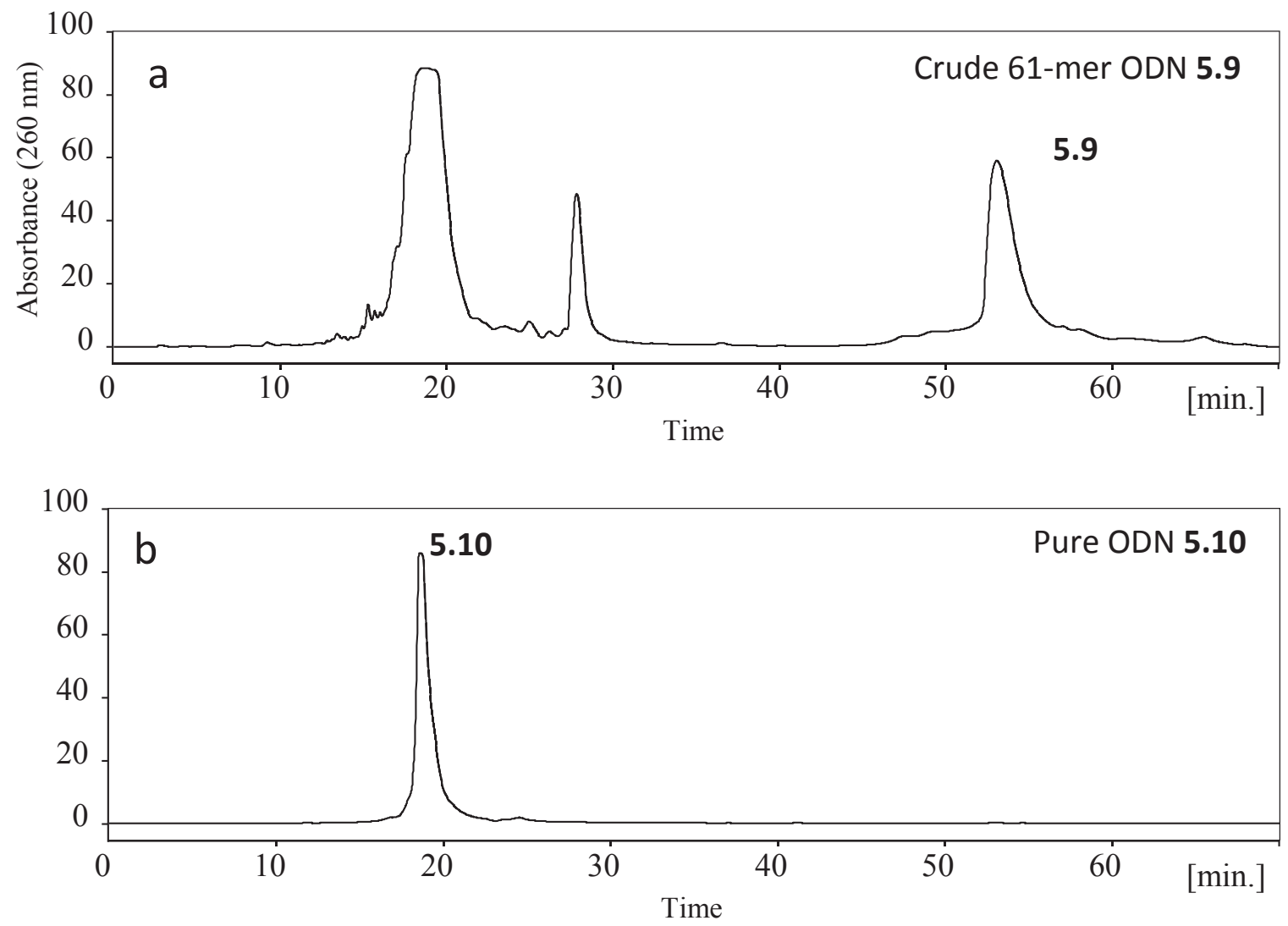

Figure 5.5. RP HPLC profiles: (a) crude 61-mer ODN 5.9; (b) purified 61-mer ODN 5.10

To avoid an extra heating step during deprotection of ODN, initially we used the UltraMild conditions for the synthesis of ODNs 5.4 and 5.9. However, in the more widely used base protecting strategy in DNA synthesis, dA is protected with a benzoyl group and $\mathrm{dG}$ is protected with an isobutyryl group. These protecting groups require heating in concentrated ammonium hydroxide for $8 \mathrm{~h}$ to remove. To test if our chemical phosphorylation and catching by polymerization techniques are compatible with these relatively harsher conditions, the 25-mer ODN 5.11 (Figure 5.6) was synthesized using this normal protecting strategy. Specifically, phosphoramidite monomers were Bz-dA, $i$-Bu$\mathrm{dG}, \mathrm{Ac}-\mathrm{dC}$ and $\mathrm{dT}$. The capping reagent was acetic anhydride. Except for these 
modifications, all other reagents and conditions including the synthesis cycle are the same as those for the synthesis of 5.4. ODN 5.11 was cleaved from CPG with concentrated ammonium hydroxide at room temperature. The solution was then heated to $55^{\circ} \mathrm{C}$ for $8 \mathrm{~h}$ to remove protecting groups. The crude $\mathbf{5 . 1 1}$ was analyzed with HPLC (trace a, Figure 5.7) and purified with the catching by polymerization procedure as described for purification of 5.4. The purified 25-mer ODN 5.12 (Figure 5.6) was analyzed with HPLC to give trace $b$ (Figure 5.7). The recovering yield was estimated to be $79 \%$ by comparing the area of the peak at $18 \mathrm{~min}$ in trace $\mathrm{b}$ (Figure 5.7) with that of the peak at $58 \mathrm{~min}$ in the trace a (Figure $5.7)$

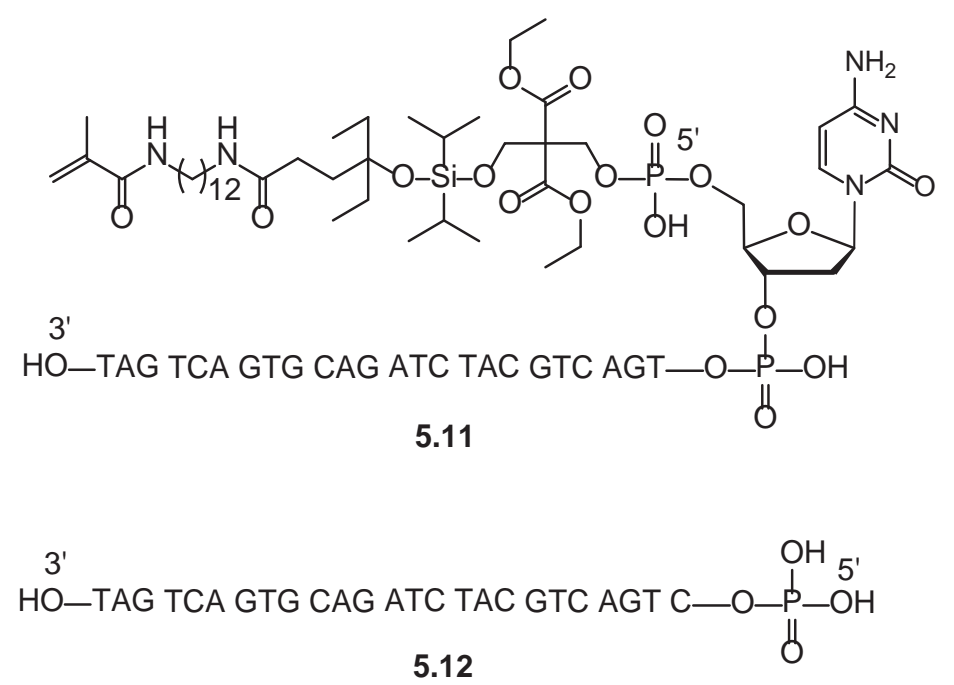

Figure 5.6. Sequence of ODN 5.11, and 5.12 

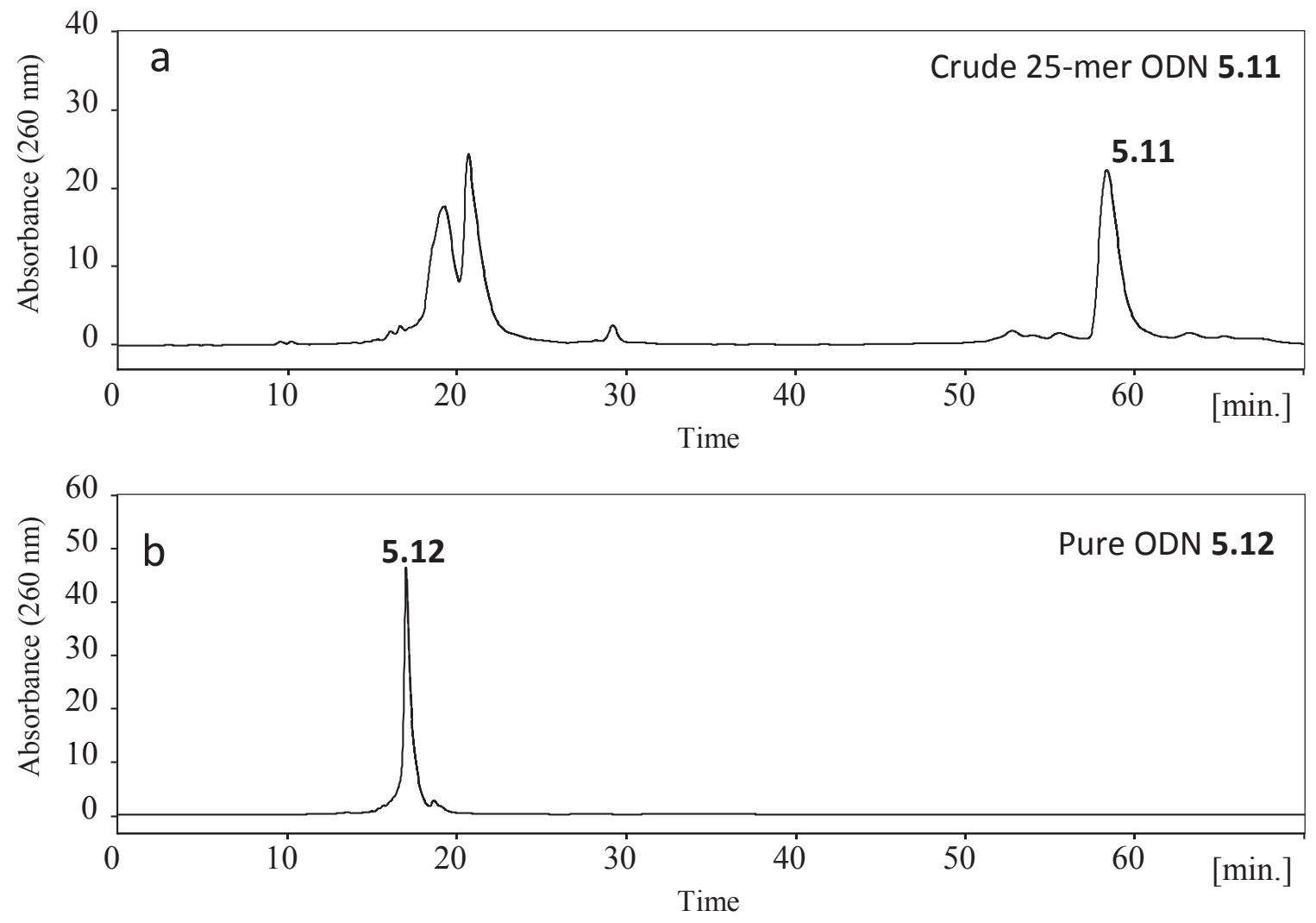

Figure 5.7. RP HPLC profiles: (a) crude 25-mer ODN 5.11; (b) purified 25-mer ODN 5.12

This new ODN phosphorylation and purification technology provides a convenient method to access 5'-phosphorylated ODNs. We have found that the acrylation phosphoramidite $\mathbf{5 . 1}$ is stable for at least one month when stored at $-20{ }^{\circ} \mathrm{C}$ under nitrogen in the dark.

In the future, when this compound or its revised version is commercialized, highly pure 5'-phosphorylated ODNs can be obtained using the following procedure: (1) Coupling the acrylation phosphoramidite to the ODN at the end of synthesis. (2) Perform cleavage 
and deprotection as usual. (3) Subject the crude ODN to polymerization. (4) Wash the polymer with water. (5) Cleave ODN from the polymer. (6) Treat ODN with concentrated ammonium hydroxide shortly and precipitate ODN with $n$-BuOH. Several advantages of this technology are remarkable. When compared with HPLC, this technology requires minimum volume of solvent; the cost of the HPLC instrument and its maintenance fee can also be saved. When compared with fluorous affinity purification and biotin-streptavidin enabled affinity purification methodologies, which are also efficient and convenient, this new technology does not require any solid phase extraction material. The chemicals for the polymerization reaction are all commercially available, inexpensive, and can be stored for extended periods of time under suitable conditions. These materials are only needed in small quantities. The most significant advantage of the new technology is that it can be readily scaled up. Because there is no need of any type of chromatography, and purification is achieved by simple manipulations such as shaking and extraction, large quantities of ODN can be purified in each batch. This can be easily envisioned by the fact that there is virtually no cost difference between purification of $1 \mathrm{mg}$ of ODN and purification of $1 \mathrm{~g}$ of ODN. If any other methods are used, the difference could be enormous. Due to these advantages, we expect that the technology presented in this paper will be widely used in industry and academia for the production of pure $5^{\prime}$-phosphorylated ODNs. 


\subsection{Conclusions}

In conclusion, by combining a commercialized chemical phosphorylation technique and our newly developed ODN purification methodology, we have developed a new technology for the purification of synthetic $5^{\prime}$-phosphorylated ODN. The technology is simple, convenient, inexpensive, and highly efficient. It may be readily scaled up and affords pure ODN at both small and large scales. We believe that it will provide a more affordable way for scientists to obtain 5'-phosphorylated ODNs for applications in chemistry, biology and medicine.

\subsection{Experimental}

\section{General}

All reactions were performed in oven-dried glassware under a nitrogen atmosphere using standard Schlenk techniques. Reagents and solvents available from commercial sources were used as received unless otherwise noted. $\mathrm{CH}_{2} \mathrm{Cl}_{2}$ was distilled over $\mathrm{CaH}_{2}$. Thin layer chromatography (TLC) was performed using Sigma-Aldrich TLC plates, silica gel 60F-254 over glass support, $0.25 \mathrm{~mm}$ thickness. Flash column chromatography was performed using Selecto Scientific silica gel, particle size $32-63 \mathrm{~mm} .{ }^{1} \mathrm{H},{ }^{13} \mathrm{C}$ and ${ }^{31} \mathrm{P} \mathrm{NMR}$ spectra were measured on a Varian UNITY INOVA spectrometer at 400, 100 and 162 $\mathrm{MHz}$, respectively; chemical shifts $(\delta)$ were reported in reference to solvent peaks (residue $\mathrm{CHCl}_{3}$ at $\delta 7.24 \mathrm{ppm}$ for ${ }^{1} \mathrm{H}$ and $\mathrm{CDCl}_{3}$ at 77.00 ppm for ${ }^{13} \mathrm{C}$ ) and $\mathrm{H}_{3} \mathrm{PO}_{4}($ at $\delta 0.00 \mathrm{ppm}$ for $\left.{ }^{31} \mathrm{P}\right)$. High resolution mass spectra were obtained on a Finnigan Mat 95XL spectrometer. MALDI-TOF mass spectrum was obtained on a Shimadzu Biotech Axima CFRplus spectrometer. ODNs were synthesized on an ABI 394 solid phase synthesizer. HPLC was 
performed on a JASCO LC-2000Plus System, Pump PU- 2089Plus Quaternary Gradient Pump, Detector UV-2075Plus. C-18 RP analytical column (5 mm diameter, $100 \AA$, $250 \times$ $3.20 \mathrm{~mm}$ ) was used. Solvent A: $0.1 \mathrm{M}$ triethylammonium acetate, 5\% acetonitrile; solvent B: $90 \%$ acetonitrile. All profiles were generated by detection of absorbance of DNA at 260 $\mathrm{nm}$ using the linear gradient solvent system: solvent B (0-45\%) in solvent A over $60 \mathrm{~min}$ followed by solvent B (45\%-100\%) in solvent A over $20 \mathrm{~min}$ at a flow rate of $0.5 \mathrm{~mL} \mathrm{~min}{ }^{-}$

1. THF/pyridine/Pac $2 \mathrm{O}, \mathrm{THF} /$ pyridine/Ac $2 \mathrm{O}$, succinic ester linked DMTr-dTlcaa- CPG (pore sizes $1000 \AA$ and $2000 \AA$; lcaa = long chain alkylamino), 5'-DMTr 2-cyanoethyl phosphoramidites acetyl-dC, Pac-dA (Pac = phenoxyacetyl), Bz-dA, 4-isopropyl-Pac-dG, $i$-Bu-dG and $\mathrm{dT}$, and other commonly used solid phase DNA synthesis reagents were purchased from Glen Research, Inc.

\section{Synthesis of compound 5.3}

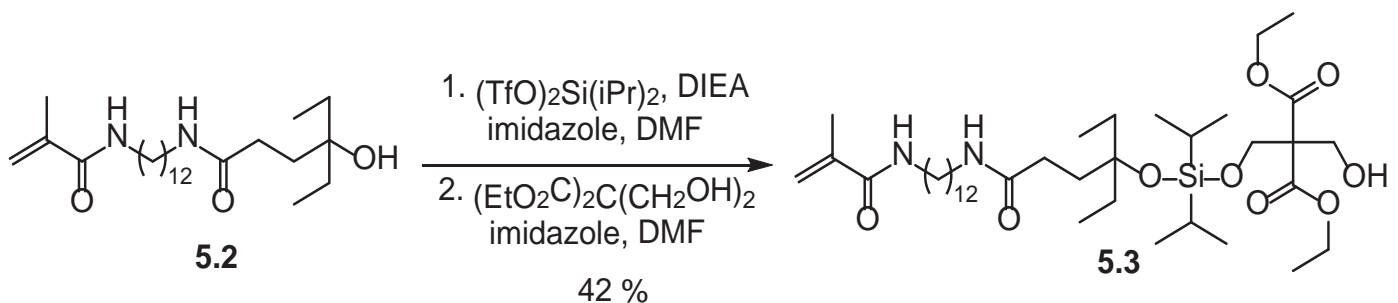

A round-bottomed flask containing compound $\mathbf{5 . 2 ^ { 1 6 }}(200 \mathrm{mg}, 0.49 \mathrm{mmol}, 1.0$ eq.) and a magnetic stirring bar was evacuated and then refilled with nitrogen. The evacuation and nitrogen filling cycle was repeated for two more times. Dry DMF $(2 \mathrm{~mL})$ and diisopropylethylamine ( $254 \mathrm{~mL}, 1.46 \mathrm{mmol}, 3.0$ eq.) were added via syringes. The mixture was cooled to $0 \mathrm{uC}$. Diisopropylsilyl bis(trifluoromethane sulfonate) (144 mL, 0.487 mmol, 1.0 eq. $)$ in dry DMF $(1 \mathrm{~mL})$ was added via a syringe in one portion at $0{ }^{\circ} \mathrm{C}$. The 
solution was stirred at $0{ }^{\circ} \mathrm{C}$ for $1 \mathrm{~h}$ and $\mathrm{rt}$ for $2 \mathrm{~h}$. Imidazole $(50 \mathrm{mg}, 0.73 \mathrm{mmol}, 1.5 \mathrm{eq}$.) in dry DMF ( $1 \mathrm{~mL})$ was added via a syringe. The solution was stirred for $1 \mathrm{~h}$, and then added to a flask containing diethyl bis(hydroxymethyl)malonate (107 $\mathrm{mg}, 0.487 \mathrm{mmol}, 1.0 \mathrm{eq}$.$) ,$ imidazole (33 mg, $0.487 \mathrm{mmol}, 1.0$ eq.) and $\operatorname{DMF}(2 \mathrm{~mL})$ at $0{ }^{\circ} \mathrm{C}$ via a cannula slowly. The reaction mixture was stirred at $0{ }^{\circ} \mathrm{C}$ for $4 \mathrm{~h}$, and then quenched with $5 \% \mathrm{NaHCO}_{3}\left(0{ }^{\circ} \mathrm{C}, 50\right.$ $\mathrm{mL})$. EtOAc $\left(0{ }^{\circ} \mathrm{C}, 30 \mathrm{~mL}\right)$ was added, and the phases were separated. The aqueous phase was extracted with EtOAc $\left(0^{\circ} \mathrm{C}, 30 \mathrm{~mL} \times 3\right)$. The combined organic phase was dried over anhydrous $\mathrm{Na}_{2} \mathrm{SO}_{4}$ and filtered. The filtrate was evaporated under reduced pressure to give a yellow oil. Purification with flash column chromatography $\left(\mathrm{SiO}_{2}\right.$, hexanes/EtOAc, $3: 1$ to $1: 1)$ gave 5.3 as a pale yellow oil $(151 \mathrm{mg}, 42 \%): \mathrm{R}_{f} 0.60\left(\mathrm{SiO}_{2}\right.$, hexanes/EtOAc $=1$ : 2); ${ }^{1} \mathrm{H}$ NMR (400 MHz, $\mathrm{CDCl}_{3}$ ) $\delta 6.07$ (br s, 1H), 5.94 (br s, 1H), 5.614-5.609 (m, 1H), 5.25-5.24 (m, 1H), 4.23 (s, 2H), 4.21-4.08 (m, 6H), 3.86 (br s, 1H), 3.26-3.21 (m, 2H), 3.18-3.13 (m, 2H), 2.26-2.14 (m, 2H), 1.91-1.90 (m, 3H), 1.83-1.77 (m, 2H), 1.53- 1.37 (m, 8H), 1.25-1.19 (m, 22H), 0.99-0.95 (m, 14H), 0.83-0.76 (m, 6H); ${ }^{13} \mathrm{C}$ NMR (100 $\left.\mathrm{MHz}, \mathrm{CDCl}_{3}\right) \delta 173.8,169.3,168.7,140.4,119.3,78.8,61.8,61.6,61.5,61.4,39.9,39.8$, 34.7, 31.6, 31.3, 31.1, 30.9, 29.7, 29.6, 29.4, 27.1, 18.9, 18.1, 17.9, 14.4, 14.2, 13.7, 8.5; HRMS (ESI, $[\mathrm{M}+\mathrm{Na}]^{+}$) calculated for $\mathrm{C}_{39} \mathrm{H}_{74} \mathrm{~N}_{2} \mathrm{NaO}_{9} \mathrm{Si}$ 765.5061, found 765.5069. 


\section{Synthesis of phosphoramidite 5.1}

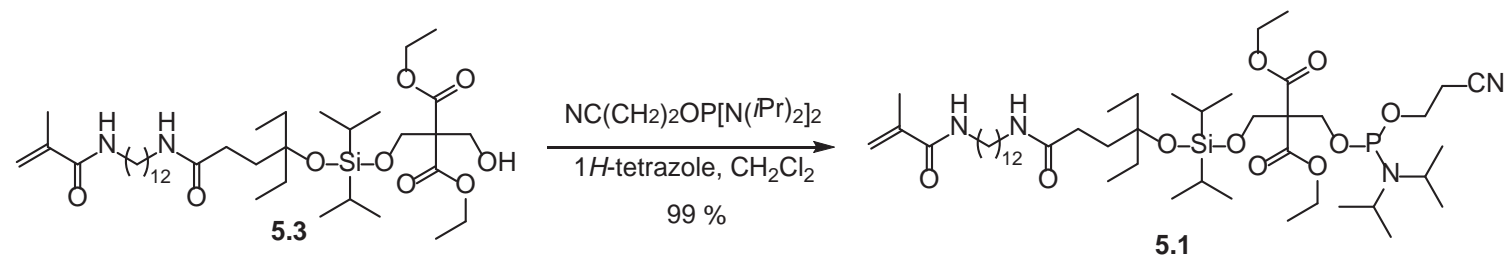

A round-bottomed flask containing $5.3(119 \mathrm{mg}, 0.16 \mathrm{mmol}, 1.0$ eq.) and a magnetic stirring bar was evacuated and then refilled with nitrogen. The evacuation and nitrogen-filling cycle was repeated for two more times. Dry $\mathrm{CH}_{2} \mathrm{Cl}_{2}(5 \mathrm{~mL})$ and 2cyanoethyl- $N, N, N^{\prime}, N^{\prime}$-tetraisopropylphosphoramidite $(60 \mathrm{~mL}, 0.176 \mathrm{mmol}, 1.1$ eq.) were then added via syringes. A

$1 H$-tetrazole solution in $\mathrm{CH}_{3} \mathrm{CN}(0.45 \mathrm{M}, 391 \mathrm{~mL}, 0.176 \mathrm{mmol}, 1.1$ eq.) was added via a syringe in one portion. After stirring at $\mathrm{rt}$ for $2 \mathrm{~h}$, the reaction mixture was concentrated to dryness under reduced pressure. The residue was purified with flash column chromatography $\left(\mathrm{SiO}_{2}\right.$, hexanes/EtOAc/Et $\left.3 \mathrm{~N}=3: 1: 1\right)$ giving 5.1 as a colorless oil (150 mg, 99\%): $\mathrm{R}_{f} 0.40\left(\mathrm{SiO}_{2}\right.$, hexanes/EtOAc/ $\left.\mathrm{Et}_{3} \mathrm{~N}=3: 1: 1\right) ;{ }^{1} \mathrm{H} \mathrm{NMR}\left(400 \mathrm{MHz}, \mathrm{CDCl}_{3}\right) \delta$ 6.00 (br s, 1H), 5.85 (br s, 1H), 5.64-5.60 (m, 1H), 5.263-5.257 (m, 1H), 4.23-4.10 (m, $8 \mathrm{H}), 3.80-3.71(\mathrm{~m}, 2 \mathrm{H}), 3.56-3.47(\mathrm{~m}, 2 \mathrm{H}), 3.28-3.23(\mathrm{~m}, 2 \mathrm{H}), 3.20-3.15(\mathrm{~m}, 2 \mathrm{H}), 2.58-$ $2.55(\mathrm{~m}, 2 \mathrm{H}), 2.27-2.15(\mathrm{~m}, 2 \mathrm{H}), 1.921-1.920(\mathrm{~m}, 3 \mathrm{H}), 1.85-1.74(\mathrm{~m}, 2 \mathrm{H}), 1.56-1.38(\mathrm{~m}$ $8 \mathrm{H}), 1.24-1.18(\mathrm{~m}, 24 \mathrm{H}), 1.14-1.11(\mathrm{~m}, 10 \mathrm{H}), 1.00-0.98(\mathrm{~m}, 14 \mathrm{H}), 0.82(\mathrm{t}, 6 \mathrm{H}, \mathrm{J}=7.6$ $\mathrm{Hz}) ;{ }^{13} \mathrm{C} \mathrm{NMR}\left(100 \mathrm{MHz}, \mathrm{CDCl}_{3}\right) \delta 173.6,168.6,140.5,119.2,117.7,79.1,61.7,61.5$, $61.1,61.0,58.7,58.5,43.4,43.3,39.9,39.7,35.6,34.2,31.9,31.1,29.8,29.71,29.67$, $29.59,29.50,29.46,27.1,24.8,24.74,24.69,24.66,20.55,20.49,18.9,18.1,17.9,14.4$, 14.1, 13.6, 8.53; ${ }^{31} \mathrm{P}$ NMR (162 MHz, $\left.\mathrm{CDCl}_{3}\right) \delta 149.9$. 


\section{ODN 5.4 synthesis, cleavage and deprotection}

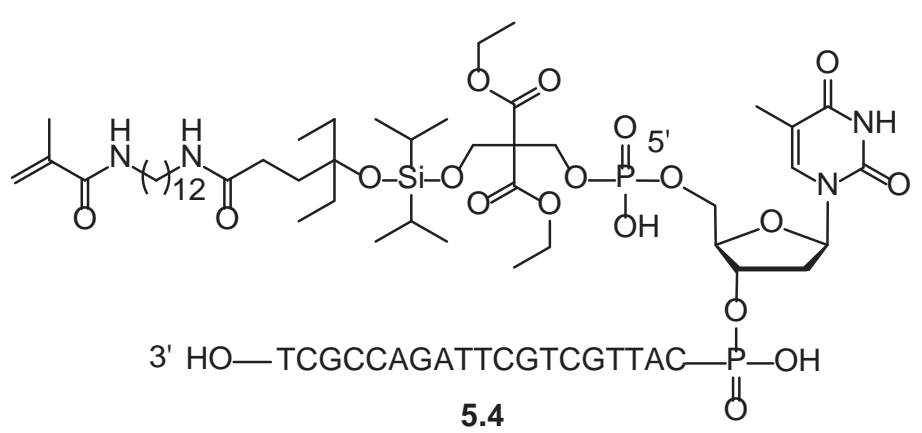

The 20-mer 5'-acrylated and phosphorylated ODN 5.4 was synthesized on an ABI DNA/RNA synthesizer at $1 \mathrm{mmol}$ scale. The solid support was lcaa-CPG with a pore size of $1000 \AA$. The ODN was anchored to the support through a succinic ester linkage. The following 5'-DMTr-protected 2-cyanoethyl phosphoramidite monomers were used for the synthesis: Pac-dA, 4-isopropyl-Pac-dG, Ac-dC and dT. THF/pyridine/Pac ${ }_{2} \mathrm{O}$ was used as the capping reagent. The manufacturer recommended synthetic cycle was followed except that the phosphoramidite $\mathbf{5 . 1}$ was coupled for $5 \mathrm{~min}$. After synthesis, the ODN was cleaved from $\mathrm{CPG}$ with concentrated $\mathrm{NH}_{4} \mathrm{OH}$ at $\mathrm{rt}$ and allowed to stand under these conditions for $8 \mathrm{~h}$. The solution of crude ODN was divided into 10 equal portions, and evaporated to dryness in 10 Eppendorf tubes in a vacuum SpeedVac concentrator. One portion was dissolved in $150 \mathrm{~mL}$ water, of which $20 \mathrm{~mL}$ was injected into HPLC to generate trace in Figure 5.1. 


\section{Polymerization of full-length ODN 5.4-synthesis of ODN-polyacrylamide conjugate}

\section{6}

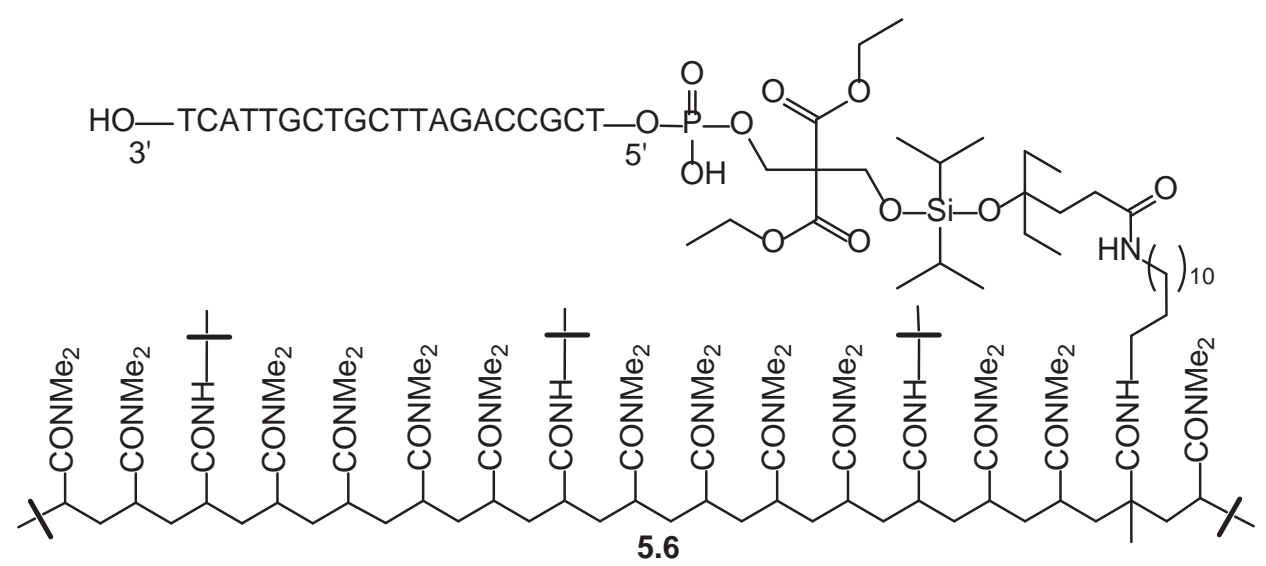

The remaining $130 \mathrm{~mL}$ solution of the crude ODN 5.4 was transferred into a 2necked round-bottomed flask. The Eppendorf tube was washed with water $(40 \mathrm{~mL} \times 3)$ and the washes were added to the flask. A polymerization solution $[250 \mathrm{~mL} ; N, N$ dimethylacrylamide (1.69 M) and $N, N^{\prime}$-methylenebis(acrylamide) $(16.9 \mathrm{mM})$ in water; the solution can be pre-prepared and stored at $-20^{\circ} \mathrm{C}$ in the dark for at least 1 month] was added via a pipette. The mixture was gently stirred under a nitrogen flow for 2 min. The solution of $\left(\mathrm{NH}_{4}\right)_{2} \mathrm{~S}_{2} \mathrm{O}_{4}(10 \%, 5 \mathrm{~mL})$ was then added via a pipette, which was followed by $N, N, N^{\prime}, N^{\prime}$-tetramethylethylenediamine (TMEDA, $5 \mathrm{~mL}$ ). The mixture was stirred gently under nitrogen at rt for $30 \mathrm{~min}$. The ODN-polyacrylamide conjugate $\mathbf{5 . 6}$ was formed. The failure sequences $\mathbf{5 . 5}$ remained in solution (Scheme 4.2). The gel was allowed to stand for another $30 \mathrm{~min}$ to ensure complete polymerization. 


\section{Removal of failure sequences and other impurities}

To the ODN-polymer conjugate $\mathbf{5 . 6}$ in the round-bottomed flask was added $3 \mathrm{~mL}$ water. The content was gently shaken at rt overnight. The supernatant, which contained the failure sequences $\mathbf{5 . 5}$ and other impurities, was removed with a pipette. The gel was further washed with water $(2 \mathrm{~mL} \times 3 ; 2 \mathrm{~h}$ each time $)$. The supernatant and the washes were combined and evaporated to dryness. The residue was dissolved in $130 \mathrm{~mL}$ water, of which $20 \mathrm{~mL}$ was injected into HPLC to generate trace in Figure 5.2.

\section{Cleavage of full-length ODN from polymer and releasing the $5^{\prime}$-phosphate group}

The gel in the round-bottomed flask was dried under vacuum. Dry DMF (2 mL) was added via a pipette, which was followed by HF-pyridine complex $(60 \mathrm{~mL})$. The mixture was shaken gently under nitrogen for $5 \mathrm{~h} . \mathrm{Me}_{3} \mathrm{SiOMe}(500 \mathrm{~mL})$ was then added. After shaking for 15 min, the supernatant was transferred to Eppendorf tubes. The gel was extracted with water ( $2 \mathrm{~mL} 63$ at $\mathrm{rt} ; 12 \mathrm{~h}, 2 \mathrm{~h}, 2 \mathrm{~h}$, respectively). The supernatant and the extracts were evaporated to dryness in a SpeedVac vacuum concentrator and were combined to give ODN 5.7 (Scheme 5.2). To deprotect the 5'-phosphate group of 5.7, concentrated $\mathrm{NH}_{4} \mathrm{OH}(\sim 28 \%, 100 \mathrm{~mL})$ was added. After a short vortex, the mixture was heated to $80^{\circ} \mathrm{C}$ for $30 \mathrm{~min}$. After cooling to $\mathrm{rt}, n$ - $\mathrm{BuOH}(900 \mathrm{~mL})$ was added. The mixture was vortexed for $30 \mathrm{~s}$ and then centrifuged at $14.5 \mathrm{~K}$ for $5 \mathrm{~min}$. The supernatant was removed. The residue was further dried shortly in a SpeedVac. The ODN 5.8 was dissolved in $130 \mathrm{~mL}$ water, of which $20 \mathrm{~mL}$ was injected into HPLC to generate the profile (Figure 5.3). The recovery yield of the purification process was estimated to be $55 \%$ by comparing the area of the peak in Figure 5.3 at 19 min with the peak area in Figure 5.1 at $62 \mathrm{~min}$. 
MALDI-TOF mass spectrum of ODN 5.8: calculated for $[\mathrm{M}-2 \mathrm{H}+\mathrm{Na}]^{-}$ $\mathrm{C}_{194} \mathrm{H}_{247} \mathrm{~N}_{67} \mathrm{NaO}_{125} \mathrm{P}_{20}$ 6159.0, found 6159.8.

\section{Synthesis and purification of the 61-mer ODN 5.10}

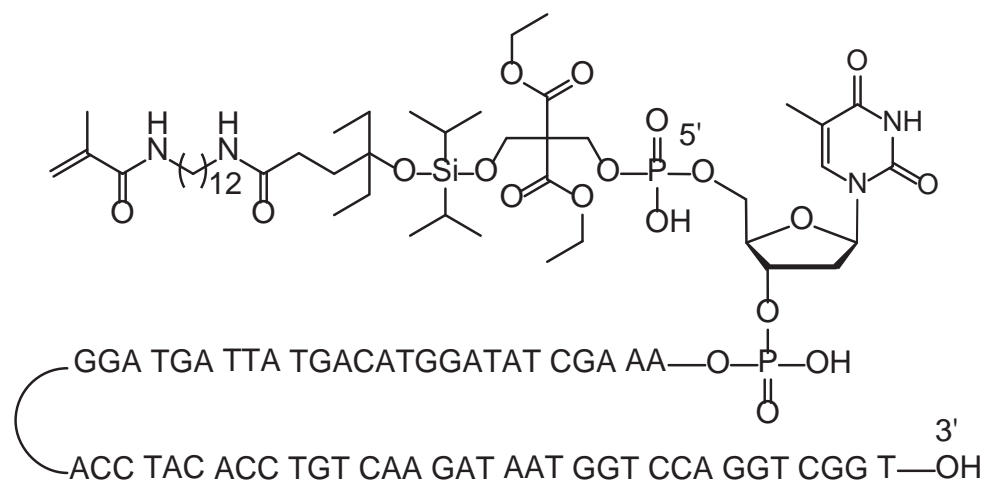

5.9

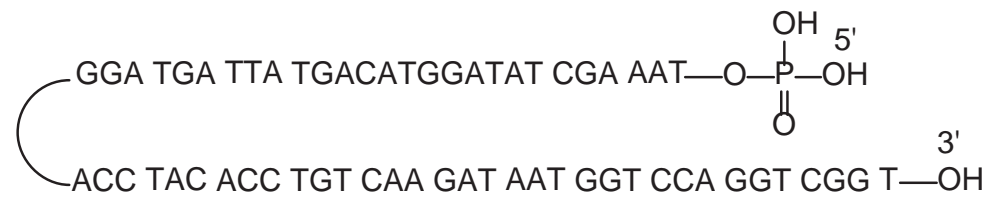

5.10

For the solid phase synthesis, cleavage and deprotection of 5.9, the procedure for

5.4 was followed except for the following modifications. CPG with a pore size of $2000 \AA$ instead of $1000 \AA$ was used. Before synthesis, the CPG was manually capped with $\mathrm{Pac}_{2} \mathrm{O}$ for $20 \mathrm{~min}$ on the synthesizer. In the synthetic cycle, a 25 -second waiting step was added after each delivery of coupling reagents (phosphoramidite and tetrazole) to the synthesis column. An additional capping step was added, and after each capping step, a 50-second waiting step was added. The catching by polymerization procedure was exactly the same as described for 5.4. However, for RP HPLC analysis of 5.10, buffer A that contained 10\% urea was used. 

groups

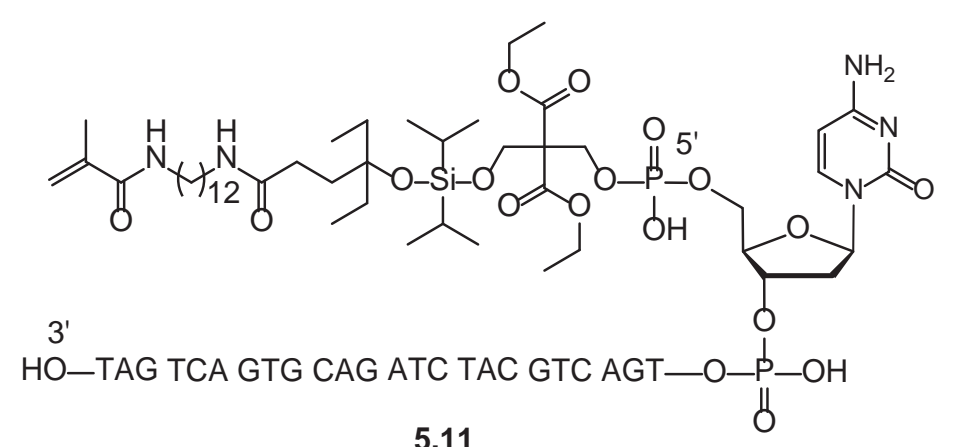

5.11

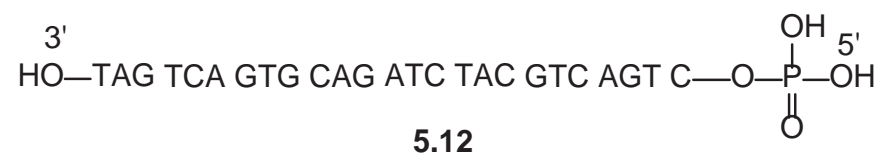

For the solid phase synthesis, cleavage and deprotection of 5.11, the procedure for 5.4 was followed except for the following modifications. The 5'-DMTr-protected 2cyanoethyl phosphoramidite monomers $\mathrm{Bz}-\mathrm{dA}, i-\mathrm{Bu}-\mathrm{dG}, \mathrm{Ac}-\mathrm{dC}$ and $\mathrm{dT}$ were used. The capping agents were replaced with $\mathrm{THF} / \mathrm{pyridine} / \mathrm{Ac}_{2} \mathrm{O}$. After cleaving the ODN from CPG with concentrated $\mathrm{NH}_{4} \mathrm{OH}$ at $\mathrm{rt}$, the solution was heated to $55^{\circ} \mathrm{C}$ for $8 \mathrm{~h}$ in a tightly capped vial. The catching by polymerization procedure was exactly the same as described for $\mathbf{5 . 4}$. 


\section{Acknowledgements}

Financial support from US NSF (CHE-0647129), Michigan Universities Commercialization Initiative, MTU Research Excellence Fund (REF-TC), MTU Chemistry Department, MTU Biotech Research Center, and The Royal Thai Government Scholarship (S. F.); the assistance from Mr. Jerry L. Lutz (NMR), Mr. Shane Crist (computation), and Mr. Dean W. Seppala (electronics); and an NSF equipment grant (CHE9512445) are all gratefully acknowledged.

[Yuan, Y.; Fueangfung, S.; Lin, X.; Pokharel, D.; Fang, S., Synthetic 5'-phosphorylated oligodeoxynucleotide purification through catching full-length sequences by polymerization. RSC Advances 2012, 2 (7), 2803-2808.] - Reproduced by permission of The Royal Society of Chemistry

http://pubs.rsc.org/en/Content/ArticleLanding/2012/RA/C2RA01357F\#!divAbstract 


\section{References}

1. Bates, P. J.; Laber, D. A.; Miller, D. M.; Thomas, S. D.; Trent, J. O., Discovery and development of the G-rich oligonucleotide AS1411 as a novel treatment for cancer. Experimental and Molecular Pathology 2009, 86 (3), 151-164.

2. De Rosa, G.; La Rotonda, M. I., Nano and Microtechnologies for the Delivery of Oligonucleotides with Gene Silencing Properties. Molecules 2009, 14 (8), 2801-2823.

3. Zhou, C.; Chattopadhyaya, J., The synthesis of therapeutic locked nucleos(t)ides. Current Opinion Drug Discovery Development, 2009, 12, 876-898.

4. Khati, M.; Clin. J., The future of aptamers in medicine. Journal of Clinical Pathology, 2010, 63, 480-487.

5. Singh, Y.; Murat, P.; Defrancq, E., Recent developments in oligonucleotide conjugation. Chemical Society Reviews 2010, 39 (6), 2054-2070.

6. Zhang, D. Y.; Seelig, G., Dynamic DNA nanotechnology using stranddisplacement reactions. Nature Chemistry 2011, 3 (2), 103-113.

7. Matsuda, A., Development of highly nuclease-resistant chemically-modified oligonucleotides. Journal of Pharmaceutical Society Japan, 2011, 131, 285-298.

8. Yang, X.; Li, N.; Gorenstein, D. G., Strategies for the discovery of therapeutic aptamers. Expert Opinion on Drug Discovery, 2011, 6, 75-87.

9. Beaucage, S. L.; Bergstrom, D. E.; Glick, G. D.; Jones, R. A. In Current Protocols in Nucleic Acid Chemistry, John Wiley \& Sons, Inc.: 2000. 
10. Pearson, W. H.; Berry, D. A.; Stoy, P.; Jung, K.-Y.; Sercel, A. D., Fluorous Affinity Purification of Oligonucleotides. The Journal of Organic Chemistry 2005, 70 (18), 71147122.

11. Beller, C.; Bannwarth, W., Noncovalent Attachment of Nucleotides by FluorousFluorous Interactions: Application to a Simple Purification Principle for Synthetic DNA Fragments. Helvetica Chimica Acta 2005, 88 (1), 171-179.

12. Fang, S.; Bergstrom, D. E., Reversible 5'-end biotinylation and affinity purification of synthetic RNA. Tetrahedron Letters 2004, 45 (43), 7987-7990.

13. Fang, S.; Bergstrom, D. E., Fluoride-cleavable biotinylation phosphoramidite for 5'-end-labeling and affinity purification of synthetic oligonucleotides. Nucleic Acids Research 2003, 31 (2), 708-715

14. Fang, S.; Bergstrom, D. E., Reversible Biotinylation Phosphoramidite for 5`-EndLabeling, Phosphorylation, and Affinity Purification of Synthetic Oligonucleotides. Bioconjugate Chemistry 2003, 14 (1), 80-85.

15. Sproat, B. S.; Rupp, T.; Menhardt, N.; Keane, D.; Beijer, B., Fast and simple purification of chemically modified hammerhead ribozymes using a lipophilic capture tag. Nucleic Acids Research 1999, 27 (8), 1950-1955.

16. Fang, S.; Fueangfung, S., Scalable Synthetic Oligodeoxynucleotide Purification with Use of a Catching by Polymerization, Washing, and Releasing Approach. Organic Letters 2010, 12 (16), 3720-3723.

17. Fang, S.; Fueangfung, S.; Lin, X.; Zhang, X.; Mai, W.; Bi, L.; Green, S. A., Synthetic oligodeoxynucleotide purification by polymerization of failure sequences. Chemical Communications 2011, 47 (4), 1345-1347. 
18. Modrich, P.; Lehman, I. R., Deoxyribonucleic Acid Ligase: A STEADY STATE KINETIC ANALYSIS OF THE REACTION CATALYZED BY THE ENZYME FROM ESCHERICHIA COLI. Journal of Biological Chemistry 1973, 248 (21), 7502-7511.

19. Sambrook, J.;Russell, D. W., Molecular Cloning: A Laboratory Manual, Cold Spring Harbor Press, Cold Spring Harbor, New York: 2001.

20. Fritz, H.-J. In DNA Cloning: A Practical Approach, ed. D. M. Glover, IRL Press, Oxford, 1985, 1, 151-163.

21. Barany, F., The ligase chain reaction in a PCR world. PCR Methods and Applications, 1991, 1, 5-16.

22. Horn, T.; Urdea, M. S., A chemical 5'-phosphorylation of oligodeoxyribonucleotides that can be monitored by trityl cation release. Tetrahedron Letters 1986, 27 (39), 4705-4708.

23. Guzaev, A.; Salo, H.; Azhayev, A.; Lönnberg, H., A new approach for chemical phosphorylation of oligonucleotides at the 5'-terminus. Tetrahedron 1995, 51 (34), 93759384. 


\section{Chapter 6}

\section{Future Research Plan}

ODN purification technology has become vital for many research applications including ODN drug development and biological studies. In preceding chapters, we described our technology which is to purify synthetic ODN including catching the fulllength sequence and catching failure sequences. The principle is to use polymerization reaction to purify ODN, which is low cost and simple. Despite the fact that the technology is successful, there are some elements that we can improve the methods and extend this technology for the future.

\subsection{User-friendly cleavable linker for ODN Purification by Catching Full-length Sequence}

The phosphoramidite monomer 3.1, which was described in chapter 3, has diisopropyl silyl acetal group as a cleavable linker. After polymerization and impurities removal, purified ODN was released from the gel under anhydrous conditions using HFpyridine. Although the results from the purification method were satisfactory, HF-pyridine might cause a problem when doing large-scale ODN purification because HF is corrosive and very toxic. In addition, cleavage will not be efficient if the gel still contains water. Thus, the procedure requires the step to remove water from the gel.

To simplify the method, the other phosphoramidite monomer is proposed in Figure 6.1. The phosphoramidite $\mathbf{6 . 1}$ has a modified DMTr group as a cleavable linker. This can avoid using HF-pyridine. Unlike diisopropyl silyl acetal group, this modified DMTr can 
be cleaved using weak acid (eg. $\mathrm{CH}_{3} \mathrm{COOH}$ ). Also, it does not need to remove water from the gel before cleaving. The purification process is proposed in scheme 6.1. The compound 6.1 would be coupled in the last cycle of ODN synthesis to get crude ODN (6.2 and 6.3). Then, 6.2 would be incorporated in the gel during polymerization. Once impurities are removed, weak acidic conditions would be used to cleave $\mathbf{6 . 4}$ to get pure $\mathbf{6 . 5}$.

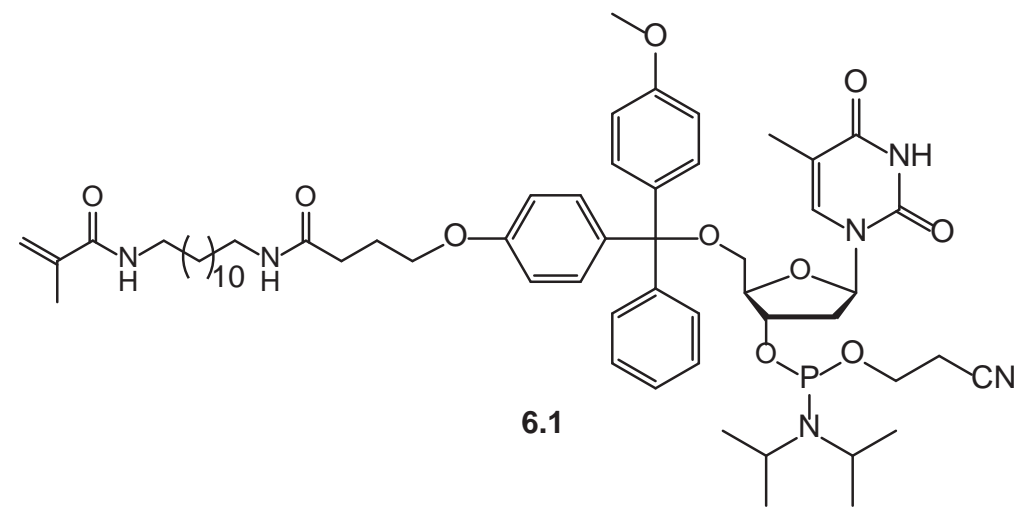

Figure 6.1. Phosphoramidite 6.1 


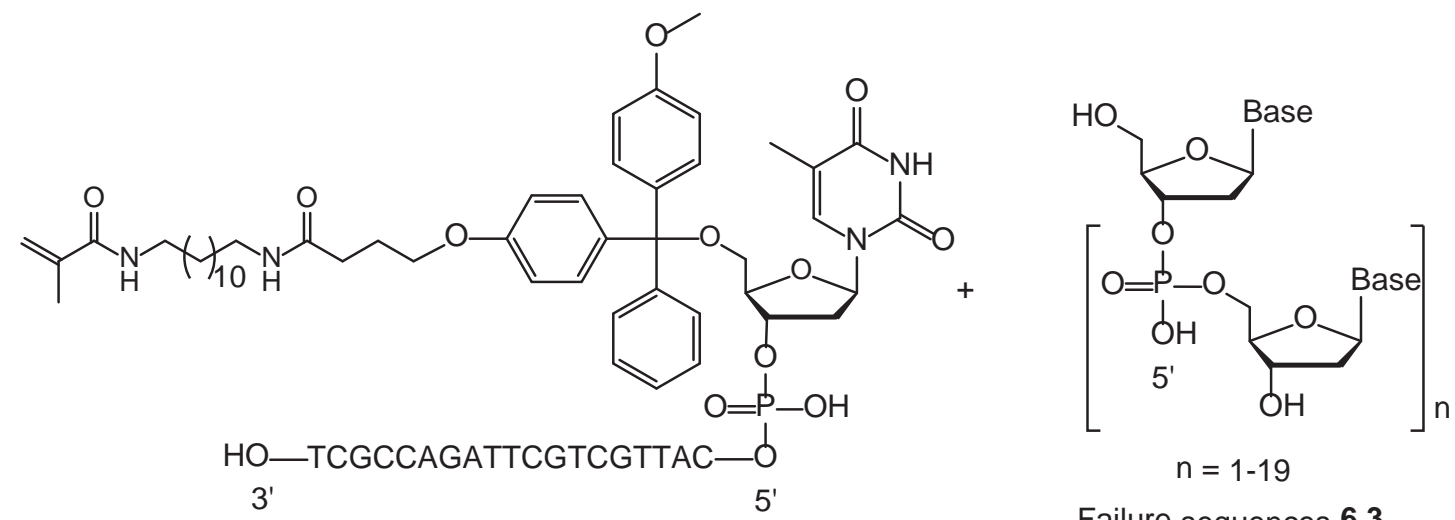

Full-length sequence $\mathbf{6 . 2}$

Failure sequences 6.3

1. $N, N$-Dimethylacrylamide $N, N$ '-methylenebis(acrylamide) $\left(\mathrm{NH}_{4}\right)_{2} \mathrm{~S}_{2} \mathrm{O}_{8}$, TMEDA, $\mathrm{H}_{2} \mathrm{O}$, rt, $1 \mathrm{~h}$
2. Wash with water to remove 6.3 and other impurities

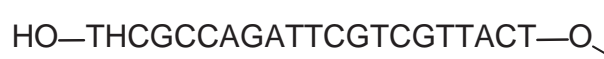

3'
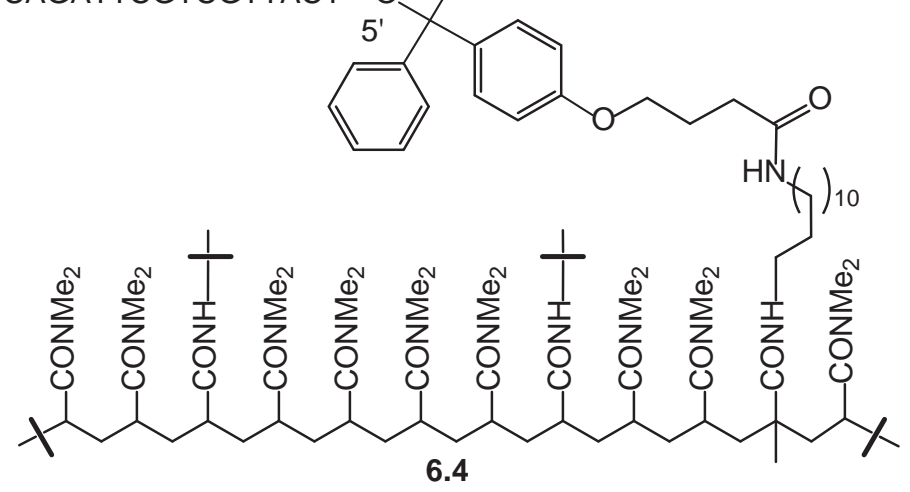

$\mathrm{CH}_{3} \mathrm{COOH}$ solution

HO_TCGCCAGATTCGTCGTTACT-OH 6.5

3'

+ Polymer

Scheme 6.1. Proposed ODN purification using phosphoramidite 6.1 


\subsection{Detailed Studies on ODN Purification by Capping Failure Sequences}

In chapter 4 , the phosphoramidite 4.1 was introduced for catching failure sequences. To continue further studies, the other three capping phosphoramidites would be designed to test capping efficiency and optimal time (Figure 6.2). The hypothesis is that ether oxygen atom in each compound can increase the solubility in organic solvent (eg. acetonitrile). Therefore, their reaction kinetics while capping might be better than 4.1.
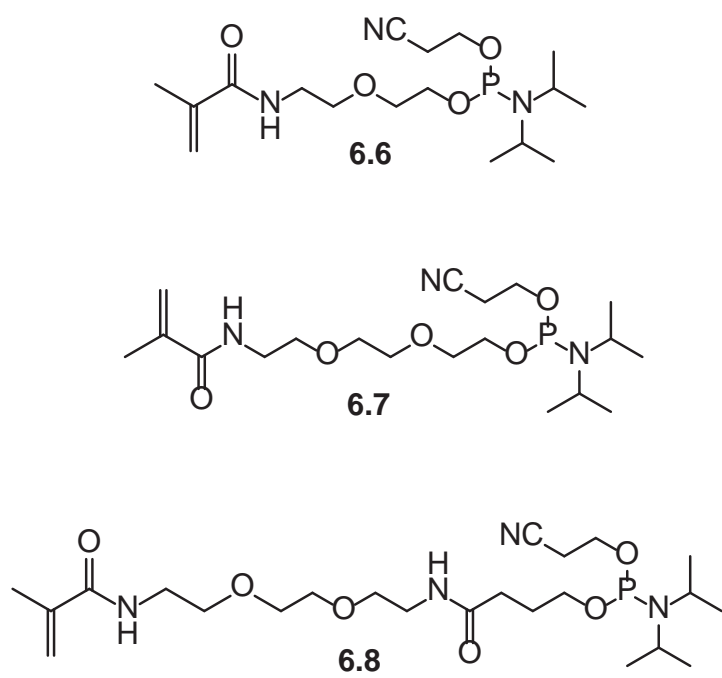

\section{Figure 6.2. New polymerizable capping phosphoramidites}

Moreover, the detailed studies will also be focusing on diffusion speed and extraction efficiency of ODN from gel using different cross-linking ratio, purification of long sequence ODN, larger scale ODN purification, and polymerization of failure sequences in air. Finally, purified ODN will be tested for the stability under radical polymerization by complete enzymatic ODN digestion. All nucleosides from the digestion will be analyzed by RP HPLC. 


\subsection{Synthetic Peptide Purification using Polymerization Approach}

Since synthetic peptides are beneficial to many research area, such as clinical trials, therapeutic studies, and molecular biology, it is undeniable that peptide purification is essential for them. Therefore, purification methodology will be applied to synthetic peptides. In this case, both methods (Catching full-length peptide and catching failure sequences) will be planned for peptide purification.

For catching full-length peptide, compound 6.9 which consists of reactive $p$ nitrophenyl carbonate, an acid-labile linker, and polymerizable group will be synthesized (Figure 6.3). This compound will be incorporated in the last cycle of peptide synthesis. Then, full-length peptide will be in the gel by polymerization. Impurities including failure sequences will be removed by washing. Purified peptide will be given by cleaving from the gel. For catching failure sequences, compound $\mathbf{6 . 1 0}$ will be used as a capping agent (Figure 6.4). All failure sequences will be capped with 6.10. Then, polymerization will remove failure sequences and give pure full-length peptide. 


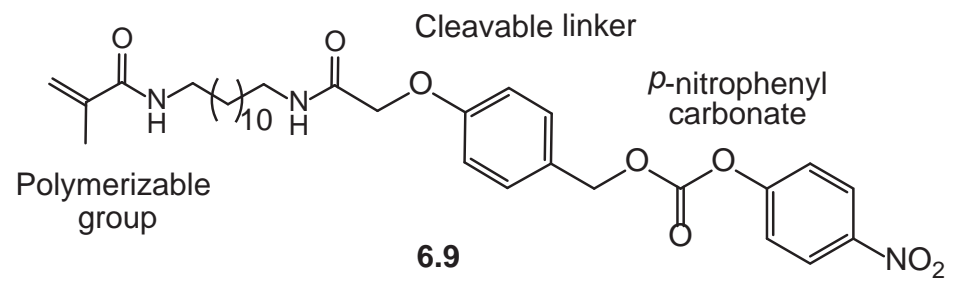

Figure 6.3. Compound 6.9

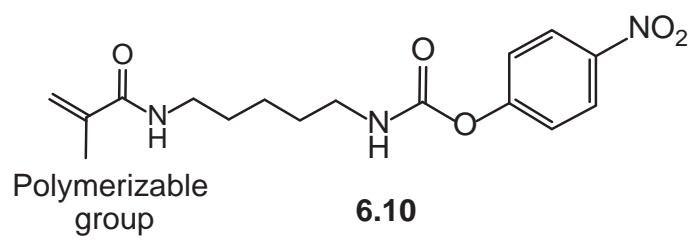

Figure 6.4. Compound 6.10 


\section{Appendix A}

Supporting Information for Chapter 3

Scalable Synthetic Oligodeoxynucleotide Purification with Use of a Catching by Polymerization, Washing, and Releasing Approach 


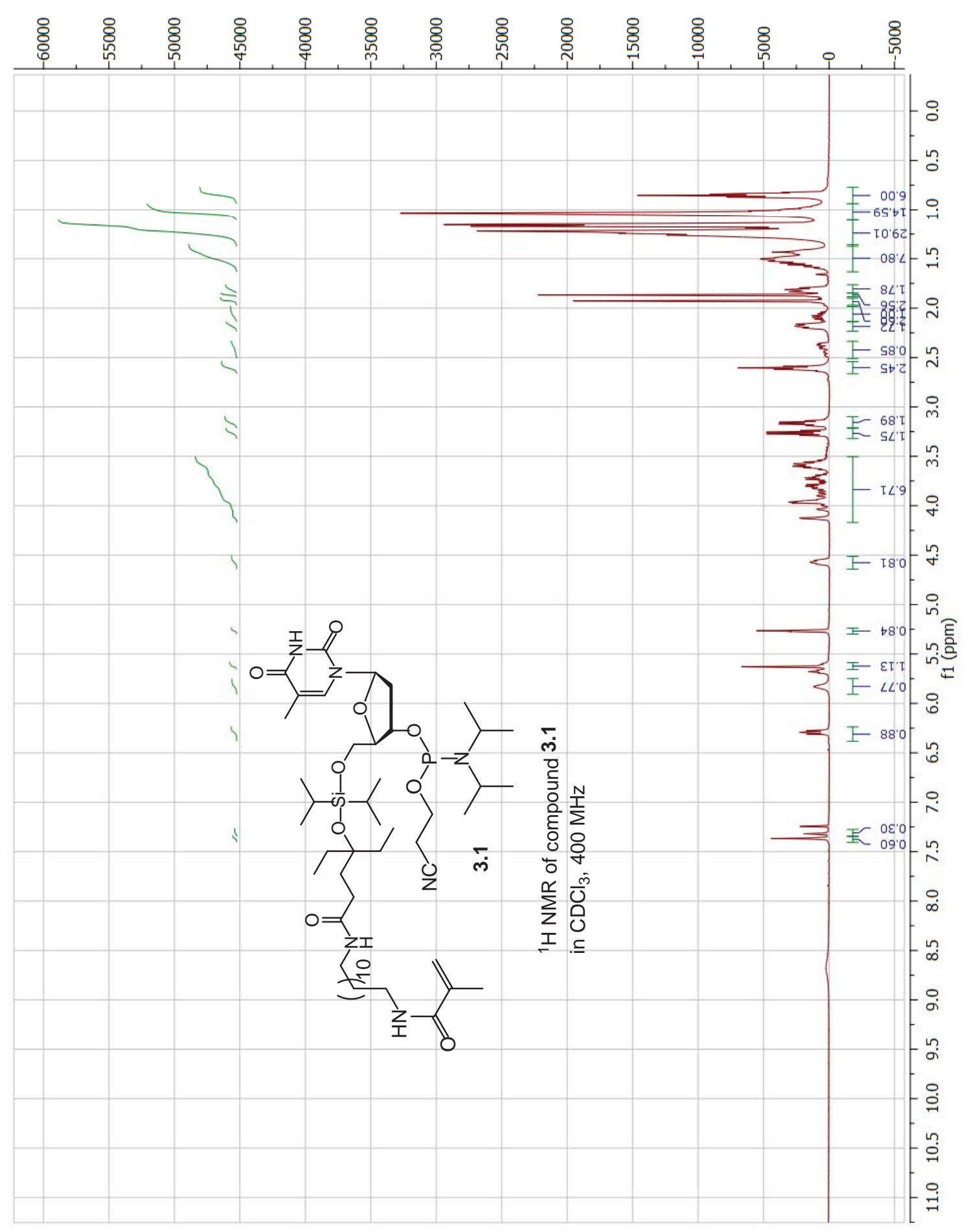

Figure A.1. ${ }^{1} \mathrm{H}-\mathrm{NMR}$ of compound 3.1 


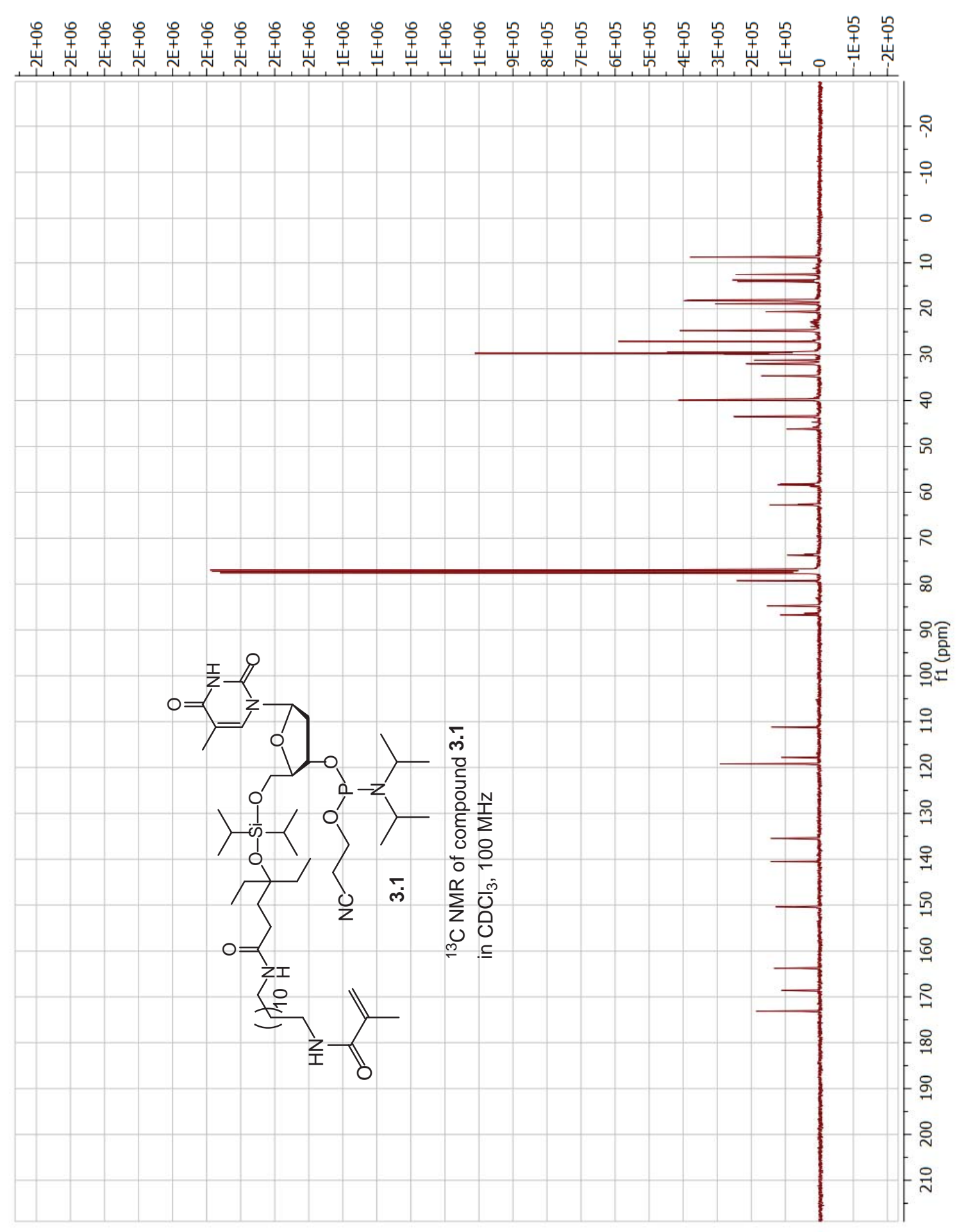

Figure A.2. ${ }^{13} \mathrm{C}-\mathrm{NMR}$ of compound 3.1 


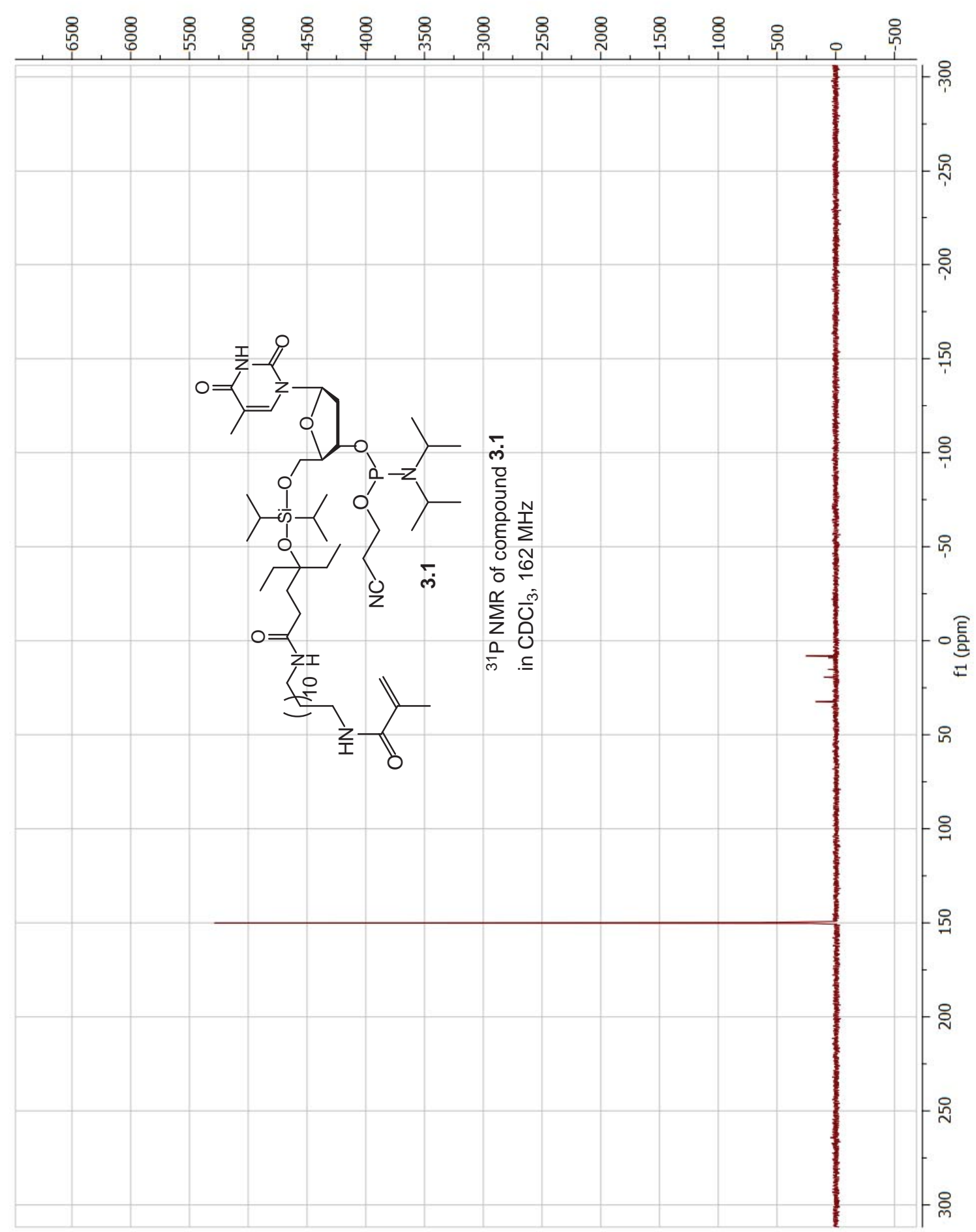

Figure A.3. ${ }^{31} \mathrm{P}-\mathrm{NMR}$ of compound $\mathbf{3 . 1}$ 


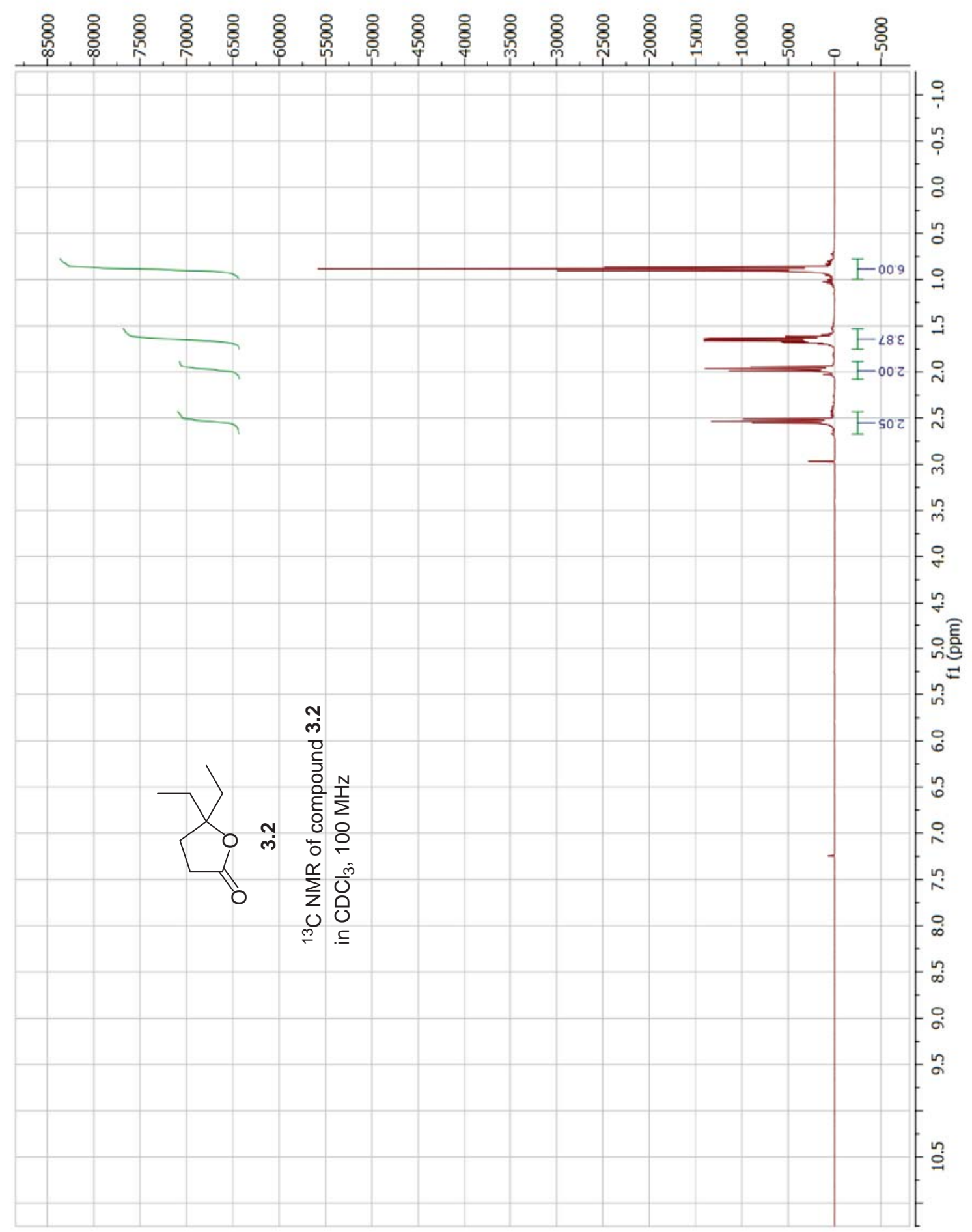

Figure A.4. ${ }^{1} \mathrm{H}-\mathrm{NMR}$ of compound 3.2 


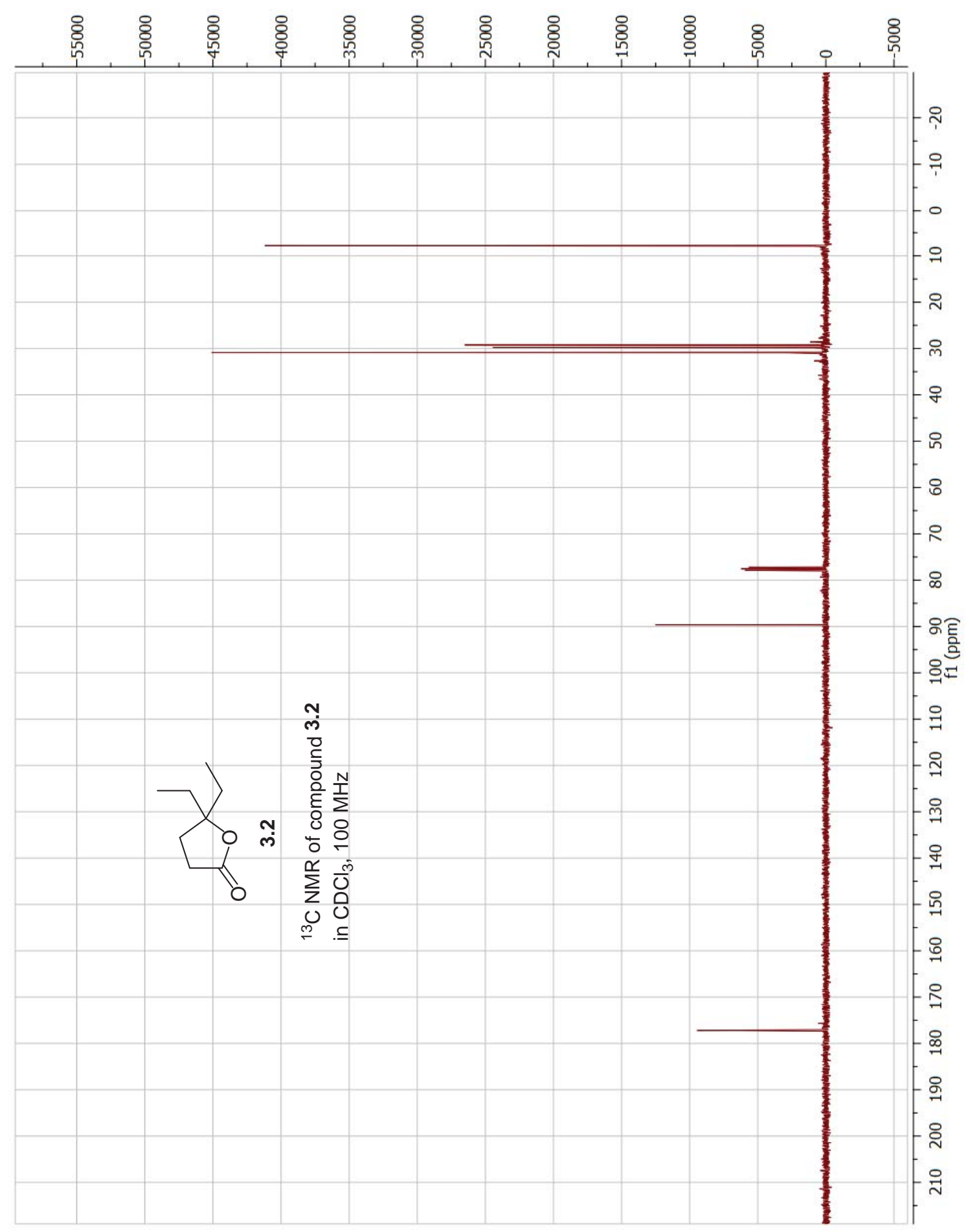

Figure A.5. ${ }^{13} \mathrm{C}-\mathrm{NMR}$ of compound $\mathbf{3 . 2}$ 

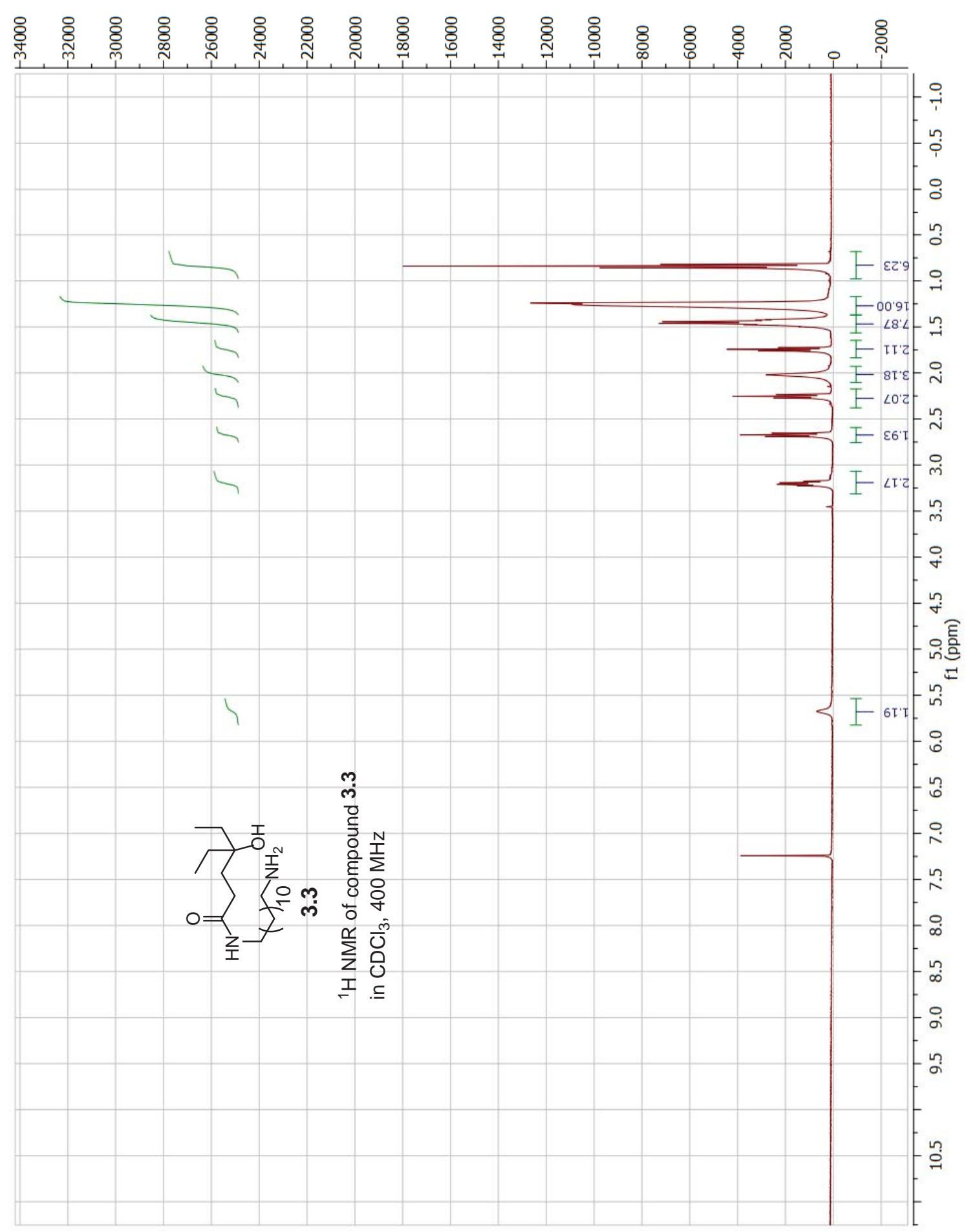

Figure A.6. ${ }^{1} \mathrm{H}-\mathrm{NMR}$ of compound 3.3 


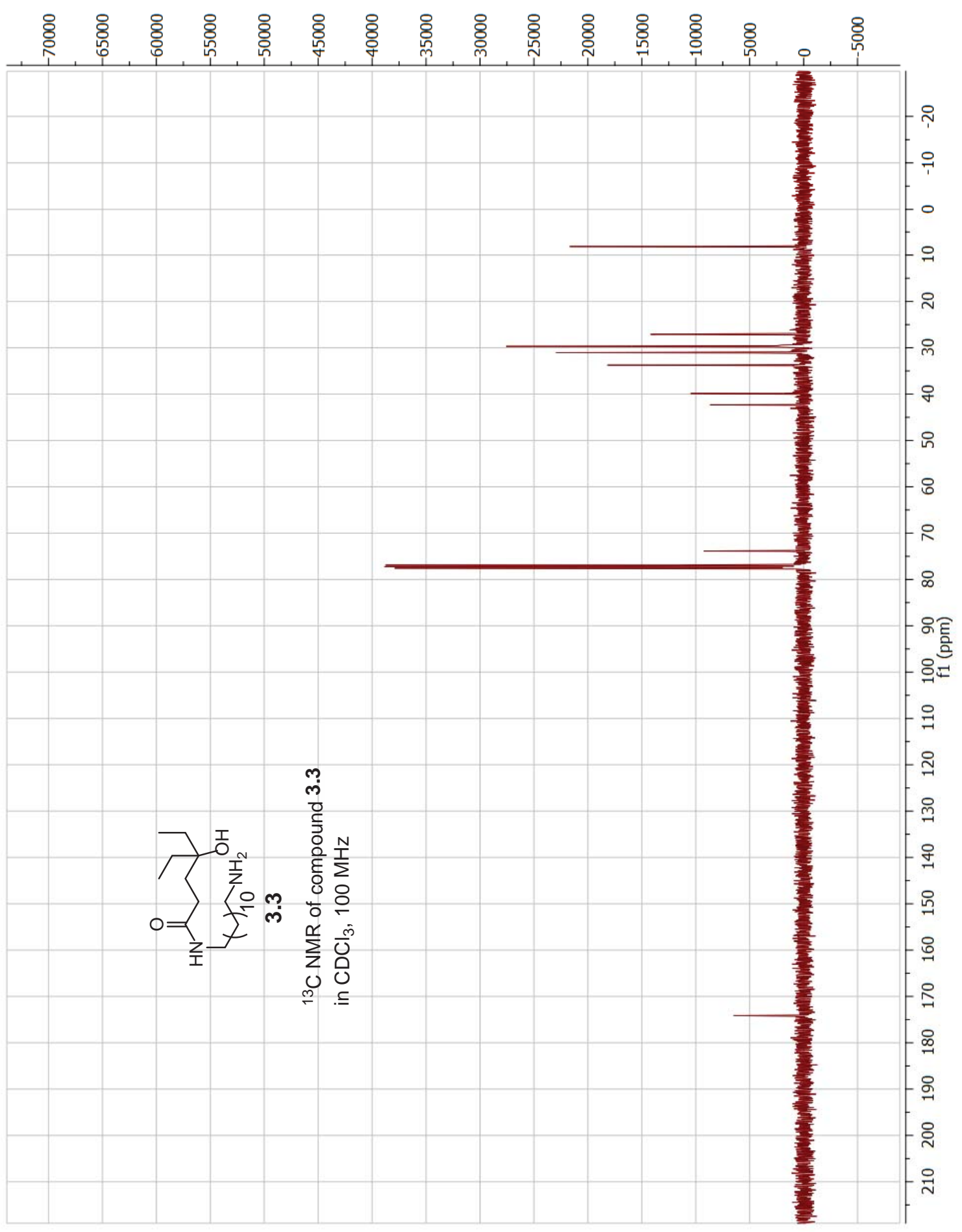

Figure A.7. ${ }^{13} \mathrm{C}-\mathrm{NMR}$ of compound $\mathbf{3 . 3}$ 

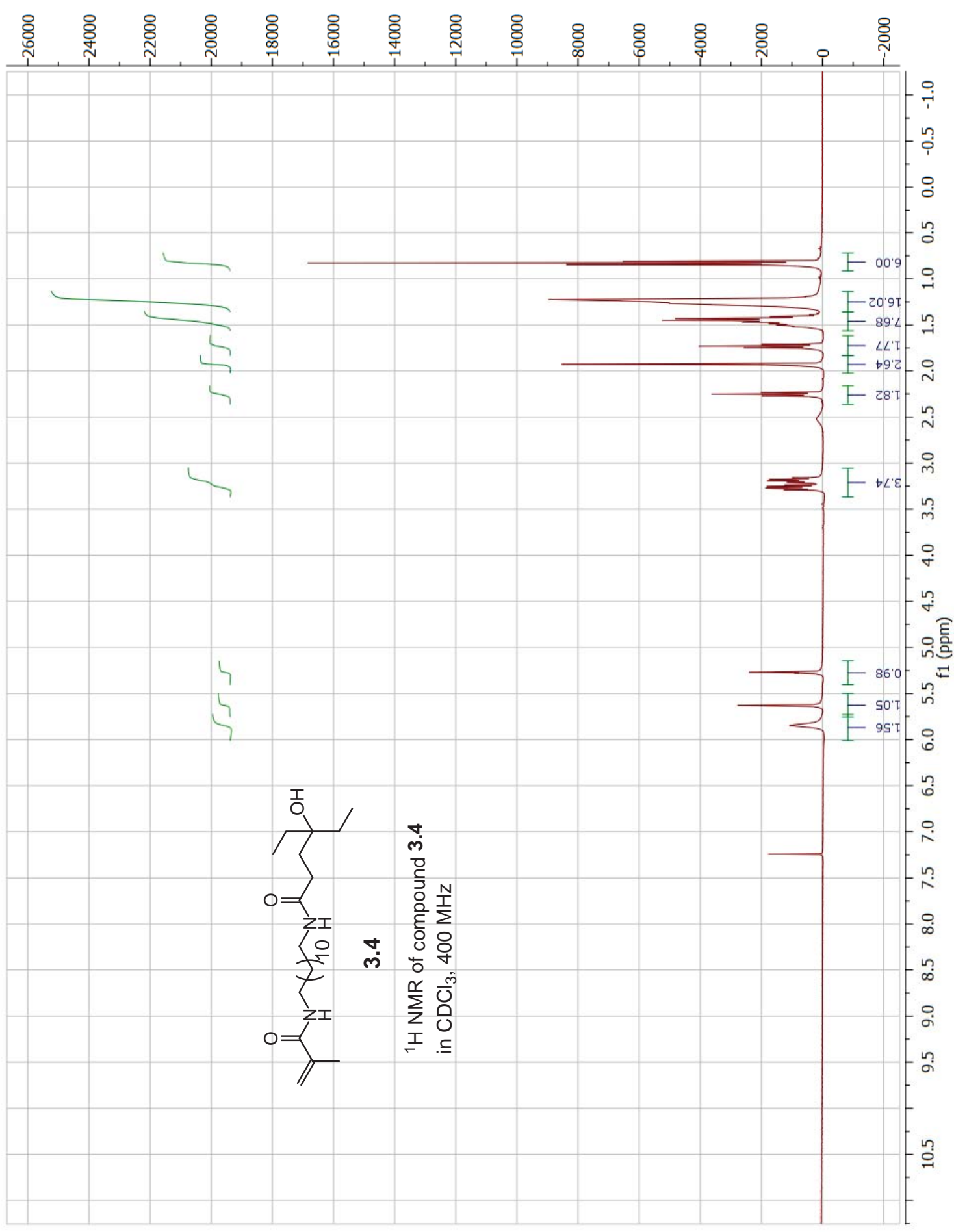

Figure A.8. ${ }^{1} \mathrm{H}-\mathrm{NMR}$ of compound 3.4 


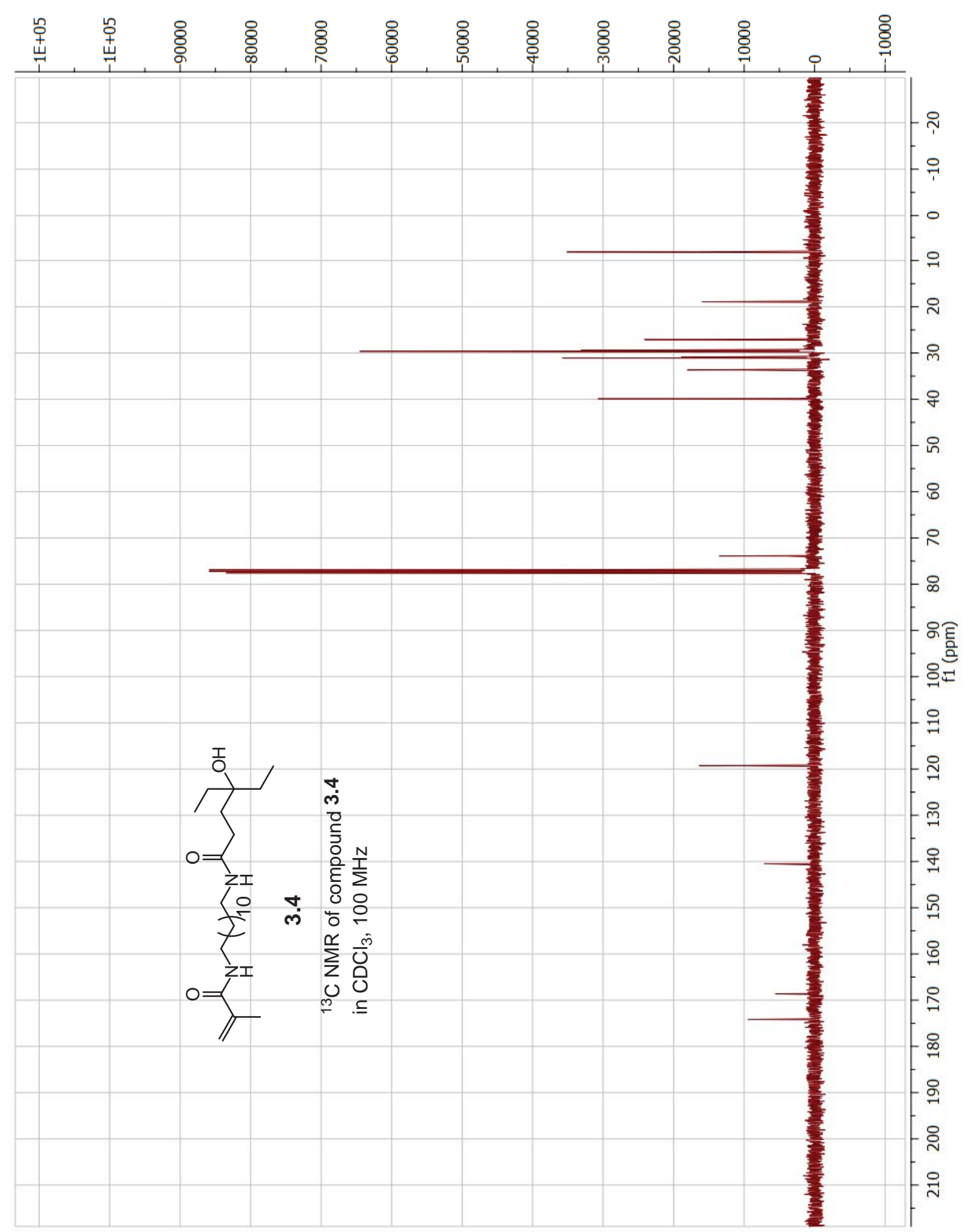

Figure A.9. ${ }^{13} \mathrm{C}-\mathrm{NMR}$ of compound 3.4 


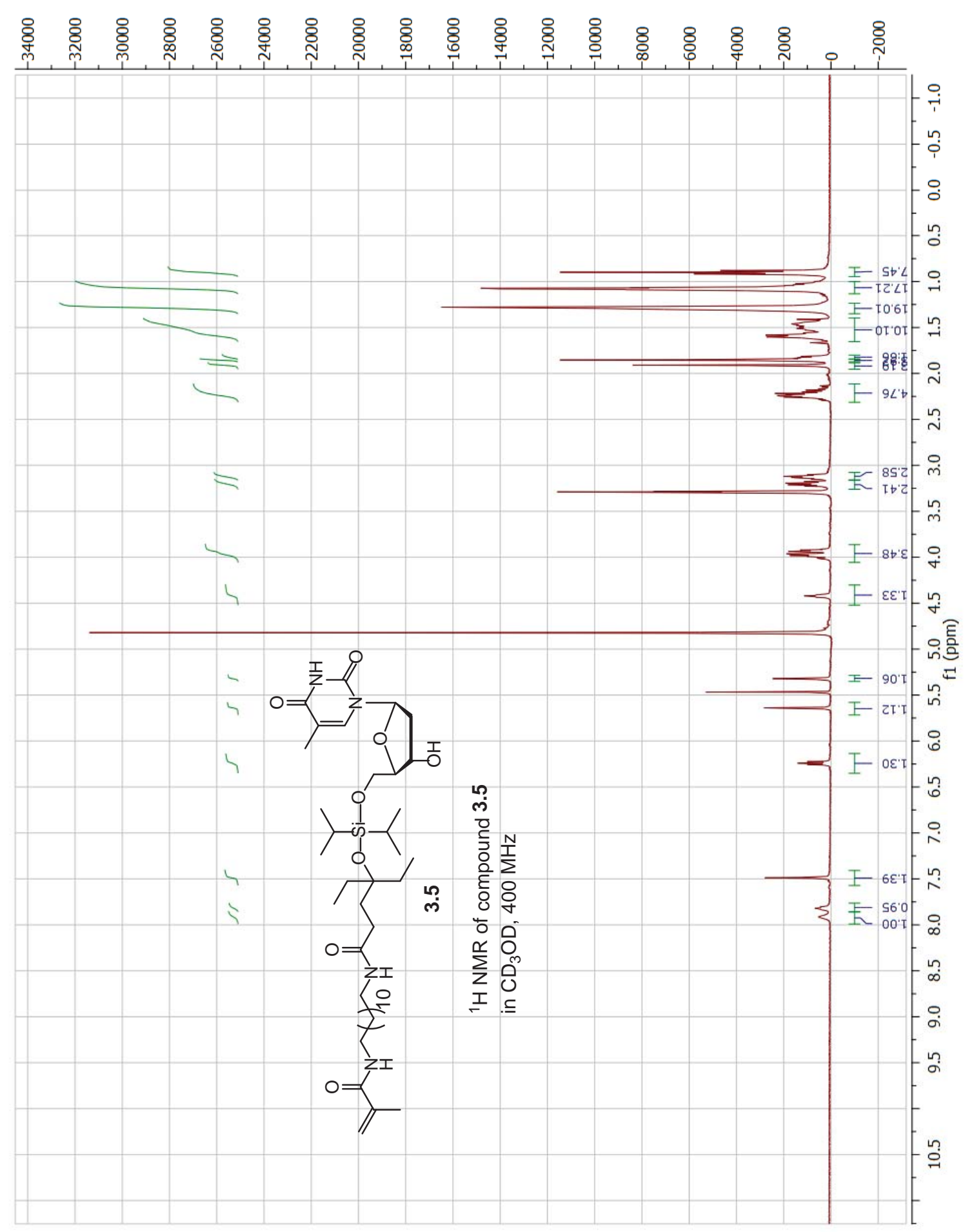

Figure A.10. ${ }^{1} \mathrm{H}-\mathrm{NMR}$ of compound $\mathbf{3 . 5}$ 


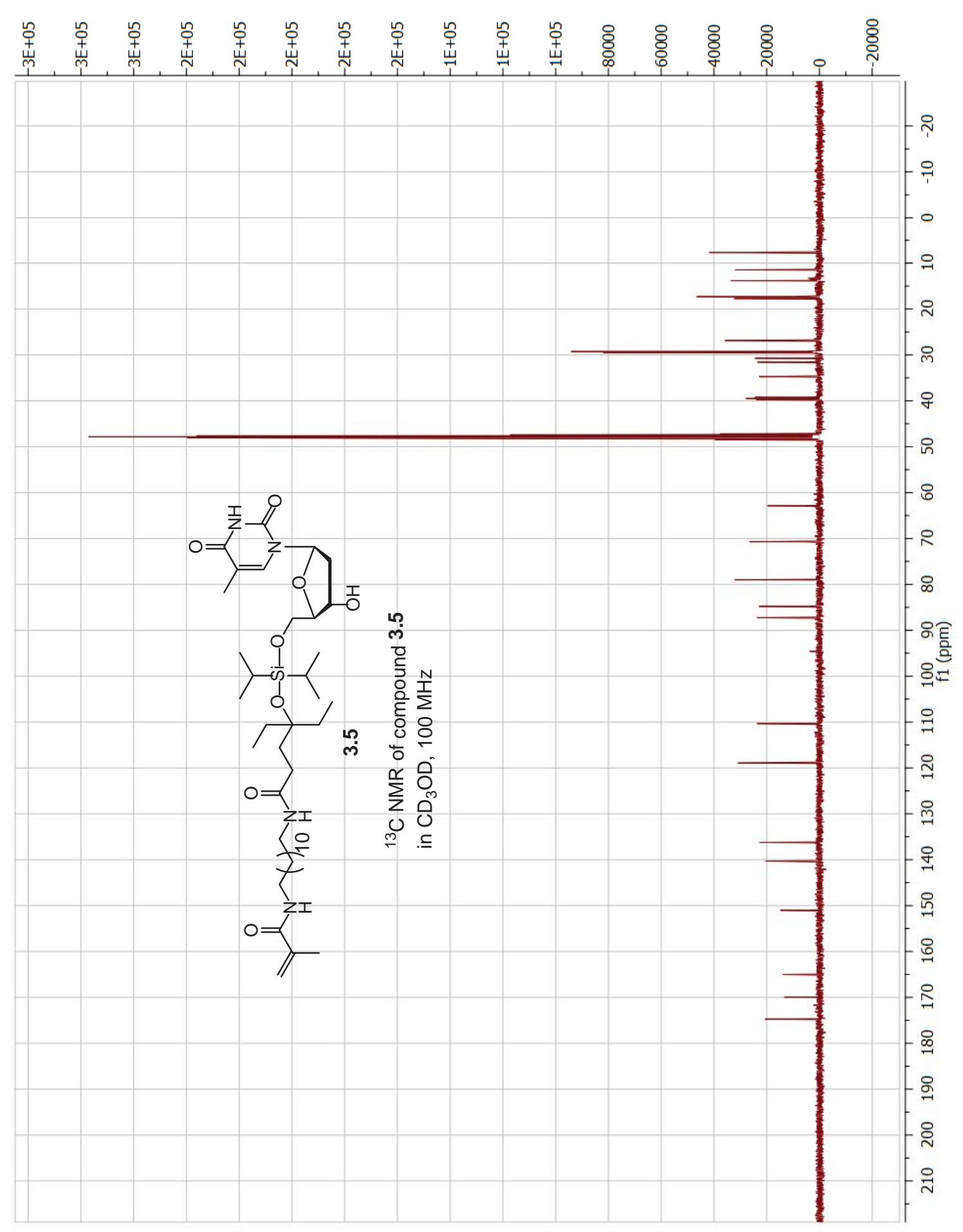

Figure A.11. ${ }^{13} \mathrm{C}-\mathrm{NMR}$ of compound $\mathbf{3 . 5}$ 


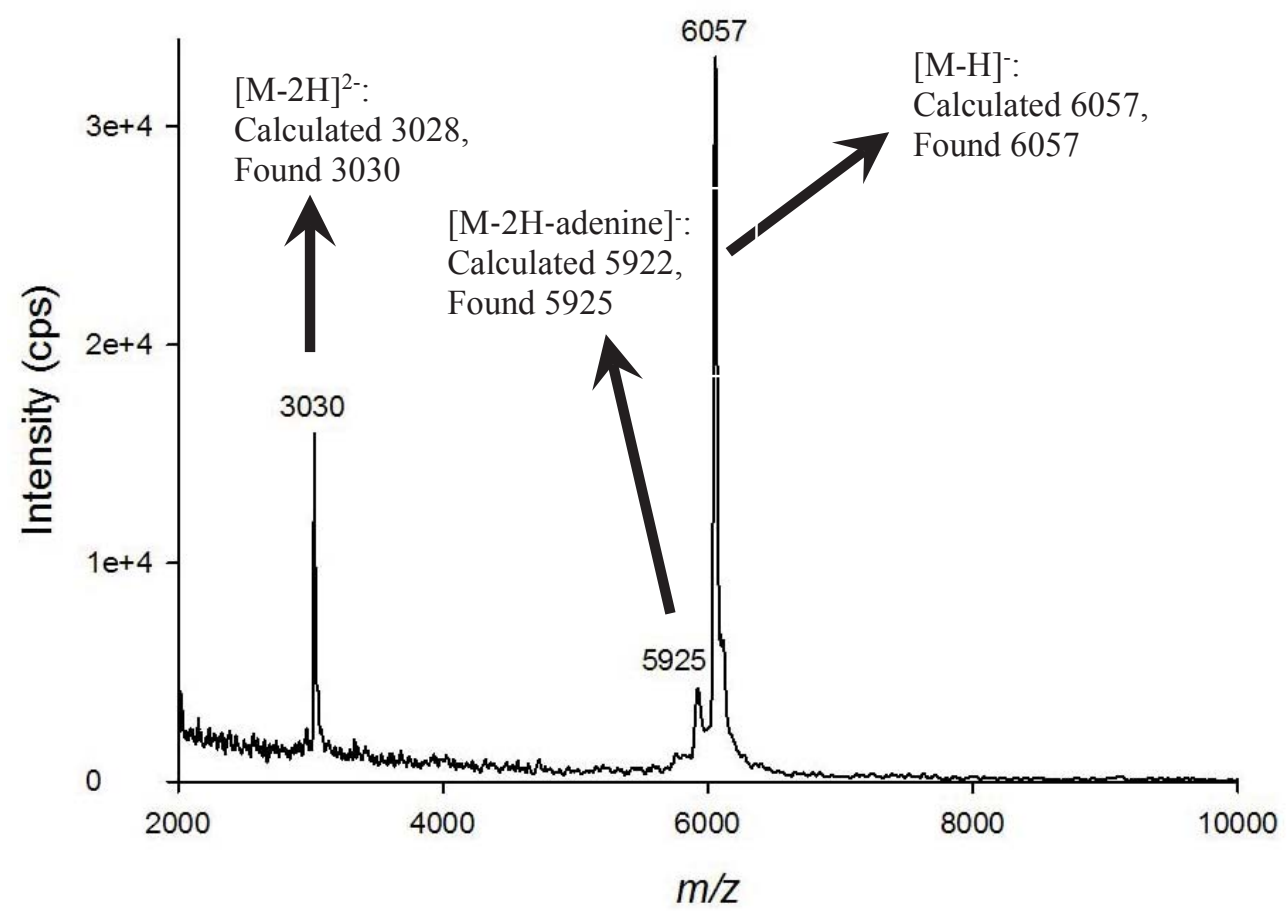

Figure A.12. MALDI-TOF mass spectrum of ODN 3.9 purified using the catching by polymerization, washing and releasing approach 


\section{Appendix B}

Supporting Information for Chapter 4

Synthetic Oligodeoxynucleotide Purification by Polymerization

of Failure Sequences 


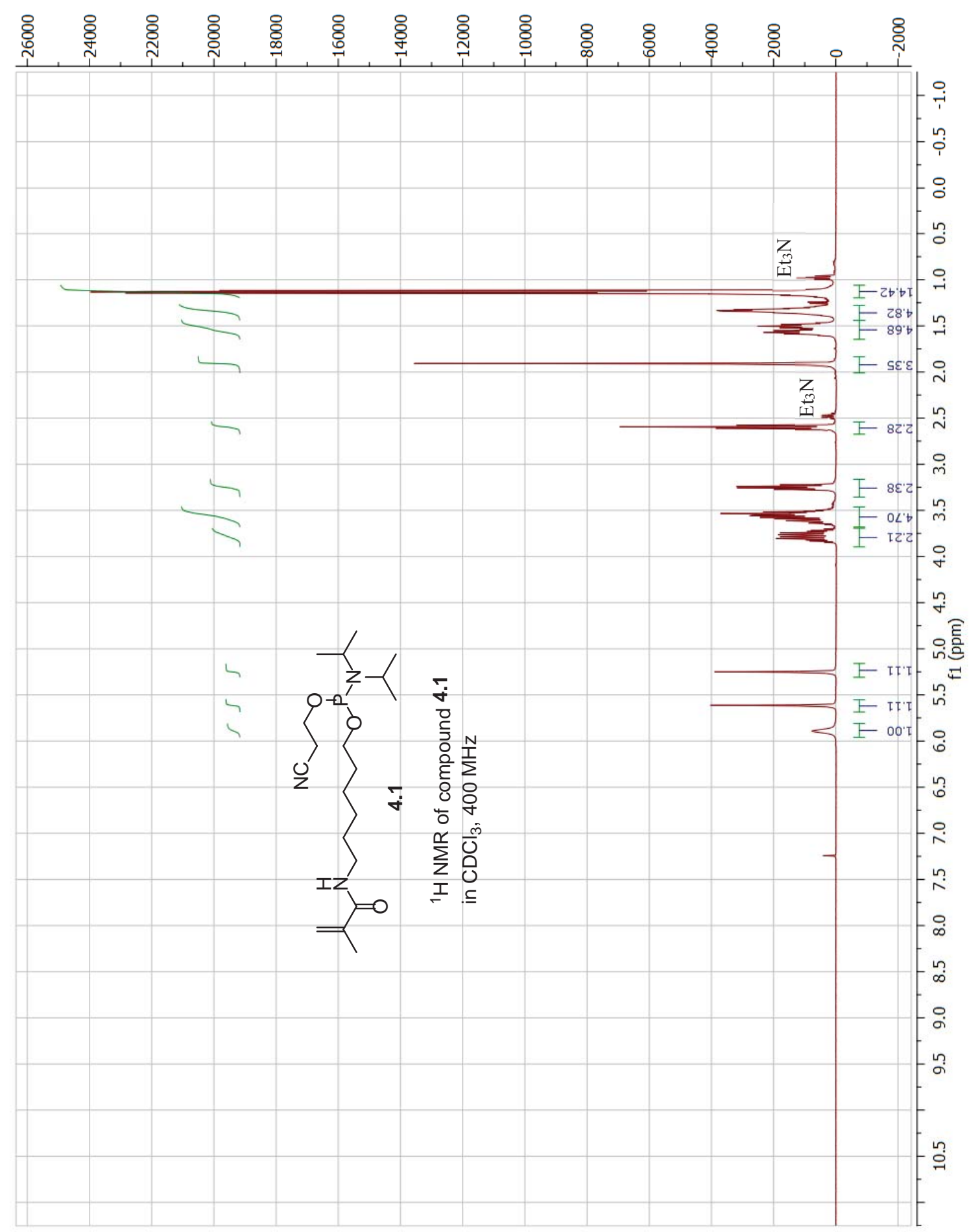

Figure B.1. ${ }^{1} \mathrm{H}-\mathrm{NMR}$ of compound 4.1 


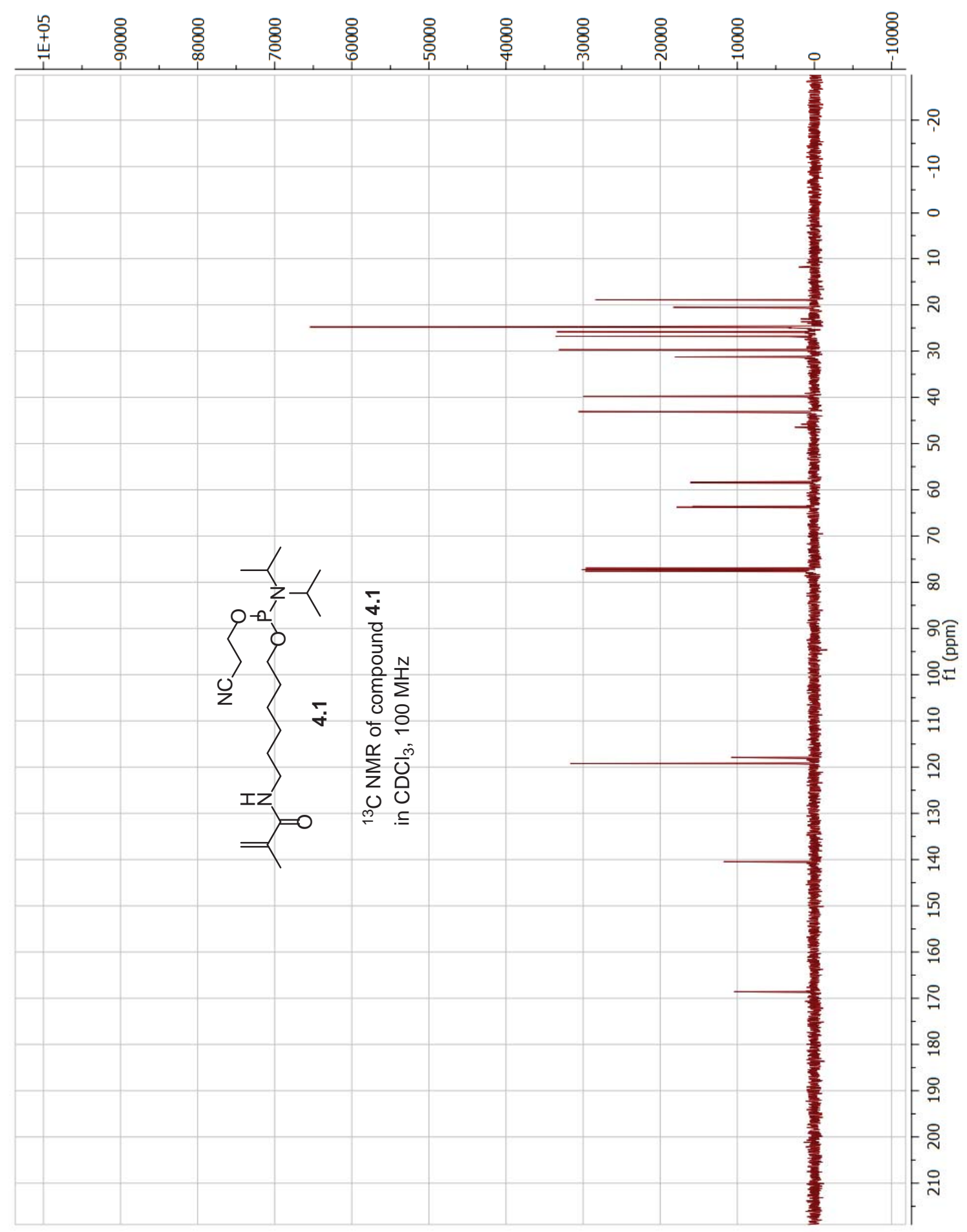

Figure B.2. ${ }^{13} \mathrm{C}-\mathrm{NMR}$ of compound 4.1 


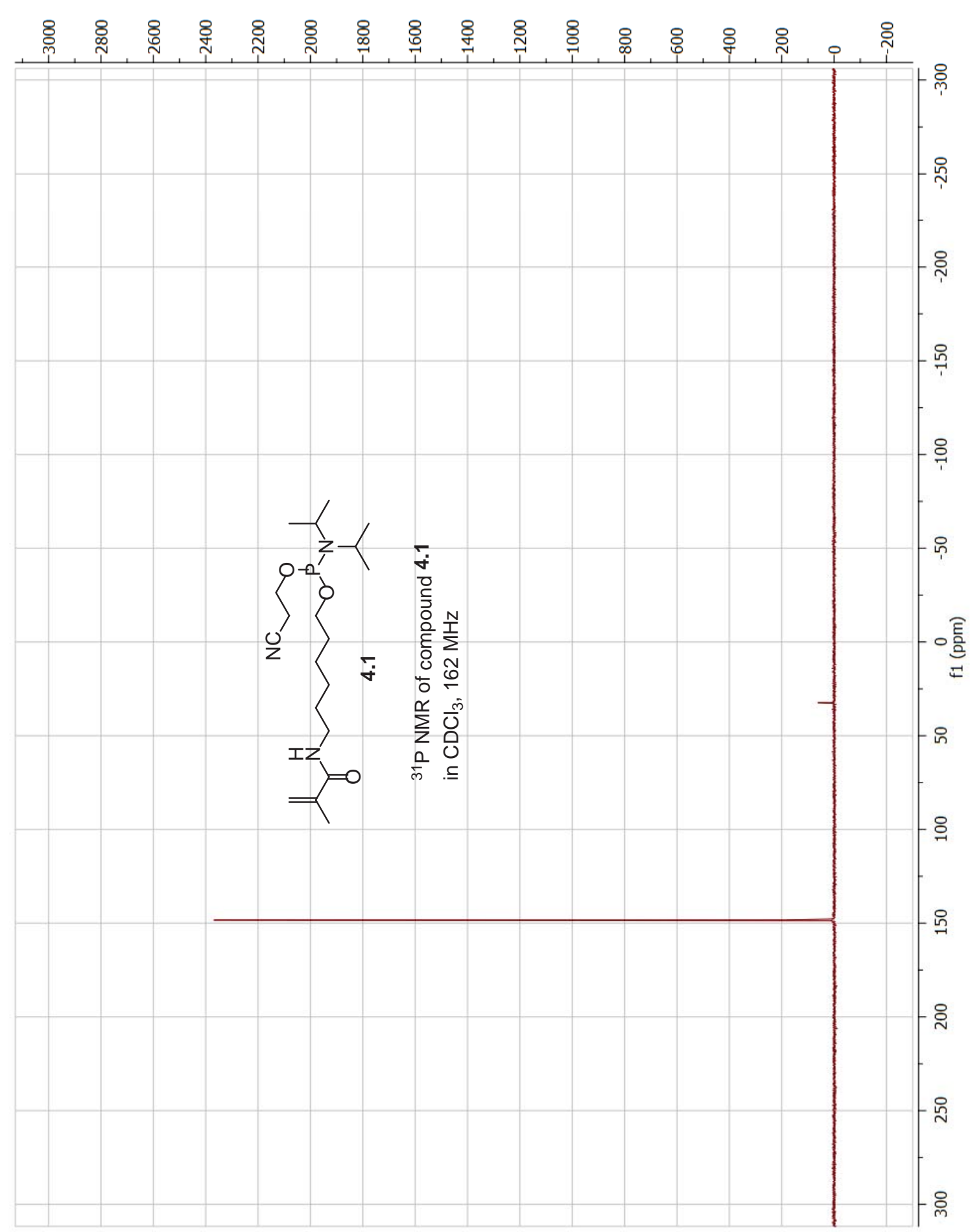

Figure B.3. ${ }^{31} \mathrm{P}-\mathrm{NMR}$ of compound 4.1 


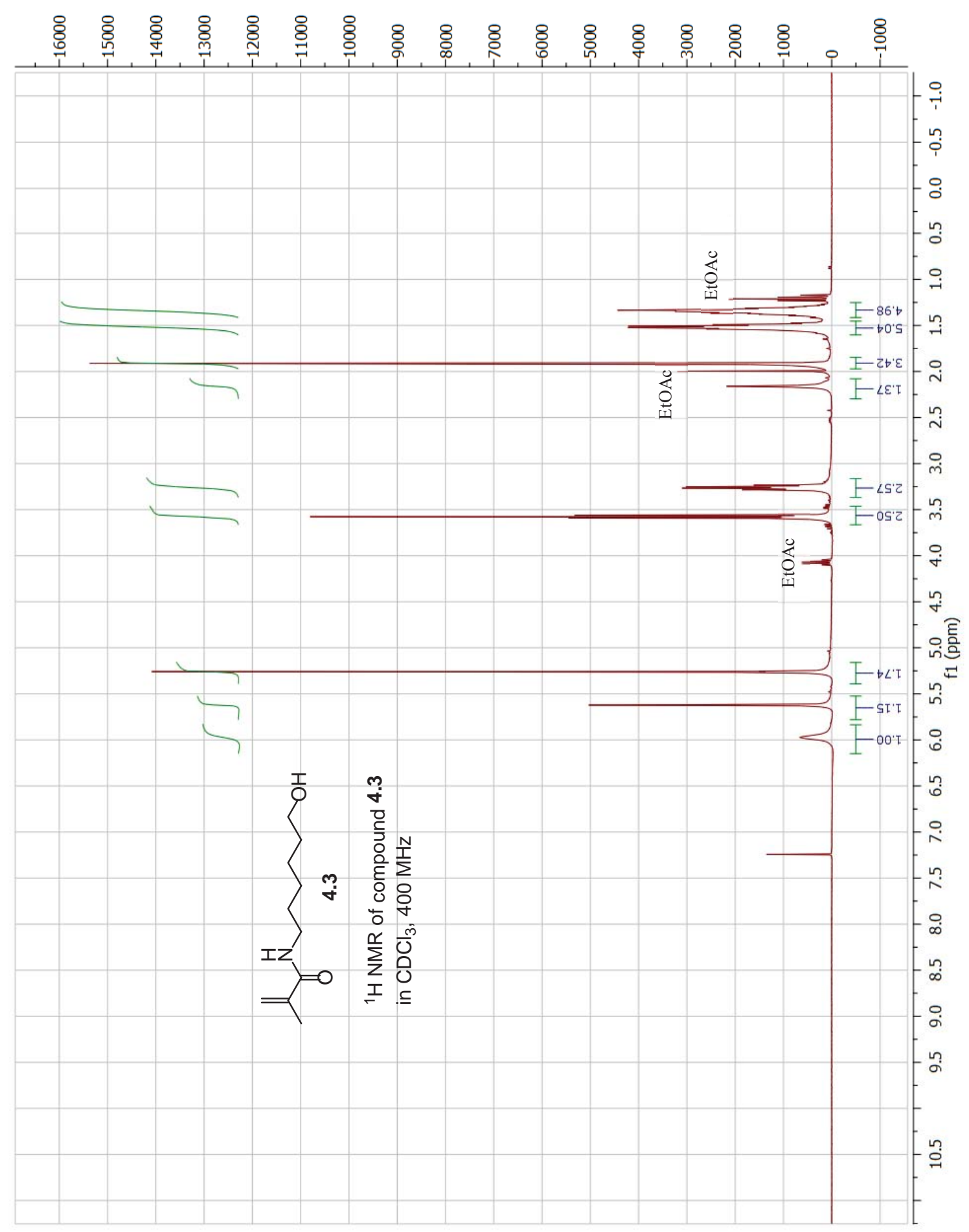

Figure B.4. ${ }^{1} \mathrm{H}-\mathrm{NMR}$ of compound 4.3 


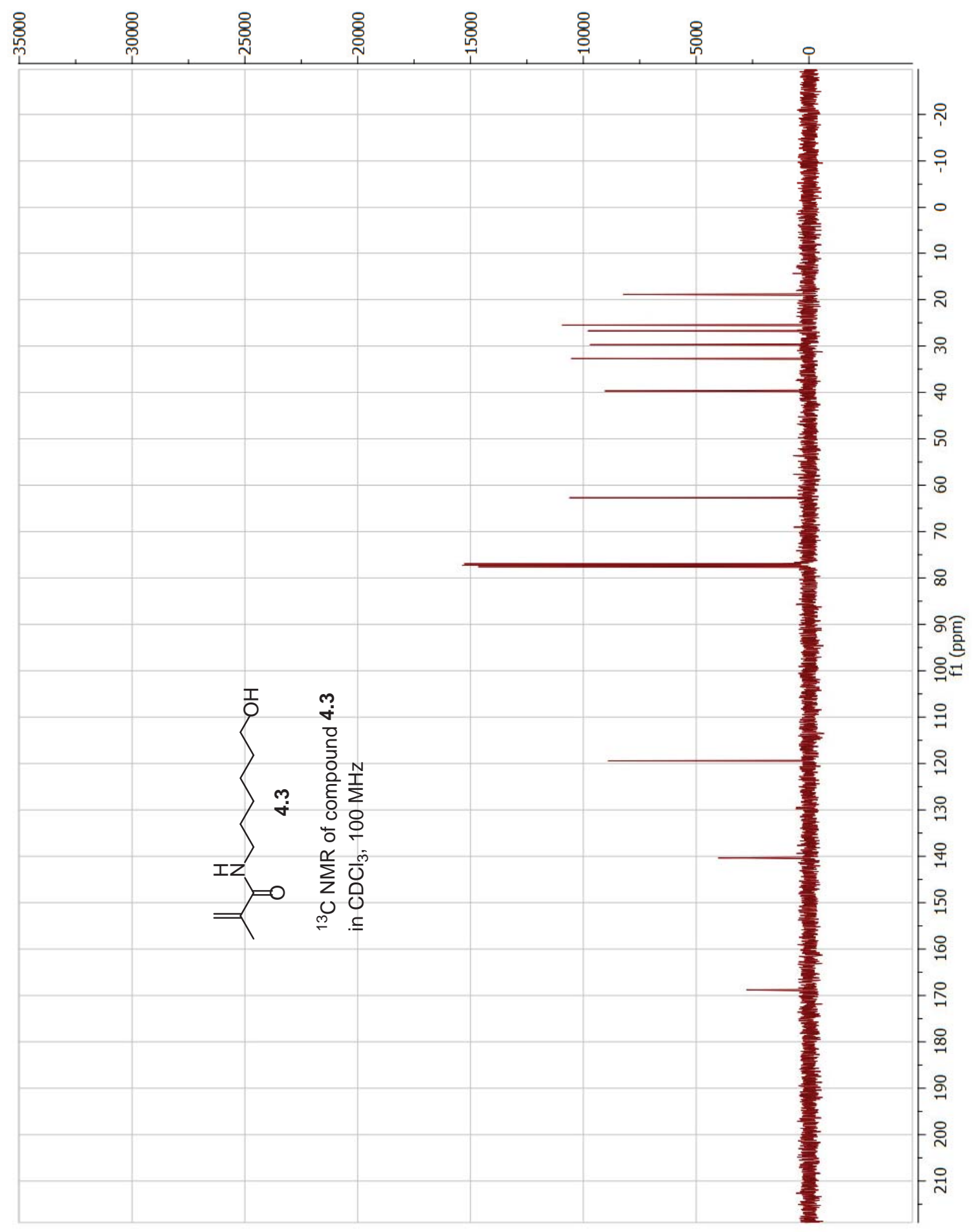

Figure B.5. ${ }^{13} \mathrm{C}-\mathrm{NMR}$ of compound 4.3 


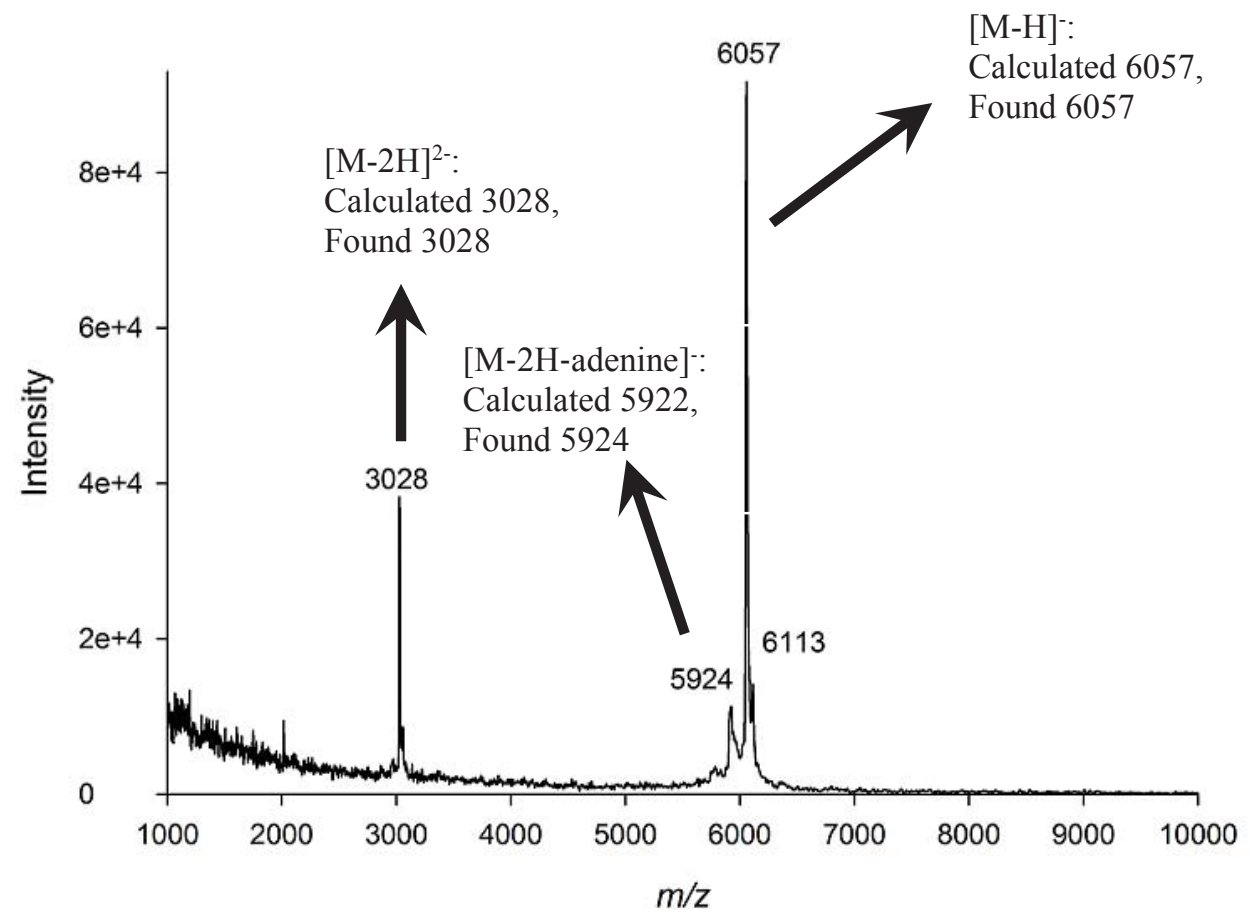

Figure B.6. MALDI-TOF mass spectrum of ODN 4.4 purified by polymerization of failure sequences 


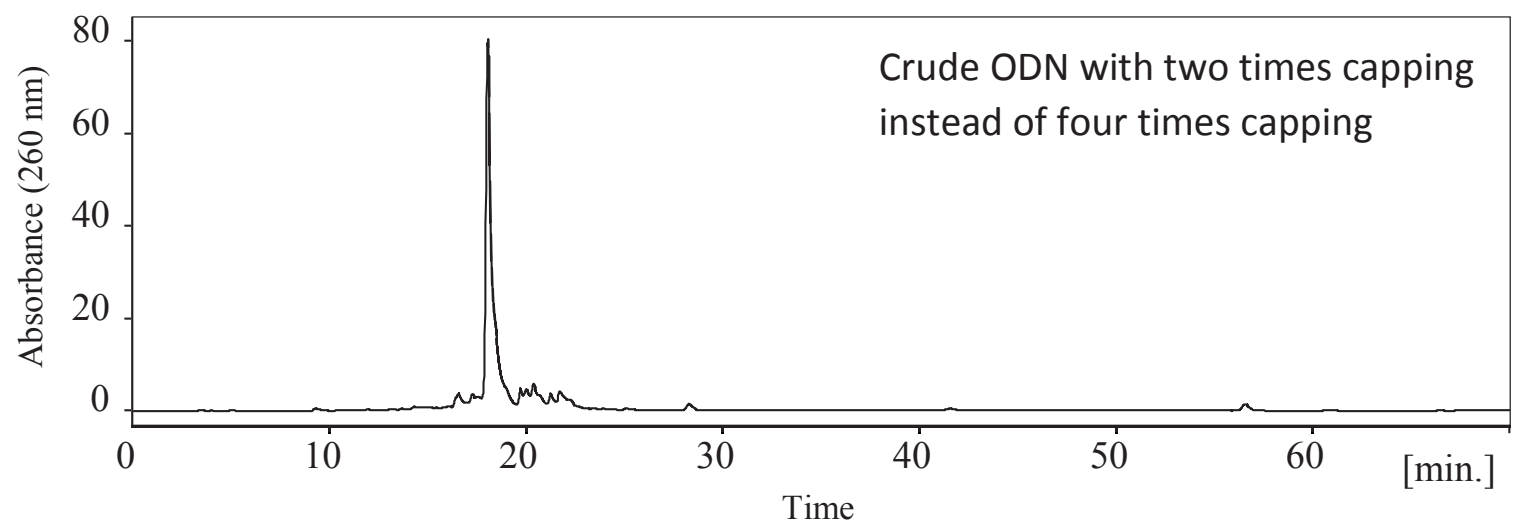

Figure B.7. HPLC profile of crude ODN with two times capping instead of four times capping 


\section{Table B.1. ODN synthetic cycle using polymerizable phosphoramidite as capping}

agent

ODN synthetic cycle using polymerizable phosphoramidite $\mathbf{4 . 1}$ as the capping agent Synthesizer: standard ABI 394 solid phase synthesizer; 4-column 8-base instrument Polymerizable capping agent: $0.2 \mathrm{M}$ solution of 4.1 in acetonitrile, placed at the bottle 5 position

The bottles for normal $\mathrm{Ac}_{2} \mathrm{O}$ capping agents are empty

Activator for the capping phosphoramidite: from the same bottle for the coupling step Synthesis scale: $0.2 \mu \mathrm{mol}$

Column used: column 2

\begin{tabular}{llll}
\hline Step number & Function number & Function name & Step time \\
\hline 1. & 106 & Begin & \\
2. & 64 & 18 To waste & 3.0 \\
3. & 42 & 18 To column & 10.0 \\
4. & Reverse flush & 8.0 \\
5. & 2 & Block flush & 4.0 \\
6. & 1 & Phos Prep & 3.0 \\
7. & 101 & Column 2 on & \\
8. & 142 & & \\
9. & 64 & Block flush & 3.0
\end{tabular}




\begin{tabular}{|c|c|c|c|}
\hline Step number & Function number & Function name & Step time \\
\hline 10. & 111 & Block vent & 2.0 \\
\hline 11. & 58 & Tet to waste & 1.7 \\
\hline 12. & 33 & $\mathrm{~B}+$ Tet to column & 2.0 \\
\hline 13. & 34 & Tet to column & 1.0 \\
\hline 14. & 33 & $\mathrm{~B}+$ Tet to column & 1.5 \\
\hline 15. & 43 & Push to column & \\
\hline 16. & 143 & Column 2 off & \\
\hline 17. & 103 & wait & 25.0 \\
\hline 18. & 64 & 18 To waste & 4.0 \\
\hline 19. & 2 & Reverse flush & 5.0 \\
\hline 20. & 1 & Block flush & 3.0 \\
\hline 21. & 41 & 15 To column & 8.0 \\
\hline 22. & 64 & 18 To waste & 4.0 \\
\hline 23. & 1 & Block flush & 3.0 \\
\hline 24. & 103 & Wait & 15.0 \\
\hline 25. & 42 & 18 To column & 10.0 \\
\hline 26. & 4 & Flush to waste & 4.0 \\
\hline 27. & 42 & 18 To column & 10.0 \\
\hline
\end{tabular}




\begin{tabular}{|c|c|c|c|}
\hline Step number & Function number & Function name & Step time \\
\hline 28. & 2 & Reverse flush & 5.0 \\
\hline 29. & 1 & Block flush & 3.0 \\
\hline 30. & 142 & Column 2 on & \\
\hline 31. & 64 & 18 To waste & 4.0 \\
\hline 32 . & 1 & Block flush & 3.0 \\
\hline 33. & 111 & Block vent & 2.0 \\
\hline 34. & 58 & Tet to waste & 1.7 \\
\hline 35. & 35 & $5+$ Tet to column & 2.5 \\
\hline 36. & 103 & Wait & 15.0 \\
\hline 37. & 34 & Tet to column & 1.0 \\
\hline 38. & 35 & $5+$ Tet to column & 1.5 \\
\hline 39. & 103 & Wait & 15.0 \\
\hline 40. & 34 & Tet to column & 1.0 \\
\hline 41. & 35 & $5+$ Tet to column & 1.5 \\
\hline 42. & 103 & Wait & 15.0 \\
\hline 43. & 34 & Tet to column & 1.0 \\
\hline 44. & 35 & $5+$ Tet to column & 1.5 \\
\hline 45. & 103 & Wait & 15.0 \\
\hline
\end{tabular}




\begin{tabular}{|c|c|c|c|}
\hline Step number & Function number & Function name & Step time \\
\hline 46. & 43 & Push to column & \\
\hline 47. & 143 & Column 2 off & \\
\hline 48. & 103 & Wait & 30.0 \\
\hline 49. & 142 & Column 2 on & \\
\hline 50. & 64 & 18 To waste & 4.0 \\
\hline 51. & 2 & Reverse flush & 5.0 \\
\hline 52. & 1 & Block flush & 3.0 \\
\hline 53. & 41 & 15 To column & 12.0 \\
\hline 54. & 103 & Wait & 22.0 \\
\hline 55. & 41 & 15 To column & 5.0 \\
\hline 56. & 103 & Wait & 15.0 \\
\hline 57. & 41 & 15 To column & 5.0 \\
\hline 58. & 64 & 18 To waste & 4.0 \\
\hline 59. & 1 & Block flush & 3.0 \\
\hline 60. & 103 & Wait & 15.0 \\
\hline 61. & 42 & 18 To column & 10.0 \\
\hline 62. & 4 & Flush to waste & 4.0 \\
\hline 63. & 42 & 18 To column & 10.0 \\
\hline
\end{tabular}




\begin{tabular}{|c|c|c|c|}
\hline Step number & Function number & Function name & Step time \\
\hline 64. & 2 & Reverse flush & 5.0 \\
\hline 65. & 1 & Block flush & 3.0 \\
\hline 66. & 105 & Start detrityl & \\
\hline 67. & 64 & 18 To waste & 4.0 \\
\hline 68. & 42 & 18 To column & 10.0 \\
\hline 69. & 2 & Reverse flush & 5.0 \\
\hline 70. & 1 & Block flush & 3.0 \\
\hline 71. & 167 & If monitoring & \\
\hline 72. & 44 & 19 To column & 25.0 \\
\hline 73. & 40 & 14 To column & 3.0 \\
\hline 74. & 135 & Monitor triyls & \\
\hline 75. & 40 & 14 To column & 25.0 \\
\hline 76. & 136 & Monitor noise & \\
\hline 77. & 40 & 14 To column & 10.0 \\
\hline 78. & 137 & Stop monitor & \\
\hline 79. & 42 & 18 To column & 10.0 \\
\hline 80. & 2 & Reverse flush & 8.0 \\
\hline 81. & 168 & If not monitoring & \\
\hline
\end{tabular}




\begin{tabular}{|c|c|c|c|}
\hline Step number & Function number & Function name & Step time \\
\hline 82. & 40 & 14 To column & 6.0 \\
\hline 83. & 3 & Trityl flush & 5.0 \\
\hline 84. & 40 & 14 To column & 6.0 \\
\hline 85. & 103 & Wait & 5.0 \\
\hline 86. & 3 & Trityl flush & 5.0 \\
\hline 87. & 40 & 14 To column & 6.0 \\
\hline 88. & 103 & Wait & 5.0 \\
\hline 89. & 3 & Trityl flush & 5.0 \\
\hline 90. & 40 & 14 To column & 6.0 \\
\hline 91. & 103 & Wait & 5.0 \\
\hline 92. & 3 & Trityl flush & 5.0 \\
\hline 93. & 42 & 18 To column & 10.0 \\
\hline 94. & 3 & Trityl flush & 8.0 \\
\hline 95. & 169 & End monitoring & \\
\hline 96. & 42 & 18 To column & 8.0 \\
\hline 97. & 2 & Reverse flush & 5.0 \\
\hline 98. & 1 & Block flush & 4.0 \\
\hline 99. & 107 & End & \\
\hline
\end{tabular}




\section{Appendix C}

Supporting Information for Chapter 5

Synthetic 5'-Phosphorylated Oligodeoxynucleotide Purification through

Catching Full-length Sequences by Polymerization 


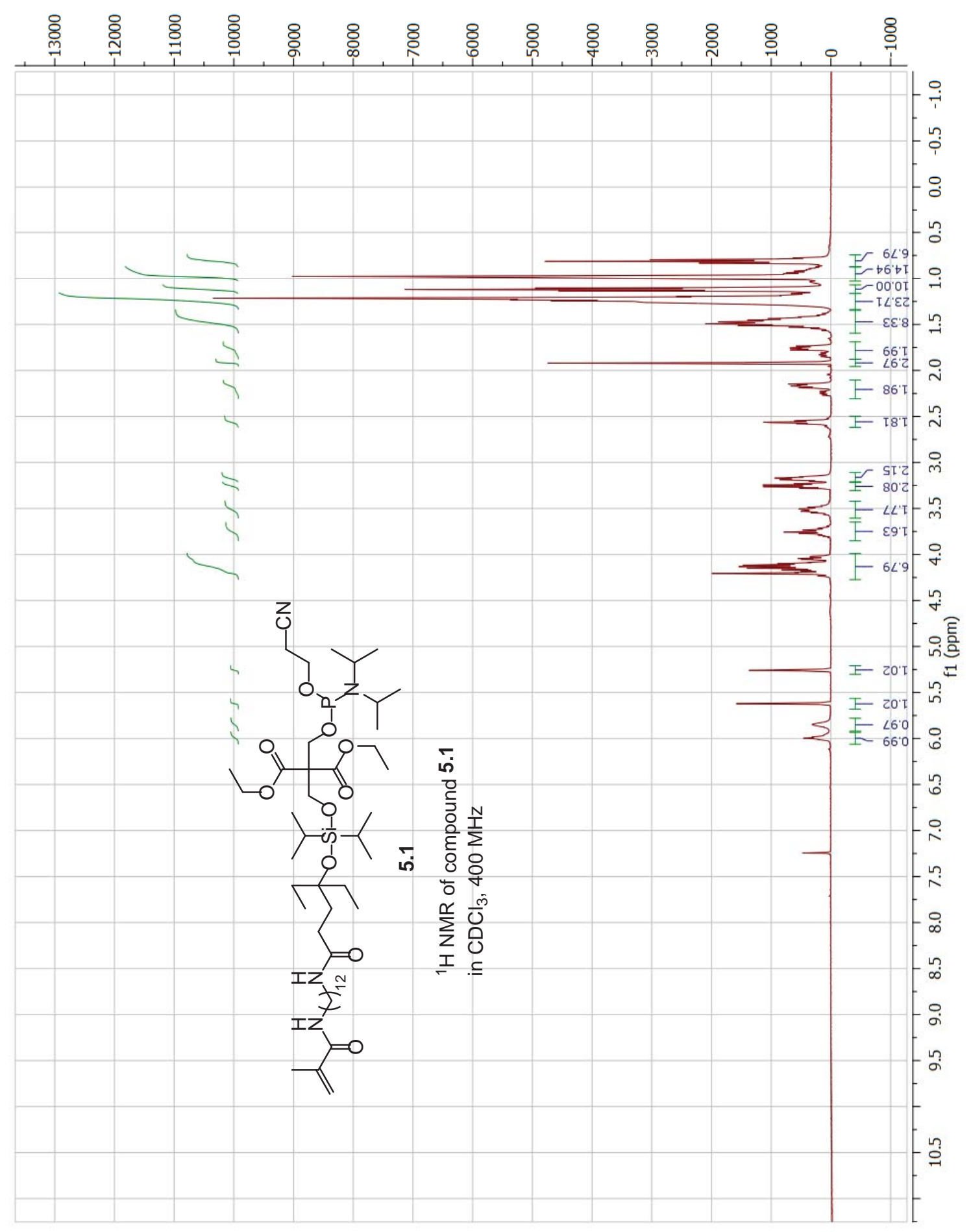

Figure C.1. ${ }^{1} \mathrm{H}-\mathrm{NMR}$ of compound $\mathbf{5 . 1}$ 


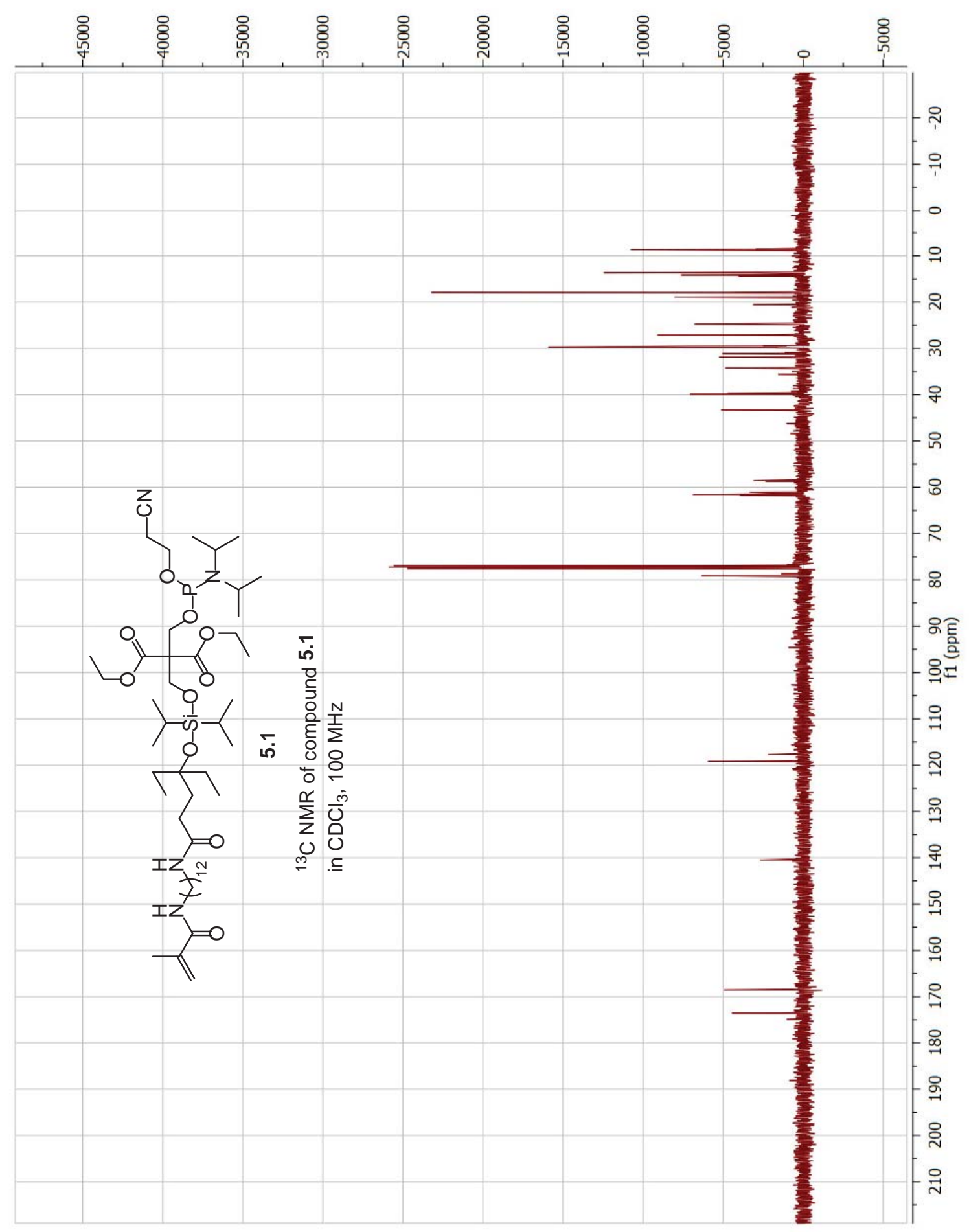

Figure C.2. ${ }^{13} \mathrm{C}-\mathrm{NMR}$ of compound $\mathbf{5 . 1}$ 


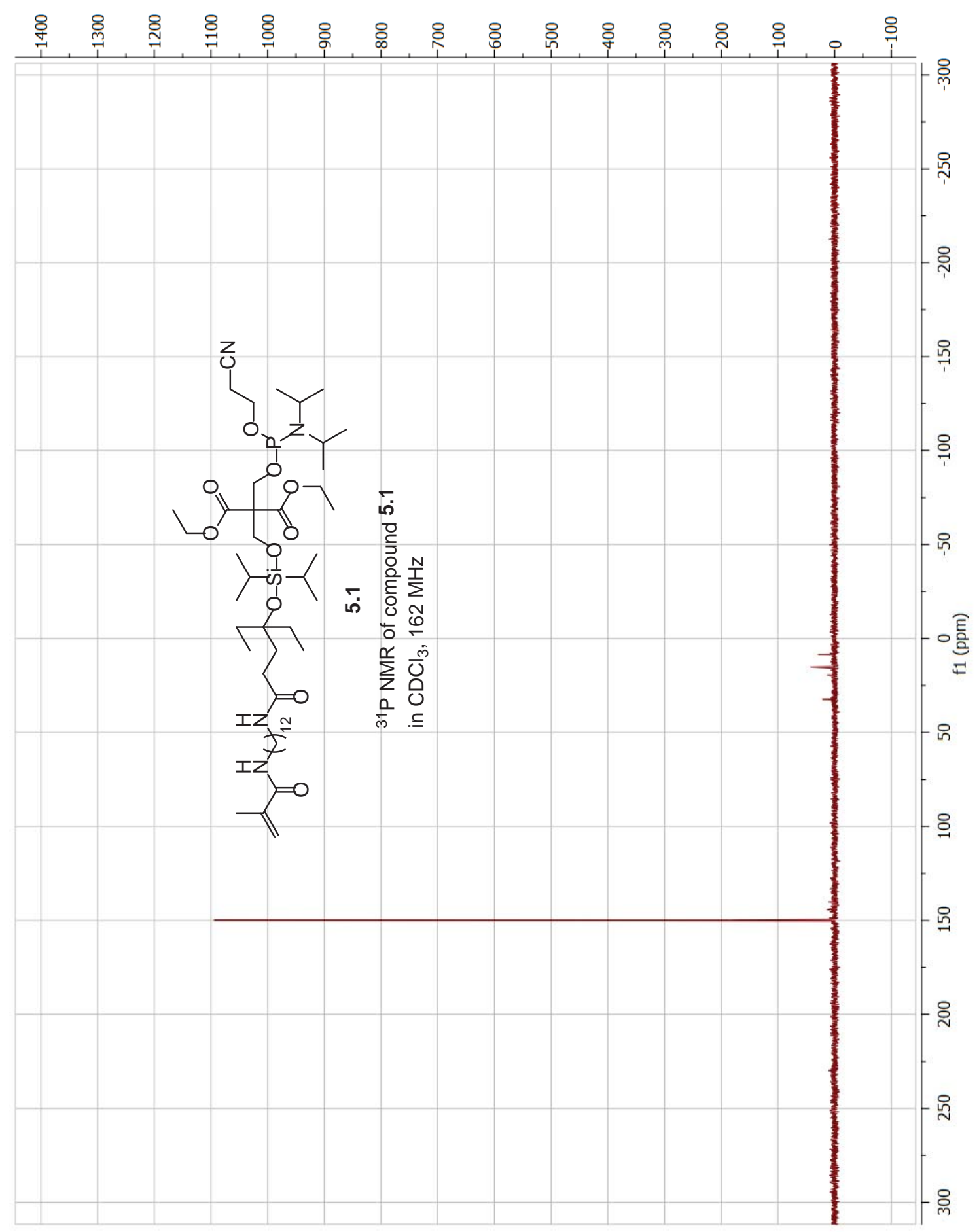

Figure C.3. ${ }^{31} \mathrm{P}-\mathrm{NMR}$ of compound $\mathbf{5 . 1}$ 


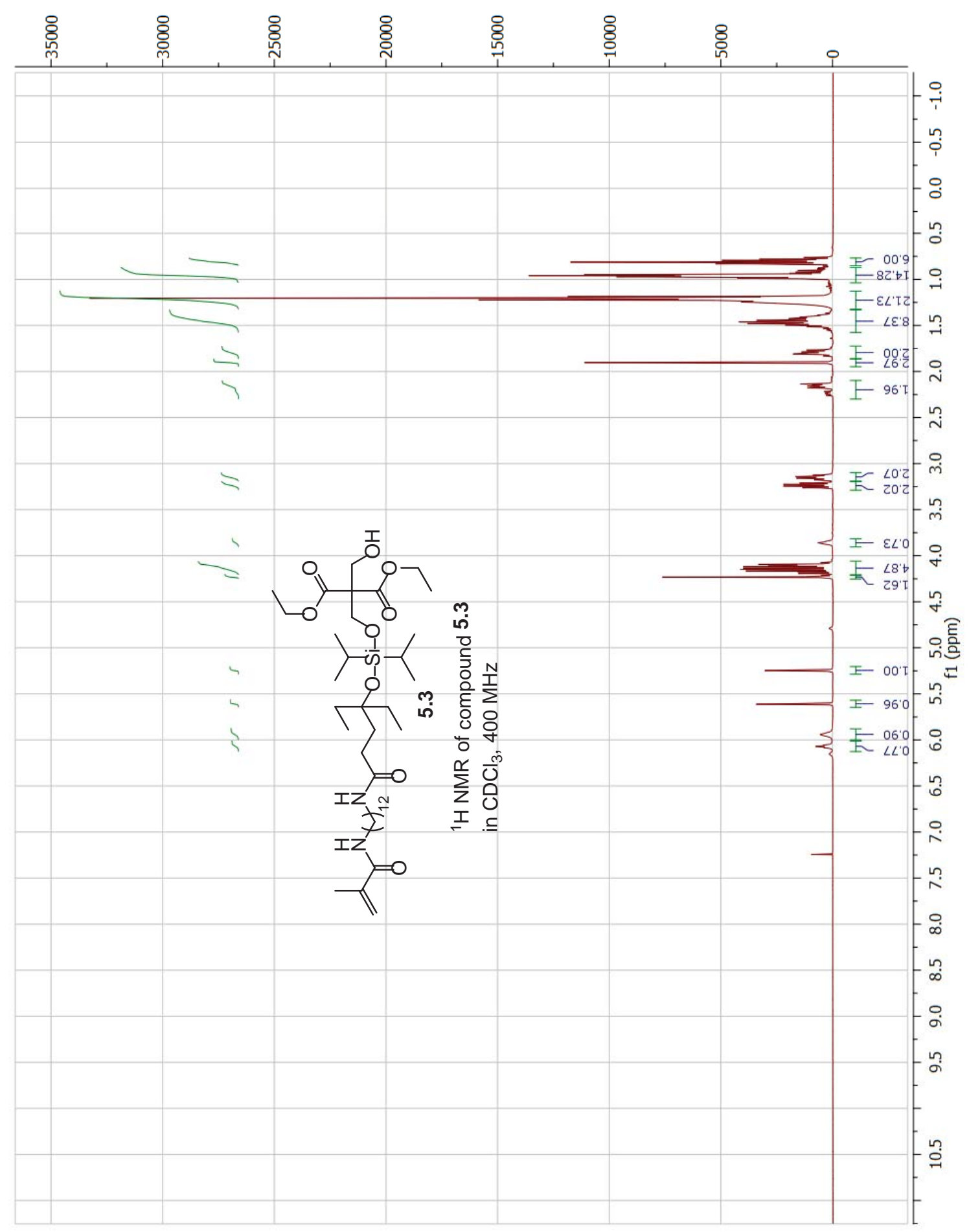

Figure C.4. ${ }^{1} \mathrm{H}-\mathrm{NMR}$ of compound $\mathbf{5 . 3}$ 


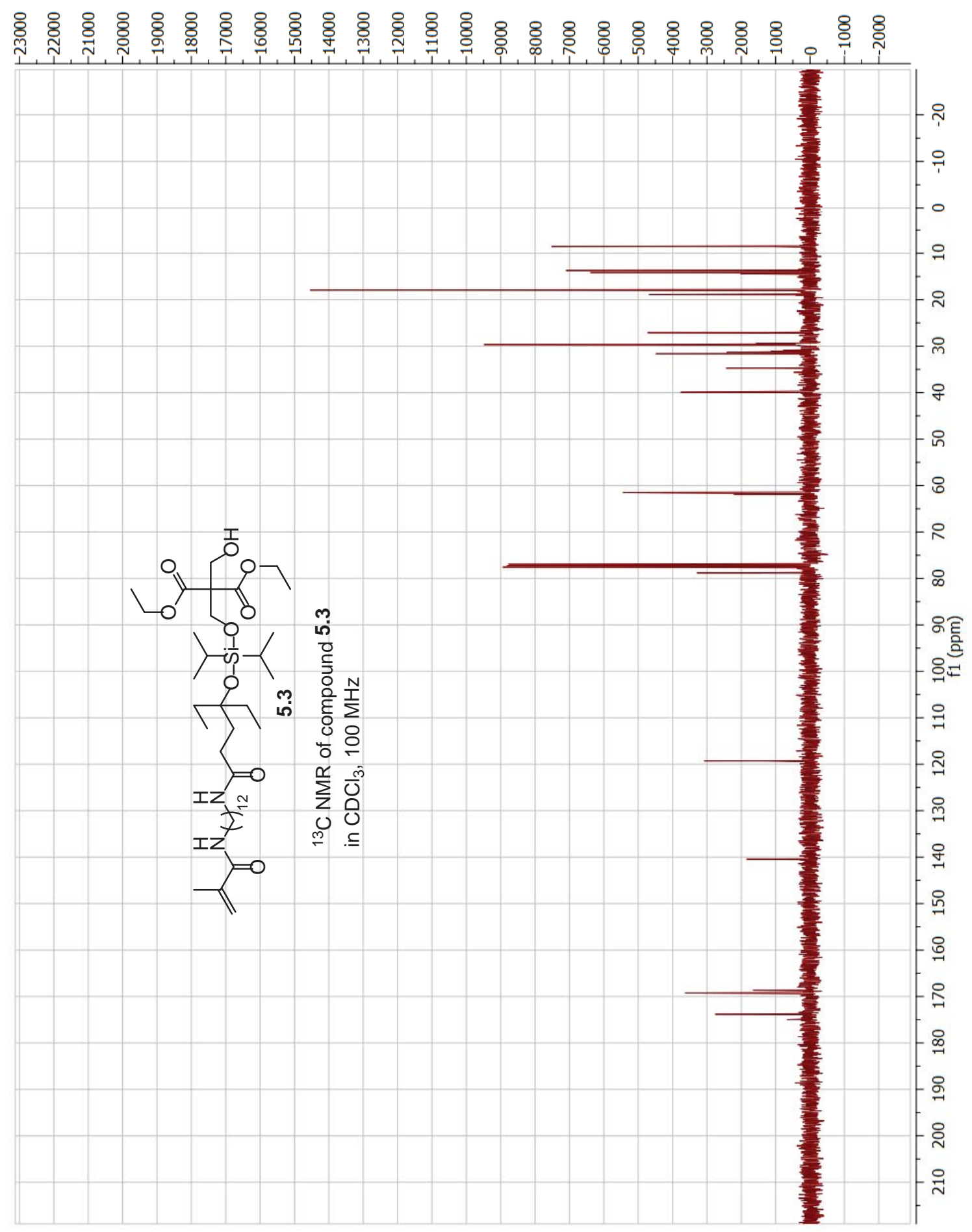

Figure C.5. ${ }^{13} \mathrm{C}-\mathrm{NMR}$ of compound $\mathbf{5 . 3}$ 


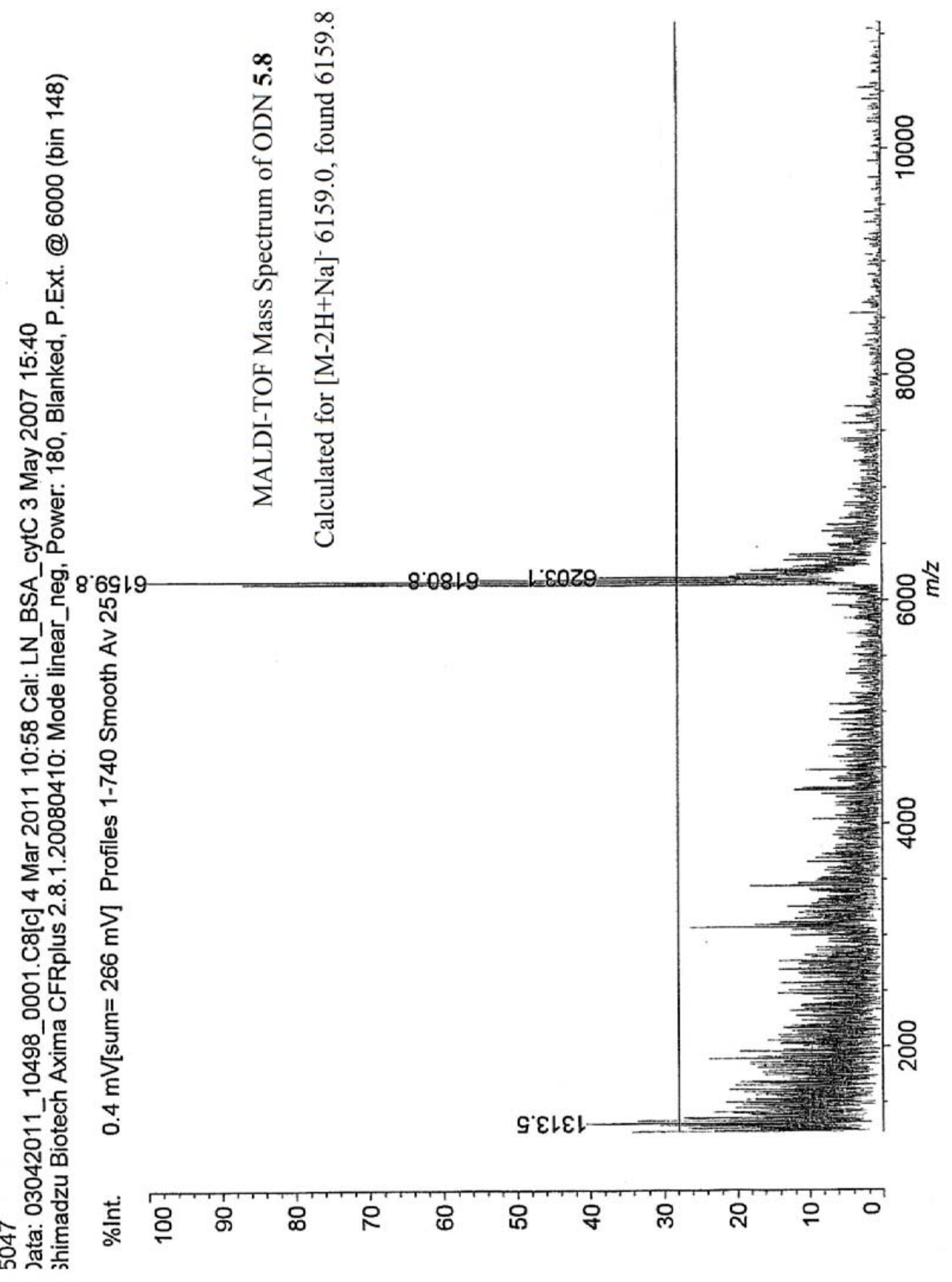

Figure C.6. MALDI-TOF mass spectrum of ODN 5.8 purified by polymerization of failure sequences 


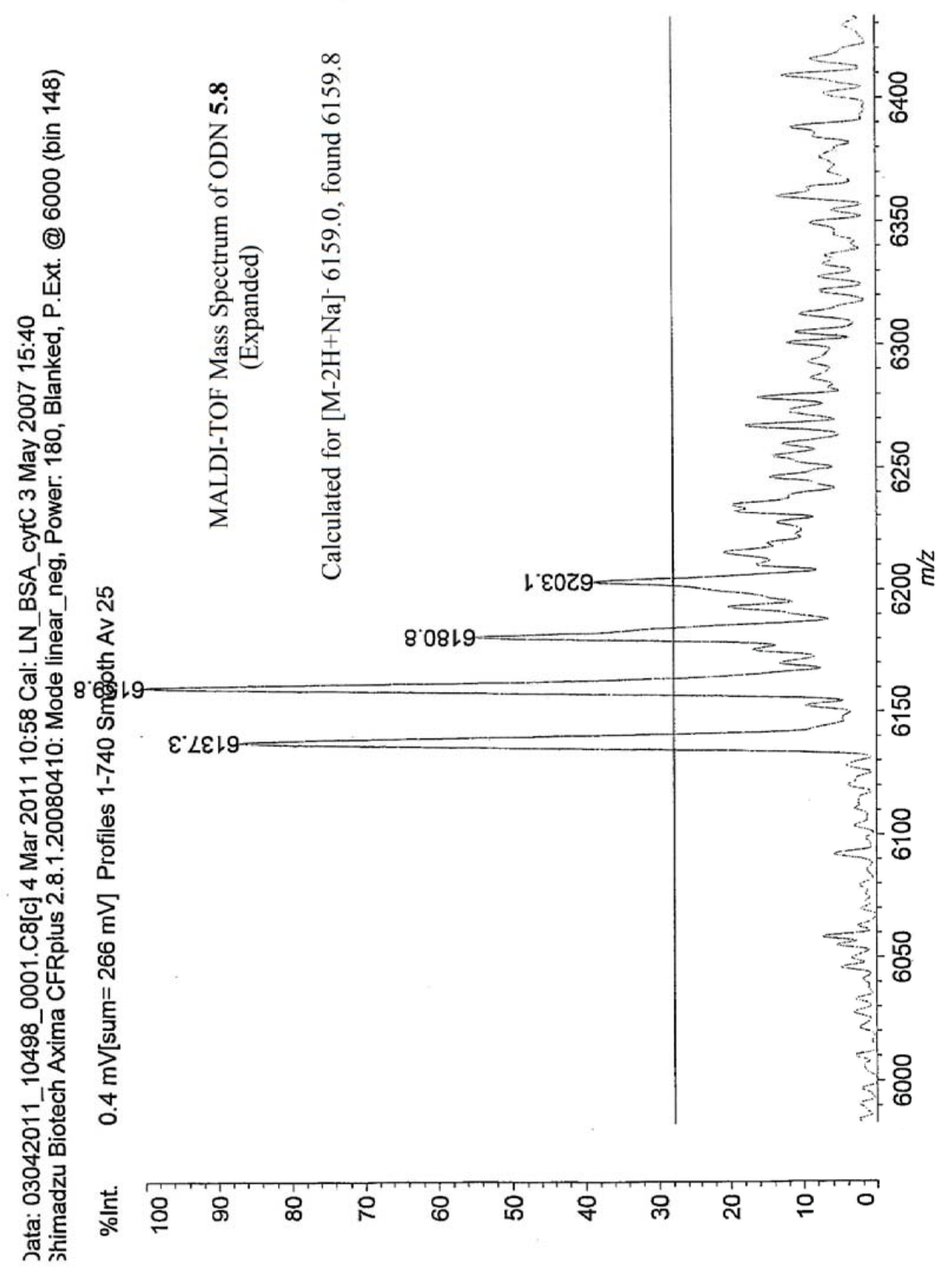

Figure C.7. MALDI-TOF mass spectrum of ODN 5.8 (Expanded) purified by polymerization of failure sequences 


\section{Appendix D}

\section{Permission from publishers}


Permission for Figure 2.1. in Chapter 2 (page 17)

$6 / 16 / 2014$

Rightslink Printable License

NATURE PUBLISHING GROUP LICENSE TERMS AND CONDITIONS

Jun 16,2014

This is a License Agreement between Suntara Fueangfung ("You") and Nature Publishing Group ("Nature Publishing Group") provided by Copyright Clearance Center ("CCC"). The license consists of your order details, the terms and conditions provided by Nature Publishing Group, and the payment terms and conditions.

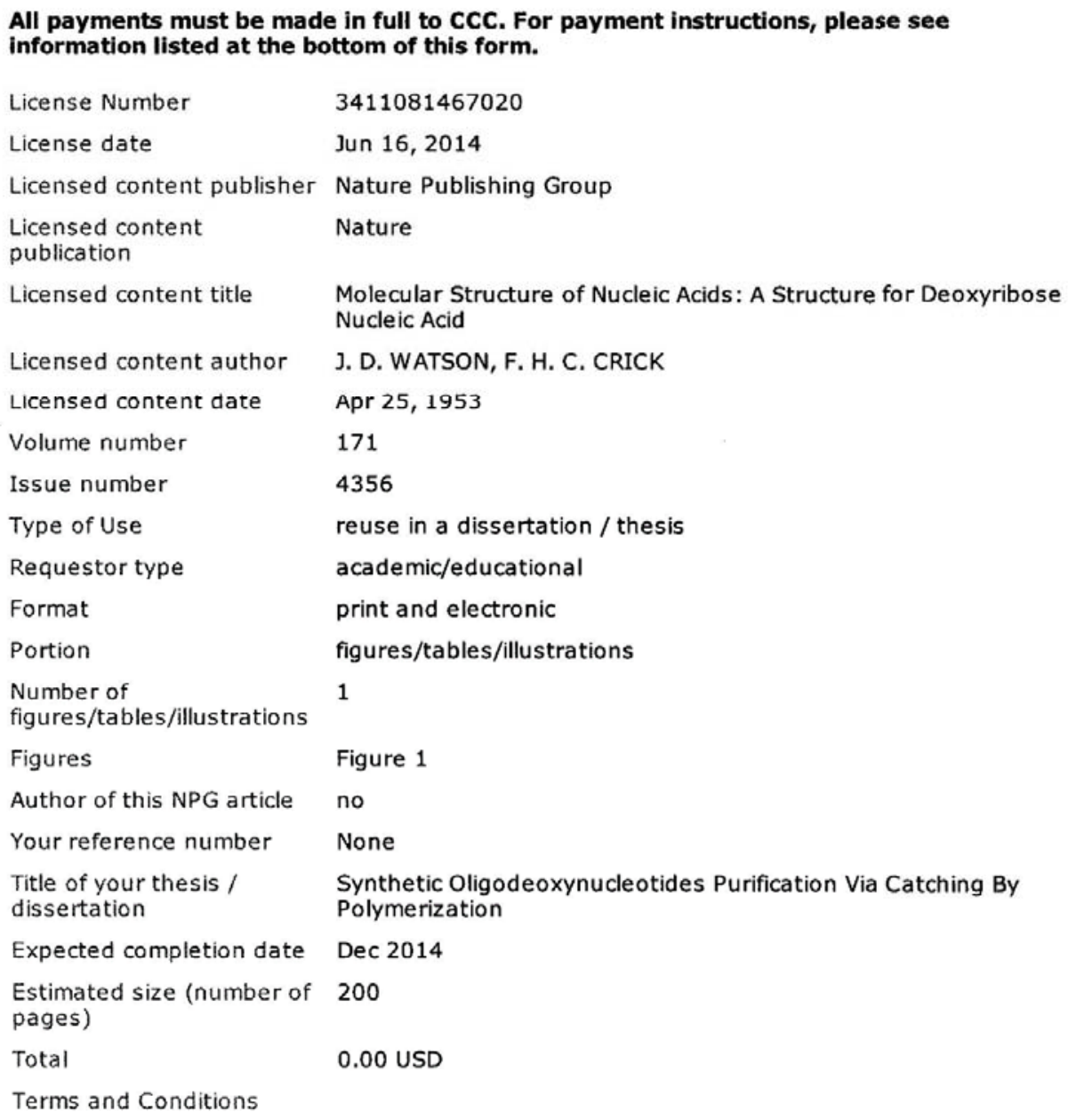

Terms and Conditions

Terms and Conditions for Permissions 


\section{Permission for Figure 2.1. in Chapter 2 (page 17)}

6/16/2014

Rightslink Printable License

Nature Publishing Group hereby grants you a non-exchsive license to reproduce this material for this purpose, and for no other use,subject to the conditions below:

1. NPG warrants that it has, to the best of its knowledge, the rights to license reuse of this material. However, you should ensure that the material you are requesting is original to Nature Publishing Group and does not carry the copyright of another entity (as credited in the published version). If the credit line on any part of the material you have requested indicates that it was reprinted or adapted by NPG with permission from another source, then you should also seek permission from that source to reuse the material.

2. Permission granted free of charge for material in print is also usually granted for any electronic version of that work, provided that the material is incidental to the work as a whole and that the electronic version is essentially equivalent to, or substitutes for, the print version. Where print permission has been granted for a fee, separate permission must be obtained for any additional, electronic re-use (unless, as in the case of a full paper, this has already been accounted for during your initial request in the calculation of a print run).NB: In all cases, web-based use of full-text articles must be authorized separately through the 'Use on a Web Site' option when requesting permission.

3. Permission granted for a first edition does not apply to second and subsequent editions and for editions in other languages (except for signatories to the STM Permissions Guidelines, or where the first edition permission was granted for free).

4. Nature Publishing Group's permission must be acknowledged next to the figure, table or abstract in print. In electronic form, this acknowledgement must be visible at the same time as the figure/table/abstract, and must be hyperlinked to the journal's homepage.

5. The credit line should read:

Reprinted by permission from Macmillan Publishers Ltd: [JOURNAL NAME] (reference citation), copyright (year of publication)

For AOP papers, the credit line should read:

Reprinted by permission from Macmillan Publishers Ltd: [JOURNAL NAME], advance online publication, day month year (doi: 10.1038/sj.[JOURNAL ACRONYM].XXXXX)

\section{Note: For republication from the British Journal of Cancer, the following credit lines} apply.

Reprinted by permission from Macmillan Publishers Ltd on behalf of Cancer Research UK: [JOURNAL NAME] (reference citation), copyright (year of publication)For AOP papers, the credit line should read:

Reprinted by permission from Macmillan Publishers Ltd on behalf of Cancer Research UK: [JOURNAL NAME], advance online publication, day month year (doi: $10.1038 / \mathrm{sj}$.

[JOURNAL ACRONYM].XXXXX)

6. Adaptations of single figures do not require NPG approval. However, the adaptation should be credited as follows:

Adapted by permission from Macmillan Publishers Ltd: [JOURNAL NAME] (reference citation), copyright (year of publication)

\section{Note: For adaptation from the British Journal of Cancer, the following credit line} applies.

Adapted by permission from Macmillan Publishers Ltd on behalf of Cancer Research UK: [JOURNAL NAME] (reference citation), copyright (year of publication)

7. Translations of 401 words up to a whole article require NPG approval. Please visit http://www. macmillanmedicalcommunications.com for more information.Translations of up to a 400 words do not require NPG approval. The translation should be credited as follows:

Translated by permission from Macmillan Publishers Ltd: [JOURNAL NAME] (reference citation), copyright (year of publication). 
Permission for Figure 2.1. in Chapter 2 (page 17)

6/16/2014

Rightslink Printable License

Note: For translation from the British Journal of Cancer, the following credit line applies.

Translated by permission from Macmillan Publishers Ltd on behalf of Cancer Research UK: [JOURNAL NAME] (reference citation), copyright (year of publication)

We are certain that all parties will benefit from this agreement and wish you the best in the use of this material. Thank you

Special Terms:

$\mathrm{vl} .1$

If you would like to pay for this license now, please remit this license along with your payment made payable to "COPYRIGHT CLEARANCE CENTER" otherwise you will be invoiced within $\mathbf{4 8}$ hours of the license date. Payment should be in the form of a check or money order referencing your account number and this invoice number 501329688.

Once you receive your invoice for this order, you may pay your invoice by credit card.

Please follow instructions provided at that time.

Make Payment To:

Copyright Clearance Center

Dept 001

P.0. Box 843006

Boston, MA 02284-3006

For suggestions or comments regarding this order, contact RightsLink Customer Support: customercare@copyright.com or +1-877-622-5543 (toll free in the US) or +1-978-6462777.

Gratis licenses (referencing $\$ 0$ in the Total field) are free. Please retain this printable license for your reference. No payment is required. 


\section{Permission for Chapter 3}

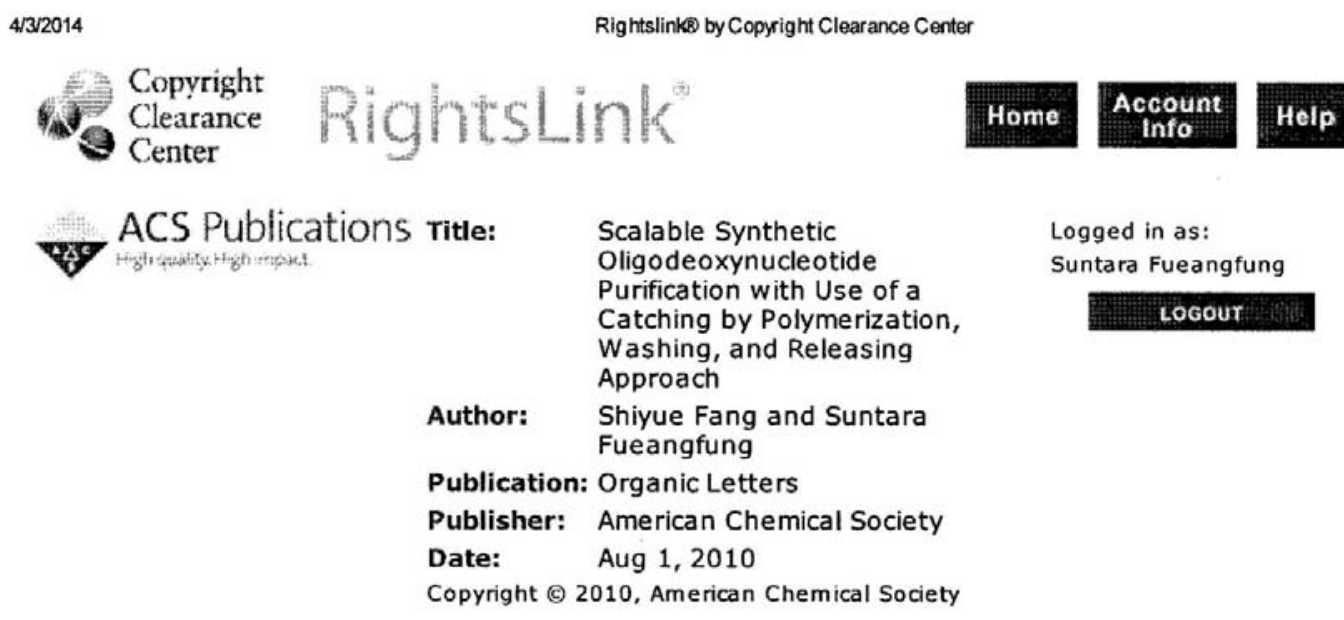

\section{PERMISSION/LICENSE IS GRANTED FOR YOUR ORDER AT NO CHARGE}

This type of permission/license, instead of the standard Terms \& Conditions, is sent to you because no fee is being charged for your order. Please note the following:

- Permission is granted for your request in both print and electronic formats, and translations.

- If figures and/or tables were requested, they may be adapted or used in part.

- Please print this page for your records and send a copy of it to your publisher/graduate school

- Appropriate credit for the requested material should be given as follows: "Reprinted (adapted) with permission from (COMPLETE REFERENCE CITATION). Copyright (YEAR) American Chemical Society." Insert appropriate information in place of the capitalized words.

- One-time permission is granted only for the use specified in your request. No additional uses are granted (such as derivative works or other editions). For any other uses, please submit a new request.

\section{BACK}

\section{CLOSE WINDOW}

Copyright (c) 2014 Copyriaht Clearance Center. Inc, All Rights Reserved. Privacy statement.

Comments? We would like to hear from you. E-mail us at customercare@copyriaht.com 


\section{Permission for Chapter $\mathbf{4}$ and Chapter 5}

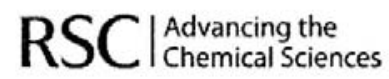

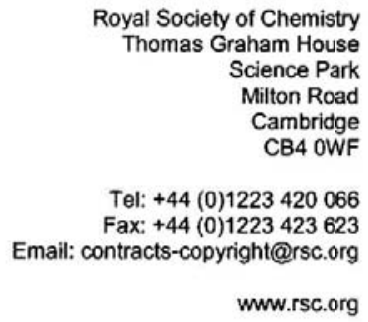

Acknowledgements to be used by RSC authors

Authors of RSC books and journal articles can reproduce material (for example a figure) from the RSC publication in a non-RSC publication, including theses, without formally requesting permission providing that the correct acknowledgement is given to the RSC publication. This permission extends to reproduction of large portions of text or the whole article or book chapter when being reproduced in a thesis.

The acknowledgement to be used depends on the RSC publication in which the material was published and the form of the acknowledgements is as follows:

- For material being reproduced from an article in New Journal of Chemistry the acknowledgement should be in the form:

2. [Original citation] - Reproduced by permission of The Royal Society of Chemistry (RSC) on behalf of the Centre National de la Recherche Scientifique (CNRS) and the RSC

- For material being reproduced from an article Photochemical \& Photobiological Sciences the acknowledgement should be in the form:

? [Original citation] - Reproduced by permission of The Royal Society of Chemistry (RSC) on behalf of the European Society for Photobiology, the European Photochemistry Association, and RSC

- For material being reproduced from an article in Physical Chemistry Chemical Physics the acknowledgement should be in the form:

- [Original citation] - Reproduced by permission of the PCCP Owner Societies

- For material reproduced from books and any other journal the acknowledgement should be in the form:

- [Original citation] - Reproduced by permission of The Royal Society of Chemistry

The acknowledgement should also include a hyperlink to the article on the RSC website.

The form of the acknowledgement is also specified in the RSC agreementlicence signed by the corresponding author.

Except in cases of republication in a thesis, this express permission does not cover the reproduction of large portions of text from the RSC publication or reproduction of the whole article or book chapter.

A publisher of a non-RSC publication can use this document as proof that permission is granted to use the material in the non-RSC publication.

VAT Registration Number: GB 342176471

Registered Charity Number: 207890 